\title{
An Ecologically Annotated Checklist of the Vascular Flora at the Chesapeake Bay Center for Field Biology, with Keys
}

\section{DANIEL HIGMAN}

Edited by Dennis Whigham, Geoffrey Parker, and Olav Oftedal

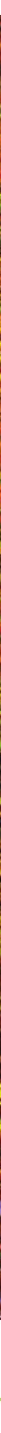



An Ecologically Annotated

Checklist of the

Vascular Flora at the

Chesapeake Bay Center

for Field Biology, with Keys

Updated Edition 



\section{An Ecologically Annotated Checklist of the}

Vascular Flora at the

Chesapeake Bay Center

for Field Biology, with Keys

DANIEL HIGMAN

Updated Edition

EDITED BY

DENNIS WHIGHAM, GEOFFREY PARKER, AND OLAV OFTEDAL

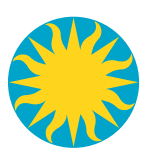

Smithsonian Environmental Research Center

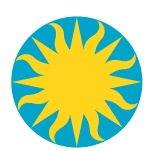

Smithsonian Institution Scholarly Press

WASHINGTON, D.C.

2016 
Originally published by the Smithsonian Institution's Office of Ecology in May I968.

Updated by the editors for the Smithsonian Environmental Research Center in July 2015.

Published by

SMITHSONIAN INSTITUTION SCHOLARLY PRESS

P.O. Box 37OI2, MRC 957

Washington, D.C. 200I3-7012

www.scholarlypress.si.edu

Cover photo: "Taking Back the Land" by A. Mark Haddon. A successional forest slowly reclaims an eighteenth-century carriage road on Smithsonian Environmental Research Center (SERC) property. The trail is now used by SERC scientists for access to research areas.

Back cover photo: Detail from aerial image taken 24 July I960 of SERC site and adjacent lands and waterways; courtesy of SERC.

Use of materials in this publication is permitted for personal, educational, or noncommercial purposes. Users must cite author and source of content and must comply with all other terms or restrictions that may be applicable. Users are responsible for securing permission from a rights holder for any other uses.

ISBN (digital): 978-I-935623-99-I

ISBN (print): 978-I-935623-98-4

Publication date (online): 6 May 2016

(2) The paper used in this publication meets the minimum requirements of the American National Standard for Permanence of Paper for Printed Library Materials Z39.48-I992. 


\section{Contents}

LIST OF FIGURES

LIST OF TABLES vii

PREFACE TO THE UPDATED EDITION $\quad$ ix

ACKNOWLEDGMENTS $\mathrm{x}$

INTRODUCTION I

Physical Environment of the Chesapeake Bay Center 3

History of Vegetation and Land Use $\quad 8$

$\begin{array}{ll}\text { Present Vegetation Types } & \text { IO }\end{array}$

STRUCTURE OF THE CHECKLIST AND KEYS 33

Nomenclature 34

Annotations $\quad 35$

Hectare Coordinates of the Chesapeake Bay Center for Field Biology $\quad 36$

Literature Cited 39

Families Represented at the Chesapeake Bay Center for Field Biology 40

Adventive and Introduced Species at the Chesapeake Bay Center 42

PLANT ANNOTATIONS (TAXONOMY AND LOCATIONAL

$\begin{array}{ll} & \\ \text { INFORMATION) } & 45\end{array}$

APPENDIX I: Additional Species Collected at the Chesapeake Bay

Center for Field Biology during the Preparation of This Checklist 225

APPENDIX II: Additional Annotations for Species in This Checklist 229

APPENDIX III: Common Names of Plants at the Chesapeake Bay

Center, Local to That Vicinity 233

APPENDIX IV: New Plant Species Found after Original Publication of Higman's Checklist $\quad 235$ 


\section{List of Figures}

I. Vicinity map

2

2. Java Farm soil map

5

3. Ivy Neck soil map

7

4. Java Farm vegetation map

I3

5. Ivy Neck vegetation map

I5

6. Java Farm hectare map

37

7. Ivy Neck hectare map

38 


\section{List of Tables}

Table I. Comparative composition of vegetation types at Java Farm and Ivy Neck

Families represented at the Chesapeake Bay Center for Field Biology

Adventive and Introduced Species at the Chesapeake Bay Center

Appendix IV. New Plant Species Found after Original Publication of Higman's Checklist 



\section{Preface to the Updated Edition}

A s part of the fiftieth anniversary celebration of the Smithsonian Environmental Research Center (SERC), it is appropriate to update and reprint the first known SERC publication. In May I968, botanist Daniel Higman published An Ecologically Annotated Checklist of the Vascular Flora at the Chesapeake Bay Center for Field Biology, with Keys through the Smithsonian's Office of Ecology. The document, henceforth referred to as The Flora, was based on Higman's exploration of plant species found on three tracts of land owned by the Smithsonian: Java Farm, Corn Island, and Ivy Neck. Plants collected during Higman's study became the foundation of the current small herbarium at SERC, and duplicate specimens were placed in the herbarium at the National Museum of Natural History's Botany Department.

Since its release, The Flora has been a valuable resource for botanists and plant ecologists working at SERC. Over the years, the number of copies-according to Higman, only Ioo copies were printed-has naturally decreased. The copies that remain in use at SERC have become worn and deteriorated. In 20I3, SERC was awarded a grant through the Smithsonian's Atherton Seidell Grant Program for the Dissemination of Previously Published Scientific Research to update and reprint The Flora and make it available online in a digital format, with a limited number of print copies distributed to libraries and repositories within and outside the Smithsonian.

The updated edition retains the taxonomic and common names as they appeared in the original document and also provides current names of species, genera, and families. Where an updated name is included, it appears in bold. Any outdated taxonomic and common names appear in parentheses after the current accepted names. We consulted the most current and reliable resources for plant taxonomy and nomenclature: Flora of Virginia Project (http://floraofvirginia.org), USDA's PLANTS Database (http://plants.usda.gov), and Kew Gardens' The Plant List (www.theplantlist.org). The USDA and Kew websites provided the current acceptable taxonomic nomenclature for species identified by Higman. To ensure consistency, a decision was made to use taxonomic names in the PLANTS Database. In keeping with the Seidell guidelines, no changes were made in the taxonomic 
keys that appear in The Flora, thus the keys may refer to prior taxonomic names that now appear in parentheses. Changes to the publication are limited to digitalizing and updating maps, a new plant species appendix, and this preface. In addition to editors Oftedal and Whigham, contributions to the appendix were provided by Jay O’Neill, John Parker, and Jessica Shue.

\section{Acknowledgments}

We especially thank Heather Soulen for moving away from her estuarine animal background to lend her skills to the task of identifying the current taxonomic nomenclature for species found in the original publication, creating GIS maps, designing the framework used to produce the revision, and creating an online searchable database using the revised edition. We thank Heather Soulen and Kristen Minogue for linking the original and revised documents to the SERC website. The efforts of Meghan Williams, working with and directing Heather in map creation using SERC's GIS facilities, is most appreciated. Finally, we thank the Smithsonian committee that provided the funding for the project through the Atherton Seidell Grant program. We are especially pleased that the funding enabled us to reproduce the first SERC publication during the year celebrating fifty years of research at the Smithsonian Environmental Research Center.

Dennis Whigham, Senior Botanist and Principal Investigator

Geoffrey G. Parker, Forest Ecologist

Olav T. Oftedal, Emeritus Scientist

Smithsonian Environmental Research Center

647 Contees Wharf Road

Edgewater, Maryland 20137 USA 
An Ecologically Annotated

Checklist of the

Vascular Flora at the

Chesapeake Bay Center

for Field Biology, with Keys

Updated Edition 



\section{Introduction}

he 690 acres of land owned by the Smithsonian Institution at the Chesapeake Bay Center for Field Biology lie on the west shore of the Bay in Anne Arundel County, Maryland, approximately 7 miles south of Annapolis and 30 miles east of Washington, D. C. The Center consists of three tracts: Java Farm, Corn Island, and the southern part of Ivy Neck peninsula (referred to herein simply as Ivy Neck). The topographic map (Figure I) shows their relationship. Java Farm and Corn Island lie on Rhode River, on opposite sides of Muddy Creek estuary. Ivy Neck lies on West River. The topography of the Center ranges from rolling upland at Java Farm to nearly level lowland at Ivy Neck. There is also much variation in the soils and drainage. The vegetation includes extensive areas of hardwood forest, abandoned fields and pastures, and coastal salt marsh. Ivy Neck also contains cultivated fields and sandy beach areas. Thus the Center presents a broad spectrum of ecological systems.

The Smithsonian Institution recently acquired the land, and in I966 established a consortium with The Johns Hopkins University and the University of Maryland for a cooperative program of ecological research. One of the first projects at the Center has been a survey of the vascular flora and the preparation of an ecologically annotated checklist with artificial keys. This checklist provides basic information required in the planning of future projects.

The floristic survey has concentrated on the areas of Java Farm and Ivy Neck, with less comprehensive coverage of Corn Island. The reason for this restriction is that the previous owner of the island is still in residence there, and much of the vegetation is subject to slight disturbance. Part of the island has been landscaped and a grass turf is maintained. The chestnut oak forest vegetation on the rest of the island resembles that of Hog Island, which is part of Java Farm.

As background information the physical environment, history of land use, and present vegetation at the Center are presented in the first part of this introduction. The second part will explain the checklist, the format of its keys, and the ecological annotations which accompany each entry. 
Figure 1.

\section{VICINITY OF THE SMITHSONIAN ENVIRONMENTAL RESEARCH CENTER}

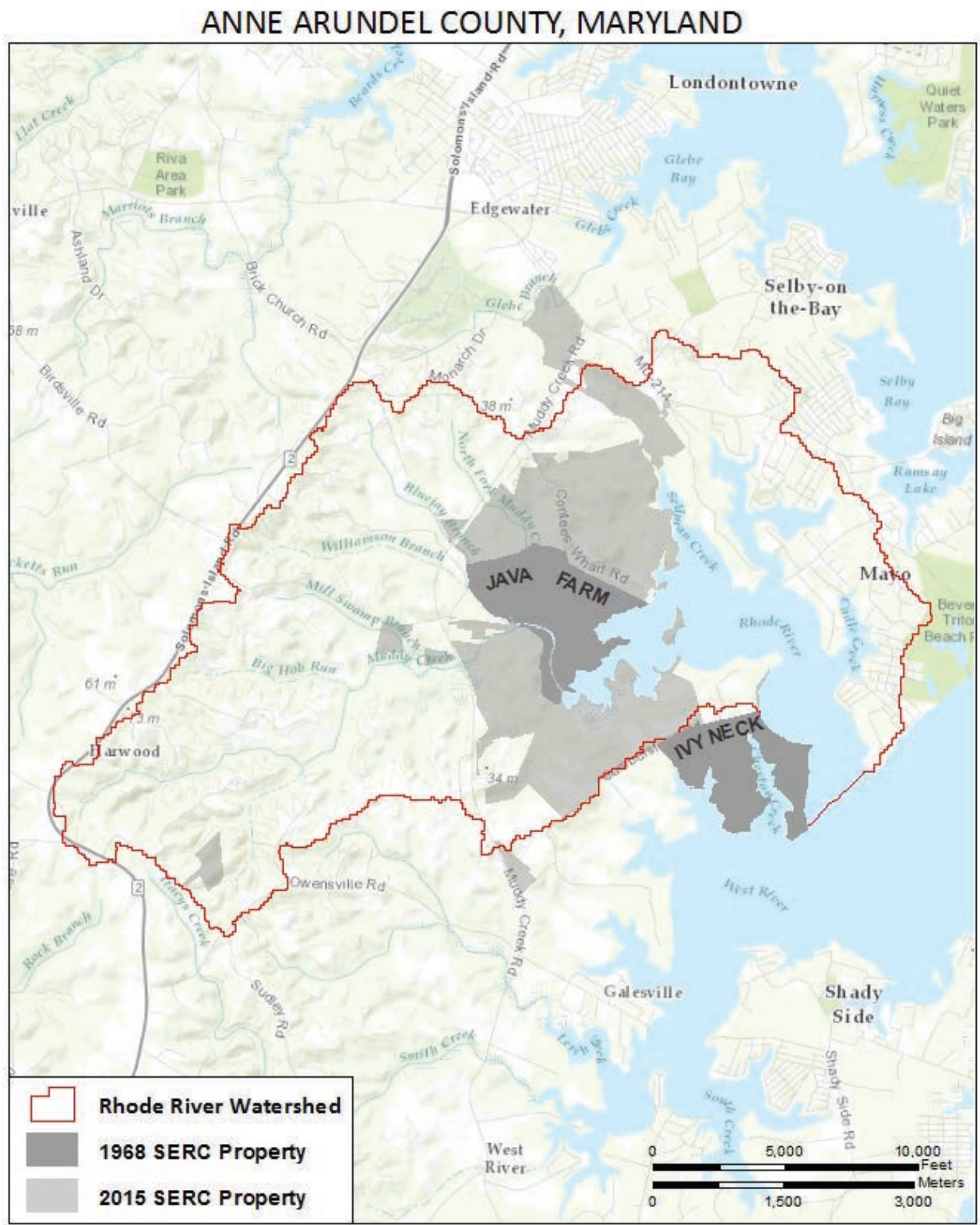




\section{Physical Environment of the Chesapeake Bay Center}

The physiography of the Center ranges from rolling upland at Java Farm to gently south-sloping lowland at Ivy Neck. Java Farm has two steep north-south ridges near the headquarters area, of which the taller has an elevation of too feet. Elsewhere, the elevation averages 20 to 60 feet. Moderately steep forested slopes occur in all directions. The Farm lies at the mouth of the watershed of Muddy Creek, and is drained by the north fork of this creek and by several springs. Although most of the farm is well drained, one abandoned pasture is vernally inundated until May or June.

Muddy Creek becomes tidal near the junction of its forks and apparently grows increasingly saline toward its estuary, as reflected by a gradient in the vegetation. Salt marshes line much of the coast of Java Farm. One marsh at the mouth of Fox Creek drainage (near the head of Fox Creek estuary) changes abruptly from freshwater to salt.

In contrast to Java Farm, Ivy Neck is virtually level and poorly drained. Three small streams drain the northern part, but on the peninsulas much of the soil is vernally saturated and the many small coves are closed by salt marshes. Severe erosion occurs along the coast, especially at the confluence of Rhode and West Rivers.

The soils of the Chesapeake Bay Center range in texture from loamy sand to silt loam. Fine sandy loams predominate at Java Farm, silt loams at Ivy Neck. This distribution of soil types reinforces the differences in drainage. The soil maps (Figures 2 and 3) illustrate the distribution of 15 soil types, according to a soil survey of Anne Arundel County now being conducted by the U. S. Soil Conservation Service. Detailed descriptions of the compositions of these types will not become available until the soil survey is published in 1969. However, three of the types were described in an earlier soil survey (Phillips, Perkins, and Winant, I928). Brief descriptions of these three soil types are given below:

I. Collington Fine Sandy Loam: Brown surface loam and underlying glauconite, both with a high iron content, friable but sticky. Substratum of fine sand, silt, and clay. Drainage excellent. Agriculturally important. Needs moderate amounts of lime. Much of the area formerly mapped under this type is now designated Monmouth Fine Sandy Loam.

2. Keyport Silt Loam: Brown surface loam with a compact underlying hardpan one or two feet deep. Drainage poor. Deficient in phosphate and lime.

3. Elkton Silt Loam: Similar to Keyport Silt Loam. Drainage and agricultural value poor. Much of the area now mapped under this type was formerly designated Keyport Silt Loam.

Coastal plain sediments underlie virtually all of Anne Arundel County. At the Chesapeake Bay Center they are approximately 2,000 feet thick. The most recent deposits are of Pleistocene age. These cover much of Ivy Neck and correspond approximately with the occurrence of Keyport silt loam. On Java Farm the Pleistocene deposits have been eroded away to reveal those of Miocene and Eocene age. These older sediments are composed of sand, gravel, marl, silt, and clay. Some 
Figure 2. (Opposite)

\section{SOIL TYPES AT JAVA FARM AND CORN ISLAND}

\section{Loam}

762 Donlonton

\section{Sandy Loam}

334 Adelphia

774 Colemantown

\section{Fine Sandy Loam}

323 Collington

373 Monmouth

763 Donlonton

\section{Silt Loam}

318 Marr

341 Shrewsbury

581 Bibb

771 Colemantown

\section{Tidal Marsh}

4

\section{Man Made}

9 


\section{SOIL TYPES AT JAVA FARM AND CORN ISLAND}

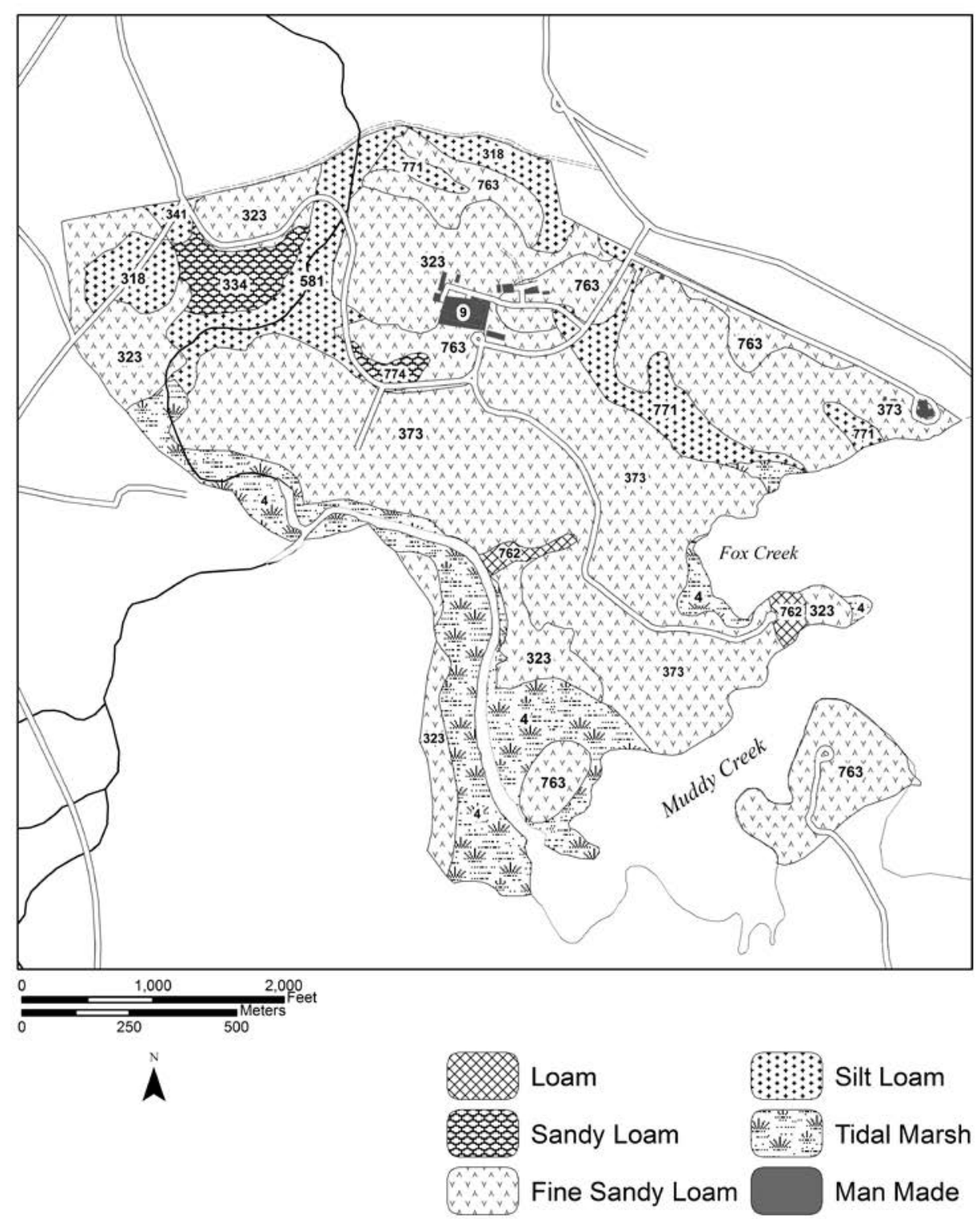


Figure 3. (Opposite)

\section{SOIL TYPES AT IVY NECK}

\section{Loamy Sand}

377 Monmouth

\section{Sandy Loam}

334 Adelphia

\section{Fine Sandy Loam}

373 Monmouth

\section{Silt Loam}

401 Keyport

411 Elkton

771 Colemantown

\section{Tidal Marsh}

4

\section{Man Made}

9 


\section{SOIL TYPES AT IVY NECK}

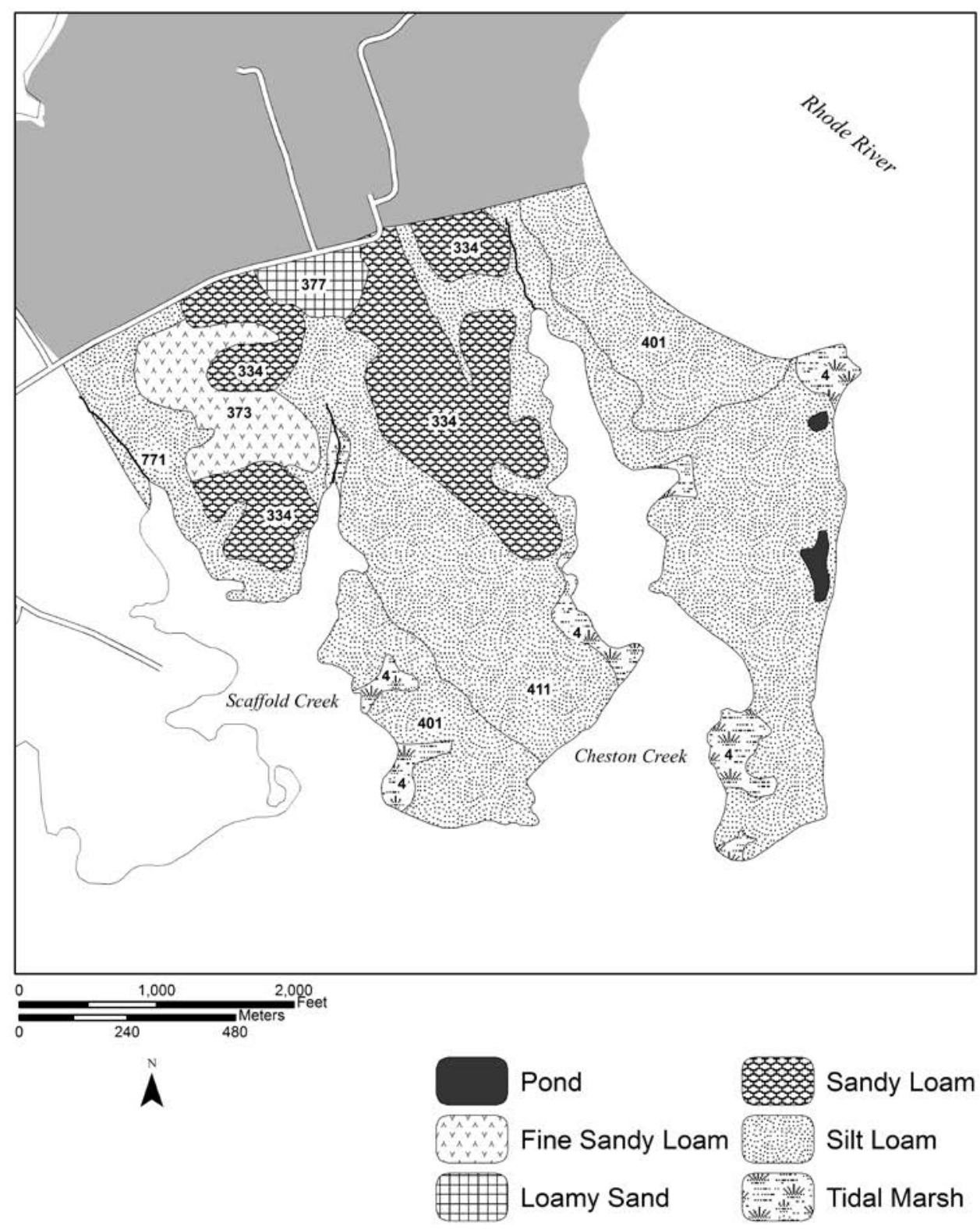


contain artesian aquifers. The deeper strata go down to Lower Cretaceous age, and finally reach a crystalline basement rock of undetermined depth.

The climate is characterized by mild summers and winters, moderated by the proximity of Chesapeake Bay. Approximate annual temperatures range from I. $6^{\circ} \mathrm{C}\left(35^{\circ} \mathrm{F}\right)$ in January to $25.0^{\circ} \mathrm{C}\left(77^{\circ} \mathrm{F}\right)$ in July. The growing season is about 203 days, from mid-April to late October. Annual precipitation averages 44 inches, usually reaching a maximum in August, but may be quite variable for single months. The annual snowfall averages 2I inches (Mack, ig62).

\section{History of Vegetation and Land Use}

The presettlement forest of Anne Arundel County probably consisted of mixed mesophytic hardwoods, with pine on the drier sites. In an attempt to reconstruct this forest, Braun (I950) places the boundary of her Oak-Chestnut and OakPine forest regions approximately within the county. On the Maryland Coastal Plain these two regions correlate roughly with the Wicomico and Talbot Pleistocene terraces, both of which occur at the Chesapeake Bay Center. The canopy of Braun' s Oak-Chestnut forest includes American chestnut, beech, white oak, Spanish oak, red maple, and pignut hickory. The Oak-Pine forest differs from this primarily in having much more sweet gum, less chestnut, and persistent stands of loblolly and Virginia pines on the drier sites. On the upland the OakPine forest also contains black, scarlet, and post oaks; on the lowland are loblolly pine, sweet gum, sour gum, red maple, pin and willow oaks, beech, and tuliptree. Shelford (I963) describes a similar canopy composition for the area, but adds shagbark and mockernut hickories and blackjack oak. A historical reference to Ivy Neck (Kelly, I965) mentions a stand of tuliptree on a hilltop near the Center and one of white oak on the adjacent lowland during the I650's. Virtually all of the above species except shagbark hickory now occur at the Center (see next section).

The composition of the presettlement forest was subject to several influences, but at present it is difficult to draw conclusions as to their relative importance. Shelford (I963) describes the inhibitive effect of the selective consumption of nuts and berries by deer, bears, squirrels, etc., and of the selective browsing of tree seedlings by the deer. However, Hairston, Smith, and Slobodkin (I960) point out that in order for a community to persist the herbivores must be limited by predation below the level at which they deplete the vegetation, or the depleted species will be replaced by more resistant ones. Major predators in the presettlement forest included bears, cougars, wolves, bobcats, and foxes (Shelford, I963). The last two still persist in the vicinity of the Center.

Storms and hurricanes, aided by the county's normally heavy precipitation, probably caused periodic damage. Craven (1926) notes five major storms between the years 1658 and I787; other big storms have been recorded at io- to 20 -year intervals in the late igth Century. 
Probably the most important disturbances to the presettlement forest were caused by Indians. Indian occupation at the Chesapeake Bay Center is documented by 22 heaps of discarded oyster shells, II of which are large enough to indicate permanent campsites. Fragments of pottery permit five of these sites to be dated within the interval 250 B.C. to I200 A.D. Other artifacts indicate that besides oysters the Indians ate deer, gathered nuts and other fruits, and probably cultivated corn. They killed the small game and cut the young trees for firewood, moving on when these resources were temporarily exhausted (H. T. Wright, unpublished data). By I608, however, these sedentary Indians had disappeared, apparently driven away by the warlike Susquehannocks, and the western shore of the Bay north of the Patuxent River was uninhabited (Marye, I955).

Marye describes the Susquehannock practice of setting recurrent forest fires, principally ground fires, to encourage the growth of grass for deer and elk. Early settlers held these fires partly responsible for huge areas of "barrens," or "sapling land," on the Maryland Piedmont, and for an extremely thin shrub layer in the forest of the Coastal Plain. The Indians also set fires to drive game and to clear village sites (Middleton, I953). Early settlers imitated the practice of forest burning to benefit their cattle and to clear fields (Marye, I955).

However, the importance of forest fires should not be overestimated. Fire did not prevent the thin-barked tuliptree or yellow-poplar from being a characteristic species, as evidenced by the names Tulip Hill (Kelly, I965), Poplar Neck, Poplar Knoll, etc. given by early settlers. The nuts of other fire-sensitive trees, such as beech and hickory, were eaten by the Indians who left the shell heaps and probably also by the later Susquehannocks. The normally heavy rainfall and numerous streams in Anne Arundel County probably kept the forest fairly moist and limited the spread of ground fires.

Colonial settlement in the county began about the year 1650 , and tobacco soon became the principal crop. It was raised throughout the vicinity of the Chesapeake Bay Center (Kelly, I965). As tobacco consumes much nitrogen and potash, only freshly cleared land produced good crops, and the forest was rapidly cleared to yield the best crops obtainable (Craven, I926). Soil erosion and stream siltation became so serious that in I704 and I743 laws were passed against the clearing of stream banks (Middleton, I953). For this reason, and because of rough terrain or inaccessibility, part of the present forest on Java Farm (along Muddy Creek and on Fox Point and Hog Island) may have escaped from cultivation, although it was almost certainly lumbered, burned, and browsed by cattle. This forest has not been cultivated at least since 1846 .

Colonial cultivation methods encouraged erosion and the development of hardpan. After a few tobacco crops the land was briefly farmed to corn and wheat, then abandoned. Tobacco exports periodically flooded the British market, producing severe depressions and the abandonment of fields (Craven, i926). Reaccumulation of soil nutrients probably was slow. 
Before I800 no fertilization or renewal of the soil was attempted. The monopoly of arable land for tobacco deprived livestock of corn and pasturage. Cattle browsed the forests and probably grazed the salt marshes. There is no available record of the marshes having been cultivated, mowed, or reclaimed.

Between I800 and I850 the fertilization of fields with manure, marl, and gypsum developed and slowly became standard practice. Soil erosion was checked. Large tobacco plantations gave way to small farms where more efficient use of fertilizer was possible and crops were diversified. General prosperity continued until the Civil War (Craven, I926). A postwar depression crippled agriculture until I880, after which grain and truck farming slowly recovered.

Java Farm served as a dairy from I9I5 until its abandonment in I945. Although the forest there was not lumbered during this period, fallen logs were removed every spring. Cultivated fields were rotated between corn and barley, but from I937 to I945 alfalfa was largely substituted for corn. The present meadow north of the headquarters area was used as pasture. No cultivation, burning, or mowing of the marshes took place. Since the dairy was abandoned in I945, it has remained unused. No fires or tenant farming disturbed the development of the vegetation. A broad spectrum of communities now occurs.

Portions of Ivy Neck are still under cultivation to corn and wheat. One field was abandoned in I963, but was recultivated in I967. Both Scaffold Peninsula (between Scaffold and Cheston Creeks) and Cheston Peninsula (between Cheston Creek and Rhode River) were entirely cultivated until about I9oo. The present deciduous forest on Scaffold Peninsula has apparently developed naturally since the abandonment of agriculture there. It was lumbered about I942. The loblolly pine stand on Cheston Peninsula was planted in I933 and has been left undisturbed.

\section{Present Vegetation Types}

The present vegetation of the Chesapeake Bay Center falls into six major categories: hardwood forest, coniferous forest, cultivated field, formerly cultivated field, freshwater marsh, and salt marsh. Within each category variations occur in the composition of the vegetation. These variations usually take the form of patterns of plant communities, referred to here as vegetation types. The boundaries of the types are not always sharply distinctive, especially where the vegetation is immature, but they form a mosaic within the hardwood forest and other main categories.

The present vegetation types provide baselines for studies of vegetation development. The similarities and differences among the types in each major category may reflect environmental characteristics or recent histories, but further research is required to establish correlations. Most of the Center has been abandoned from cultivation within the past 70 years or less, and the vegetation in these areas is still immature and changing considerably. The older hardwood forests appear to be mature and relatively stable. 
The two vegetation maps of the Center (Figures 4 and 5) are based on 28 vegetation types, which are listed in the legends of the maps. Details of the types are given in Table I, which includes lists of the more abundant plants and provides a basis for comparing the vegetation on Java Farm and Ivy Neck. There is less overlap between the vegetation types of the two areas than might be expected, probably in part because of the more level topography and poorer drainage at Ivy Neck, and in part because of differences in recent historical land use. No vegetation map of Corn Island has been prepared.

The remainder of this report will explain the structure of the checklist and of its artificial keys. The ecological annotations which show the relationship of each species to its vegetation pattern will also be described. 
Figure 4. (Opposite)

\section{VEGETATION TYPES AT JAVA FARM}

\section{FOREST TYPES}

Hardwoods

1A. Beech, Tuliptree

1B. Beech, Tuliptree, Oaks, Hickories

1C. Beech, Tuliptree, White Oak, Sycamore, Sweetgum

2. Tuliptree, Black Oak, Blackgum

3. White, Black, and Spanish Oaks, Sweetgum

*4A. Chestnut Oak, White and Black Oaks, Hickories

(4B to AD only at lvy Neck)

5. Spanish and Willow Oaks, Sweetgum, Tuliptree

6A. Walnut, Sweetgum

6B. Walnut, Sweetgum, Spanish Oak, Beech

7A. Locust, Sweetgum, Elm

7B. Locust, Black Oak, Sweetgum, Red Maple, Sassafras

7C. Locust, Sweetgum, Tuliptree, Sycamore

(7D to 7F only at lvy Neck)

8A. White Ash, Sycamore

8B. White Ash, Sycamore, Red Maple, Elm, Sweetgum

9. Tuliptree, Sweetgum, Red Maple, Sassafras

10. Dogwood, Sweetgum, Red Maple, Sassafras

(11 to 17 only at Ivy Neck)

Conifers

18A. Virginia Pine predominant

FOREST TYPES (continued)

Conifers (continued)

18B. Virginia Pine, Sweetgum, Tuliptree, Hickories

*19A. Loblolly Pine, Sweetgum (pine predominant)

(19B and 19C only at lvy Neck)

\section{ABANDONED FIELD TYPES}

(20 and 21 are CULTIVATED FIELD

TYPES, and occur only at lvy Neck)

22A. Reed Canary Grass

22B. Kentucky Bluegrass

*23. Goldenrod, Aster, Brambles, Poison Ivy, Honeysuckle, young hardwoods

24A. Sweetgum, Cherry, Red Maple, Pin Oak, Elm, Sassafras, Persimmon, Dogwood, Tuliptree, Hornbeam

24B. Virginia Pine predominant

24C. Virginia Pine, hardwoods of 24A.

(25 only at lvy Neck)

\section{MARSH TYPES}

Fresh Marsh

26A. Grass, Rose, Black Willow

26B. Hempweed, Touch-me-not, Black Willow

Salt Marsh

*27A. Sea Myrtle, Salt Reedgrass, Salt Cordgrass

*27B. Saltmeadow Grass, Alkali Grass

*27C. Cattail, Marsh Mallow, Salt Cordgrass, Wild Bean

${ }^{\star}$ Occurs also at Ivy Neck 


\section{VEGETATION TYPES AT JAVA FARM}

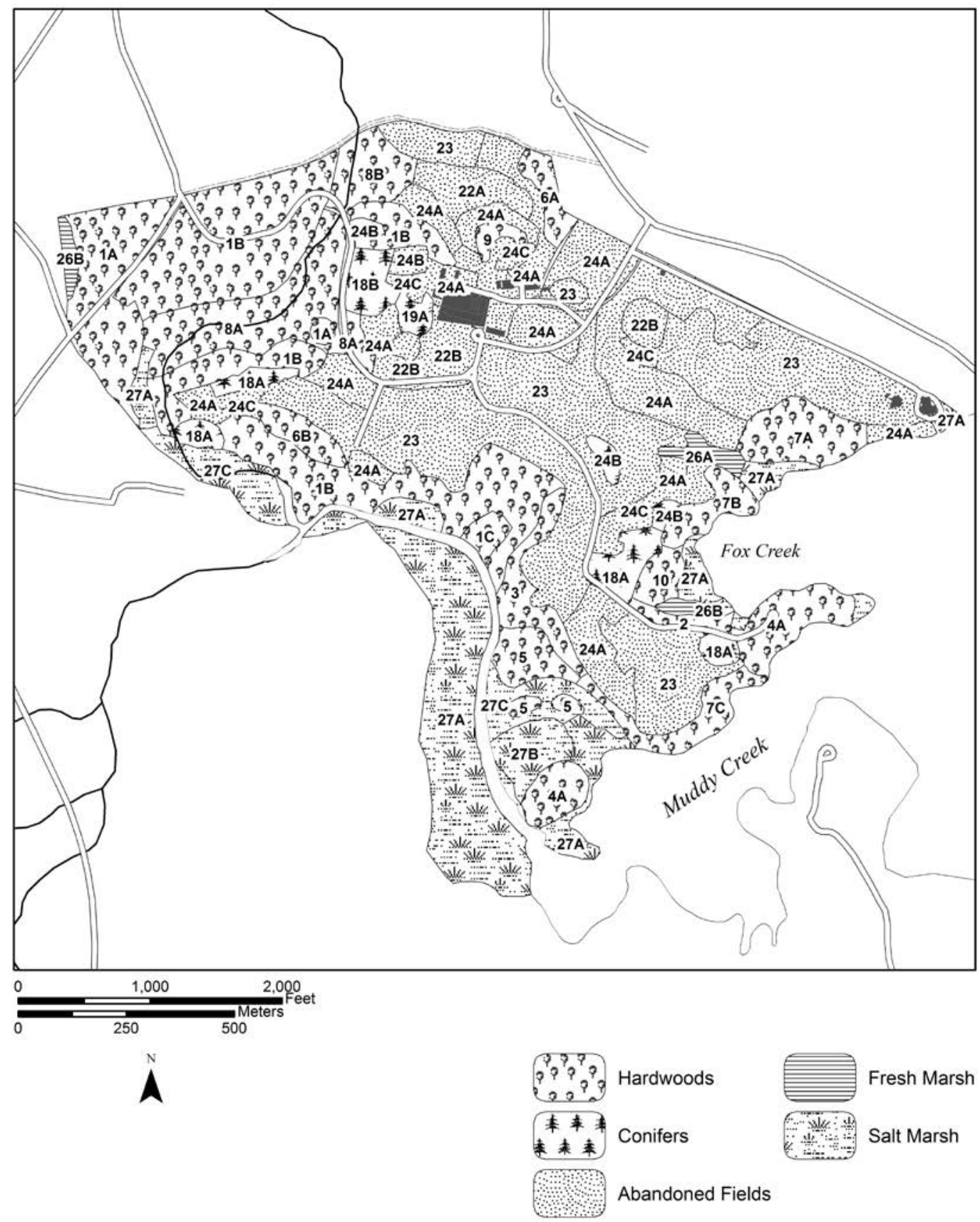


Figure 5. (Opposite)

\section{VEGETATION TYPES AT IVY NECK}

Hardwoods

( 1 to 3 only at Java Farm)

*4A. Chestnut Oak, White and Black Oaks, Hickories

4B. Chestnut Oak, White Oak, Hickories, Red Maple, Beech

4C. Chestnut Oak, Black and Scarlet Oaks, Walnut

4D. Chestnut Oak, White Oak, Virginia Pine (5 to 7C only at Java Farm)

7D. Locust, Sweetgum, Persimmon, Tuliptree, Red Maple

7E. Locust, Sweetgum, Walnut, Willow Oak

7F. Sweetgum, Walnut, Black Oak, Red Maple

(8 to 10 only at Java Farm)

11A. White Oak, Sweetgum, Locust, Red Maple

11B. White and Black Oaks, Sweetgum, Tuliptree, Hickories

11C. White and Spanish Oaks, Sweetgum, Tuliptree

12. Persimmon, Locust, Cherry, Sycamore, Maples

13A. Bitternut Hickory, White Oak, Persimmon, Tuliptree

13B. Bitternut Hickory, White and Pin Oaks, River Birch

14. Tuliptree, Sweetgum, Locust, River Birch

15. White Oak, Sycamore, Walnut, Red Maple

16. Willow Oak, Black and Blackjack Oaks, Locust

17A. Cherry, Locust, Sweetgum

17B. Cherry, Locust, Black Oak, Mockernut Hickory

\section{FOREST TYPES (continued)}

Conifers

$$
\text { (18 only at Java Farm) }
$$

*19A. Loblolly Pine, Sweetgum (pine predominant)

19B. Loblolly Pine, Sweetgum, Locust

19C. Loblolly Pine, Virginia Pine, Sweetgum, Walnut

\section{CULTIVATED FIELD TYPES}

20. Pasture

21. Cropland

\section{ABANDONED FIELD TYPES}

(22 only at Java Farm)

*23. Goldenrod, Aster, Brambles, Poison Ivy, Honeysuckle, young hardwoods

(24 only at Java Farm)

25A. Locust, Sweetgum, Cherry, Red Maple, Sassafras, Mulberry, Elm, Persimmon, Pin Oak

25B. Virginia Pine, hardwoods of 25A.

\section{MARSH TYPES}

(26 only at Java Farm)

*27A. Sea Myrtle, Salt Reedgrass, Salt Cordgrass

*27B. Saltmeadow Grass, Alkali Grass

*27C. Cattail, Marsh Mallow, Switchgrass

28. Sea Rocket, False Indigo, Salt Cordgrass, Wild Bean

\section{POND TYPES}

Small Pond: Swamp Dock, Duckweed, Water Purslane

Large Pond: Salt Cordgrass, Horned

Pondweed, Marsh Mallow, Sea Myrtle

* Occurs also at Java Farm 


\section{VEGETATION TYPES AT IVY NECK}

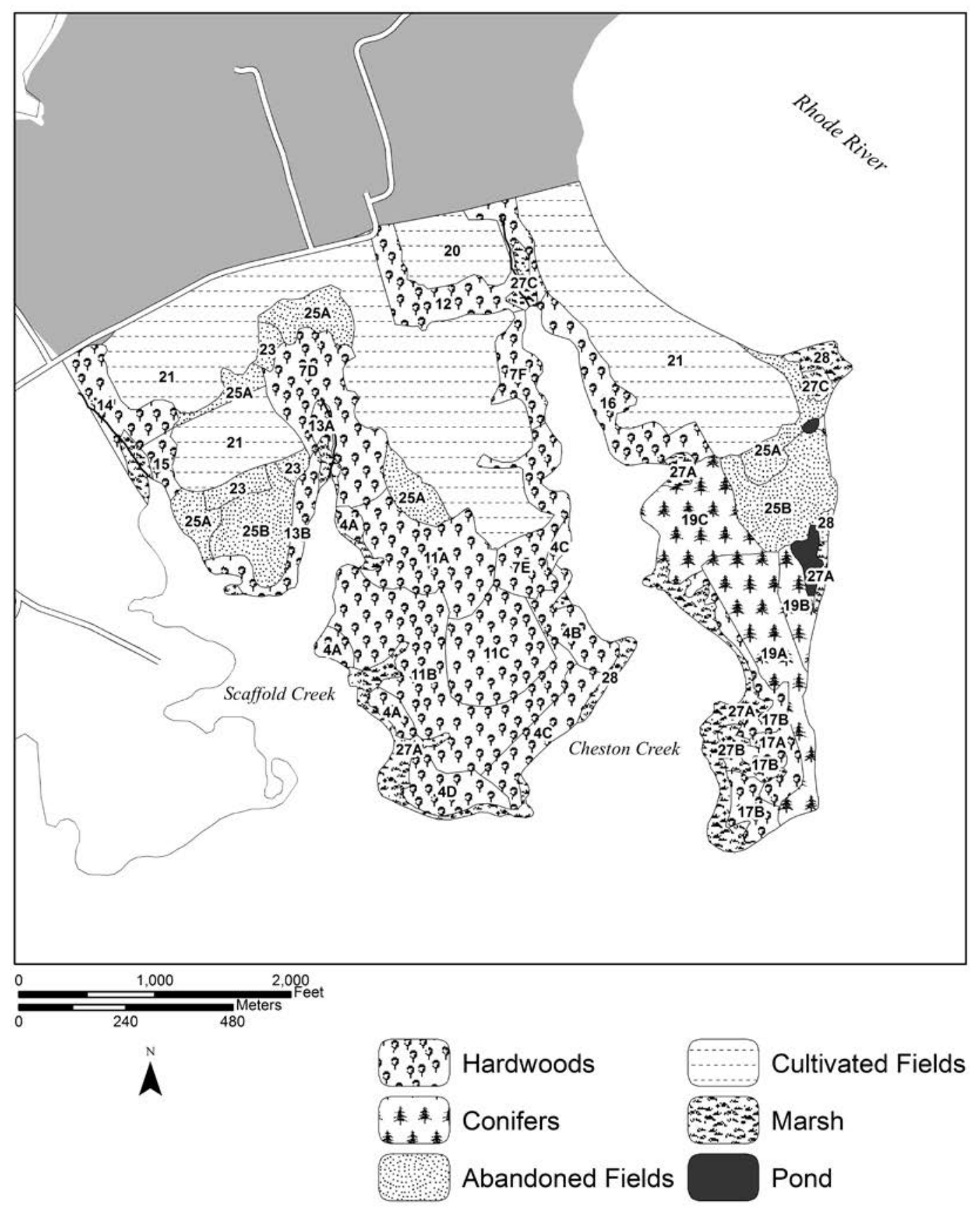


Table I.

COMPARATIVE COMPOSITION OF VEGETATION TYPES AT JAVA FARM AND IVY NECK

\begin{tabular}{c|c}
\hline Java Farm & Ivy Neck \\
\hline FOREST TYPES & FOREST TYPES \\
HARDWOODS & HARDWOODS
\end{tabular}

1A. Beech, Tuliptree.

Mature upland hardwoods on gentle westor south-facing slopes above tributary of Muddy

Creek. Canopy mainly Fagus grandifolia; less Liriodendron tulipifera, Quercus coccinea, and other hardwoods. Understory of canopy species, plus Cornus florida, Acer rubrum, etc. Shrub and ground floras include Euonymus americanus, Epifagus virginiana, and Hypoxis hirsuta (none of these found in Type 1B). Rhus radicans and Lonicera japonica virtually absent.

1B. Beech, Tuliptree, Oaks, Hickories.

Mature upland hardwoods. Fagus grandifolia and Liriodendron tulipifera throughout canopy; Liquidambar styraciflua, Quercus alba, Q. coccinea, Q. velutina, Carya tomentosa, and fewer C. falcata and C. glabra. Composition of canopy variable. Understory of canopy species, plus Cornus florida, Acer rubrum, Carpinus caroliniana, and infrequent sprouts of Castanea dentata. Shrubs include Viburnum prunifolium and infrequent Vaccinium staminium. Rhus radicans and Lonicera japonica moderately abundant. Rich herbaceous flora, most species not found in Type 1A. Herbaceous flora seems to vary with composition of the canopy. At one point, near the head of the tidal portion of Muddy Creek, the shrub and herbaceous flora resembles that of Type $4 \mathrm{~A}$
No counterpart.

11B. White and Black Oaks, Sweetgum, Tuliptree, Hickories.

Moderately mature lowland hardwoods; canopy resembles that of Type $1 \mathrm{~B}$, but contains infrequent Robinia pseudoacacia and has less Fagus grandifolia. Main difference is in understory and ground floras, which reflect poor drainage of Ivy Neck. (More Nyssa sylvatica, Viburnum prunifolium, Cercis canadensis, Lindera benzoin, Podophyllum peltatum, Arisaema atrorubens, etc.) Rhus radicans and Lonicera japonica moderately abundant.

(Types 11A and $11 \mathrm{C}$ are described on the following pages.) 
\begin{tabular}{l} 
Java Farm \\
\hline 1C. Beech, Tuliptree, White Oak, Sycamore, \\
Sweetgum.
\end{tabular}

Mature lowland hardwoods, on level bank of Muddy Creek near head of tidal portion and on bottom of tributary valley downstream. Quercus alba prominent in canopy, also Fagus grandifolia, Liquidambar styraciflua, Liriodendron tulipifera, and Platanus occidentalis. Understory of Cornus florida on bank upstream; Lindera benzoin, Viburnum prunifolium, and $\mathrm{V}$. dentatum in tributary valley.

2. Tuliptree, Black Oak, Blackgum.

Moist lowland hardwoods, on gentle north-facing slope. Canopy of Liriodendron tulipifera and Quercus velutina throughout; Nyssa sylvatica primarily on the west side, Quercus alba on the east; few Pinus virginiana. Transition to Type 4A.

Understory of Aralia spinosa, Acer rubrum, and Prunus serotina. Ground cover of Smilax rotundifolia.

3. White Oak, Black and Spanish Oaks, Sweetgum.

Mature hardwoods, on southwest-facing slope. Canopy of Quercus alba, Q. velutina, a few very big $Q$. falcata, Liquidambar styraciflua, and Nyssa sylvatica. Understory of Cornus florida, Carya tomentosa, and Liquidambar. Ground cover of Rhus radicans and Lonicera japonica.
Ivy Neck

No counterpart.

No counterpart.

No counterpart. Resembles Type $11 \mathrm{C}$ on Scaffold Peninsula (described below), but the latter has a level, poorly drained habitat. 11C. White and Spanish Oaks, Sweetgum, Tuliptree.

Moderately mature; canopy of Quercus alba, Q. falcata, Liquidambar styraciflua, Liriodendron tulipifera, Nyssa sylvatica, and a few mature Pinus virginiana.

Understory chiefly llex opaca; fewer Juniperus virginiana, Acer rubrum, Viburnum prunifolium, and Carya cordiformis. Ground cover of dense Lonicera japonica and Rhus radicans. 
Table I. (Continued)

\begin{tabular}{l} 
Java Farm \\
\hline 4A. Chestnut Oak, White and Black Oaks, \\
Hickories. \\
Mature hardwoods of coastal zone. Canopy \\
of Quercus alba, Q. prinus, Q. velutina, and \\
Carya tomentosa, with fewer C. falcata and \\
C. glabra. Mature Pinus virginiana usually few.
\end{tabular}

Understory typically of llex opaca, Cornus florida, Acer rubrum, and a few Pinus virginiana (many young pines at Fox Point); also scattered Amelanchier arborea and Viburnum acerifolium. Castanea dentata sprouts.

Ericaceous shrub layer characteristic: Kalmia latifolia, Gaylussacia baccata, G. frondosa, Vaccinium spp. Ground flora includes mosses, Hieracium venosum, and Deschampsia flexuosa. Smilax rotundifolia usually sparse; Rhus radicans and Lonicera japonica conspicuously absent.

4A. Very similar in composition to that at Java Farm, and occupies the same habitat: a narrow zone where the bank drops steeply to the Bay (probably a zone of improved drainage caused by a drop in the soil water table); the finer texture of soil at lvy Neck seems to make little difference with this type.

4B. Chestnut Oak, White Oak, Hickories, Red Maple, Beech.

Differs from Type 4A in having Fagus grandifolia and Acer rubrum in the canopy, and a more heterogeneous understory which seems transitional to inland Types $11 \mathrm{~B}$ and 11C. Understory includes Cercis canadensis, Sassafras albidum, Viburnum prunifolium, and Carya cordiformis. Rubus spp. and Campsis radicans also.

4C. Chestnut Oak, Black and Scarlet Oaks, Walnut.

Canopy and understory are more varied than in either 4A or 4B; has less Quercus prinus and almost no ericaceous shrubs. New canopy species include $Q$. coccinea, Juglans nigra, Liquidambar styraciflua, Liriodendron tulipifera, Asimina triloba, etc.

4D. Chestnut Oak, White Oak, Virginia Pine.

Differs from 4A in having infrequent Pinus virginiana in canopy Hardwoods formerly lumbered. Pine abundant in understory, with Acer rubrum and Liquidambar styraciflua. llex opaca and ericaceous shrubs infrequent. 


\begin{tabular}{|c|c|}
\hline Java Farm & Ivy Neck \\
\hline $\begin{array}{l}\text { 5. Spanish and Willow Oaks, Sweetgum, } \\
\text { Tuliptree. }\end{array}$ & No counterpart. \\
\hline $\begin{array}{l}\text { Mature lowland hardwoods adjacent Hog } \\
\text { Island salt marsh. Canopy of Quercus } \\
\text { falcata, Q. phellos, Liquidambar } \\
\text { styraciflua, and Liriodendron tulipifera. } \\
\text { Understory of Cornus florida, } \\
\text { Liquidambar, Aralia spinosa, and } \\
\text { Viburnum dentatum; infrequent Quercus } \\
\text { stellata and Pinus virginiana. Seems to } \\
\text { be invading drier areas of salt marsh. }\end{array}$ & \\
\hline $\begin{array}{l}\text { 6A. Walnut, Sweetgum. } \\
\text { Mature stand, probably persistent } \\
\text { from cultivation, on steep east- and } \\
\text { west-facing slopes of ridge. Canopy } \\
\text { of Juglans nigra only on east side; } \\
\text { with Pyrus communis, Liquidambar } \\
\text { styraciflua, and Sassafras albidum } \\
\text { on west side. Understory of Lindera } \\
\text { benzoin on east slope, Rubus on west. } \\
\text { Dense Lonicera japonica on both sides. }\end{array}$ & $\begin{array}{l}\text { No counterpart. Walnut grows infrequently along } \\
\text { both sides of Cheston Creek estuary, in Types } \\
4 C, 7 E, 7 F \text {, and 19C, and on upper Scaffold } \\
\text { Creek estuary in Type 15, but not abundantly } \\
\text { enough to characterize a stand. }\end{array}$ \\
\hline $\begin{array}{l}\text { 6B. Walnut, Sweetgum, Spanish Oak, } \\
\text { Beech. }\end{array}$ & No counterpart. \\
\hline $\begin{array}{l}\text { Moderately mature, at top of south- } \\
\text { facing slope. Canopy of Liquidambar } \\
\text { styraciflua, Juglans nigra, Quercus } \\
\text { falcata, Fagus grandifolia, and Platanus } \\
\text { occidentalis. Understory of Lindera } \\
\text { benzoin and Cornus florida. Lonicera } \\
\text { japonica on ground. }\end{array}$ & \\
\hline
\end{tabular}


Table I. (Continued)

Java Farm

7A. Locust, Sweetgum, Elm.

Immature, on south- and southeastfacing slopes overlooking Fox Creek estuary. Canopy of Robinia pseudoacacia, Liquidambar styraciflua, and UImus americana; fewer Sassafras albidum and Quercus velutina, the latter mostly on the lower slopes. Understory of canopy species. Dense ground cover of Lonicera japonica.

Also contains scattered Acer rubrum and Cornus florida.

7B. Locust, Black Oak, Sweetgum, Red Maple, Sassafras.

Immature, on south-facing slope near head of Fox Creek estuary. Canopy of Robinia pseudoacacia and Liquidambar styraciflua, with fewer Quercus velutina, Acer rubrum, Cornus florida, and Sassafras albidum. Understory of canopy species, plus Ulmus americana. Ground cover of Lonicera japonica and Rhus radicans.

7C. Locust, Sweetgum, Tuliptree, Sycamore.

Moderately mature, along the shore of Muddy Creek estuary. Canopy chiefly Robinia pseudoacacia, especially near Hog Island salt marsh; also includes Liquidambar styraciflua, Cornus florida, Liriodendron tulipifera, and Platanus occidentalis near the eastern end. Understory of canopy species. Ground layer of Rhus radicans, Smilax rotundifolia, and Lonicera japonica.
Ivy Neck

Types 7D, 7E, and 7F at lvy Neck resemble Types 7A and 7B at Java Farm in species composition. Chief difference is in the habitat: from moderately steep south-facing slope and fine sandy loam soil at Java Farm to poorly drained level lowland and silt loam soil at lvy Neck.

7D. Locust, Sweetgum, Persimmon, Tuliptree, Red Maple.

Immature, covers watershed of east fork of Scaffold Creek. Canopy seems to contain no Quercus velutina but does include Diospyros virginiana, Nyssa sylvatica, and Platanus occidentalis. Otherwise the same as at Java Farm (Robinia, Liquidambar, Ulmus )

Understory highly variable, includes Sassafras albidum, Cornus florida, Lindera benzoin, Viburnum prunifolium, Acer rubrum, and Carya cordiformis. Shrub and ground layers of Rubus spp., Rhus radicans, and Lonicera japonica.

Type 11A, described on the next page, seems to be a transition between 7D above and $11 \mathrm{~B}$ (described on p. 16).

7E. Locust, Sweetgum, Walnut, Willow Oak. Immature, covers west bank of Cheston Creek estuary north of Type 4B. Resembles Type 7F (p. 21) and differs from other variations of Type 7 in having Juglans nigra in the canopy.

Canopy of Robinia pseudoacacia, Liquidambar styraciflua, Juglans nigra, and Quercus phellos. Understory of Robinia pseudoacacia, Cornus florida, and Sassafras albidum. Ground layer Lonicera japonica and Smilax rotundifolia. 


\begin{tabular}{|c|c|}
\hline Java Farm & Ivy Neck \\
\hline No counterpart. & $\begin{array}{l}\text { 7F. Sweetgum, Walnut, Black Oak, Maple. } \\
\text { Immature, adjoins Type 7E. Canopy has } \\
\text { little or no Robinia, added Quercus velutina, } \\
\text { Salix nigra, Acer rubrum, and Platanus } \\
\text { occidentalis. Understory of Viburnum } \\
\text { prunifolium, Prunus serotina, and Cercis } \\
\text { canadensis. Ground layer as in Type 7E. }\end{array}$ \\
\hline No counterpart. & $\begin{array}{l}\text { 11A. White Oak, Sweetgum, Locust, Red } \\
\text { Maple. } \\
\text { Immature, apparently a transition between } \\
\text { Types 7D and 11B. Canopy of Quercus } \\
\text { alba, Liquidambar styraciflua, Nyssa } \\
\text { sylvatica, Acer rubrum, and Carya spp.; } \\
\text { Robinia pseudoacacia becomes infrequent } \\
\text { southward; Ulmus americana infrequent. } \\
\text { Understory varied, includes Rhus typhina, } \\
\text { Ailanthus altissima, Cornus florida, Viburnum } \\
\text { prunifolium, Morus alba, Cercis canadensis, } \\
\text { etc. Ground cover of Lonicera japonica and } \\
\text { Rhus radicans. }\end{array}$ \\
\hline
\end{tabular}

8A. White Ash, Sycamore.

Moderately mature, follows bottom of Muddy Creek valley above tidal limit. Canopy mostly Fraxinus americana, fewer Platanus occidentalis, few species of Type 8B below. Understory of Lindera benzoin, with few Acer rubrum, Fraxinus, Liquidambar, Ulmus americana, and Carpinus caroliniana. Ground layer of Rubus spp., Vitis spp., and $R$ hus radicans. Herbaceous flora mostly Impatiens capensis, Podophyllum peltatum, and assorted grasses.
12. Persimmon, Locust, Cherry, Sycamore, Maples.

Moderately mature, follows drainage of Cheston Creek above tidal limit. Persimmon and cherry may be cultivated. Understory inhibited by browsing cattle.

Canopy of Diospyros virginiana, Robinia pseudoacacia, Prunus serotina, Platanus occidentalis, Acer rubrum, A. saccharinum, A. negundo, Liquidambar styraciflua, Nyssa sylvatica. Understory of Viburnum prunifolium, Salix nigra, Cercis canadensis, Celtis occidentalis. Ground layer chiefly grass, or forbs in wet areas. 
Table I. (Continued)

Java Farm
8B. White Ash, Sycamore, Red Maple, Elm,
Sweetgum.
Similar to Type 8A but farther upstream.
More heterogeneous canopy includes Acer
rubrum, Ulmus americana, Liquidambar
styraciflua, and Quercus palustris.
Understory as in Type 8A, with more Ulmus
americana and Carpinus caroliniana. Ground
layer same.

9. Tuliptree, Sweetgum, Red Maple,

No counterpart.

Sassafras.

Immature, covers north- and west-facing slopes of hill overlooking wet meadow.

Canopy of Liriodendron tulipifera, Liquidambar styraciflua, Acer rubrum, and Sassafras albidum. Understory similar, plus llex opaca, Prunus serotina, Nyssa sylvatica, Cornus florida, Viburnum prunifolium. Ground layer of Lonicera japonica.

10. Dogwood, Sweetgum, Red Maple, Sassafras.

Immature, covers east-facing slope at head of Fox Creek estuary; merges with Virginia pine stand (Type 18A) on upper slope. Canopy of Cornus florida, Acer rubrum, Sassafras albidum, Liquidambar styraciflua, Pinus virginiana, few Fagus grandifolia and Morus rubra. Understory similar. Ground layer of Smilax rotundifolia, stopping sharply at edge of pine canopy.

Ivy Neck

No counterpart. 


\begin{tabular}{|c|c|}
\hline Java Farm & Ivy Neck \\
\hline No counterpart. & $\begin{array}{l}\text { 13A. Bitternut Hickory, White Oak, } \\
\text { Persimmon, Tuliptree. } \\
\text { Moderately mature but canopy thin. Lower } \\
\text { valley of Scaffold Creek (east fork) above } \\
\text { tidal limit. Canopy of Carya cordiformis, } \\
\text { fewer Quercus alba, Platanus occidentalis, } \\
\text { Diospyros virginiana, and Liriodendron } \\
\text { tulipifera. Understory thin, of Morus rubra, } \\
\text { Juglans nigra, Ulmus rubra, Quercus } \\
\text { palustris, etc. Shrub and ground layers very } \\
\text { dense, of Rubus spp., Smilax rotundifolia, } \\
\text { and Lonicera japonica. }\end{array}$ \\
\hline $\begin{array}{l}\text { No counterpart. (Habitat of Type } 7 \mathrm{C} \\
\text { resembles that of } 13 \mathrm{~B} \text { except that } 7 \mathrm{C} \text { has } \\
\text { fine sandy loam soil; } 13 \mathrm{~B} \text { has silt loam). }\end{array}$ & $\begin{array}{l}\text { 13B. Bitternut Hickory, White and Pin Oaks, } \\
\text { River Birch. } \\
\text { Moderately mature, borders field between } \\
\text { forks of Scaffold Creek. Canopy of Carya } \\
\text { cordiformis, Quercus alba, Q. palustris. } \\
\text { Understory of Cornus florida and Viburnum } \\
\text { prunifolium, few Quercus alba, Q. stellata, } \\
\text { Carya glabra, and Fagus grandifolia. Ground } \\
\text { layer of mixed grasses, few woody vines. }\end{array}$ \\
\hline No counterpart, & $\begin{array}{l}\text { 14. Tuliptree, Sweetgum, Locust, River } \\
\text { Birch. } \\
\text { Immature, covers drainage of west fork } \\
\text { of Scaffold Creek. Canopy of Liriodendron } \\
\text { tulipifera, less Liquidambar styraciflua, } \\
\text { Robinia pseudoacacia, Betula nigra, and } \\
\text { Juniperus virginiana. Understory of Viburnum } \\
\text { spp., Carya cordiformis, Quercus velutina, } \\
\text { and Q. palustris. Ground layer of Rhus } \\
\text { radicans. }\end{array}$ \\
\hline
\end{tabular}


Table I. (Continued)

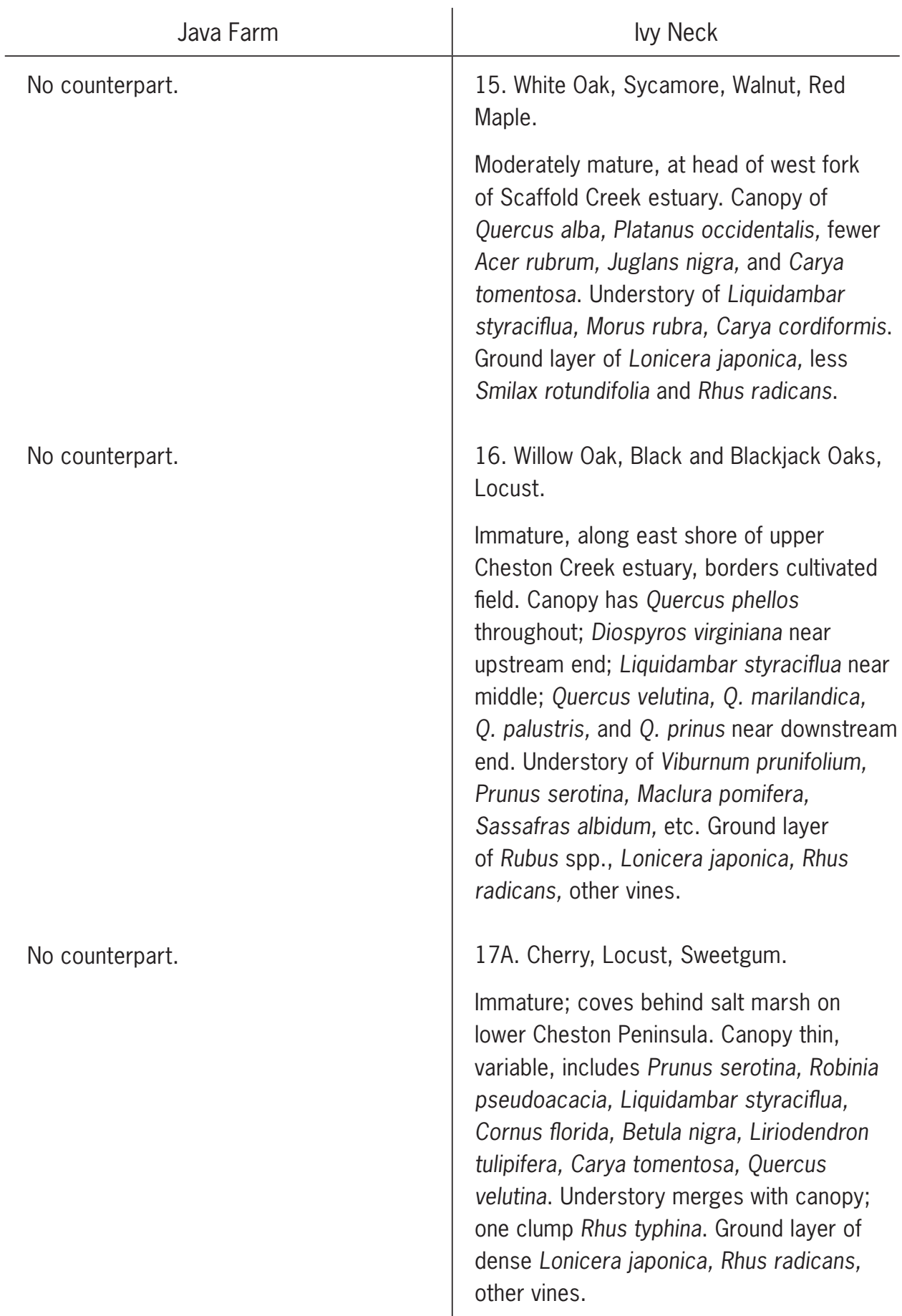




\begin{tabular}{|c|c|}
\hline Java Farm & Ivy Neck \\
\hline No counterpart. (Habitat like that of Type 4). & $\begin{array}{l}\text { 17B. Cherry, Locust, Black Oak, Mockernut } \\
\text { Hickory. } \\
\text { Immature; on Cheston Point and low } \\
\text { headlands adjoining salt marsh. Canopy } \\
\text { of Prunus serotina, Robinia pseudoacacia, } \\
\text { Quercus velutina, Carya tomentosa, Q. alba, } \\
\text { Betula nigra. Understory on Cheston Point of } \\
\text { Liquidambar styraciflua and Ulmus americana; } \\
\text { on headlands of llex opaca and Viburnum } \\
\text { prunifolium. Ground layer of Smilax rotundifolia } \\
\text { on one headland; Rubus spp., Rhus radicans, } \\
\text { and Lonicera japonica elsewhere. }\end{array}$ \\
\hline CONIFERS & CONIFERS \\
\hline $\begin{array}{l}\text { 18A. Virginia Pine predominant. } \\
\text { Moderately mature; scattered stands usually } \\
\text { on upper slopes or summits, exception at } \\
\text { head of tidal part of Muddy Creek. Canopy of } \\
\text { Pinus virginiana, may have few Liquidambar } \\
\text { styraciflua, stand at bend of Fox Point Rd. } \\
\text { has few Quercus velutina and Q. marilandica. } \\
\text { Understory of mixed hardwoods, chiefly } \\
\text { Liquidambar, Acer rubrum, etc. Ground layer } \\
\text { usually Lonicera japonica. }\end{array}$ & No counterpart. \\
\hline
\end{tabular}


Table I. (Continued)

\begin{tabular}{l|l}
\multicolumn{1}{c|}{ Java Farm } & \multicolumn{1}{c}{ Ivy Neck } \\
\hline $\begin{array}{l}\text { 18B. Virginia Pine, Sweetgum, Tuliptree, } \\
\text { Hickories. }\end{array}$ & $\begin{array}{l}\text { No true counterpart; few mature Pinus } \\
\text { virginiana remain of former stand on } \\
\text { Scaffold Peninsula (now hardwood Type }\end{array}$ \\
A mixture of Type 18A above and hardwood & $\begin{array}{l}11 \mathrm{C} \text { ). This area resembles Type 18B to the } \\
\text { extent that Type 11C resembles Type 1B at } \\
\text { Type 1B (p. 16). West-facing slope of ridge } \\
\text { with water tank. Canopy on lower slope of } \\
\text { Pinus virginiana and Liquidambar, latter gives }\end{array}$
\end{tabular}
way upslope to Liriodendron tulipifera, Carya tomentosa, and few Quercus velutina. Mature pine only on slope, summit has young pine and hardwood stands (Types 24A, B, C). Understory all over slope of Liriodendron, Acer rubrum, Sassafras albidum, and few Pinus virginiana. Shrubs only on lower slope: Vaccinium spp., Myrica cerifera. Ground layer below of Mitchella repens and Lycopodium complanatum, of Rhus radicans on upper slope.

19A. Loblolly Pine, Sweetgum (pine predominant).

Mature; on south-facing slope of ridge with water tank, described for Type 18B. Canopy of Pinus taeda and Liquidambar styraciflua. Understory of Liquidambar, Liriodendron tulipifera, and Acer rubrum. Ground layer of Rhus radicans.

Also one mature Pinus taeda at headquarters area and one at west end of Fox Point Road; few young ones in old fields (Type 23).

No counterpart.

19A. Loblolly Pine, Sweetgum (pine predominant).

Mature; planted in 1933 on Cheston Peninsula. Very dense; many trees down, rest with thin crowns; reproduction at stand borders. Canopy of Pinus taeda, few Liquidambar styraciflua and Liriodendron tulipifera. Understory of dense Liquidambar, Liriodendron, Cornus florida; fewer Prunus serotina, Ulmus americana, and Quercus velutina. Ground layer of dense Rhus radicans and Lonicera japonica.

19B. Loblolly Pine, Sweetgum, Locust. Immature; triangular area on Cheston Peninsula between $19 \mathrm{~A}$ and coast. Thin canopy of Pinus taeda, Liquidambar styraciflua and Robinia pseudoacacia. Dense understory same as for Type 19A, with few young Pinus taeda. Dense ground layer of Rhus radicans, Lonicera japonica, Campsis radicans, Vitis $\mathrm{sp}$. 


\begin{tabular}{l|l}
\multicolumn{1}{|c|}{ Java Farm } & \multicolumn{1}{c}{ Ivy Neck } \\
\hline No counterpart. & $\begin{array}{l}\text { 19C. Loblolly Pine, Virginia Pine, Sweetgum, } \\
\text { Walnut. } \\
\text { Immature; on Cheston Peninsula between }\end{array}$ \\
& Type 19A and cultivated field. Canopy of \\
& Liquidambar styraciflua, fewer Pinus taeda, \\
& P. virginiana, and Juglans nigra. Understory \\
& of Acer rubrum, Carya cordiformis, Ilex \\
& opaca, Nyssa sylvatica, Quercus alba, \\
& Q. palustris, and Q. phellos. Shrubs: Myrica \\
& cerifera, Viburnum prunifolium. Ground layer \\
& of Rhus radicans and Lonicera japonica. \\
\hline
\end{tabular}

No counterpart.

No counterpart.

\section{CULTIVATED FIELD TYPES}

20. Pasture.

Single pasture, grazed by dairy herd. Gentle south-facing slope, bordered on 3 sides by Cheston Creek drainage. Flora of Andropogon virginicus, Digitaria ischaemum, Trifolium repens, Taraxacum officinale, other forbs.

21. Cropland.

Cultivated to corn, barley, wheat, and sorghum. Wild flora mostly Ipomoea purpurea, I. hederacea; also (vernal): Stellaria media, Draba verna, Lepidium spp., Barbarea spp., Cerastium spp., etc. and (autumnal) Aster spp.; restricted or infrequent species: Datura stramonium, Echinochloa pungens; Setaria lutescens often abundant near borders. 
Table I. (Continued)

\begin{tabular}{|c|c|}
\hline Java Farm & Ivy Neck \\
\hline & $\begin{array}{l}\text { One field on Scaffold Peninsula, described } \\
\text { in text as "abandoned wheatfield" (since } \\
\text { recultivated) was fallow } 3 \text { yrs. (1963-66); } \\
60 \text { species were found there in 1966, } \\
\text { especially Oenothera biennis, O. fruticosa, } \\
\text { Gnaphalium obtusifolium, Cerastium } \\
\text { viscosum, Dianthus armeria, Hypericum } \\
\text { perforatum, Ambrosia artemisiifolia. Locally } \\
\text { dense Trifolium pratense and T. hybridum. } \\
\text { Woody species found under forbs: Campsis } \\
\text { radicans dense; Quercus velutina, Prunus } \\
\text { serotine, and Vitis sp. few. }\end{array}$ \\
\hline FORMERLY CULTIVATED FIELD TYPES & FORMERLY CULTIVATED FIELD TYPES \\
\hline $\begin{array}{l}\text { 22A. Reed Canary Grass. } \\
\text { Former pasture in valley north of } \\
\text { headquarters area, marshy at the west } \\
\text { end, drains into Muddy Creek. Very dense } \\
\text { Phalaris arundinacea throughout; Juncus } \\
\text { effusus, Scirpus rubricosus, Polygonum } \\
\text { pensylvanicum, and P. sagittatum at marshy } \\
\text { end; few clumps of young Acer rubrum, } \\
\text { Liquidambar styraciflua, and Rosa spp. } \\
\text { Invasion from outside slow. }\end{array}$ & $\begin{array}{l}\text { No counterpart. (When the field described } \\
\text { above was abandoned, its north end } \\
\text { adjacent Cheston Creek drainage had } \\
\text { marshy species like that of western part of } \\
\text { Type 22A.) }\end{array}$ \\
\hline $\begin{array}{l}\text { 22B. Kentucky Bluegrass. Small areas } \\
\text { of dense Poa pratensis turf in former } \\
\text { cultivated fields, being overrun by Rhus } \\
\text { radicans. Associated forbs: Gnaphalium } \\
\text { obtusifolium, Erigeron canadensis, } \\
\text { Asclepias syriaca, Solanum carolinense, } \\
\text { Cirsium discolor. }\end{array}$ & No counterpart. \\
\hline
\end{tabular}




\begin{tabular}{l|l}
\multicolumn{1}{c|}{ Java Farm } & \multicolumn{1}{c}{ Ivy Neck } \\
\hline $\begin{array}{l}\text { 23. Goldenrod, Aster, Brambles, Poison Ivy, } \\
\text { Honeysuckle, scattered young hardwoods. }\end{array}$ & $\begin{array}{l}\text { 23. Goldenrod, Aster, Brambles, Poison Ivy, } \\
\text { Honeysuckle, scattered young hardwoods. }\end{array}$ \\
$\begin{array}{l}\text { Very heterogeneous vegetation throughout } \\
\text { most former cultivated fields. Forbs often }\end{array}$ & $\begin{array}{l}\text { Similar to that at Java Farm but with fewer } \\
\text { vines, more grass and forbs. Merges with }\end{array}$ \\
more abundant than woody vines but often & Types 25A, 25B. Grasses include Setaria \\
less so. Principal forbs: Solidago altissima, & lutescens, Andropogon virginicus.
\end{tabular}

S. graminifolia, Aster pilosus, A. dumosus, Eupatorium serotinum, Rubus occidentalis, Lespedeza cuneata, Rosa micrantha, R. multiflora, Allium vineale. Early spring forbs: Barbarea spp., Draba verna, Lepidium spp., etc. Principal vines: Rhus radicans, Lonicera japonica, Campsis radicans, Vitis vulpina. Many less frequent species. Trees scattered or locally dense; see Type 24A.

24A. Sweetgum, Cherry, Red Maple, Pin Oak, Elm, Sassafras, Persimmon, Dogwood, Tuliptree, Hornbeam.

Very dense young stands, often predominantly Liquidambar styraciflua but often heterogeneous. Covers parts of old fields and usually merges with Type 23. Principal species: Liquidambar, Prunus serotina, Acer rubrum, Quercus falcata, Sassafras albidum, Cornus florida. Less frequent: Liriodendron tulipifera (central upland), Diospyros virginiana (lowland), Ulmus americana, U. rubra (near pier), Carpinus caroliniana (forest border), Celtis occidentalis (all over). Understory usually none. Ground layer of dense Lonicera japonica and Rhus radicans in all stands.

Special cases:

South-facing slope at north border (adjacent Type 22A) is an old orchard; canopy of Liquidambar, Liriodendron, and Pyrus malus. Understory of Rubus spp.

25A. Locust, Sweetgum, Cherry, Red Maple, Sassafras, Mulberry, Elm, Persimmon, Pin Oak.

Corresponds to Type 24A but has Robinia pseudoacacia usually abundant; Liriodendron, Carpinus, Ulmus rubra, and Celtis infrequent to absent.

Special cases:

Stand at head of Scaffold Creek drainage includes Nyssa sylvatica, Platanus occidentalis, Carya cordiformis, and shrubs Viburnum prunifolium and Sambucus canadensis.

Stand on Cheston Peninsula below Type 21 includes dense understory of Myrica cerifera.

Hedgerows between cultivated fields usually contain Celtis occidentalis, Liquidambar styraciflua, Maclura pomifera, Acer rubrum, or Viburnum prunifolium; also Campsis radicans. 
Table I. (Continued)

Java Farm
West-facing slope down to Muddy Creek
near head of tidal portion has very dense
Carpinus canopy; so does stand at end of
Springhouse Road.

24B. Virginia Pine predominant.

Dense young stands of Pinus virginiana.

Two occur on summit and west-facing slope of ridge west of headquarters area, one in old field on northeast-facing slope near Fox Point Rd. Few Liquidambar in stands, no understory; ground cover of Rhus radicans and Lonicera japonica.

Pine also infrequent in Type 23.

24C. Virginia Pine, hardwoods of $24 \mathrm{~A}$.

Simultaneous growth of young Pinus virginiana and Liquidambar styraciflua in old fields. Stand overlooking head of Fox Creek estuary also has Robinia pseudoacacia, Quercus palustris, Cornus florida, and Sassafras albidum. Stand at west end of field overlooking Muddy Creek at southwest corner of Farm has Liriodendron tulipifera and Aralia spinosa.

Ground cover of Rhus radicans and Lonicera japonica.

\section{MARSH TYPES}

\section{Freshwater Marsh}

26A. Grass, Rose, Black Willow.

Freshwater portion of marsh at mouth of Fox Creek drainage, separated from salt marsh by hedge of Rosa palustris and Smilax rotundifolia. Assorted grasses, scattered Salix nigra, clumps of Rosa palustris, Impatiens capensis, and Scirpus spp.
No counterpart.

Ivy Neck

25B. Virginia Pine, hardwoods of 25A.

Infrequent occurrence of Pinus virginiana

throughout young hardwood canopies

between forks of Scaffold Creek estuary

and on Cheston Peninsula between pine

stand and cultivated fields.

Ground cover of Rhus radicans and Lonicera japonica.

\section{MARSH TYPES}

No freshwater marshes occur at lvy Neck. 


\begin{abstract}
Java Farm
26B. Hempweed, Touch-me-not, Black Willow.

Two sites: marshy streambed at western border of Farm and at base of Fox Point peninsula adjoining Fox Creek estuary. Both sites have dense Impatiens capensis and Mikania scandens, and scattered Salix nigra and Smilax rotundifolia. Scutellaria lateriflora and Rosa spp. grow at the first site, Sambucus canadensis at the second.
\end{abstract}

\section{Salt Marsh}

27A. Sea Myrtle, Salt Reedgrass, Salt Cordgrass.

Covers the wettest and apparently most saline marsh areas and follows tide channels into apparently less saline areas. Chiefly Baccharis halimifolia, Spartina cynosuroides, S. alterniflora; less abundant species: Iva frutescens, Aster subulatus, A. tenuifolius, Limonium carolinianum, Phragmites communis.

27B. Saltmeadow Grass, Alkali Grass. Covers more sheltered, inland areas of large marshes; often absent from smaller ones. Chiefly Spartina patens, Distichlis spicata; less abundant species: Solidago sempervirens, Eleocharis halophila.

27C. Cattail, Marsh Mallow, Switchgrass.

Occurs at landward margins of marshes or throughout less saline ones. Chiefly Typha latifolia, T. angustifolia, Hibiscus palustris, Kosteletzkya virginica, Panicum virgatum; less abundant species: Acnida cannabina, Pluchea camphorata.
Ivy Neck

\section{Salt Marsh and Beach}

27A. Sea Myrtle, Salt Reedgrass, Salt Cordgrass.

Same as at Java Farm; Spartina cynosuroides infrequent, only at edge of water.

27B. Saltmeadow Grass, Alkali Grass.

Same as at Java Farm but areas often too small to map.

27C. Cattail, Marsh Mallow, Switchgrass.

Same as at Java Farm, but areas often too small to map. 
Table I. (Continued)

\begin{tabular}{l|l}
\multicolumn{1}{c|}{ Java Farm } & \multicolumn{1}{c}{ Ivy Neck } \\
\hline Tidal portion of Muddy Creek has gradient of & Salt marsh at head of Cheston Creek \\
decreasing salinity. Species there not cited & estuary has several species cited for tidal \\
above: Scirpus americanus, S. robustus & Muddy Creek. \\
(Hog I. Marsh); Atriplex patula, Dryopteris &
\end{tabular}

thelypteris (with Typha), Polygonum

hydropiperoides, Asclepias incarnata, and

Mikania scandens (at upstream end).

Trees occasionally found in salt marshes include Diospyros virginiana and Juniperus

virginiana at several locations, Robinia

pseudoacacia at Sand Point, Quercus falcata

and Q. phellos at Hog Island marsh, and Salix nigra at head of Fox Creek. The last three

species occur near the dry land border.

No counterpart.

28. Sea Rocket, False Indigo, Salt

Cordgrass, Wild Bean

Sandy beach along Cheston Peninsula, and sandbars of salt marshes. Species include Cakile edentula, Amorpha fruticosa, Spartina alterniflora, Strophostyles helvola, Cenchrus tribuloides, Ammophila arenaria, Asparagus officinale.

Baccharis halimifolia dense on Sand Point.

\section{POND TYPES}

No counterpart (types $26 \mathrm{~A}$ and $26 \mathrm{~B}$ may be temporarily innundated, but true ponds are lacking.)

No counterpart.

\section{POND TYPES}

29A. Small pond on Cheston Peninsula, separated from Rhode River by an apparently stable sandbar. Vegetation indicates low salinity at present; species include Rumex verticillatus, Echinochloa walteri, Sparganium eurycarpum, Lemna minor, Spirodela polyrhiza, Ludwigia palustris.

29B. Larger pond on Cheston Peninsula, separated from Rhode River by an eroding sandbar. Vegetation indicates a salinity equivalent to that of marshes; species include Spartina alterniflora, Zannichellia palustris, Hibiscus palustris, Baccharis halimifolia, Pluchea camphorata. 


\section{Structure of the Checklist and Keys}

he checklist includes 553 species of vascular cryptogams and phanerogams, distributed among 98 families. They were collected at Java Farm in the autumn of I9 65 and at both Java Farm and Ivy Neck during the growing seasons of I966 and I967. Collection will be started at Corn Island in I968 and continued elsewhere in coordination with studies of vegetation development. An appendix (p. 225) is provided for the addition of new species. It now contains eight species collected during the preparation of this checklist. When the Center becomes further developed the checklist will be revised for final publication.

The families represented in the checklist are indexed on page 40 and arranged according to the Engler and Prantl system. When a family is represented by more than one species artificial keys are provided. Although many of the keys are set up in a form similar to that used in Gray's Manual of Botany, 8th edition (Fernald, I950), the keys developed for this checklist are less extensive than Fernald's, since they are restricted to the flora of the Center and its near vicinity. In addition, the terminology has been simplified and in some of the more complex keys the sequence has been revised.

The keys in three families have been adapted from those of local taxonomic studies compiled for the Washington-Baltimore area, which includes Anne Arundel County (Hermann, I946). These three families are the Gramineae (Gilman, I957), the Cyperaceae (Hermann, I94I), (O’Neill, I94I), and the Umbelliferae (Dayton, I942). The adapted keys were modified to exclude species having a strictly Piedmont distribution and those restricted to habitats which do not occur at the Chesapeake Bay Center. Entirely new keys were prepared for some of the more complex taxa which have limited representation at the Center.

There are two reasons for any similarities present between keys in the literature cited above and keys in the checklist. First, the dependability of the characters in the keys in the literature. Second, the need of a framework to facilitate the anticipated periodic revision of the keys as additional species are collected. Such 
revisions will be accomplished more readily if the organization of the checklist keys is designed to allow for them.

Therefore, when a couplet in one of the keys in the literature separates a genus or species which has in fact been collected at the Center from one not yet discovered there but expected to occur in the vicinity, both halves of the couplet are included in the checklist key but the word "expected" follows the unused half and the taxa to which it refers are named in parentheses. This practice appears justifiable in view of the continued investigations to be conducted at the Center and the rich and fluctuating flora of the surrounding county, described by Stieber (1967). Stieber notes a great intermingling of northern, southern, and introduced species, the whole assembly subjected to widespread human disturbance. From field collections west of the Chesapeake Bay Center and an examination of the District of Columbia and Vicinity collection in the U. S. National Herbarium, Stieber has compiled a checklist of 647 vascular species for the county. His list cites 316 species not yet discovered at the Chesapeake Bay Center; the writer has collected at the Center 209 species which are not included on Stieber's list for the county. This complementary collection reinforces Stieber's observation on the need for further systematic floristic study of the region, and supports the writer's provision for the inclusion of additional taxa in the keys of the present list.

To prevent the checklist keys from becoming top-heavy with "expected" taxa, the writer has used the following criteria in selecting them:

I. They must be named as established in F. J. Hermann's A Checklist of Plants in the Washington-Baltimore Area, 2nd edition (Hermann, I946).

2. They must be described in the available literature as having a distribution which includes the Maryland Coastal Plain, and must not be restricted to swamps or other habitats which do not occur at the Center.

3. They must not be too numerous to keep the keys relatively simple and short.

This system of including "expected" taxa in the keys has already facilitated the identification of several species listed in Appendix I. Further research at the Center may reveal other taxa not yet listed as "expected." The identification of new taxa may be confirmed by the use of Gray's Manual.

\section{Nomenclature}

The nomenclature of the checklist follows that of Gray's Manual of Botany, 8th edition, except in the family Gramineae; there, Gilman (1957) follows the nomenclature of A. S. Hitchcock's Manual of the Grasses of the United States. Whenever the nomenclature used conflicts with that of The New Britton and Brown Illustrated Flora (Gleason, 1952), the names from the latter are given in parentheses. Common names of species given by Gray's Manual are placed opposite the scientific names. 
Additional common names used by residents of the vicinity of the Chesapeake Bay Center are distinguished by the letter "(L)," for "local," or are listed separately in Appendix III.

\section{Annotations}

The ecological annotation which accompanies each species has five categories: the general location (Java Farm or Ivy Neck), the estimated abundance of the species, its exact collection site or specific habitat, the dominant or canopy species of the collection site, and some of the associate species observed in the same location and stratum.

The estimated abundance of an entry indicates the ease or rediscovering that species in the field; the four categories are:
Abundant (plentiful throughout the habitat)
Moderately abundant (evenly distributed but not plentiful)
Infrequent (thinly scattered)
One station (a single plant or very small, compact group)

The last category is preferred to "Rare" or "Local" because the Center has not yet been studied in sufficient detail to permit a safe assumption concerning the restriction of distribution of species. Additional annotation data are being sought and are listed in Appendix II, When the checklist is revised a more precise evaluation will be made.

The specific location of a species is described in relation to existing landmarks and to the numbered grid now being constructed for the entire Center (see Figures 6 and 7). The units of the grid are in hectares (a hectare equals 2.47I acres or Io,००० square meters). The hectare was chosen to facilitate the future establishment of sample plots, since it can easily be broken down into smaller metric units. Once the location of a species is established the reader may check the soil and vegetation maps (Figures 2 to 5 ) for additional details.

The remaining annotation data summarize the vegetation of the collecting sites. The dominant species are listed when these are apparent. Also listed are "associate species" collected or observed at the same location and in the same stratum. Further investigation of the coincidence of these associate species should lead to more reliable correlations among them and with their habitats. Additional details of the vegetation may be obtained from Figures 4 and 5 and from Table I.

The specimen collection numbers of each species follow its annotation. All specimens collected by the writer are preserved in the U. S. National Herbarium, Washington, D.C. Duplicate specimens will be filed at the Chesapeake Bay Center. A few additional specimens collected by Dr. H. David Hammond are kept in the Howard University Herbarium, Washington, D.C. 


\section{Hectare Coordinates of the Chesapeake Bay Center for Field Biology}

These maps (Figures 6, 7) represent a grid system which provides a large number of fixed points for designating sampling stations and recording collections. Each square of the grid is Ioo meters on a side and has an area of Io,००० square meters or I hectare (I hectare $=2.47 \mathrm{I}$ acres). The squares may be easily broken down into smaller metric units. Each of the larger squares (with heavier borders) contains Ioo hectare squares.

The vertical grid lines run north and south at right angles to the baseline, not according to magnetic north. The heavy horizontal line crossing Java Farm is the baseline; trees and brush along this line have been cleared to make a relatively open footpath. Disturbance to the vegetation along the other grid lines has been kept to a minimum.

Although the grid lines on these maps extend beyond the boundaries of the Chesapeake Bay Center, the actual surveying has been confined to the Smithsonian property. In the field the corners of each square are pinpointed either by concrete monuments or by pipes driven into the ground. The monuments are placed two hectares apart, and have brass plates bearing the numbers of the adjoining squares. Where pipes are used, stakes bearing the grid numbers accompany them. No markers have been planted in the estuaries. 
Figure 6.

\section{HECTARE COORDINATES OF JAVA FARM}

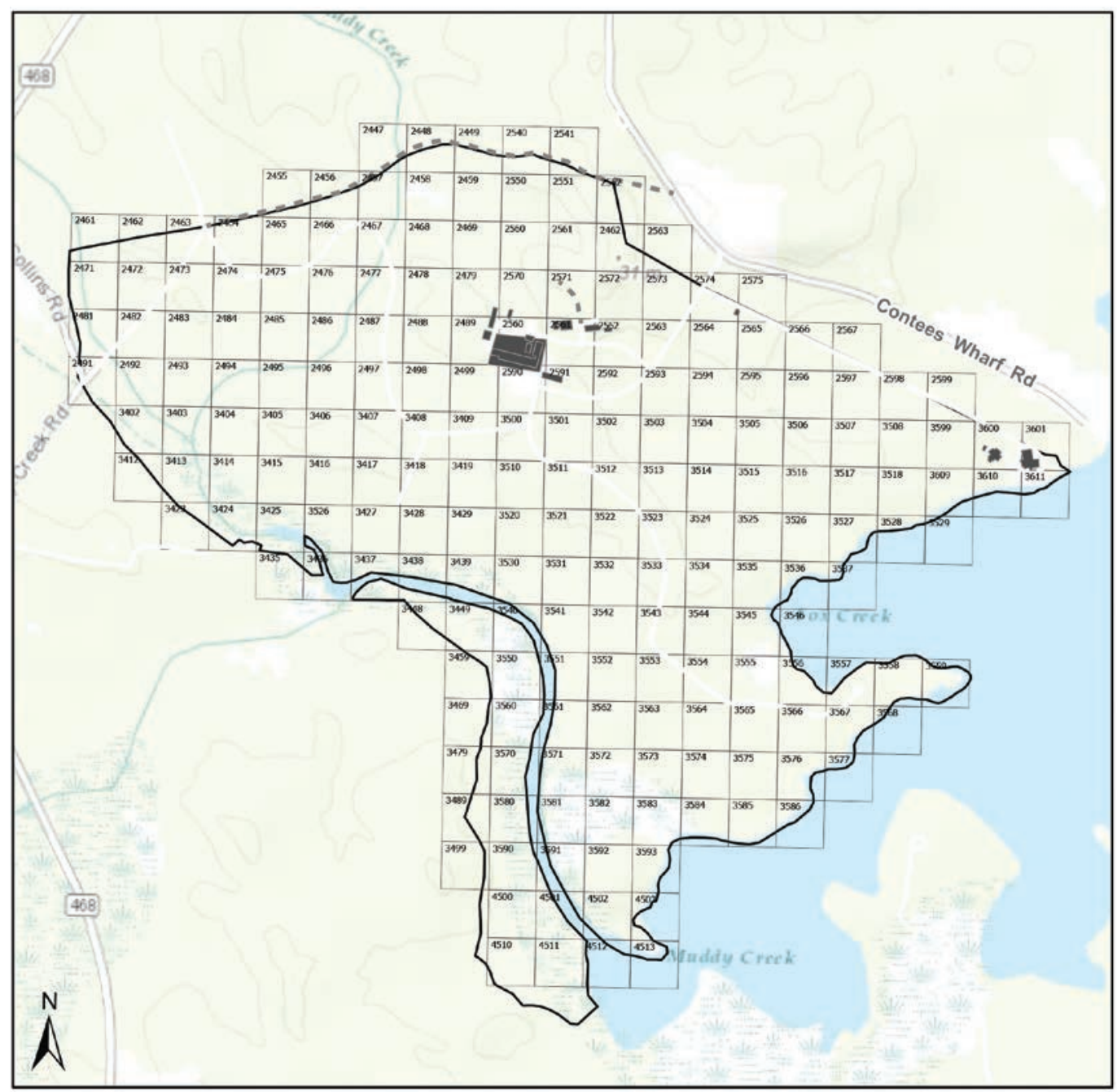


Figure 7.

\section{HECTARE COORDINATES OF IVY NECK AND CORN ISLAND}

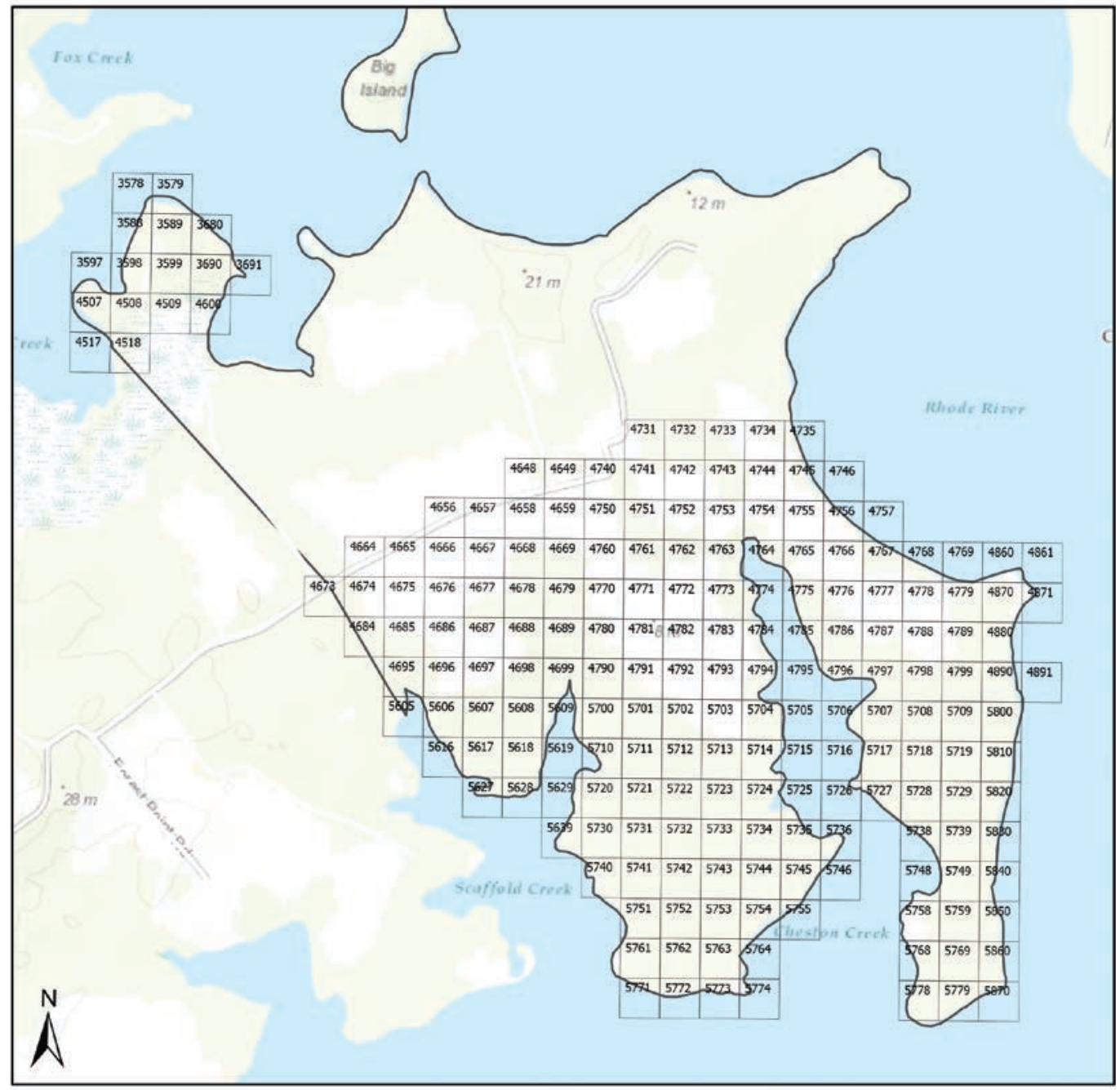




\section{Literature Cited}

Braun, E. Lucy. I950. Deciduous forests of eastern North America. Blakiston Press, Philadelphia. 596 p.

Craven, Avery O. 1926. Soil erosion as a factor in the agricultural history of Virginia and Maryland, I606-I86o. Univ. Illinois Press, Urbana. I79 p.

Dayton, William A. I942. Preliminary treatment of Umbelliferae, Washington-Baltimore Flora. Mimeographed. Distributed by the Conference on District Flora, Smithsonian Institution, Washington, D.C. 34 p.

Fernald, Merritt L. I950. Gray's Manual of Botany, 8th edition. American Book Co., New York. $1632 \mathrm{p}$.

Gilman, Elizabeth M. I957. Grasses of the Tidewater-Piedmont region of northern Virginia and Maryland. Castanea 22: I-I05.

Gleason, Henry A. I952. The new Britton and Brown illustrated flora of the northeastern United States and adjacent Canada. Lancaster Press, Lancaster, Pennsylvania. 3 vol.

Hairston, Nelson G., Frederick E. Smith, and Lawrence B. Slobodkin. I960. Community structure, population control, and competition. American Naturalist 94: 42I-425.

Hermann, Frederick J. I94I. Key to the genus Carex in the Washington-Baltimore area. Mimeographed. Distributed by the Conference on District Flora, Smithsonian Institution, Washington, D.C. 24 p.

- I946. A checklist of plants in the Washington-Baltimore area, 2nd edition. Mimeographed. Issued by the Conference on District Flora, Smithsonian Institution, Washington, D.C. I34 p.

Kelly, J. Reaney. I965. "Tulip Hill," its history and its people. Maryland Hist. Soc. Mag. 6o: $349-403$.

Mack, Frederick K. I962. Ground water supplies for industrial and urban development in Anne Arundel County. Maryland Dept. Geol., Mines, \& Water Res. Bull. 26: 90 p.

Marye, William B. I955. The great Maryland barrens. Maryland Hist. Soc. Mag. 50: II-23, I2O-I42, 234-253.

Middleton, Arthur P. I953. Tobacco Coast, a maritime history of the Chesapeake Bay in the Colonial era. Mariner's Museum, Newport News. 487 p.

O’Neill, Hugh T. I94I. Cyperaceae of the Washington-Baltimore area (exclusive of Carex). Mimeographed. Distributed by the Conference on District Flora, Smithsonian Institution, Washington, D.C. 8 p.

Phillips, S. W., S. O. Perkins, and H. B. Winant. 1928. Soil survey of Anne Arundel County, Maryland. U. S. Dept. Agr., Bur. Chem. and Soils. Ser. 28, no. Io: 29 p., map.

Shelford, Victor E. I963. The ecology of North America. Univ. Illinois Press, Urbana. 6ro p.

Stieber, Michael T. 1967. An annotated checklist of the vascular flora of Anne Arundel County, Maryland. Master of Science thesis, Catholic Univ. of America, Washington, D.C. $70 \mathrm{p}$.

USDA, NRCS. 20I5. The PLANTS Database (http://plants.usda.gov, 23 June 2015). National Plant Data Team, Greensboro, NC 2740I-490i USA.

The Plant List (2013). Version I.I. Published on the Internet; http://www.theplantlist.org/ (accessed ist January). 


\section{Families Represented at the Chesapeake Bay Center for Field Biology}

\begin{tabular}{|c|c|c|c|c|c|c|}
\hline $\begin{array}{l}\text { DIVISION AND } \\
\text { CLASS }\end{array}$ & FAMILY & $\begin{array}{c}\text { NO. } \\
\text { SPECIES }\end{array}$ & PAGE & FAMILY & $\begin{array}{c}\text { NO. } \\
\text { SPECIES }\end{array}$ & PAGE \\
\hline \multicolumn{4}{|l|}{ Pteridophyta: } & \multicolumn{3}{|c|}{ Dicotyledonae (continued): } \\
\hline Articulatae: & Equisetaceae & 1 & 45 & Nymphaeaceae & 1 & 122 \\
\hline Lycopodiinae: & Lycopodiaceae & 1 & 45 & Ranunculaceae & 8 & 122 \\
\hline \multirow[t]{3}{*}{ Filicinae: } & Ophioglossaceae & 2 & 45 & Berberidaceae & 1 & 125 \\
\hline & Osmundaceae & 2 & 46 & Magnoliaceae & 1 & 126 \\
\hline & Polypodiaceae & 10 & 47 & Annonaceae & 1 & 126 \\
\hline \multirow{3}{*}{$\begin{array}{l}\text { Spermatophyta: } \\
\text { Gymnospermae: } \\
\text { Angiospermae: }\end{array}$} & & & & Lauraceae & 2 & 126 \\
\hline & Pinaceae & 3 & 50 & Papaveraceae & 1 & 127 \\
\hline & & & & Cruciferae & 12 & 127 \\
\hline \multirow[t]{17}{*}{ Monocotyledonae: } & Typhaceae & 2 & 51 & Crassulaceae & 1 & 131 \\
\hline & Sparganiaceae & 1 & 52 & Saxifragaceae & 1 & 131 \\
\hline & Zosteraceae & 2 & 52 & Hamamelidaceae & 1 & 131 \\
\hline & Alismataceae & 2 & 52 & Platanaceae & 1 & 131 \\
\hline & Gramineae & 75 & 53 & Rosaceae & 28 & 132 \\
\hline & Cyperaceae & 26 & 77 & Leguminosae & 33 & 140 \\
\hline & Araceae & 2 & 93 & Oxalidaceae & 3 & 149 \\
\hline & Lemnaceae & 2 & 94 & Geraniaceae & 2 & 150 \\
\hline & Commelinaceae & 1 & 95 & Simaroubaceae & 1 & 151 \\
\hline & Pontederiaceae & 1 & 95 & Euphorbiaceae & 4 & 151 \\
\hline & Juncaceae & 8 & 95 & Anacardiaceae & 5 & 152 \\
\hline & Liliaceae & 8 & 97 & Aquifoliaceae & 1 & 153 \\
\hline & Dioscoreaceae & 1 & 100 & Celastraceae & 2 & 153 \\
\hline & Amaryllidaceae & 4 & 100 & Aceraceae & 4 & 154 \\
\hline & Iridaceae & 1 & 101 & Balsaminaceae & 1 & 155 \\
\hline & Orchidaceae & 3 & 101 & Vitaceae & 3 & 155 \\
\hline & & & & Malvaceae & 3 & 156 \\
\hline \multirow[t]{16}{*}{ Dicotyledonae: } & Saururaceae & 1 & 102 & Guttiferae & 4 & 157 \\
\hline & Salicaceae & 3 & 102 & Cistaceae & 1 & 158 \\
\hline & Myricaceae & 1 & 104 & Violaceae & 5 & 158 \\
\hline & Juglandaceae & 4 & 104 & Passifloraceae & 1 & 159 \\
\hline & Betulaceae & 3 & 105 & Lythraceae & 2 & 160 \\
\hline & Fagaceae & 12 & 106 & Nyssaceae & 1 & 160 \\
\hline & Ulmaceae & 4 & 110 & Onagraceae & 7 & 160 \\
\hline & Moraceae & 4 & 111 & Haloragaceae & 1 & 162 \\
\hline & Urticaceae & 1 & 112 & Araliaceae & 2 & 163 \\
\hline & Polygonaceae & 16 & 112 & Umbelliferae & 6 & 163 \\
\hline & Chenopodiaceae & 3 & 117 & Cornaceae & 3 & 166 \\
\hline & Amaranthaceae & 3 & 118 & Pyrolaceae & 2 & 167 \\
\hline & Phytolaccaceae & 1 & 119 & Ericaceae & 9 & 167 \\
\hline & Aizoaceae & 1 & 120 & Primulaceae & 1 & 171 \\
\hline & Portulacaceae & 1 & 120 & Plumbaginaceae & 2 & 171 \\
\hline & Caryophyllaceae & 8 & 120 & Ebenaceae & 1 & 171 \\
\hline
\end{tabular}




\begin{tabular}{|c|c|c|c|c|c|c|}
\hline $\begin{array}{l}\text { DIVISION AND } \\
\text { CLASS }\end{array}$ & FAMILY & $\begin{array}{c}\text { NO. } \\
\text { SPECIES }\end{array}$ & PAGE & FAMILY & $\begin{array}{c}\text { NO. } \\
\text { SPECIES }\end{array}$ & PAGE \\
\hline \multicolumn{7}{|c|}{ Dicotyledonae (continued): } \\
\hline & Oleaceae & 4 & 172 & Bignoniaceae & 1 & 190 \\
\hline & Gentianaceae & 2 & 173 & Orobanchaceae & 1 & 190 \\
\hline & Apocynaceae & 1 & 174 & Phrymaceae & 1 & 190 \\
\hline & Asclepiadaceae & 2 & 174 & Plantaginaceae & 4 & 190 \\
\hline & Convolvulaceae & 5 & 175 & Rubiaceae & 7 & 191 \\
\hline & Boraginaceae & 3 & 176 & Caprifoliaceae & 8 & 193 \\
\hline & Verbenaceae & 4 & 177 & Valerianceae & 2 & 196 \\
\hline & Labiatae & 16 & 179 & Campanulaceae & 3 & 196 \\
\hline & Solanaceae & 6 & 184 & Compositae & 72 & 197 \\
\hline & Scrophulariaceae & 10 & 186 & & & \\
\hline
\end{tabular}

\section{Summary}

DIVISION AND CLASS

NO. FAMILIES

NO. SPECIES

Pteridophyta:

Articulatae:

Lycopodiinae:

Filicinae:

$\begin{array}{rr}1 & 1 \\ 1 & 1 \\ 3 & 14\end{array}$

Spermatophyta:

Gymnospermae:

1

3

Angiospermae:

Monocotyledonae: $\quad 16 \quad 139$

Dicotyledonae: $\quad 76 \quad 387$

Totals:

98

545

13 species in Appendix I.

558 


\section{Adventive and Introduced Species at the Chesapeake Bay Center}

Pteridophyta:

Filicinae:

Osmunda regalis $\mathrm{L}$.

Athyrium filix-femina (L.) Roth

Spermatophyta:

Gymnospermae: None

Angiospermae:

Monocotyledonae:

Gramineae

Anthoxanthum odoratum L.

Arrhenatherum elatius (L.) Mert.

\& Koch

Bromus commutatus Schrad.

inermis Leyss.

japonicus Thunb.

racemosus $\mathrm{L}$.

Cynodon dactylon (L.) Pers.

Dactylis glomerata L.

Digitaria ischaemum (Schreb.) Muhl,

Eleusine indica (L.) Gaertn.

Festuca elatior $\mathrm{L}$.

Holcus lanatus L.

Phleum pratense $\mathrm{L}$.

Phragmites communis Trin.

Poa annua L.

compressa L.

Secale cereale L.

Setaria glauca (L.) Beauv.

faberii Herrm.

Sorghum halepense (L.) Pers.

Triticum aestivum L.

Cyperaceae

Scirpus validus Vahl. var. creber Fern.

Commelinaceae

Commelina communis L.

\author{
Liliaceae \\ Allium vineale $\mathrm{L}$. \\ Asparagus officinalis L. \\ Hemerocallis fulva $\mathrm{L}$. \\ Narcissus poeticus L. \\ pseudo-narcissus \\ Ornithogalum umbellatum L.
}

Dicotyledoneae:

X Populus eugenei Simon-Louis

Ulmus procera Salisb. pumila L.

\section{Moraceae}

Broussonetia papyrifera (L.) Vent.

Madura pomifera (Raf.) Schneid.

Morus alba L.

Polygonum aviculare cespitosum Blume var. longisetum (DeBruyn) Stewart persicaria L.

Rumex acetosella L. crispus L. obtusifolius L. pulcher $\mathrm{L}$.

\section{Chenopodiaceae \\ Chenopodium album L. \\ Amaranthaceae \\ Amaranthus hybridus L. spinosus L.}

Mollugo verticillata $\mathrm{L}$. 


\author{
Caryophyllaceae \\ Cerastium viscosum L. \\ vulgatum $\mathrm{L}$. \\ Dianthus armeria L. \\ Lychnis alba Mill. \\ Silene noctoflora $\mathrm{L}$. \\ Stellaria media (L.) Cyrillo
}

Ranunculaceae

Clematis dioscoreifolia Levi. \& Vaniot

Ranunculus bulbosus L.

\section{Cruciferae}

Arabidopsis thaliana (L.) Heynh.

Barbarea verna (Mill.) Aschers. vulgaris $\mathrm{R}$. Br.

Cardamine hirsuta L.

Draba verna $\mathrm{L}$.

Lepidium campestre (L.) R. Br.

Nasturtium officinale R. Br.

Sisymbrium officinale (L.) Scop.

\section{Crassulaceae}

Sedum sarmentosum Bunge

Rosaceae

Duchesnea indica (Andr.) Focke

Potentilla recta $\mathrm{L}$.

Prunus avium $\mathrm{L}$. persica (L.) Batsch

Pyrus communis L. malus $\mathrm{L}$.

Rosa canina L.

micrantha Sm.

multiflora Thunb.

Rubus phoenicolasius Maxim.

\section{Leguminosae}

Lespedeza cuneata (Dumont) G. Don . stipulacea Maxim.

Medicago lupulina L.

Melilotus alba Desr. officinalis (L.) Lam.
Trifolium arvense $\mathrm{L}$. hybridum L. pratense $\mathrm{L}$. procumbens $L$. repens $L$.

Vicia angustifolia Reichard sativa $\mathrm{L}$. tetrasperma (L.) Moench. villosa Roth

Simaroubaceae

Ailanthus altissima (Mill.) Swingle

Acer platanoides L.

Malvaceae

Abutilon theophrasti Medic.

Guttiferae

Hypericum perforatum L.

Violaceae

Viola kitaibeliana R. \& S. var. rafinesquii (Greene) Fern.

Ludwigia palustris (L.) Ell.

Myriophyllum spicatum L.

$\underline{\text { Haloragaceae }}$

Umbelliferae

Daucus carota L.

Primulaceae

Anagallis arvensis L.

Syringa vulgaris $\mathrm{L}$.

\section{Oleaceae}

Ligustrum vulgare $\mathrm{L}$.

Convolvulaceae

Ipomoea hederacea (L.) Jacq. purpurea (L.) Roth 
Labiatae

Glechoma hederacea L. var. micrantha Moricand

Lamium amplexicaule L. purpureum L.

Mentha spicata L.

Perilla frutescens (L.) Britt.

Solanaceae

Datura stramonium L.

Solanum nigrum L.

sarachoides Sendtner

Scrophulariaceae

Paulownia tomentosa (Thunb.) Steud.

Verbascum blattaria L. thapsus L.

Veronica arvensis $\mathrm{L}$. hederaefolia L.

Plantaginaceae
$\begin{gathered}\text { Plantago lanceolata L. } \\ \text { major L. }\end{gathered}$

Caprifoliaceae

Lonicera japonica Thunb.
Valerianella olitoria (L.) Poll.

Achillea millefolium L.

Anthemis arvensis L.

Arctium minus (Hill) Bernh.

Centaurea cyanis $\mathrm{L}$.

Chondrilla juncea L.

Chrysanthemum leucanthemum $\mathrm{L}$.

Cichorium intybus L.

Cirsium arvense (L.) Scop. vulgare (Savi) Tenore

Galinsoga ciliata (Raf.) Blake

Hieracium scabrum Michx. venosum $\mathrm{L}$.

Lactuca canadensis L. floridana (L.) Gaertn.

Pyrrhopappus carolinianus (Walt.) DC.

Rudbeckia serotina Nutt.

Sonchus asper (L.) Hill

Taraxacum officinale Weber 


\section{Plant Annotations (Taxonomy and Locational Information)}

\section{EQUISETACEAE \\ Equisetum}

Equisetum arvense L
Field Horsetail

(Common Horsetail)

Java Farm. One station: rubble of ruined house at main building area. Hectare 2580. Higman 752.

\section{LYCOPODIACEAE}

Lycopodium

Lycopodium digitatum Dill. ex A. Braun

Fan Clubmoss

(Lycopodium complanatum L. var. flabelliforme Fern.)

(Groundpine)

Java Farm. Moderately abundant on north-and west-facing slopes of ridge between Muddy Creek valley and main building area. Hectares 2477, 2478, 2487. Canopy of mature Pinus virginiana, Fagus grandifolia, Liriodendron tulipifera, Liquidambar styraciflua, etc. Higman 489.

\section{OPHIOGLOSSACEAE \\ Botrychium \\ (Botrichium)}

I. Sterile blade sessile, about midway on the stalk; plant fruiting in spring. (Also, divisions of the sterile blade spatulate and serrate or cleft) . . . . B. virginianum

I. Sterile blade on a petiole half the height of the stalk; plant fruiting in autumn. (Species typically has divisions of the sterile blade cleft into many linear notched teeth; in forma obliquum the divisions are broader and crenate-serrulate to entire) $\ldots \ldots \ldots \ldots \ldots \ldots \ldots \ldots \ldots \ldots \ldots \ldots \ldots \ldots \ldots \ldots \ldots \ldots \ldots \ldots$ dissectum 
Java Farm. Moderately abundant in mature upland forest west of Muddy Creek valley. Canopy of Fagus grandifolia, Carya tomentosa, Quercus alba, Liriodendron tulipifera, etc. Associate species: Botrichium dissectum.

Ivy Neck. Moderately abundant in mature forest of central Scaffold Peninsula. Canopy resembles that at Java Farm but lacks Fagus grandifolia. Same associate species. Higman I24I.

\section{B. dissectum Spreng.}

Cutleaf Grapefern

(Botrychium dissectum Spreng. (typical))

(Grape Fern)

Java Farm. Infrequent in same habitat as B. virginianum above. Also one station at north edge of Phalaris arundinacea meadow and base of south-facing slope (hectare 2469). Higman I55.

\section{B. dissectum Spreng.}

(B. dissectum forma obliquum (Muhl.) Fern. (var. obliquum (Muhl.) Clute)

Java Farm. One station along northern border between Muddy Creek Road gate and Muddy Creek. Associated with typical form above.

Ivy Neck. One station on Cheston Peninsula, in mature stand of Pinus taeda. Hectare 57I9. Higman IIOI, IIO2.

\section{OSMUNDACEAE}

\section{Osmunda}

I. Sterile and fertile fronds bipinnate, the sterile with simple pinnules . . . . . . . $\ldots \ldots \ldots \ldots \ldots \ldots \ldots \ldots \ldots \ldots \ldots \ldots \ldots \ldots \ldots \ldots \ldots \ldots \ldots \ldots \ldots$ regalis

I. Sterile fronds once pinnate, with pinnatifid pinnae; fertile fronds separate, cinnamon-colored, densely wooly. .............. O. cinnamomea

Osmunda regalis L. var. spectabilis (Willd.) A. Gray

Royal Fern (Osmunda regalis L. var. spectabilis (Willd.) Gray) (var. not in Britton \& Brown))

Java Farm. One station: Ravine on east-facing slope between Fox Point Road and Fox Creek estuary. Hectare 3544. Canopy of Pinus virginiana. Associate species: Osmunda cinnamomea. Higman 516.

O. cinnamomea L.

Cinnamon Fern

Java Farm. Principal station on north shore of Hog Island near junction with salt marsh. Hectare 3582. Canopy of Quercus prinus, Q. alba, etc. Second station in Hog Island salt marsh near border of mainland. Hectare 3572. Partial canopy of young 
Quercus falcata, C. stellata. C. phellos. Third station at east-facing slope between Fox Point Road and Fox Creek estuary. Hectare 3544 (same site as O. regalis above). Canopy of Pinus virginiana. Higman I04, 442, 515.

\section{DRYOPTERIDACEAE, THELYPTERIDACEAE, ASPLENIACEAE (POLYPODIACEAE)}

I. Indusium obsolete.

2. Fronds leathery, evergreen, simply pinnatifid. Expected (Polypodium).

2. Fronds herbaceous, deciduous, 2-to 4-times pinnate or pinnatifid.

3. Rhizome short and stout, forming a crown; fronds lanceolate, in vase-like

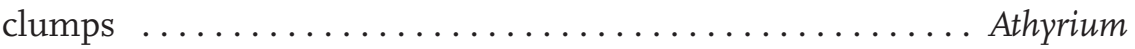

3. Rhizome slender, long and forking; fronds deltoid or broader, solitary or

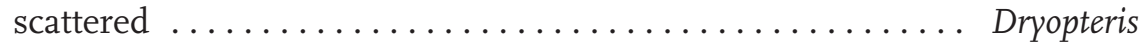

I. Indusium present.

4. Indusium formed by revolute margin of the frond. Expected (Pellaea, Cheilanthes, Dennstaedtia).

4. Indusium not formed by margin of frond, either dorsal or marginal.

5. Indusium borne beneath the sorus, surrounding its base as a saucershaped structure.

6. Fertile and sterile fronds similar, not rigid. Expected (Woodsia, Dennstaedtia).

6 . Fertile and sterile fronds very dissimilar, stiff, the sterile much larger, the fertile erect and rigid.

7. Fronds forming tall vase-like clumps, the sterile and fertile simply pinnate. Expected (Pteretis).

7. Fronds solitary or scattered, the sterile coarsely pinnatifid, the fertile bi-pinnate .......................... Onoclea

5. Indusium spreading above or outside the sorus.

8. Indusium attached at center, orbicular to reniform.

9. Indusium shield-shaped, without a sinus ......... Polystichum

9. Indusium reniform or with deep sinus . .......... Dryopteris

8. Indusium attached at margin, linear or oblong to crescent-shaped. (Also, sori parallel to oblique lateral veins).

Io. Fronds deciduous, pinnate to tripinnate; stipes flat, angled or furrowed $\ldots \ldots \ldots \ldots \ldots \ldots \ldots \ldots \ldots$ Athyrium

Io. Fronds evergreen, pinnate (in species collected); stipes slender, nearly terete ........................ Asplenium

\section{Athyrium}

I. Fronds bi- to tripinnate; sori roundish or reniform; indusium crescent-shaped $\ldots \ldots \ldots \ldots \ldots \ldots \ldots \ldots \ldots \ldots \ldots \ldots \ldots \ldots \ldots \ldots \ldots \ldots \ldots \ldots \ldots \ldots$ filix-femina

I. Fronds simply pinnate or deeply bipinnatifid; sori straight or nearly so. Expected (A. pycnocarpon). 


\section{Athyrium filix-femina}

I. Rhizome compact, covered with persistent old stipe-bases; frond widest near middle; indusia toothed, if ciliate the hairs not gland-tipped. (Also, fertile fronds more leathery and contracted than the sterile; mature sori confluent) $\ldots \ldots \ldots \ldots \ldots \ldots \ldots \ldots \ldots \ldots \ldots \ldots \ldots \ldots \ldots \ldots$. michauxii

I. Rhizome horizontally creeping, with few or no stipe-bases; frond widest toward base; indusia ciliate, the cilia gland-tipped ........... var. asplenioides

\section{Athyrium filix-femina (L.) Roth ssp. angustum}

Subarctic Ladyfern (Willd.) R.T. Clausen

(Lady Fern)

(A. filix-femina (L.) Roth var. michauxii (Spreng.)

Farw. (var. michauxii Mett.))

Ivy Neck. One station: mature stand of Pinus taeda on Cheston Peninsula. Hectare 57I9. Higman iı03.

A. filix-femina (L.) Roth ssp. angustum (Willd.) R.T. Clausen Subarctic Ladyfern (A. filix-femina var. michauxii form rubellum Farw.)

(A. angustum var. rubellum Butters)

Java Farm. One station: along Muddy Creek entrance road, at culvert of small tributary of Muddy Creek. Hectare 2497. Canopy of Platanus occidentalis. Hig$\operatorname{man} 480$.

\section{A. filix-femina (L.) Roth ssp. asplenioides (Michx.) Hultén \\ Asplenium Ladyfern} (A. filix-femina var. asplenioides (Michx.) Farw.)

(A. asplenioides A. Eaton)

Java Farm. One station: floor of tributary valley of Muddy Creek, slightly upstream from Hog Island salt marsh. Hectare 3530. Canopy of Fagus grandifolia, Quercus alba, Liriodendron tulipifera, Liquidambar styraciflua. Higman 456.

\section{Thelypteris, Dryopteris}

I. Rhizome slender, forking, long; fronds thin, annual, scattered; stipe slender, naked or with scales $\mathrm{I}-6 \mathrm{~mm}$ long.

2. Fronds long-lanceolate, with I7 to 46 pairs of pinnae, the lowest pinnae shorter than to equaling the middle ones.

3. Lateral veins of segments of sterile fronds forking; indusia glabrous or

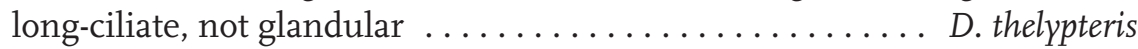

3. Lateral veins of segments of sterile fronds simple; indusia finely glandularciliate. (Also, 23 to 46 pairs of pinnae) ........... D. noveboracensis

2. Fronds triangular to triangular-ovate, simple or with 8-30 pairs of pinnae, the lowest pinnae longest. Expected (D. hexagonoptera), See Appendix I, p. 225. 
I. Rhizome stout, short; fronds firm to evergreen, forming terminal crowns; stipe stout, with abundant basal scales $0.5-3.5 \mathrm{~cm}$ long. (Also, lowest pinnae mostly over $2 \mathrm{~cm}$ long and I $\mathrm{cm}$ wide; indusia not overlapping).

4. Frond tripinnate or tripinnatifid, firm, evergreen; teeth of frond segments bristle-tipped ............................... dilata

4. Frond bipinnate or bipinnatifid, firm to leathery, deciduous; teeth of segments not bristle-tipped. (Also, sori not marginal; fertile fronds much taller and narrower than the sterile) $\ldots \ldots \ldots \ldots \ldots \ldots \ldots \ldots$. cristata

Thelypteris palustris Schott var. pubescens (G. Lawson) Fernald Eastern Marsh Fern (Dryopteris thelypteris (L.) Gray var. pubescens (Lawson) nakai) Marsh Fern (Thelypteris palustris Schott. var. pubescens (Lawson) Fern.)

Java Farm. Infrequent. Principal station in Typha latifolia salt marsh at upstream end of tidal portion of Muddy Creek. Hectare 3403. Also in ravines on east-facing slope between Fox Point Road and Fox Creek estuary. Hectares 3543, 3544. Canopy of Pinus virginiana. Higman 286, 524.

T. noveboracensis (L.) Nieuwl.

New York Fern

(D. noveboracensis (L.) Gray)

(T. noveboracensis (L.) Nieuwl)

Java Farm. One station: source of Fox Creek drainage at north boundary, west of new entrance gate. Hectare 2573. Canopy of young Ulmus americana, Liquidambar styraciflua, and Juglans nigra. Higman 3 I7.

D. expansa (C. Presl) Fraser-Jenkins \& Jermy

Spreading Woodfern

(D. dilatata (Hoffmann) Gray)

(Florist's Fern)

(D. spinulosa (O. F. Muell) Watt, var.)

Java Farm. One station: near western apex of triangular area west of old Muddy Creek Road. Hectare 2470. Level ground adjacent marsh; canopy of mature Fagus grandifolia. A rare species in this region. Higman 251.

Dryopteris cristata (L.) A. Gray

Crested Woodfern

(D. cristata (L.) Gray)

Java Farm. One station: bottom of Muddy Creek valley. Canopy of mature Fraxinus americana, Platanus occidentalis. Higman 74.

Onoclea sensibilis L.

Onoclea

Java Farm. Two stations: bank of Muddy Creek upstream from culvert of old entrance road. Canopy of Platanus occidentalis, Fagus grandifolia, Fraxinus americana. Also found along deep, narrow ditch of Fox Creek drainage. Higman I82, 325. 


\section{Polystichum}

Polystichum acrostichoides (Michx.) Schott.

Christmas Fern

Java Farm. Infrequent to moderately abundant on shaded slopes. Found along trail at north border between Muddy Creek and old entrance gate, in forest overlooking Rhode River and north side of Fox Creek salt marsh, and on east-facing slope west of main buildings. Grows under a variety of hardwood canopies. Higman I60, I6I, $237,376,497$.

\section{Asplenium}

Asplenium platyneuron (L.) Britton, Sterns \& Poggenb. (Asplenium platyneuron (L.) Oakes)

Ebony Spleenwort

Java Farm. Infrequent. Found along trail at north border between Muddy Creek and old entrance gate, in old fields, and on bank overlooking salt marsh at northeast corner of Farm.

Ivy Neck. One station on bank overlooking halophytic area at Sand Point, on Cheston Peninsula. Hectare 4860. Higman I43, I62, I64, 209, 218, 470, 875.

\section{CUPRESSACEAE, PINACEAE}

I. Leaves opposite or in 3's, scale-like; fruit berry-like, blue and glaucous . . . . . .

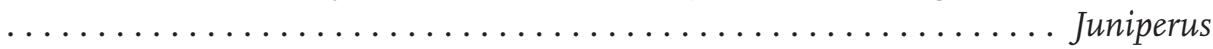

I. Leaves sheathed in fascicles; fruit a woody cone $\ldots \ldots \ldots \ldots \ldots \ldots$ Pinus

\section{Juniperus virginiana L. var. virginiana}

Juniperus

(Juniperus virginiana L. var. crebra Fern. \& Grisc.)

Eastern Redcedar

(Redcedar)

Java Farm and Ivy Neck. Moderately abundant along roads and fences, in old fields, and in a variety of hardwood forest types. Infrequent in dry, halophytic areas and salt marshes. Higman II2, 259, II30.

\section{Pinus}

I. Needles I4-25 cm long; cone scales with stout spine $\ldots \ldots \ldots \ldots \ldots$. taeda

I. Needles usually shorter than I2 cm; cone scales with awl-shaped spine.

2. Needles in 3's. Expected (P. rigida, P. serotina).

2. Needles in 2's.

3. Twigs glaucous; needles 7-I2 cm long; spine of cone scale I mm long, weak. Expected (P. echinata).

3. Twigs brown, not glaucous; needles $4-8 \mathrm{~cm}$ long; spine of cone scale 2-3 mm long, firm, straight or recurved .......... P. virginiana 
Java Farm. Infrequent; mature stand on south-facing slope of ridge, west of main buildings and below water tank. Scattered through canopy of mature Pinus virginiana, Fagus grandifolia, and Quercus alba on adjacent west-facing slope. Young individuals scattered in old fields.

Ivy Neck. Mature, planted stand on lower Cheston Peninsula. Higman 4I, 4I5, 508, 574,649 .

P. virginiana Mill.

Virginia Pine

(Scrub Pine)

Java Farm and Ivy Neck. Frequently dominates understory of Quercus prinus forest; infrequent to moderately abundant in old fields, there associated with Robinia pseudoacacia, Liquidambar styraciflua, Acer rubrum, etc.

Java Farm. Dominant or co-dominant on west-facing slope overlooking old entrance road, and along Fox Point Road near base of Fox Point Peninsula. Higman 265.

\section{TYPHACEAE}

\section{Typha}

I. Staminate and pistillate parts of the spike contiguous; pistillate part I.5 to $3.5 \mathrm{~cm}$ in diameter . . . . . latifolia

I. Staminate and pistillate parts of the spike separated; pistillate part 6 to $\mathrm{I} 2 \mathrm{~mm}$

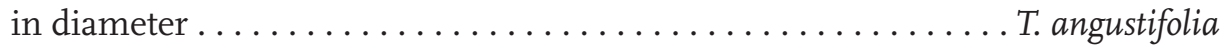

Typha latifolia L.

Broadleaf Cattail

(Common Cattail)

Java Farm and Ivy Neck. Abundant or moderately abundant in salt marshes near heads of estuaries and at the landward margins of more saline marshes. Associate species: Dryopteris thelypteris (co-dominant in one marsh near head of tidal portion of Muddy Creek), Scirpus americanus (co-dominant in marsh at head of Cheston Creek estuary), Hibiscus palustris, Kosteletzkya virginica, Panicum virgatum (infrequent at landward margins of other salt marshes). Higman 90.

T. angustifolia L.

Narrowleaf Cattail

(Narrow-leaved Cattail)

Java Farm and Ivy Neck. Infrequent to moderately abundant near landward margins of salt marshes. Associate species: Typha latifolia. Higman 2II, 533. 


\section{SPARGANIACEAE}

Sparganium

Sparganium eurycarpum Engelm.

Broadfruit bur-reed

(Burreed)

Ivy Neck. One station: large clump on dried mud bottom of cut-off pond on Cheston Peninsula. Hectare 4880. Associate species: Rumex verticillatus, Sambucus canadensis, Sium suave. Higman 944.

\section{POTAMOGETONACEAE, ZANNICHELLIACEAE \\ (ZOSTERACEAE)}

I. Flowers in spikes or on a long axis.

2. Spike more than 2-flowered, raised above the stipule in anthesis; carpels in

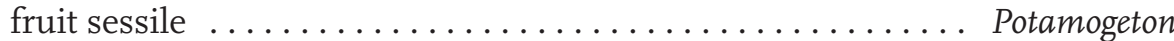

2. Spike 2-flowered, enclosed in the sheathing leaf base in anthesis; carpels in fruit raised on slender stalks. Expected (Ruppia).

I. Flowers axillary $\ldots \ldots \ldots \ldots \ldots \ldots \ldots \ldots \ldots \ldots \ldots \ldots \ldots \ldots$ Zannichellia

\section{Potamogeton}

Potamogeton perfoliatus L.

Claspingleaf Pondweed

(Potamogeton perfoliatus L. var. bupleuroides (Fern.) Farw.)

(Pondweed)

Java Farm and Ivy Neck. Abundant in estuaries. Associate species: Myriophyllum spicatum. Higman 272, I009.

\section{Zannichellia}

Zannichellia palustris L.

Horned Pondweed

Ivy Neck. One station: cut-off pond on Cheston Peninsula. Higman II54.

\section{ALISMATACEAE}

I. Carpels in a single ring, strongly flattened; style on the ventral margin of the carpel, below the tip; flowers all perfect; stamens $6 \ldots \ldots \ldots$. . . . Alisma

I. Carpels in dense heads; style apical; upper flowers staminate (or all either staminate or pistillate); stamens numerous . . . . . . . . . . Sagittaria 
Ivy Neck. One station: shore of small cut-off pond on Cheston Peninsula, south of Sand Point. Hectare 5800. Associate species: Rumex verticillatus, Cephalanthus occidentalis, Echinochloa walteri, Galinsoga ciliata. Higman 946.

Sagittaria latifolia Willd.

\section{Sagittaria}

(Sagittaria latifolia Willd. var. pubescens (Muhl.) J. C. Sm.)

Broadleaf Arrowhead

Duckpotato

Java Farm. One station: dry, sandy bed of north fork of Muddy Creek, upstream from culvert of old entrance road, near barrier of piling (collected during drought). Hectare 2467. Canopy of Platanus occidentalis, Fagus grandifolia, and Liriodendron tulipifera. Under-story chiefly Lindera benzoin. Associate species: Impatiens capensis, Polygonum arifolium. Also observed in the freshwater part of Fox Creek marsh. Hectare 3526. Higman I056.

\section{POACEAE \\ (GRAMINEAE)}

Key to Tribes

I. Spikelets I-to-many-flowered; articulation above the glumes except in Oryzeae.

2. Plants woody; culms perennial .............. Bambuseae (Expected)

2. Plants herbaceous; culms annual.

3. Spikelets 3 -flowered, with 2 rudimentary florets below the perfect one... Phalarideae (p. 54)

3. Spikelets I- to -several-flowered, with no rudimentary florets below the perfect one (but sometimes above it).

4. Spikelets unisexual; plants monoecious.

5. Pistillate spikelets embedded in joints of the rachis... Tripsaceae (p. 54)

5. Pistillate spikelets not embedded in the joints of the rachis .......

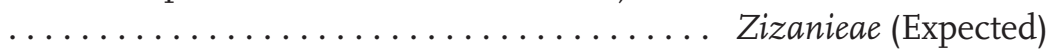

4. Spikelets perfect or, if unisexual, the plants dioecious.

6 . Glumes minute or wanting; articulation below them .......... ................................. Oryzeae (p. 55)

6. Glumes, or at least one of them, well-developed; articulation above them.

7. Spikelets sessile; rachis continuous except in Hordeum.

8. Spikelets on opposite sides of the rachis; spikes terminal, solitary ........................Hordeae (p. 55)

8. Spikelets on one side of the rachis; spikes digitate or racemose Chlorideae (p. 57) 
7. Spikelets pedicellate, in open, contracted, or spikelike panicles, rarely in racemes. (Spikelets subsessile in Phleum).

9. Spikelets usually I-flowered ............ Agrostideae (p. 59)

9. Spikelets 2-to several-flowered.

IO. Glumes as long as the lowest floret, usually as long as the spikelets; lemmas awned from the back (awnless in Sphenopholis, awned between the teeth of a bifid apex in Danthonia) ..................... Aveneae (p. 6I)

Io. Glumes, or at least one of them, shorter than the first floret; lemmas awnless or awned from the tip, or from the back just below the tip, or from between the teeth of a bifid apex ....................... Festuceae (p. 63)

I. Spikelets with I perfect terminal floret and one imperfect or sterile lower floret; articulation below the glumes.

II. Spikelets paired, one sessile and perfect, the other pediceled and staminate, sterile, or wanting .................... Andropogoneae (p. 69)

II. Spikelets single (or in rows on one side of a continuous rachis in Digitaria and Paspalum) .......................... Paniceae (p. 70)

\section{Phalarideae}

I. Lower florets reduced to small, awnless, scale-like lemmas, smaller than the fertile florets; glumes equal, sometimes winged on the keel ........ Phalaris

I. Lower florets consisting of awned, hairy, sterile lemmas exceeding the fertile floret; glumes very unequal $\ldots \ldots \ldots \ldots \ldots \ldots$ Anthoxanthum

Phalaris arundinacea $\mathrm{L}$.

\section{Phalaris}

Reed Canarygrass

(Reed Canary-grass)

Java Farm. Abundant in somewhat marshy 5 -acre meadow north of main buildings. Infrequent on roadsides near main buildings. Higman I49, 772, 779, 826, 894 .

Anthoxanthum

Anthoxanthum odoratum L.

Sweet Vernalgrass

(Sweet Vernal Grass)

Ivy Neck. One station. Miss A. Colhoun's garden. Higman II55.

Tripsacum

(Tripsaceae)

Tripsacum dactyloides L.
Eastern Gamagrass

(Gamagrass)

Java Farm. Infrequent, along roads and in scattered clumps in old fields. Higman 25. 


\section{Oryzeae \\ Leersia}

I. Lower panicle branches fascicled; spikelets about $5 \mathrm{~mm}$ long; leaf sheaths and blades very harshly scabrous; rhizomes long and slender . . . . . . L. oryzoides

I. Lower panicle branches not fascicled; spikelets about $3 \mathrm{~mm}$ long; leaf sheaths and blades scabrous but not very harsh, not ciliate; rhizomes short, thick, and

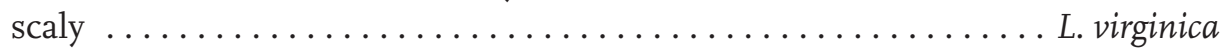

Leersia oryzoides (L.) Sw.

(Leersia oryzoides (L.) Wx.)

Rice Cutgrass

(Rice-Cutgrass)

Java Farm. One station: in freshwater part of Fox Creek marsh. Hectare 3516. Associate species: Rosa palustris, Aster puniceus, Impatiens capensis. Higman 246.

L. virginica Willd.

Whitegrass

Java Farm. Infrequent, in woods near bank of Muddy Creek upstream from entrance road culvert; also along Fox Creek drainage; also one station in old field south of old entrance road. Associate species: Onoclea sensibilis, Epilobium coloratum, Glyceria striata, Elephantopus carolinianus. Higman I4I, I88, 326.

I. Spikelets solitary at each node of rachis.

2. Spikelets placed edgewise to the rachis .............. Lolium

2. Spikelets placed flatwise to the rachis.

3. Annuals, cultivated.

4. Glumes ovate, 3 -nerved . . . . . . . . . . . . . . . Triticum

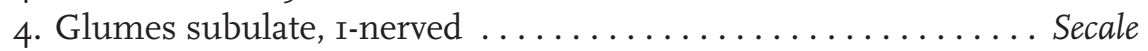

3. Perennials, not cultivated; plants with creeping rhizomes ...... Agropyron

I. Spikelets usually more than I at each node of the rachis.

5. Spikelets I-flowered, 3 at each node, the middle one sessile and perfect, the lateral pair usually pediceled, often reduced to awns and simulating an

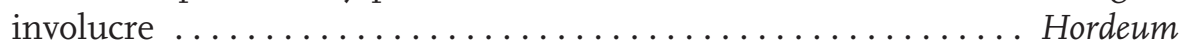

5. Spikelets 2-to 6-flowered, all alike, usually 2 or more at each node.

6. Spikes dense; glumes as long as the lemmas, simulating an involucre; spikelets ascending or slightly spreading ............ Elymus

6. Spikes loose; glumes reduced to minute awn; spikelets widely spreading Hystrix 
Java Farm. One station: along north boundary fence, adjacent neighboring cornfield. Associate species: Clematis virginiana. Amaranthus hybridus, Ambrosia artemisiifolia.

Ivy Neck. One station: waste ground around old house, near entrance road, above drainage of east fork of Scaffold Creek. Hectare 4658. Associate species: Elymus villosus, Plantago aristata. Higman $769,863$.

\section{Triticum}

Triticum aestivum L.

Common Wheat

(Wheat)

Ivy Neck. One station: path to abandoned wheat field on upper Scaffold Peninsula. Hectare 4740. Escaped from cultivation. Associate species: Polygonum avicular, Polygonum pensylvanicum, Diodia teres, Cyperus strigosus. Higman 684.

\section{Secale}

Secale cereale L.

\section{Cereal Rye}

Ivy Neck. One station: abandoned wheat field on upper Scaffold Peninsula. Hectare 476I. Associate species: Trifolium pratense, Trifolium hybridum. Higman 747.

\section{Elymus repens (L.) Gould}

\section{Elymus}

\section{(Agropyron)}

(Agropyron repens (L.) Beauv.)

Quackgrass

(Quickgrass)

Java Farm. Infrequent: grassy field south of main buildings. Associate species: Agrostis alba, Cirsium discolor, Poa pratensis, Asclepias syriaca. Also one station beside new entrance road. Higman 829, 950.

\section{Hordeum}

Hordeum pusillum Nutt.

Little Barley

Ivy Neck. One station: unpaved trail between cornfields on upper Scaffold Peninsula. Hectare 477I. Associate species: Anthemis arvensis, Plantago virginica, Lepidium virginicum, Rumex acetosella. Higman 697.

\section{Elymus}

I. Glumes narrow, bristle-like; glumes and lemmas hirsute . . . . . . . E. villosus

I. Glumes broadened near the base; glumes and lemmas glabrous or nearly so. 
2. Glumes about I mm wide at the middle, the bases not bowed out; palea much shorter than the lemma. Expected (E. riparius).

2. Glumes I.5-2 $\mathrm{mm}$ wide at the middle, the bases bowed out; palea about equal to the lemma ............................. virginicus

Elymus villosus Muhl. ex Willd.

Hairy Wildrye

(Elymus villosus Muhl.)

(Wild Rye)

Java Farm and Ivy Neck. Infrequent to moderately abundant; seems to prefer moderately moist, moderately shaded sites like borders of forest. No consistent associate species. One station at Java Farm: summit of ridge northeast of main buildings, under canopy of Juglans nigra. Three stations at Ivy Neck, near streams or estuaries. Liquidambar styraciflua canopy. Higman 831, 850, 865, 871.

E. virginicus L.

Virginia Wildrye

(Terrell Grass)

Java Farm and Ivy Neck. Infrequent: dry, sandy sites near salt marshes. Two stations at Java Farm: small salt marsh on Rhode River above pier. Associate species: Asplenium platyneuron, Triodia flava, Rhus typhina, Celastrus scandens. One station at Ivy Neck: bank overlooking dry halophytic area at Sand Point, Cheston Peninsula. Hectare 4860. Higman 225, 227, IIO8.

\begin{abstract}
Elymus
(Hystrix)

Elymus hystrix L. var. hystrix

(Hystrix patula Moench)

Eastern Bottlebrush Grass

(Bottle-brush Grass)

Ivy Neck. One station: side of low bank at southwest border of Sand Point marsh. Hectare 4870 . Junction of sand and mineral soil; shaded by forest. Associate species: Elymus villosus. E. virginicus. Higman 940.
\end{abstract}

\title{
Chlorideae
}

I. Spikelets with more than I perfect floret.

2. Spikes slender, numerous, racemose; spikelets short-pediceled. Expected (Leptochloa).

2. Spikes stout, few, digitate; spikelets sessile.............. Eleusine

I. Spikelets with only I perfect floret, sometimes additional imperfect florets above.

3. Spikelets without additional imperfect florets.

4. Spikes racemose; culms erect; spikelets articulate below the glumes; salt marsh habitat ........................... Spartina

4. Spikes digitate; culms creeping by rhizomes or stolons; spikelets articulate above the glumes; terrestrial habitat .................. Cynodon

3. Spikelets with I or more imperfect florets above the fertile one. Expected (Gymnopogon). 
Infrequent, on unshaded waste ground. Java Farm. One station: rubbish pile at main buildings. Hectare 2580. Associate species: Datura stramonium, Amaranthus spinosus, Solanum nigrum.

Ivy Neck. One station: near artificial pond at source of Cheston Creek drainage. Hectare 4722. Associate species: Datura stramonium, Polygonum persicaria, Abutilon theophrasti, Potentilla norvegica. Higman 499, Iо०I.

\section{Spartina}

I. Plants robust, usually more than I $\mathrm{m}$ tall; keel of glumes and lemmas hispidscabrous or ciliate; blades flat (involute after drying), more than $5 \mathrm{~mm}$ wide; spikelets more than I cm long.

2. First glume nearly as long as the floret; second glume with an awn up to $7 \mathrm{~mm}$ long. Expected (S. pectinata).

2. First glume much shorter than the floret; second glume awnless, acute or mucronate.

3. Margins of blades very harsh and scabrous; glumes strongly hispidscabrous on the keel ................... cynosuroides

3. Margins of blades glabrous or minutely scabrous; glumes soft-hispidulous or ciliate on the keel ....................... alternifolia

I. Plants rather slender, usually less than I $\mathrm{m}$ tall; keels of glumes scabrous; blades involute, less than $5 \mathrm{~mm}$ wide $\ldots \ldots \ldots \ldots \ldots \ldots \ldots \ldots \ldots \ldots \ldots \ldots \ldots$. patens

Spartina cynosuroides (L.) Roth.

Big Cordgrass

(Salt Reedgrass)

Java Farm and Ivy Neck. Moderately abundant in salt marshes on Rhode River and lower estuaries, on seaward side. Associate species: Aster subulatus, Baccharis halimifolia, Scirpus robustus, Atriplex patula. Higman 59.

S. alterniflora Loisel.

(S. alternifolia Loisel.)
Smooth Cordgrass

(Salt-water Cordgrass)

Java Farm and Ivy Neck. Moderately abundant in salt marshes on Rhode River and lower estuaries, and in dry, halophytic areas such as Sand Point. Associate species: Baccharis halimifolia, Distichlis spicata, Cakile edentula. Higman II5, 285. 
Java Farm and Ivy Neck. Moderately abundant in drier, more inland parts of salt marshes and halophytic sandy areas. Forms dense carpets. Associate species: Distichlis spicata. Panicum virgatum, Iva frutescens, Acnida cannabina. Higman 270.

Cynodon dactylon (L.) Pers.

$$
\text { Cynodon }
$$

Java Farm. One station: parking area at main buildings, in cracked pavement. Hectare 2580. Higman 823 .

\section{Agrostideae}

I. Panicle dense, spikelike. (Also, glumes as long as the lemma; articulation above the glumes.)

2. Glumes glabrous, awnless; spikelets II-I4 mm long......... Ammophila

2. Glumes with spreading trichomes, abruptly awned; spikelets not more than

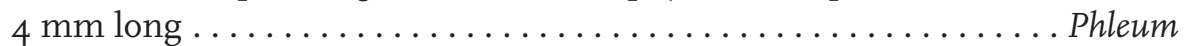

I. Panicle somewhat open, not spikelike. (Also, lemma usually awned except in Cinna and some species of Agrostis).

3. Fruit hardened; callus sharp-pointed. Expected (Aristida).

3. Fruit thin; callus not sharp-pointed.

4. Glumes as long as the lemma or longer.

5. Callus of lemma bearded. Expected (Calamagrostis).

5. Callus of lemma not bearded.

6. Lemma minutely awned from just below the apex; spikelets falling

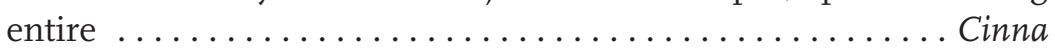

6. Lemma awnless or awned from the back or near the base; glumes

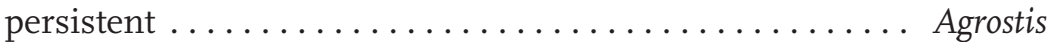

4. Glumes usually shorter than the lemma.

7. Rachilla not prolonged as a bristle behind the palea; grain enclosed in the lemma and palea at maturity. (Also, plant with slender rhizome unless first glume is obsolete) . . . . . . . . . . . Muhlenbergia

7. Rachilla prolonged behind the palea as a bristle; or if not, the grain falling from the lemma and palea at maturity. Expected (Brachyelytrum).

\section{Ammophila}

Ammophila arenaria (L.) Link

European Beachgrass

(Sand-reed)

Ivy Neck. One station: beach on south shore of Sand Point. Hectare 486r. Higman II53. 


\title{
Phleum
}

Phleum pratense L.

Timothy

Ivy Neck. Infrequent in abandoned grassy field on Scaffold Peninsula. Hectare 476I. Associate species: Festuca elatior, Polygonum aviculare, Hypericum perforatum. Higman 8Io.

\section{Cinna arundinacea L.}

\section{Cinna}

\author{
Sweet Woodreed
}

(Wood Reedgrass)

Java Farm. Infrequent in Muddy Creek valley; canopy of Fraxinus americana, Platanus occidentalis; understory of Lindera benzoin. Also one station at forest border of old field south of old entrance road; canopy of Fagus grandifolia and Quercus velutina. Higman 66, 85, I34.

\section{Agrostis}

I. Palea much smaller than the lemma or wanting, nerveless. (Also lemma awnless; plants perennial).

2. Panicle diffuse, its long branches forking at or above the middle. Expected (A. hyemalis).

2. Panicle open but not diffuse, its branches forking at or below the middle.

3. Spikelets mostly $2-2.5 \mathrm{~mm}$ long ............... perennans

3. Spikelets mostly $2.5-3.5 \mathrm{~mm}$ long, crowded near ends of panicle branches. Expected (A. altissima)

I. Palea at least half as long as lemma, 2-nerved. (Also, ligule 2-6 mm long; panicle branches spikelet-bearing to base.)

4. Panicle open; long stolons absent.

5. Culms erect, with strong creeping rhizomes ............. A. alba

5. Culms decumbent; rhizomes wanting. Expected (A. stolonifera).

4. Panicle narrow; long stolons usually present ............ palustris

Agrostis perennans (Walter) Tuck.

Upland Bentgrass

(Agrostis perennans (Walt.) Tuckerm.)

Java Farm. Infrequent, along Fox Creek drainage and in large field adjacent road to pier. Higman 324, 334 .

\section{A. stolonifera L.}

Redtop

(A. alba L.)

(A. stolonifera L.)

Java Farm and Ivy Neck. Infrequent in grassy abandoned fields. Associate species at Java Farm: Agropyron repens, Allium vineale, Cirsium discolor, Asclepias syriaca. Associates at Ivy Neck: Eleocharis intermedia, Carex scoparia, Agropyron repens. Higman 858 , 951. 


\section{(A. palustris Huds.)}

(A. stolonifera L.)

Java Farm. One station: bank of tidal portion of Muddy Creek, near mouth of tributary stream. Hectare 3437. Associate species: Brasenia schreberi, Carex lurida, Polygonum punctatum. Higman 989.

Muhlenbergia schreberi J.F. Gmel.

Muhlenbergia

(Muhlenbergia schreberi Gmel.)

Nimblewill

Java Farm. Infrequent, along Fox Creek drainage and vicinity. Associate species: Perilla frutescens, Duchesnea indica, Aster lateriflorus, Geum canadense. Higman 309, 3I8, 348 .

\section{Aveneae}

I. Florets 2, one perfect, the other staminate.

2. Lower floret staminate, with a long, bent, twisted awn; upper floret perfect

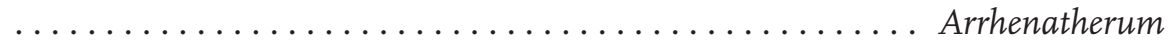

2. Lower floret perfect, awnless; upper floret staminate, with a short, hooked

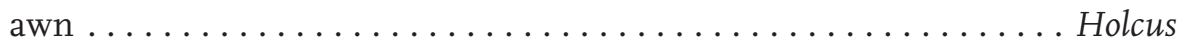

I. Florets 2 or more, all alike except the reduced upper ones.

3. Lemmas bifid, awned from between the lobes; florets several .... Danthonia

3. Lemmas awnless, mucronate, or awned from the back; florets usually 2 or 3 (up to 6 in Avena).

4. Glumes more than I cm long. Expected (Avena).

4. Glumes less than I cm long.

5. Glumes shorter than the florets; lemmas awnless or mucronate.

6. Glumes unequal in shape, the second obovate and much broader than the first. (Also, plant 3-Io mm high in species collected) ..... $\ldots \ldots \ldots \ldots \ldots \ldots \ldots \ldots \ldots \ldots$ Sphenopholis

6. Glumes similar in shape, unequal in size. (Also, plant 3-6 dm high). Expected (Koeleria).

5. Glumes as long as or longer than the florets; lemmas awned from the back.

7. Lemmas keeled, awned from near the apex; awn bent and twisted. Expected (Trisetum).

7. Lemmas rounded on the back, awned from below the middle.

8. Rachilla extending as a bristle behind the upper floret; plants perennial .................... Deschampsia

8. Rachilla not extending; plants delicate annuals. Expected (Aira). 
Java Farm. Infrequent along roads near main building area, and in old fields. Associate species: Triosteum perfoliatum, Galium circaezans, Houstonia purpurea, Lychnis alba. Higman 66I, 716, 763, 778.

\section{Holcus}

Holcus lanatus L.

Common Velvetgrass

(Velvet-grass)

Java Farm. One station: road to springhouse. Hectare 34I7. Associate species: Melilotus alba, Taraxacum officinale, Valerianella olitoria, Duchesnea indica, Rhus radicans. Higman 800.

\section{Danthonia}

Danthonia spicata (L.) P. Beauv. ex Roem. \& Schult.

Poverty Oatgrass

(Danthonia spicata (L.) Beauv.)

(Poverty-grass, White Oatgrass)

Java Farm. One station near marshy west end of Phalaris arundinacea meadow, north of main buildings. Hectare 2467. Associate species: Glyceria striata, Lindera benzoin, Allium vineale, Carex Wanda.

Ivy Neck. Infrequent along banks of upper Cheston Creek estuary. Canopy of Quercus velutina, Prunus serotina, Liquidambar styraciflua, Acer rubrum. Associate species: Hieracium venosum, Amelanchier intermedia, Elymus villosus. Higman 787 , 843,872 .

\section{Sphenopholis}

Sphenopholis nitida (Biehler) Scribn.

Shiny Wedgescale (Sphenopholis nitida (Bieler) Scribn.)

Java Farm. One station: shoulder of old Muddy Creek Road, near entrance gate. Hectare 2473. Higman 643.

$\begin{array}{rr}\text { Deschampsia flexuosa (L.) Trin. } & \text { Deschampsia } \\ \text { Wavy Hairgrass } \\ \text { (Common Hairgrass) }\end{array}$

$\begin{array}{rr}\text { Deschampsia flexuosa (L.) Trin. } & \text { Deschampsia } \\ \text { Wavy Hairgrass } \\ \text { (Common Hairgrass) }\end{array}$

Java Farm. Infrequent on Fox Point Road and moderately abundant at Fox Point under Quercus prinus canopy. Associate species: Rhododendron viscosum, Gaylussacia baccata, Kalmia latifolia, Juncus tenuis, Baptisia tinctoria. Higman 435, 645, 802. 


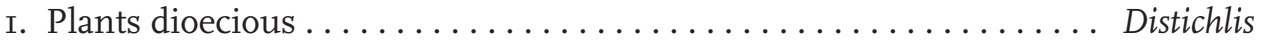

I. Plants not dioecious; flowers usually perfect.

2. Plants more than $\mathrm{I} .5 \mathrm{~m}$ tall; salt marsh habitat ........... Phragmites

2. Plants not more than I. $5 \mathrm{~m}$ tall, usually shorter; terrestrial habitat.

3. Lemmas with 3 prominent nerves.

4. Lemmas pubescent on the nerves and callus, the midnerve extended as a short awn.

5. Palea densely long-ciliate on upper half of keel; panicle with few spreading, few-flowered branches; sandy beach habitat. Expected (Triplasis).

5. Palea not long-ciliate on upper half of keel; panicle large and open; roadside-old field habitat...................... Triodia

4. Lemmas not pubescent on the nerves and callus, awnless .... Eragrostis

3. Lemmas with 5 to many nerves, these sometimes faint.

6. Lemmas keeled.

7. Spikelets with I-4 empty lemmas below the fertile florets .... Uniola

7. Spikelets with no empty lemmas below the fertile florets.

8. Spikelets flat, nearly sessile, in thick I-sided clusters at the ends of stiff branches......................... Dactylis

8. Spikelets only slightly flattened, not in I-sided clusters.

9. Spikelets more than I $\mathrm{cm}$ long; lemmas awned....... Bromus

9. Spikelets not more than $6 \mathrm{~mm}$ long; lemmas awnless .... Poa

6 . Lemmas rounded on back.

Io. Nerves of lemma prominent, parallel. (Also, glumes not papery; all florets alike; spikelets usually green) $\ldots \ldots \ldots \ldots \ldots$. Glyceria

Io. Nerves of lemma less prominent, converging toward the summit.

II. Lemmas minutely bifid at apex, awned from between the teeth

II. Lemmas acute, awned from the tip or awnless.

I2. Lemmas awned (except in F. elatior and F. obtusa); without cobwebby pubescence at base .............. Festuca

I2. Lemmas awnless; with cobwebby pubescence at base (Except in P. annua, P. autumnalis, and P. compressa) .... Poa

\section{Distichlis}

Distichlis spicata (L.) Greene

Saltgrass

(Alkali-grass)

Java Farm and Ivy Neck. Moderately abundant in the more inland parts of salt marshes. Associate species: Spartina patens, Panicum virgatum, Iva frutescens. Higman 52. 
Java Farm. One station in Hog Island salt marsh. Hectare 3583. Associate species: Spartina cynosuroides, Panicum virgatum. One other station reported in Corn Island salt marsh. Higman 44I.

\section{Tridens}

(Triodia)

Tridens flavus (L.) Hitchc. var. flavus

Purpletop Tridens

(Triodia flava (L.) Smyth (Tridens flavus (L.) Hitchc.)

(Fall Redtop)

Java Farm. One station: old field near Fox Point Road on north side, near stand of Pinus virginiana. Hectare 3533. Higman 363.

T. flavus (L.) Hitchc. var. flavus

Purpletop Tridens

(T. flava, forma cuprea (Jacq.) Fosberg)

(Fall Redtop)

Java Farm. Infrequent in old fields. Also one station in cracked pavement at main buildings. Hectare 2580. Associate species: Euphorbia maculata, Juncus tenuis, Eupatorium serotinum. Higman I3, 226, 34I.

Eragrostis spectabilis (Pursh) Steud. $\quad$ Progrostis $\quad$ Purple Lovegrass (Love-grass, Tumble-grass)

Java Farm. Infrequent to moderately abundant in Fox Point Road, Associate species: Plantago aristata, Dianthus armeria, Digitaria ischaemum, Juncus tenuis. Higman 26, 365.

\section{Chasmanthium}

(Uniola)

Chasmanthium laxum (L.) Yates

(Uniola laxa (L.) BSP.)

(new comb. Chasmanthium laxum (L.) Yates)
Slender Woodoats

(Spikegrass, Spanglegrass)

Java Farm. Infrequent in Hog Island salt marsh. Associate species: Distichlis spicata, Panicum virgatum. Higman 342, 452.

Dactylis glomerata L.

$$
\text { Dactylis }
$$

Orchardgrass

(Orchard-grass)

Java Farm. Infrequent: stations beside old and new entrance roads. Associate species: Galium aparine, Carex blanda, Carex muhlenbergii var. enervis, Fragaria virginiana, Poa pratensis. Higman 640, II07. 


\section{Bromus}

I. Spikelets strongly flattened, lemmas keeled; awn short or wanting. Expected (B. catharticus).

I. Spikelets not strongly flattened, terete before flowering; awn usually present.

2. Plants perennial; culms usually I $m$ tall or more.

3. Creeping rhizomes present; panicle erect, contracted at maturity; lemmas glabrous; awn minute or wanting . ............ inermis

3. Creeping rhizomes wanting; panicle open, spreading or drooping; lemmas evenly pubescent; awn present. Expected (B. latiglumis).

2. Plants annual; culms usually shorter than $70 \mathrm{~cm}$

4. Lemmas narrow, acuminate, with a sharp callus at the base; awns usually more than I. $5 \mathrm{~cm}$ long. Expected (B. tectorum).

4. Lemmas broad, rounded above, without a sharp callus; awns usually less than I cm long or wanting.

5. Panicle contracted, its branches erect or ascending.

6. Lemmas pubescent. Expected (B. mollis).

6. Lemmas scabrous or glabrous ............... B. racemosus

5. Panicle open, its branches spreading or drooping.

7. Lemma inflated, awnless. Expected (B. brizeaformis).

7. Lemma not inflated, awned.

8. Branches of panicle rather stiffly spreading, not drooping

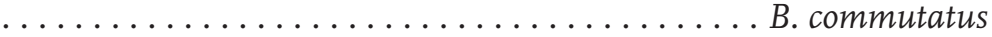

8. Branches of panicle lax, drooping.

9. Lemmas obtuse; awn flexuous; palea distinctly shorter than lemma ....................... japonicus

9. Lemmas acute, bifid; awn straight or nearly so; palea about as long as lemma. Expected (B. arvensis).

Bromus inermis Leyss.

Smooth Brome

(Awnless Bromegrass)

Ivy Neck. One station: beside trail to abandoned grassy field on Scaffold Peninsula. Hectare 4740. Associate species: Rumex crispus, Anagallis arvensis, Galinsoga ciliate, Mollugo verticillata, Oenothera biennis. Higman 854.

B. racemosus L.

Bald Brome

(Bromegrass)

Java Farm. One station: broken pavement in Fox Point Road. Associate species: Plantago aristata, Festuca myuros, Cyperus filicinus. Higman iıı2. 
B. racemosus L.

(B. commutatus Schrad.)

(B. racemosus L.)
Bald Brome

(Hairy Chess)

Java Farm. Infrequent along new entrance road and Fox Point Road. Also one station on south-facing slope east of Muddy Creek and adjacent north border. Hectare 2458. Associate species: Allium vineale, Juncus canadensis, Carex tribuloides, Plantago aristata. Higman 788, 891, II36.

B. arvensis $\mathrm{L}$.

Field Brome

(B. japonicus Thunb.)

(Japanese Chess)

Java Farm. One station: old field on south side of Fox Creek marsh. Hectare 3524. Partial canopy of Pinus virginiana and Liquidambar styraciflua. Associate species: Erigeron annuus, Solidago graminifolia, S. altissima. Higman 890.

Poa

I. Plants not more than $25 \mathrm{~cm}$ tall; annuals.

2. Lemmas with webby pubescence at base, distinctly 3-nerved, the intermediate nerves faint, thickly pubescent on the lateral nerves and keel; plants pale

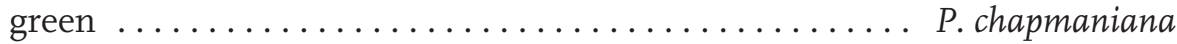

2. Lemmas glabrous at base, distinctly 5-nerved, somewhat pubescent on the lower half of all nerves, especially the keel; plants bright green .... P. annua

I. Plants more than $25 \mathrm{~cm}$ tall; perennials.

3. Creeping rhizomes present.

4. Culms very flattened, 2-edged; lemmas glabrous or nearly so at base ....

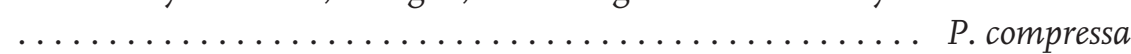

4. Culms rounded or slightly flattened; lemmas with webby pubescence at base.

5. Blades shorter than the culm; lower branches of panicle in a whorl of 5

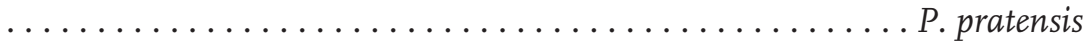

5. Blades about equal to the culm in length; lower branches of panicle usually in 2's. Expected (P. cuspidata).

3. Creeping rhizomes wanting.

6. Lemmas glabrous at base, tips of lemma blunt and scarious . . . . . . . .

P. autumnalis

6. Lemmas webbed at base.

7. Lemmas slightly pubescent on the keel or glabrous on all nerves. Expected ( $P$. trivialis).

7. Lemmas pubescent on the keel and marginal nerves.

8. Lower panicle branches spreading or reflexed at maturity; panicle erect, much longer than wide; intermediate nerves of lemma prominent ........................ sylvestris

8. Lower panicle branches not reflexed at maturity; panicle long, open; intermediate nerves of lemma faint. Expected (P. palustris). 
Java Farm. One station: broken pavement at main building area. Hectare 2580. Associate species: Poa annua, Juncus tenuis, Triodia flava.

Ivy Neck. One station at border of cornfield on Scaffold Peninsula. Higman 587, 822.

P. annua L.

Annual Bluegrass

Java Farm. One station: broken pavement at main building area. Hectare 2580 . Associate species: Poa chapmaniana above. Higman iıız.

P. compressa L.

Canada Bluegrass

(Wiregrass, Canada bluegrass)

Ivy Neck. Moderately abundant in grazed woods at head of Cheston Creek estuary. Canopy of Robinia pseudoacacia, Platanus occidentalis, Diospyros virginiana. Also one station on east bank of estuary. Higman 731, 734, 876.

P. pratensis L.

Kentucky Bluegrass

(Junegrass)

Java Farm. Moderately abundant; dominant species in the few remaining grass areas in old fields. Infrequent along roadsides at both Java Farm and Ivy Neck. Forms dense turf, invaded by Rhus radicans and Cirsium discolor. Higman 335, 547, 62I.

P. autumnalis Muhl. ex Elliott

Autumn Bluegrass (P. autumnalis Muhl. ex Ell.)

(Meadowgrass)

Java Farm. One station: Mature forest near old entrance road. Hectare 2474. Canopy of Fagus grandifolia, Quercus alba, Nyssa sylvatica, Liriodendron tulipifera, Liquidambar styraciflua. Higman 678.

P. sylvestris A. Gray

(P. sylvestris Gray)

Woodland Bluegrass

(Meadowgrass)

Java Farm. Infrequent; stations at old entrance gate and at bank of Muddy Creek upstream from entrance road. Canopy of mature Fagus grandifolia, Liriodendron tulipifera. Associate species (none at both stations): Dactylis glomerata, Galium aparine, Glyceria striata. Higman 64I, 79I.

\section{Glyceria}

I. Spikelets linear, $\mathrm{I}-4 \mathrm{~cm}$ long $\ldots \ldots \ldots \ldots \ldots \ldots$. septentrionalis

I. Spikelets ovate, $2-7 \mathrm{~mm}$ long. (Also, panicle open; lemmas prominently nerved; first glume less than I mm long; florets crowded toward ends of branches, often

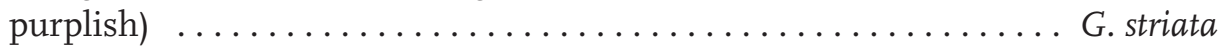


Ivy Neck. One station: moist, grazed woods at head of Cheston Creek estuary. Hectare 4733. Canopy of mature Platanus occidentalis, Diospyros virginiana, Liquidambar styraciflua, Robinia pseudoacacia. Associate species: Glyceria striata, Poa compressa, Carex crinita, Carex tribuloides. Higman 735.

G. striata (Lam.) Hitchc.

Fowl Mannagrass

(Fowl Meadowgrass)

Java Farm and Ivy Neck. Infrequent to moderately abundant in stream valleys, moist woodland, etc. Principal stations are at head of Cheston Creek estuary (described above) and at shore of Muddy Creek, upstream from culvert of old entrance road (Java Farm). Associate species besides those listed above: Panicum clandestinum, Lindera benzoin, Carex blanda, Duchesnea indica, Danthonia spicata. Higman I80, 730, 732, 745, 756, 790 .

\section{Schedonorus, Festuca, Vulpia}

I. Blades usually 4-8 mm wide, flat. (Also, plants tall perennials, not densely tufted; lemmas awnless except in F. elatior forma aristata).

2. Spikelets 8-I5 mm long, 6- to II-flowered; panicle contracted; lemmas acute

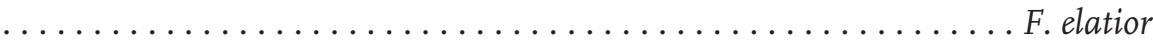

2. Spikelets 4-6 mm long, 2- to 5-f lowered; panicle diffuse; lemmas obtuse.

3. Spikelets lanceolate, scattered at tips of branchlets; glumes and lemmas appressed. Expected (F. obtusa).

3. Spikelets ovate, close together; glumes and lemmas loosely ascending... $\ldots \ldots \ldots \ldots \ldots \ldots \ldots \ldots \ldots \ldots \ldots \ldots \ldots \ldots \ldots \ldots \ldots \ldots \ldots \ldots$

I. Blades usually less than I.5 mm wide, involute or, if flat, less than $3.5 \mathrm{~mm}$ wide. 4. Plants annual; stamen I. (Also, lemmas glabrous).

5. First glume much shorter than the second; awns about I $\mathrm{cm}$ long. . . . . . .

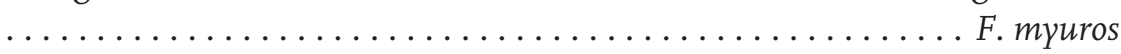

5. First glume $2 / 3$ to $3 / 4$ as long as the second; awns 3 to $5 \mathrm{~mm}$ long ......

4. Plants perennial; stamens 3. (Also, culms densely tufted).

6. Culms somewhat decumbent at base; base reddish, the basal sheaths becoming fibrous shreds ................... F. rubra

6. Culms erect; base not reddish or shreding. Expected (F. ovina).

Schedonorus pratensis (Huds.) P. Beauv.

Meadow Fescue (Festuca elatior L.)

Meadow-Fescue

Ivy Neck. One station: abandoned grassy field on Scaffold Peninsula. Hectare 4772. Associate species: Phleum pratense, Trifolium arvense, Hypericum perforatum. Higman 809 . 
Java Farm. One station: beside new entrance road. Hectare 2582. Associate species: Panicum clandestinum, Vicia sativa, Lepidium campestre, Melilotus alba. Higman 833.

Vulpia myuros (L.) C.C. Gmel.

Annual Fescue

(F. myuros L.)

Java Farm. One station: broken pavement on Fox Point Road. Associate species: Plantago aristata, Cyperus filicinus, Dianthus armeria. Higman 807.

V. octoflora (Walter) Rydb. var. octoflora

(F. octoflora Walt.)
Sixweeks Fescue

(Six-weeks Fescue)

Ivy Neck. One station: west shore of Cheston Creek estuary. Canopy of Quercus velutina, Acer rubrum, Juglans nigra, Liquidambar styraciflua. Associate species: Glyceria striata. Higman $74^{2}$.

F. rubra L.

Red Fescue

Java Farm. Infrequent; road to springhouse. Hectare 34I7. Associate species: Taraxacum officinale, Poa pratensis, Rhus radicans. Higman iıog, IIIo.

\section{Andropogoneae}

I. Spikelets all alike, perfect. (Also, spikelets surrounded by copious soft hairs; lemmas awned). Expected (Miscanthus, Erianthus).

I. Spikelets dissimilar, one of each group sessile and perfect, the other I or 2 pedicellate and either staminate, sterile, or reduced to a pedicel.

2. Blades ovate; plant a weak, trailing annual. Expected (Arthraxon).

2. Blades linear, elongate; plant erect, stiff, perennial.

3. Racemes terminal and axillary; rachis with several to many joints, promptly

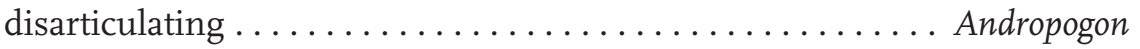

3. Racemes terminal only; rachis with 2 or 3 tardily separating joints.

4. Fertile spikelet awnless (awn very early-deciduous); pediceled spikelets

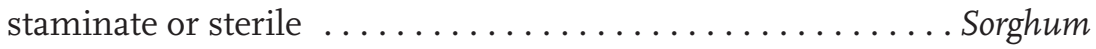

4. Fertile spikelet with a long, persistent, basally twisted awn; pediceled spikelets usually rediced to hairy pedicels .......... Sorghastrum

\section{Schizachyrium, Andropogon}

I. Racemes solitary on each peduncle; internodes and pedicels club-shaped.....

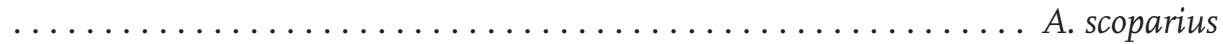

I. Racemes 2 to several on each peduncle, digitate; internodes and pedicels slender. (Also, pedicellate spikelet reduced to a pedicel or to empty glumes; racemes silky villous, not conspicuously aggregate at the summit of the culm). 
2. Peduncles mostly 5 to I $5 \mathrm{~cm}$ long. Expected (A. elliottii, A. ternarius).

2. Peduncles not more than I $\mathrm{cm}$ long.

3. Upper sheaths conspicuously inflated. Expected (A. elliottii).

3. Upper sheaths not inflated .................. virginicus

\section{Schizachyrium scoparium (Michx.) Nash var. scoparium} (Andropogon scoparius Michx.)

Little Bluestem (Bluestem, Bunchgrass)

Java Farm. Infrequent in Hog Island salt marsh. Associate species: Panicum virgatum, Myrica cerifera, Diospyros virginiana, Distichlis spicata. Higman 287, 292, 302.

Andropogon virginicus L.

Broomsedge Bluestem

(Broomsedge)

Java Farm. Infrequent in Phalaris arundinacea meadow and on adjacent southand west-facing slopes. Associate species: Asplenium platyneuron, Hypericum mutilum, Paspalum laeve, Sorghastrum nutans. Higman I50.

Sorghum

Sorghum halepense (L.) Pers.

Johnsongrass

(Johnson-grass)

Java Farm. Infrequent in old fields, especially near the pier. Associate species: Gnaphalium obtusifolium, Triosetum perfoliatum, Solidago altissima. Higman I44, 243.

Sorghastrum nutans (L.) Nash

Sorghastrum

Indiangrass

(Indian Grass)

Java Farm. Infrequent in old fields; often solitary among dense Lonicera japonica and Rhus radicans. Higman 205, 207, 467.

\section{Paniceae}

I. Spikelets subtended by an involucre of I to many distinct or united bristles.

2. Bristles united into a spiny bur permanently enclosing the spikelet ......

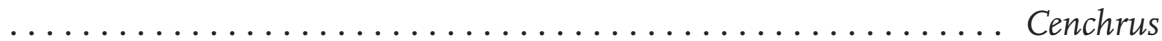

2. Bristles separate, persistent, the spikelets deciduous .......... Setaria

I. Spikelets not subtended by an involucre.

3. Glumes or sterile lemma awned ................. Echinochloa

3. Glumes and sterile lemma awnless.

4. Fruit tough but flexible; margins of lemma not inrolled.

5. Spikelets in slender, rather digitate terminal racemes ...... Digitaria

5. Spikelets in diffuse panicles. Expected (Leptoloma). 
4. Fruit hard and rigid; margins of lemma inrolled. (Also, spikelets placed with the back of the lemma toward the rachis, or spikelets pedicellate in panicles).

6. First glume wanting; spikelets plano-convex, in spikelike racemes ....

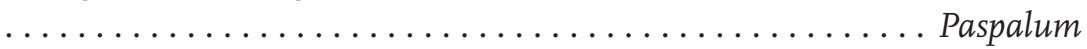

6. First glume present; spikelets not plano-convex, in panicles.

7. Spikelets on short pedicels; second glume inflated like a sac near the base. Expected (Sacciolepis).

7. Spikelets on long pedicels; second glume not inflated ..... Panicum

Cenchrus

Cenchrus tribuloides L.

Sanddune Sandbur

(Sandbur)

Ivy Neck. Infrequent on sandy beach on Rhode River shore of Cheston Peninsula. Associate species: Strophostyles helvola, Spartina alterniflora, Cakile edentula. Higman IOI3.

\section{Setaria}

I. Bristles of involucre at least 5. (Also, panicle dense, spikelike).

2. Plants with short, knotty rhizomes; spikelets about $2.5 \mathrm{~mm}$ long; palea reduced; plant perennial. Expected (S. geniculata).

2. Plants without rhizomes; spikelets about $3 \mathrm{~mm}$ long; palea developed; plant

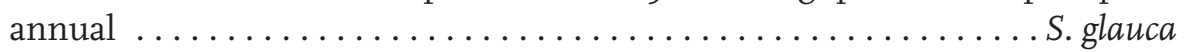

I. Bristles of involucre I to 3 , or appearing more by abortion of spikelets.

3. Bristles retrorsely scabrous. Expected (S. verticillata).

3. Bristles antrorsely scabrous.

4. Fertile lemma coarsely transversely rugose; spikelets about $3 \mathrm{~mm}$ long

4. Fertile lemma smooth or slightly rugose; spikelets not more than $2.5 \mathrm{~mm}$ long. Expected (S. viridis).

Setaria pumila (Poir.) Roem. \& Schult. subsp. pumila

Yellow Foxtail (Setaria glauca (L.) Beauv.)

(S. lutescens (Weigel) F. T. Hubbard)

(Foxtail Grass, Yellow bristlegrass)

Java Farm. Infrequent in old fields.

Ivy Neck. Moderately abundant near margins of cultivated fields. Associate species: Strophostyles umbellata, Echinochloa walteri, Polygonum persicaria, Eupatorium serotinum, Datura stramonium. Higman I40, 336, I045, I052, II27. 
Java Farm. One station: rubbish pile at main building area. Hectare 2580. Associate species: Eleusine indica, Datura stramonium. Also one station at north border, beside neighboring cornfield.

Associate species: Clematis virginiana, Amaranthus hybridus.

Ivy Neck. One station: pile of dry soil near artificial pond at head of Cheston Creek drainage. Hectare 4722. Associate species: Echinochloa pungens. Higman 5, 878, I035.

\section{Echinochloa}

I. Second glume awnless (often acuminate in E. pungens); spikelets ovoid; fruit ovoid to oval; sheaths of leaves glabrous.

2. Fertile (coriaceous) lemma obtuse to subacute, the tip soft and wilting; spikelets with appressed slender trichomes, pustular-based trichomes if present few and marginal. Expected (E. crusgalli).

2. Fertile lemma acuminate, the tip firm; spikelets with conspicuous bristles, the trichomes usually pustular-based .............. pungens

I. Second glume awned (awn shorter than that of sterile lemma); spikelets ellipsoid; fruit slenderly lanceolate to lance-ellipsoid, acute; sheaths of leaves usually hirsute, the hairs pustular-based ................ walteri

Echinochloa muricata (P. Beauv.) Fernald var. muricata (Echinochloa pungens (Poir.) Rydb.)

Rough Barnyardgrass

(Barnyard Grass) (E. crusgalli (L.) Beauv.)

Java Farm. One station: marshy west end of Phalaris arundinacea meadow, north of main building area. Hectare 2467. Associate species: Erechtites hieracifolia, Juncus effusus, Polygonum pensylvanicum, Polygonum sagittatum.

Ivy Neck. Infrequent at borders of cultivated fields: one station near artificial pond at source of Cheston Creek drainage; one in weedy field near forested valley of Scaffold Creek drainage.

Associate species: Eleusine indica, Ludwigia palustris, Polygonum persicaria, Setaria glauca, Strophostyles umbellata. Higman I74, 255, 866, 996, II38.

E. walteri (Pursh) A. Heller

Coast Cockspur Grass

(E. walteri (Pursh) Nash)

Java Farm. One station: upstream limit of salt marsh on Muddy Creek, beyond margin of forest. Hectare 3430. Associate species: Echinochloa pungens, Baccharis halimifolia, Cyperus strigosus, Asclepias incarnata, Eclipta alba, Scirpus validus var. creber. Higman 54I. 
Java Farm. Infrequent along Fox Point Road and in yard of burned house on road to springhouse. Associate species: Juncus tenuis, Rhus typhina, Paspalum setaceum. Higman 27, 387 .

\section{Paspalum}

I. Racemes both terminal and axillary, the latter sometimes hidden in the sheaths; spikelets not more than $2 \mathrm{~mm}$ long.

2. Blades usually not over $8 \mathrm{~mm}$ wide; spikelets about I. $5 \mathrm{~mm}$ long ......... ....................................... setaceum

2. Blades up to $20 \mathrm{~mm}$ wide; spikelets at least $2 \mathrm{~mm}$ long. (Also, culms erect; leaves sparsely to densely pilose). Expected (P. pubescens).

I. Racemes terminal on the primary culm or on leafy branches, not axillary; spikelets $2.5-4 \mathrm{~mm}$ long.

3. Spikelets conspicuously ciliate on the margins; leaves glabrous. (Also, culms geniculate at base). Expected (P. dilatatum).

3. Spikelets not ciliate; leaves pilose.

4. Plants robust, usually $\mathrm{I}-2 \mathrm{~m}$ tall; spikelets $4 \mathrm{~mm}$ long ...... P. floridanum.

4. Plants not robust, less than I $\mathrm{m}$ tall; spikelets usually under $3.5 \mathrm{~mm}$ long.

5. Spikelets orbicular, $3-3.2 \mathrm{~mm}$ long. (Also, blades as long as or longer than the culm). Expected (P. circulare).

5. Spikelets longer than broad, mostly under $3 \mathrm{~mm}$ long.

6 . Sheaths and blades very pilose; culms ascending to erect. Expected (P. longipilum).

6. Sheaths and blades glabrous or sparsely pilose; culms more spreading ...................................... P. leave

Paspalum setaceum Michx.

Thin Paspalum

Java Farm. One station: yard of burned house on road to springhouse. Hectare 34I7. Partial canopy of Rhus typhina. Associate species: Digitaria ischaemum, Lonicera japonica, Trifolium repens, Lepidium virginicum, Clematis virginiana. Higman 386.

P. floridanum Michx.

Florida Paspalum

(P. floridanum Michx., var. glabratum Engelm. ex Vasey)

Java Farm. Infrequent: one station on Fox Point Road; associate species: Juncus tenuis, Eragrostis spectabilis, Plantago aristata. Also one station in Hog Island salt marsh; associate species:

Distichlis spicata, Andropogon scoparius, Panicum virgatum, Juncus dichotomus. Higman I8, 299. 
Java Farm. One station: south-facing slope north of Phalaris arundinacea meadow, north of main building area. Hectare 2458. Spreading Rhus radicans, Campsis radicans, and Rubus spp.: few grassy spots. Associate species: Lespedeza repens, Hieracium scabrum. Higman ${ }_{6} 5$.

\section{Panicum, Dichanthelium}

I. Basal leaves similar to culm leaves, not forming winter rosettes.

2. Plants annual.

3. Spikelets warty, tuberculate. Expected (P. verrucosum).

3. Spikelets smooth.

4. First glume about I/4 as long as the spikelet, blunt, rounded, or truncate; sheaths glabrous; nodes glabrous........... P. dichotomiflorum

4. First glume about $\mathrm{I} / 2$ as long as the spikelet, acute or acuminate; sheaths hirsute or villous; nodes bearded. (Also, panicle erect; spikelets not more than $4 \mathrm{~mm}$ long).

5. Panicle more than $\mathrm{I} / 2$ the entire length of plant. (Also, panicle as broad as long) spikelets $2-2.5 \mathrm{~mm}$ long .......... P. capillare

5. Panicle not more than $\mathrm{I} / 3$ the entire length of plant. Expected (P. philadelphicum).

2. Plants perennial.

6. Plants without creeping, scaly rhizomes. Expected (P. agrostoides, P. stipitatum).

6. Plants with creeping, scaly rhizomes.

7. Pedicels of spikelets usually over $3 \mathrm{~mm}$ long.

8. Panicle long and narrow; spikelets $5-6.5 \mathrm{~mm}$ long; sandy seacoast habitat. Expected (P. amarum).

8. Panicle moderately diffuse; spikelets $4.5 \mathrm{~mm}$ long; salt marsh and tidal stream habitat. (Also, culms rigid, terete; sheaths not compressed) .................... P. virgatum

7. Pedicels of spikelets I-3 mm long. (Also, panicle open; spikelets 3.3$3.7 \mathrm{~mm}$ long; culms firm to soft; culms and sheaths compressed; terrestrial habitat) $\ldots \ldots \ldots \ldots \ldots \ldots \ldots \ldots \ldots \ldots \ldots \ldots \ldots \ldots \ldots$ anceps

I. Basal leaves unlike the cauline ones, forming winter rosettes (except in P. depauperatum, the leaves then crowded near the base).

9. Spikelets glabrous or nearly so.

Io. Spikelets about $3.5 \mathrm{~mm}$ long. (Also, sheaths pilose; blades scabrous above, pilose beneath). Expected ( $P$. depauperatum).

Io. Spikelets less than $3 \mathrm{~mm}$ long.

II. Spikelets I. $5 \mathrm{~mm}$ long or less. (Also, ligule not over I.5 $\mathrm{mm}$ long; nodes bearded) ................... microcarpon

II. Spikelets 2-3 $\mathrm{mm}$ long. Expected (5 species, especially P. dichotomum). (Also, blades $2 \mathrm{~cm}$ wide or more, strongly nerved, scabrous, round at base; spikelets $3 \mathrm{~mm}$ long). 
9. Spikelets pubescent, some sparingly so.

I2. Spikelets $3 \mathrm{~mm}$ long or more.

I3. Blades of leaves at midculm I5 times as long as wide. (Also, sheaths hispid or glabrous; spikelets pointed). Expected (P. depauperatum).

I3. Blades of leaves at midculm seldom over io times as long as wide. (Also, spikelets 3.5-4 $\mathrm{mm}$ long or more).

I4. Blades velvety-pubescent beneath. Expected (P. ravenelii, P. boscii, P. molle).

I4. Blades not velvety-pubescent beneath.

15. Sheaths glabrous or minutely puberulent.

I6. Nodes bearded; spikelets $4 \mathrm{~mm}$ long or more. Expected (P. boscii).

I6. Nodes not bearded; spikelets about $2.8 \mathrm{~mm}$ long...... .......................... P. commutatum

I5. Sheaths bristly. (Also, blades $2 \mathrm{~cm}$ wide or more) ........ ............................ P. clandestinum

I2. Spikelets less than $3 \mathrm{~mm}$ long. (Also, blades usually over $5 \mathrm{~mm}$ wide).

I7. Spikelets attenuate at base; blades tapering from base to apex. Expected ( $P$. angustifolium).

I7. Spikelets not attenuate at base; blades otherwise. (Also, sheaths not retrorsely pilose).

I8. Ligule (I.5) 2-5 $\mathrm{mm}$ long.

I9. Sheaths, or all but the lowest, glabrous. (Also, ligule $4-5 \mathrm{~mm}$ long; spikelets I. $5 \mathrm{~mm}$ long: leaf blades and axis of panicle glabrous or nearly so). Expected (P. lindheimeri).

I9. Sheaths pubescent. (Also, culms and spikelets usually so). 20. Spikelets not more than $2 \mathrm{~mm}$ long.

2I. Ligule I-I.5 $\mathrm{mm}$ long. Expected (P. tsugetorum).

2I. Ligule $2-5 \mathrm{~mm}$ long.

22. Axis of panicle glabrous or puberulent. (Also, spikelets I.3-I.6 mm long). Expected (P. meridionale).

22. Axis of panicle pilose.

23. Spikelets I-I.3 $\mathrm{mm}$ long. Expected (P. leucothrix).

23. Spikelets I.6-I.9 $\mathrm{mm}$ long.

24. Culms, sheaths, and blades velvety ... ................ P. lanuginosum

24. Culms, sheaths, and blades spreadingpilose. Expected (P. huachucae).

20. Spikelets about $2.5 \mathrm{~mm}$ long. (Also, culms, sheaths, and blades densely villous). Expected (P. villosissimum). See Appendix I. 
I8.

Ligule absent.

25. Nodes bearded.

26. Spikelets I.3-I.5 mm long .......... P. microcarpon

26. Spikelets $2 \mathrm{~mm}$ long or more. Expected ( $P$. mattamuskeetense).

25. Nodes not bearded.

27. Plant velvet-pubescent throughout. Expected (P. scoparium).

27. Plant not velvety throughout.

28. Sheaths pilose or hispid.

29. Blades $2 \mathrm{~cm}$ wide or more ...... P. clandestinum

29. Blades less than $\mathrm{I} \mathrm{cm}$ wide. Expected (P. addisonii).

28. Sheaths glabrous or puberulent.

29. Spikelets spherical. (Also, blades cordate, whitemargined). Expected (P. polyanthes).

29. Spikelets ovate.

30. Spikelets $2.3-3 \mathrm{~mm}$ long.

30. Spikelets less than $2 \mathrm{~mm}$ long. Expected (P. columbianum).

3I. Culms glabrous or nearly so; blades I. $5 \mathrm{~cm}$ wide or more....P. commutatum

3I. Culms puberulent; blades not over I cm wide. Expected (P. ashei).

Panicum dichotomiflorum Michx.

Fall Panicgrass

Java Farm. One station: border of salt marsh on tidal portion of Muddy Creek. Associate species: Spartina alterniflora, Rumex verticillatus. Higman 290.

P. capillare L.

Witchgrass

(Old-witch Grass)

Java Farm. One station: small old field north of main building area. Hectare 2580. Lonicera japonica and Vitis vulpina dominant. Higman 539.

P. virgatum L. var. spissum Linder

Switchgrass

Java Farm and Ivy Neck. Moderately abundant in salt marshes, especially the more inland parts. Associate species: Distichlis spicata, Pluchea camphorata, Spartina patens. Higman 89, 344, 440, 973, 1027, 1050, 1051.

P. anceps Michx.

Beaked Panicgrass

Java Farm. Infrequent in woods between Muddy Creek and Phalaris arundinacea meadow, north of main building area. Canopy of Fagus grandifolia, Platanus occidentalis, and Quercus alba. Associate species: Lindera benzoin, Glyceria striata, Onoclea sensibilis, Duchesnea indica. Higman I8I, 474, IIII. 
Dichanthelium sphaerocarpon (Elliott) Gould var. isophyllum Roundseed Panicgrass (Scribn.) Gould \& C.A. Clark

(P. microcarpon Muhl.)

Java Farm. Infrequent in Hog Island salt marsh, near landward margin. Associate species: Panicum virgatum, Cyperus filicinus, Juncus dichotomus.

Ivy Neck. Infrequent on west shore of Cheston Creek. Associate species: Danthonia spicata, Hieracium venosum, Panicum commutatum, Rosa Carolina. Higman 303, 446,847 .

D. commutatum (Schult.) Gould

Variable Panicgrass

(P. commutatum Schult.)

Ivy Neck. One station: west shore of Cheston Creek. Canopy of Quercus prinus, Quercus alba, Associate species: Danthonia spicata, Hieracium venosum, Panicum microcarpon. Higman 848 .

D. clandestinum (L.) Gould

Deertongue

(P. clandestinum L.)

Java Farm. Infrequent along Muddy Creek valley and Fox Creek drainage. Associate species: Geum canadense, Carex blanda, Danthonia spicata, Muhlenbergia schreberi, Perilla frutescens. Higman 87, 308, 322, 785, 832.

D. acuminatum (Sw.) Gould \& C.A. Clark var. fasciculatum Western Panicgrass (Torr.) Freckmann

(Panicum lanuginosum Ell., var. fasciculatum (Torr.) Fern.)

Ivy Neck. One station: abandoned unpaved road through forest on Scaffold Peninsula. Canopy of Quercus alba, Quercus velutina, Fagus grandifolia, Liriodendron tulipifera. Associate species: Houstonia purpurea, Juncus tenuis, Vernonia glauca. Higman 84I.

\section{CYPERACEAE}

I. Flowers unisexual, the staminate and pistillate in the same or in different spikes (the latter resembling spikelets).

2. Achene naked, bony, supported on a disc. Expected (Scleria).

2. Achene enclosed in a sac (perigynium) borne in the axil of a bract or scale. (Also, culms mostly triangular; leaves with sheath, ligule, and midrib; spikes in axils of leafy or scalelike bracts, often aggregated into heads) . . . . . Carex

I. Flowers perfect, spikes essentially uniform.

3. Spikelets I-2-flowered, with 2-many empty basal scales. Expected (Rhynchospora). 
3. Spikelets many-flowered (if only I-flowered, the spikelets in densely glomerulate heads with 2-ranked scales), with I of the lower scales empty (more in Eriophorum).

4. Scales of spikelet strictly 2-ranked, folded lengthwise and keeled.

5. Inflorescences terminal simple or compound umbels or glomerules; flowers without perianth bristles; achene without a tubercle or enlarged style-base .............................. Cyperus

5. Inflorescences axillary, from leaf sheaths; flowers with a perianth of bristles; achene capped by a long tubercle. Expected (Dulichium).

4. Scales of spikelet spirally arranged (sometimes 2-ranked in Eleocharis, with single terminal spikelets).

6 . Achene crowned by a persistent bulbous tubercle.

7. Culms naked, the basal colored sheaths usually bladeless; spikelet terminal; perianth of bristles often present........... Eleocharis

7. Culms leafy or leafy-based; spikelets 2-many, in terminal or axillary inflorescences with leafy involucres; perianth wanting. Expected (Bulbostylis, Psilocarya).

6. Achene without a tubercle (Scirpus may have a small point on the achene, left by the fallen style).

8. Flowers with one or more inner subtending scales or with dilated sepals. Expected (Hemicarpha).

8. Flowers without inner subtending scales or dilated sepals (may have bristle perianth).

9. Style dilated at base, deciduous below the enlargement; perianth wanting. Expected (Fimbristylis).

9. Style terete, slender; perianth usually present.

Io. Perianth of I to 6 broad bristles. (Also, spikelets with an involucre of I erect or several spreading leaves) ........ Scirpus

Io. Perianth of many long silky bristles. Expected (Eriophorum).

\section{Carex}

(from Hermann, I94I)

Key to Sections

I. Spike one.

2. Spikes with pistillate flowers above, staminate ones below, many-flowered; perigynia strongly inflated, sessile .............. Squarrosae (p. 8I)

2. Spikes with staminate flowers above, pistillate ones below; perigynia not inflated. Expected (Phyllostachyae, Polytrichoideae). Species in expected sections are listed on p. 87.

I. Spikes more than one.

3. Stigmas two; achenes lenticular.

4. Lateral spikes sessile, short; terminal spike usually with both pistillate and staminate flowers. 
5. Culms arising singly or few-together from long-creeping rootstocks. Expected (Divisae, Arenariae).

5. Culms tufted, the rootstocks occasionally prolonged with short internodes but not long-creeping.

6. Spikes with staminate flowers above, pistillate ones below.

7. Perigynia tapering into the beak or, if abruptly contracted, the culms flaccid and flattening in drying. Expected (Vulpinae).

7. Perigynia abruptly contracted into the beak; culms not flaccid nor flattening in drying.

8. Spikes usually io or fewer, greenish........ Bracteosae (p. 82)

8. Spikes numerous, yellowish or brownish at maturity; leafsheaths often red-dotted ventrally.

9. Perigynia plano-convex, thin, yellowish; bracts mostly much exceeding the spikes; leaf-sheaths usually transversely rugose ventrally ................. Multiflorae (p. 84)

9. Perigynia thick-plano-convex or unequally biconvex, brown; bracts mostly shorter than the spikes; leaf-sheaths not transversely rugose. Expected (Paniculatae).

6. Spikes with pistillate flowers above, staminate ones below.

Io. Perigynia with winged margins ............. Ovales (p. 84)

Io. Perigynia without winged margins, at most thin-edged. Expected (Stellulatae, Deweyanae, Heleonastes).

4. Lateral spikes peduncled or, if sessile, elongate; terminal spike usually staminate.

II. Achenes constricted in the middle; scales 3-nerved, long-awned..... ............................... Cryptocarpae (p. 85)

II. Achenes not constricted in the middle; scales I-nerved, not longawned. Expected (Acutae).

3. Stigmas three; achenes trigonous.

I2. Perigynia or foliage pubescent.

I3. Style continuous with the achene, persistent, indurated. Expected (Paludosae).

I3. Style articulated with the achene, at length deciduous.

I4. Achenes closely enveloped by the perigynia; bracts sheathless or nearly so.

15. Perigynia obtusely triangular or orbicular-triangular in crosssection; plant (except perigynia) glabrous .............

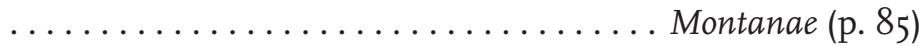

I5. Perigynia sharply triangular in cross-section; plant pubescent. Expected (Triquetrae).

I4. Achenes not closely enveloped by the perigynia or, if so, the bracts strongly sheathing. 
I6. Beak of perigynium strongly bidentate; leaves septate-nodulose. Expected (Hirtae).

I6. Beak of perigynium at most shallowly bidentate; leaves not septatenodulose.

I7. Bracts long-sheathing, or at least the lower ones $\ldots \ldots \ldots \ldots$ Laxiflorae (p. 85)

I7. Bracts sheathless or the lower ones short-sheathing $\ldots \ldots \ldots \ldots$ $\ldots \ldots \ldots \ldots \ldots \ldots \ldots$ Virescentes $(p .87$.

I2. Perigynia and foliage glabrous.

I8. Style not articulated, continuous with the achene, persistent, indurated.

I9. Perigynia subcoriaceous, firm. Expected (Paludosae).

I9. Perigynia membranaceous.

20. Perigynia obconic or broadly obovoid, truncately contracted into long, subulate beaks ........... Squarrosae (p. 8I)

20. Perigynia lanceolate to ovoid or globose-ovoid, not truncately contracted.

2I. Perigynia lanceolate or ovoid-lanceolate, tapering into the beak. Expected (Lupulinae, Collinsiae, Folliculatae).

2I. Perigynia broader, abruptly contracted into the beak, usually strongly ribbed.

22. Perigynia finely and closely ribbed. Expected (Pseudocyperi).

22. Perigynia coarsely ribbed.

23. Perigynia 7-Io $\mathrm{mm}$ long; achenes $2-3 \mathrm{~mm}$ long, I.25-2.25 mm wide ......... Vesicariae (p. 87)

23. Perigynia Io-20 $\mathrm{mm}$ long; achenes $2.5-6 \mathrm{~mm}$ long, 2-4 mm wide. Expected (Lupulinae).

I8. Style articulated with the achene, at length deciduous.

24. Achenes strongly constricted at base, rounded at apex; lower pistillate scales bract-like. Expected (Phyllostachyae).

24. Achenes not strongly constricted at base, short-pointed at apex; lower pistillate scales not bract-like.

25. Lower bracts sheathless or short-sheathing. Expected (Shortianae, Atratae, Gracillimae).

25. Lower bracts long-sheathing.

26. Bracts bladeless or with rudimentary blades

26. Bracts with well-developed blades.

Laxiflorae (p. 85)

27. Foliage, especially the sheaths, pubescent or puberulent. Expected (Gracillimae, Sylvaticae).

27. Foliage glabrous. 
28. Beak of perigynium bidentate. Expected (Extensae).

28. Beak of perigynium at most emarginate.

29. Pistillate spikes elongate, linear to cylindric, on slender peduncles, the lower usually drooping; perigynia not sharply trigonous. Expected (Gracillimae, Sylvaticae).

29. Pistillate spikes short, oblong or linear, erect or, if drooping, either on long capillary peduncles or with the perigynia sharply trigonous. 30. Perigynia with numerous fine impressed nerves. Expected (Oligocarpae, Griseae).

30. Perigynia with few to many strongly raised nerves.

3I. Perigynia rounded at the base, suborbicular in cross-section, loosely enveloping the achenes. Expected (Granulares).

3I. Perigynia tapering at the base, trigonous, closely enveloping the achenes.

32. Rootstocks elongate, often with long horizontal stolons. Expected (Paniceae).

32. Rootstocks not elongate, without long horizontal stolons .......... Laxiflorae (p. 85)

\section{Section Squarrosae}

Keys to the Species

I. Perigynia shorter than the rough-awned scales, I4-20 ribbed; terminal spike usually staminate, narrowly linear, $0.5-2.5 \mathrm{~mm}$ long; ligule not longer than wide

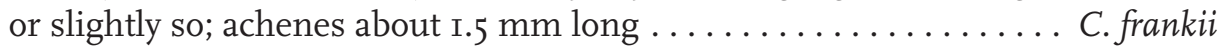

I. Perigynia much longer than the scales, several-ribbed above; terminal spike with pistillate flowers above, staminate ones below; ligule much longer than wide; achenes 2.2-3 $\mathrm{mm}$ long.

2. Beaks of perigynia appressed-ascending; spikes oblong-cylindric; achenes obovoid, their sides concave; pistillate scales mostly blunt; style straight below. Expected (C. typhina)

2. Beaks of perigynia widely radiating; spikes oval or oblong-oval; achenes linear-oval, their sides almost flat; pistillate scales acute to short-awned; style strongly curved below . ...................... . squarrosa 
Java Farm. One station: beside old entrance road near junction with road to springhouse. Hectare 3408. Associate species: Juncus tenuis, Agropyron repens, Verbascum blattaria.

Ivy Neck. Infrequent; at edge of woods near drainage of Cheston Creek. Canopy of Liquidambar styraciflua, Platanus occidentalis, Diospyros virginiana. Higman 797, 859, 1020.

C. squarrosa L.

Squarrosa Sedge

(Sedge)

Ivy Neck. One station: west bank of upper Cheston Creek estuary. Canopy of Quercus velutina, Acer rubrum, Juglans nigra, Liquidambar styraciflua. Higman 744.

\section{Section Bracteosae}

I. Sheaths loose, mottled with green and white, usually septate-nodulose dorsally; leaf-blades 4.5-8 mm wide. Expected (C. aggregata, C. sparganioides).

I. Sheaths tight, not mottled or inconspicuously so, nor septate-nodulose dorsally (except sometimes in C. leavenworthii); leaf-blades I-4.5 mm wide.

2. Perigynia distended and spongy at the base, usually wide-spreading or reflexed at maturity.

3. Beaks of perigynia smooth, scarcely exceeding the acuminate, deciduous scales. Expected (C. retroflexa).

3. Beaks of perigynia minutely serrulate, much exceeding the obtuse or somewhat acute, persistent scales.

4. Stigmas long, slender, seldom twisted, light reddish; perigynium tapering into the beak; leaf-blades $\mathrm{I}-2 \mathrm{~mm}$ wide . . . . . . . . . C. rosea

4. Stigmas short, stout, strongly twisted or contorted, deep red; perigynium abruptly contracted into the beak.

5. Leaf-blades (I.5) 2.5 (3) $\mathrm{mm}$ wide; perigynia 3.3-4.5 $\mathrm{mm}$ long, 6-20

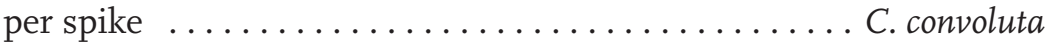

5. Leaf-blades I (I.75) $\mathrm{mm}$ wide; perigynia 2.3-3 $\mathrm{mm}$ long, 2-6 per spike. Expected (C. radiata).

2. Perigynia not distended and spongy at the base, mostly ascending.

6. Inflorescence ovoid or oblong-ovoid; spikes densely capitate.

7. Scales much shorter than bodies of the perigynia.

8. Perigynia broadest below the middle, round-tapering at the base, with raised margins ventrally; beaks long, serrulate....... C. cephalophora

8. Perigynia broadest at the truncate-cordate base, flat ventrally; beaks short, smooth. Expected (C. leavenworthii). 
7. Scales slightly shorter to longer than bodies of the perigynia.

9. Culms lax; perigynia faintly nerved dorsally. Expected (C. mesochorea).

9. Culms stiff, erect; perigynia usually strongly nerved or ribbed dorsally; stigmas long, slender .......... C. muhlenbergia, var. enervis

6. Inflorescence oblong or linear-oblong to elongate and interrupted; spikes not capitate.

IO. Spikes all aggregated; inflorescence I. $5-4 \mathrm{~cm}$ long; sheaths concave or convex at the mouth.

II. Scales reddish-brown to purplish-red; perigynia few nerved dorsally; sheaths not thickened at the mouth. Expected (C. spicata).

II. Scales greenish; perigynia strongly ribbed dorsally; sheaths thickened at the mouth.

I2. Perigynia strongly nerved and low convex ventrally, the margins usually slightly raised. Expected (C. muhlenbergii).

I2. Perigynia nerveless or basally nerved ventrally, the margins not raised at maturity ........ C. muhlenbergii, var. enervis

Io. Spikes not aggregated, the lower ones separate; sheaths truncate and thickened at the mouth. Expected (C. virens).

Carex rosea Schkuhr ex Willd.

Rosy Sedge

(Carex rosea Schk.)

(Sedge)

Java Farm. Infrequent; two stations: Along old entrance road under canopy of mature Fagus grandifolia, Quercus alba, Liriodendron tulipifera, etc. Also at summit of south-facing slope overlooking Fox Creek salt marsh, in small depression: canopy of Robinia pseudoacacia and Ulmus americana; ground cover of Lonicera japonica. Higman 676, 765 .

C. rosea Schkuhr ex Willd.

(C. convoluta Mack. (C. rosea Schk.))

Rosy Sedge

(Sedge)

Java Farm. One station: mature forest west of Muddy Creek, near old entrance road. Hectare 2474. Canopy of Fagus grandifolia, Liriodendron tulipifera, Quercus alba, Acer rubrum, etc. Higman Iog8.

C. cephalophora Muhl. ex Willd.

Oval-leaf Sedge

(C. cephalophora Muhl.?)

(Sedge)

Java Farm. One station: shore of Muddy Creek upstream from old entrance road. Hectare 2467. Canopy of Fagus grandifolia, Platanus occidentalis, Liriodendron tulipifera; Understory of Cornus florida, Lindera benzoin. Associate species: Carex blanda, Duchesnea indica, Glyceria striata, Onoclea sensibilis. Higman Iog6. 
Java Farm. Infrequent, along new entrance road. (Collected before resurfacing. Associate species: Carex blanda, Melilotus alba, Ranunculus bulbosus, Vicia sativa, Lepidium campestre.) Higman 623, 1094, 1095.

Section Multiflorae

Carex valpinoidea Michx.

Fox Sedge

(Sedge)

Ivy Neck. One station: strip of moist woods at source of Cheston Creek drainage. Hectare 4752. Canopy of Platanus occidentalis, Diospyros virginiana, Robinia pseudoacacia, etc. Associate species: Carex crinita, Glyceria striata, Poa compressa. Higman 736.

Section Ovales

I. Wing of perigynium abruptly narrowed near middle of body; sterile culms strongly developed, their leaf-blades numerous, spreading, not clustered at the apex.

2. Tips of perigynia appressed or ascending; perigynia thin, barely distended over the achenes $\ldots \ldots \ldots \ldots \ldots \ldots \ldots \ldots \ldots \ldots$. tribuloides

2. Tips of perigynia recurved or spreading; perigynia firm, obviously distended over the achenes. Expected (C. cristatella).

I. Wing of perigynium not narrowed near middle of body; sterile culms often poorly developed, their leaf-blades erect or ascending, clustered toward the apex. 3. Perigynia obovate, widest near the top. Expected (C. longii, C. albolutescens, C. alata).

3. Perigynia not obovate, widest near middle or base.

4. Leaf-sheaths green and strongly nerved ventrally nearly to the mouth. Expected (C. hormathodes).

4. Leaf-sheaths strongly white-hyaline ventrally.

5. Perigynia lanceolate to narrowly ovate-lanceolate, 3 to 4 times as long as

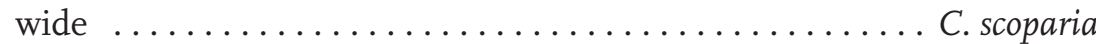

5. Perigynia ovate-lanceolate or broader, at most twice as long as wide. Expected (C. tenera, C. normalis, C. festucaea, C. molesta).

Carex tribuloides Wahlenb.

Blunt Broom Sedge

(Carex tribuloides Wahl.)

(Sedge)

Java Farm. One station: beside Fox Point Road, approx. hectare 3523. Associate species: Eragrostis spectabilis, Dianthus armeria, Juncus tenuis, Euphorbia maculata, etc. Higman 733,893 .

Ivy Neck. See next page. 
Ivy Neck. One station: moist woods at head of Cheston Creek drainage. Hectare 4752. Canopy of Platanus occidentalis, Diospyros virginiana, Robinia pseudoacacia, etc. Associate species: Carex crinita, Glyceria striata, Poa compressa, Carex vulpinoidea. Higman 733,893 .

C. scoparia Schkuhr ex Willd.

Broom Sedge

(C. scoparia Schk.)

(Sedge)

Ivy Neck. One station: north end of grassy abandoned field near Cheston Creek drainage. Hectare 4762. Associate species: Carex frankii, Juncus effusus, Juncus dichotomus. Higman 879.

Section Cryptocarpae

Carex crinita Lam.

Fringed Sedge

(Carex crinita Lam.?)

(Sedge)

Ivy Neck. One station: moist woods at source of Cheston Creek drainage. Hectare 4752. Canopy of Platanus occidentalis, Diospyros virginiana, Robinia pseudoacacia, etc. Associate species: Carex vulpinoidea, Carex tribuloides, Glyceria striata, Poa compressa. Higman 729.

Section Montanae

Carex albicans Willd. ex Spreng. var. emmonsii Emmons' sedge (Dewey ex Torr.) J. Rettig

(Sedge) (Carex emmonsii Dewey)

Java Farm. One station: low, mossy bluffs at head of short, broad valley tributary to Muddy Creek, south of Fox Point Road. Hectare 353I. Canopy of mature Quercus alba, Quercus velutina, Carya tomentosa, and Pinus virginiana. Associate species: Luzula echinata, Carex digitalis. Higman 6I4, 6I5.

Section Laxiflorae

I. Bract-sheaths, base of culms, and staminate scales strongly red-tinged. Expected (C. plantaginea).

I. Bract-sheaths not red-tinged, base of culms rarely so; staminate scales greenish white to dull reddish brown.

2. Perigynia sharply triangular, short-tapering at base, closely 35-50-nerved.

3. Spikes erect, nearly sessile; leaf-blades smooth except on margins, the larger $\mathrm{i2}-25 \mathrm{~mm}$ wide, those of fertile culms much smaller than those of the sterile. Expected (C. platyphylla).

3. Spikes drooping on filiform peduncles; leaf blades hispidulous on the veins, 2-I2 $\mathrm{mm}$ wide, those of the fertile culms moderately smaller than those of the sterile. 
4. Staminate spikes sessile or subsessile, inconspicuous; pistillate ones close together; lowest bract exceeding the inflorescence. Expected (C. abscondita).

4. Staminate spikes peduncled, conspicuous; pistillate ones scattered; lowest bract not exceeding the inflorescence.

5. Pistillate spikes without a staminate flower at the base; leaf-blades $2-5 \mathrm{~mm}$ wide, erect, green $\ldots \ldots \ldots \ldots \ldots \ldots \ldots$. digitalis

5. Pistillate spikes with I or 2 staminate flowers at the base; leaf-blades 5-I2 mm wide, weak, glaucous-green. Expected (C. laxiculmis).

2. Perigynia obtusely triangular at least below, long-tapering at the base.

6 . Bract-sheaths entire or slightly serrulate on the edges. Expected (C. styloflexa, C. laxiflora).

6. Bract-sheaths strongly serrulate on the edges.

7. Sterile shoots reduced to tufts of leaves, not forming culms; leaf-blades 7-30 $\mathrm{mm}$ wide; pistillate scales broadly obovate-orbicular. Expected (C. albursina).

7. Sterile shoots forming conspicuous culms; leaf-blades 3-I2 $\mathrm{mm}$ wide; pistillate scales mucronate to long-awned.

8. Culms not red-tinged at base; lower pistillate spikes not on long capillary peduncles; staminate scales usually greenish white or slightly reddish brown; staminate spike sessile or very short-peduncled; perigynia obovoid, $3-4 \mathrm{~mm}$ long .................. C. blanda

8. Culms red-tinged at base; lower pistillate spikes on long capillary peduncles; staminate scales strongly tinged with reddish brown; staminate spike long-peduncled; perigynia broadly obovoid, $2.5-3.2 \mathrm{~mm}$ long. Expected (C. gracilescens).

Carex digitalis Willd.

Slender Woodland Sedge

(Sedge)

Java Farm. One station: low, mossy bluffs at head of short, broad valley tributary to Muddy Creek, south of Fox Point Road. Hectare 3531. Canopy of mature Quercus alba, Quercus velutina, Carya tomentosa, and Pinus virginiana. Associate species: Luzula echinata, Carex emmonsii. Higman II29.

C. blanda Dewey

Eastern Woodland Sedge

(Sedge)

Java Farm. One station: moist wooded bank of Muddy Creek, upstream from culvert of old entrance road. Hectare 2467. Canopy of Platanus occidentalis, Fagus grandifolia, Liriodendron tulipifera, etc.; understory of Cornus florida, Lindera benzoin. Associate species: Carex cephalophora, Onoclea sensibilis, Duchesnea indica, Glyceria striata.

Ivy Neck. One station: east shore of Scaffold Creek. Canopy of Quercus alba, Quercus velutina, Carya tomentosa. Understory of Viburnum prunifolium. Higman 546, 786. 
Java Farm. Moderately abundant under mature canopy of Fagus grandifolia, Quercus alba, Quercus velutina, Liriodendron tulipifera, Carya tomentosa, and Liquidambar styraciflua. Collected at shoulders of old Muddy Creek Road, near old entrance road, and on slopes over-looking Muddy Creek and its upper tidal portion. Higman 698, 7I2, 987, 992.

Section Vesicariae

Carex lurida Wahlenb.

Shallow Sedge

(Carex lurida Wahl.)

(Sedge)

Java Farm. Infrequent along tributary streams of Muddy Creek (tidal portion). South-facing slope. Canopy of Fagus grandifolia, Quercus velutina, Carya tomentosa, and Liriodendron tulipifera. Associate species: Saururus cernuus.

Ivy Neck. Infrequent, in salt marsh at head of Cheston Creek estuary. Associate species: Typha latifolia, Scirpus americana. Higman 399, 738, 986.

Species of Carex in Expected Sections

Phyllostachyae: C. willdenowii Schkuhr ex Willd. (C. willdenowii Schkuhr), C.jamesii Schwein.

Polytrichoideae: C. leptalea Wahlenb. (C. leptalea Muhl.)

Arenariae: C. arenaria L.

Divisae: C. divisa Huds.

Vulpinae: C. stipata Muhl. ex Willd. (C. stipata Muhl.), C. stipata Muhl. ex Willd. var. maxima Chapm. (C. stipata v. maxima Chapm.), C. laevivaginata (Kük.) Mack. (C. laeyivaginata (Kukenth.) Mack.)

Paniculatae: C. decomposita Muhl.

Deweyanae: C. bromoides Schkuhr ex Willd. (C. bromoides Schkuhr)

Stellulatae: C. seorsa Howe, C. interior L.H. Bailey (C. interior Bailey), C. atlantica L.H. Bailey subsp. capillacea (L.H. Bailey) Reznicek (C. howei Mack.), C. atlantica L.H. Bailey subsp. atlantica (C. incomperta Bickn.), C. atlantica L.H. Bailey (C. atlantica Bailey)

Heleonastes: C. canescens L. subsp. disjuncta (Fernald) Toivonen (C. canescens L. var. disjuncta Fern.)

Acutae: C. emoryi Dewey, C. stricta Lam., C. stricta Lam. (C. stricta var. strictior (Dewey) Carey), C. torta Boott ex Tuck. (C. torta Boott)

Paludosae: C. lacustris Willd. (C. riparia var. lacustris (Kukenth.) Mack.)

Triquetrae: C. hirtifolia Mack.

Hirtae: C. pellita Muhl. ex Willd. (C. lanuginosa Michx.), C. hirta L., C. vestita Willd. 
Lupulinae: C. grayi Carey (C. grayii Carey), C. intumescens Rudge, C. louisianica

L.H. Bailey (C. louisianica Bailey), C. lupulina Muhl. ex Willd. (C. lupulina Muhl.), C. lupuliformis Sartwell ex Dewey (C. lupuliformis Sartw.)

Collinsiae: C. collinsii Nutt.

Folliculatae: C. folliculata L.

Pseudo-cyperi: C. hystericina Muhl. ex Willd. (C. hystricina Muhl.), C. comosa Boott Shortianae: C. shortiana Dewey

Atratae: C. buxbaumii Wahlenb. (C. buxbuamii Wahl.)

Gracillimae: C.gracillima Schwein., C. prasina Wahlenb. (C. prasina Wahl.), C. davisii Schwein \& Torr.

Sylvaticae: C. venusta Dewey var. minor Boeckeler (C. oblita Steud.), C. debilis Michx., C. debilis Michx. var. pubera A. Gray (C. allegheniensis Mack.)

Extensae: C. viridula Michx.

Granulares: C. granularis Muhl. ex Willd. (C. haleana Olney), C. granularis Muhl. ex Willd. (C. granularis Muhl.)

Oligocarpae: C. oligocarpa Schkuhr ex Willd. (C. oligocarpa Schkuhr), C. hitchcockiana Dewey

Griseae: C. amphibola Steud., C. grisea Wahlenb. (C. grisea Wahl.), C. glaucodea

Tuck. ex Olney (C. glaucodea Tuckerm.)

Paniceae: C. woodii Dewey

\section{Cyperus \\ (from O’Neill, I94I)}

I. Achene lenticular, flat with an edge facing the rachilla; styles bifid; spikelets very flat.

2. Spikelets I.5 to $2 \mathrm{~mm}$ long, I-flowered, congested into a solitary cluster of dense, sessile heads. Expected (C. tenuifolius).

2. Spikelets 3 to $20 \mathrm{~mm}$ long, 3- to 40 -flowered, digitate in an umbel-like panicle. 3. Achene suborbicular, black, transversely wrinkled. (Also, spikelets yellow, 2-2.5 mm wide.) Expected (C. flavescens).

3. Achene obovate to oblong-obovate, purplish-brown, not wrinkled.

4. Spikelets I to I.5 mm wide. Expected (C. polystachyos var. texensis).

4. Spikelets 2 to $3.5 \mathrm{~mm}$ wide.

5. Rachilla winged, the wings forming a collar 0.2 to $0.3 \mathrm{~mm}$ wide at the base of the achene; salt marsh habitat......... C. filicinus

5. Rachilla wingless; freshwater marsh habitat. Expected (C. rivularis).

I. Achene 3-angled; styles trifid; spikelets thicker in the center.

6. Rachilla readily disarticulating into I-fruited joints. Expected (C. odoratus).

6. Rachilla not articulated or articulating only at the base.

7. Rachilla wingless; stamens I or 2 ( 3 in C. filicinus).

8. Glumes long-awned, conspicuously 9- to I3-nerved; plants scented. Expected (C. inflexus).

8. Glumes obtuse to mucronate, 3-nerved; plants odorless. 
9. Glumes 3-nerved; stamens I or 2. Expected (C. pseudovegetus, C. fuscus).

7. Rachilla winged; stamens 3 .

Io. Spikelets not all deflexed, the uppermost ascending or spreading, 3- to 40-flowered (sometimes 2-flowered in C. ovularis).

II. Achenes 0.8-I mm long; glumes 3- to 7-nerved. Expected (C. dentatus, C. erythrorhizos).

II. Achenes I.3-3 mm long; glumes 7- to many-nerved.

I2. Achenes 2.2-3 $\mathrm{mm}$ long, 3 to 5 times as long as wide; glumes 4-5.5 mm long. Expected (C. refractus, C. lancastriensis).

I2. Achenes I.3-2 $\mathrm{mm}$ long (rarely $2.2 \mathrm{~mm}$ in $C$. ovularis), ovoid to oblong; glumes I.5-4 $\mathrm{mm}$ long (rarely $4.5 \mathrm{~mm}$ in C. strigosus).

I3. Spikelets 2- to 3-flowered, densely congested into subglobose heads at the ends of the rays; achenes I.8-2.2 $\mathrm{mm}$ long ......................... ovularis

I3. Spikelets 5- to 40-flowered, not in subglobose heads; achenes I.3-2 $\mathrm{mm}$ long.

I4. Spikelets nearly flat; achenes linear, I.5-I.8 mm long; glumes $3.7-4.5 \mathrm{~mm}$ long ........... C. strigosus

I4. Spikelets subterete or sub-quadrangular; achenes ovoid to oblong, 0.5-I.2 mm long; glumes I.5-3.5 mm long. Expected (C. esculentus, C. grayii).

Io. Spikelets all deflexed at maturity, I- or 2-flowered. Expected (C. retrofractus, C. dipsaciformis).

Java Farm. Infrequent. One station in Hog Island salt marsh on inland side. Hectare 3572. Associate species: Panicum virgatum, Juncus dichotomus, Osmunda cinnamomea, Distichlis spicata.

Also one station on Fox Point Road; associate species: Festuca myuros, Plantago aristata, Lepidium virginicum, Juncus tenuis, Digitaria ischaemum, Cyperus ovularis. Higman 438, 804.

C. echinatus (L.) Alph. Wood

(C. ovularis (Michx.) Torr.)

\section{Globe Flatsedge}

Java Farm. Infrequent along Fox Point Road. Associate species same as for C. filicinus.

Ivy Neck. One station: marshy north end of grassy abandoned field on Scaffold Peninsula. Hectare 4762. Associate species: Juncus effusus, Juncus dichotomus, Phleum pratense, Trifolium pratense, Agrostis alba, Carex frankii. Higman I7, 490, I099. 
Java Farm and Ivy Neck. Moderately abundant in a variety of habitats; seems to prefer unshaded sites whether dry, moist, or slightly saline. Collected at old entrance gate of Java Farm, Spartina patens community of Fox Creek salt marsh, old field south of Fox Point Road (associate species: Lespedeza cuneata), upstream limit of salt marsh on Muddy Creek, and border of cornfield on Cheston Peninsula. Higman 97, I24, I54, 256, 1006, I032.

\section{Eleocharis}

I. Spikelet not thicker than the culm; scales persistent. (Also, culm quadrangular, not septate). Expected (E. quadrangulata).

I. Spikelet much thicker than the culm; scales deciduous.

2. Achenes lenticular or biconvex, styles 2-cleft.

3. Uppermost sheaths white, scarious and loose at the mouth; dwarfed annual usually less than Io $\mathrm{cm}$ tall. Expected (E. olivacea).

3. Uppermost sheaths greenish or reddish, opaque, tight-fitting at the mouth; plant IO-I30 cm tall. (Also, achenes whitish to tawny or brown at maturity).

4. Tubercle only slightly constricted from the achene; plants densely tufted annuals without long, firm rhizomes or stolons.

5. Perianth bristles wanting or rarely as long as the achene; tubercle less than I/4 as high as achene. Expected (E. engelmanni).

5. Perianth bristles longer than the achene and tubercle; tubercle $\mathrm{I} / 3$ to I 2 as high as achene. .................... obtusa

4. Tubercle deeply constricted from the achene; plants not densely tufted, with long, firm rhizomes or stolons.

6. Basal scales of spikelet 2 or 3, the lowest not encircling the culm. Expected (E. palustris).

6. Basal scale I, encircling the culm.

7. Spikelet closely many-flowered; scales broadly obtuse or rounded, opaque, appressed. Expected (E. calva).

7. Spikelet loosely 5- to 30-flowered; scales acute or subobtuse, lustrous, loosely ascending ............... E. halophila

2. Achenes trigonous, styles 3-cleft.

8. Achenes with shallow to deep honeycomb reticulation, or with prominent longitudinal ridges. Expected (E. acicularis, E. tortilis).

8. Achenes smooth or minutely roughened.

9. Perianth bristles longer than the achene and tubercle; plant a tufted annual. (Also, mouth of sheath oblique; spikelets lanceolate; achene smooth $\ldots \ldots \ldots \ldots \ldots \ldots \ldots \ldots \ldots \ldots \ldots \ldots \ldots$ intermedia

9. Perianth bristles shorter than the achene; plant perennial with long, slender rhizomes. (Also, culms flat, scales acuminate or attenuate). Expected (E. compressa). 
Eleocharis obtusa (Willd.) Schult.

Blunt Spikerush

(Eleocharis obtusa (Willd.) Schultes? (immature))

(Spikerush)

Ivy Neck. One station: abundant in salt marsh at head of Cheston Creek estuary. Hectare 4753. Associate species: Typha latifolia, Scirpus validus. Higman 74I.

\section{E. halophila (Fernald \& Brack.) Fernald \& Brack. Saltmarsh Spikerush (E. halophila L.) \\ (Spikerush)}

Java Farm. Two stations in salt marshes: Hog Island marsh (pure stand north of Hog Island, hectare 3582) and Typha latifolia marsh at junction of forks of Muddy Creek (hectare 3436). Higman 298, 677.

E. intermedia Schult.

Matted Spikerush

(E. intermedia L.)

(Spikerush)

Ivy Neck. Two stations near drainage to Cheston Creek estuary: north end of grassy abandoned field on Scaffold Peninsula (hectare 4762) and beside artificial pond above Cheston Peninsula (Murray property) Higman 88I, 883, 994.

\section{Schoenoplectus, Scirpus}

I. Involucre subtending the inflorescence consisting of a single bract resembling a continuation of the culm; culms leafless or basally leaved.

2. Inflorescence without long branches, either a spike, a spikelet, or a cluster of spikelets; culms 3-angled, or if terete less than $3 \mathrm{~mm}$ thick at the base.

3. Plants tufted, annual, without rhizomes; mature achenes black or blackish. Expected (S. smithii, S. purshianus).

3. Plants mostly solitary or scattered, perennial, with long rhizomes; mature achenes whitish, drab, or brown. (Also, rhizome hard; upper leaf sheaths closed; spikelets solitary or clustered, ovoid, reddish-brown to dark brown; scales ovate to orbicular, the margins irregular).

4. Involucre linear, 2-I5 cm long, acute; upper sheath concave but not notched at orifice, with a long, linear, sharp-pointed blade ........

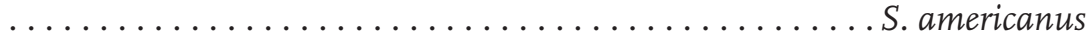

4. Involucre lance-triangular, I-3.5 cm long, blunt; upper sheath V-notched at orifice, with a lanceolate, blunt-pointed blade 8-I5 mm long. Expected (S. olneyi).

2. Inflorescence with long branches; culms terete, 3 to $20 \mathrm{~mm}$ thick at the base. (Also, style 2-cleft; achenes plano-convex; scales reddish to deep brown).

5. Scales glabrous, not gummy or spotted, the green midrib projecting as an awn; basal sheath membranaceous, with scarious margin . S. validus, var. creber

5. Scales pubescent, with many gummy dots, midrib mucronate; basal sheath firm, with fibrillose margin. Expected (S. acutus). 
I. Involucre of 2 or more flat leaves; culms leafy.

6. Culms sharply 3-angled, solitary or scattered; spikelets I to $5 \mathrm{~cm}$ long, 5 to II mm thick. (Also, midribs of scales awned).

7. Achenes equilaterally 3-angled; freshwater marsh habitat. Expected (S. fluviatilis).

7. Achenes plano-convex to obtusely 3-angled; salt marsh habitat. (Also, spikelets reddish-brown, blunt or rounded at apex; ligule scarious). . . . . $\ldots \ldots \ldots \ldots \ldots \ldots \ldots \ldots \ldots \ldots$. robustus

6. Culms obtusely angled, or sometimes sharply at summit; spikelets 2 to I5 $\mathrm{mm}$ long, I to $3 \mathrm{~mm}$ thick. (Also, spikelets 35 to over Ioo, in decompound umbelliform panicles).

8. Bristles retrorsely barbed; culms solitary or few; stolons thick and scaly.

9. Bristles barbed nearly to base; lower sheaths reddish. Expected (S. expansus).

9. Bristles barbed only above the middle; all sheaths greenish.

Io. Bristles twice as long as achene; spikelets reddish-brown. Expected (S. polyphyllus).

Io. Bristles not exceeding achene; spikelets pale to dark brown. (Also, scales round) $\ldots \ldots \ldots \ldots \ldots \ldots \ldots \ldots \ldots \ldots \ldots$ S. atrovirens

8. Bristles smooth or with few hairs; culms tufted; stolons absent.

II. Bristles barely exceeding the scales. Expected (S. lineatus).

II. Bristles greatly exceeding scales at maturity. (Also, lateral spikelets in the ultimate fascicles pedicelled, central one sessile; spikelets reddishbrown, ovoid) S. rubricosus

Schoenoplectus americanus (Pers.)

Chairmaker's Bulrush

Volkart ex Schinz \& R. Keller

(Bulrush) (Scirpus americanus Pers.)

Java Farm. Infrequent in Hog Island salt marsh, at shore of Muddy Creek estuary. Associate species: Atriplex patula, Polygonum punctatum, Aster puniceus.

Ivy Neck. One station: beach at Sand Point, on Cheston Peninsula. Associate species: Cakile edentula, Spartina alterniflora. Higman 295, 45I, 937.

\section{S. americanus (Pers.) Volkart ex Schinz \& R. Keller}

Chairmaker's Bulrush (S. americanus Pers. (extreme form))

Ivy Neck. Salt marsh at head of Cheston Creek estuary. Associate species: Typha latifolia, Scirpus validus, Carex lurida, Juncus bufonius, Eleocharis sp. Higman 856. 
Java Farm. Infrequent in Hog Island salt marsh. Associate species: Scirpus americanus, Pluchea camphorata, Distichlis spicata, Panicum virgatum.

Ivy Neck. Moderately abundant in salt marsh at head of Cheston Creek estuary. Co-dominant with Typha latifolia. Less frequent in moist woods of Cheston Creek drainage, under canopy of Platanus occidentalis, Diospyros virginiana, Liquidambar styraciflua. Higman 257, 294, 737.

\section{S. robustus (Pursh) M.T. Strong}

Sturdy Bulrush

(S. robustus Pursh.)

(Bulrush)

Java Farm and Ivy Neck. Infrequent in salt marshes. Collected at Fox Point and at the head of Cheston Creek estuary. Also observed along the tidal portion of Muddy Creek. Associate species: Typha latifolia, Pluchea camphorata, Scirpus americanus, Iva frutescens. Higman 56, 857 .

Scirpus atrovirens Willd.

\section{Green Bulrush}

(Bulrush)

Ivy Neck. One station: moist woods at Cheston Creek drainage. Mature canopy of Platanus occidentalis, Diospyros virginiana, and Liquidambar styraciflua. Associate species: Carex crinita, Glyceria striata, Poa compressa, Eupatorium coelestinum, Carex vulpinoidea. Higman 728.

\section{S. cyperinus (L.) Kunth}

Woolgrass

(S. rubricosus Fern.)

(Bulrush)

Java Farm. Infrequent; stations in the freshwater part of Fox Creek marsh and at the marshy west end of the Phalaris arundinacea meadow north of the main building area. Associate species: Juncus effusus, Polygonum pensylvanicum, Impatiens capensis, Aster praealtus, Salix nigra, Sambucus canadensis. Higman I90 495.

\section{ARACEAE}

Arisaema

I. Lateral leaflets very rounded on lower side, tapering to base on the upper side; tube of spathe $3.5-7.0 \mathrm{~cm}$ long, summit flange $2-8 \mathrm{~mm}$ broad, hood 3-6 cm broad; fruiting head $3-6 \mathrm{~cm}$ long . . . . . . . . . . . atrorubens

I. Lateral leaflets narrowed subequally at base, not especially rounded on the lower side; measurements of other parts usually smaller than above but slightly overlapping $\ldots \ldots \ldots \ldots \ldots \ldots \ldots \ldots \ldots \ldots \ldots \ldots \ldots \ldots \ldots \ldots \ldots \ldots \ldots \ldots \ldots$ triphyllum 
Java Farm. Infrequent; prefers moist, shaded sites. Along old entrance road under canopy of Liquidambar styraciflua, Fagus grandifolia, Quercus alba, Acer rubrum, etc. Associate species: Podophyllum peltatum.

Also along moist terrace of Muddy Creek under canopy of Fraxinus americana and Platanus occidentalis.

Ivy Neck. Moderately abundant in similar habitats, especially source of Scaffold Creek, under canopy of Liquidambar styraciflua, Acer rubrum, Robinia pseudoacacia, etc. Associate species: Viola spp., Podophyllum peltatum, Claytonia virginica. Hig$\operatorname{man} 72,600$.

A. triphyllum (L.) Schott

Jack in the Pulpit

(Small Jack-in-the-pulpit)

Java Farm and Ivy Neck. Habitats and associate species the same as for A. atrorubens above. Higman 544 .

\section{LEMNACEAE}

I. Floating frond flattish, ovate to circular; rootlets present, I to several.

2. Rootlet I; frond I- to 5-nerved, green beneath . . . . . . . . . . Lemna

2. Rootlets 2 or more; frond 4 - to I5-nerved, purple beneath . . . . . . . Spirodela

I. Floating frond ellipsoid to globose (like green rice); rootlets absent. Expected (Wolffia, Wolffiella).

Lemna

Lemna minor L.

Common Duckweed

(Duckweed)

Ivy Neck. Abundant on surface of small cut-off pond near Sand Point on Cheston Peninsula. Hectare 4870. Associate species: Spirodela polyrhiza above. Higman I225.

Spirodela polyrrhiza (L.) Schleid.

Spirodela

(Spirodela polyrhiza (L.) Schleid.)

Common Duckmeat

(Water- flaxseed)

Ivy Neck. Abundant on surface of small, fresh to slightly saline cut-off pond near Sand Point on Cheston Peninsula. Hectare 4872. Associate species: Lemna minor, Rumex verticillatus, Sparganium eurycarpum. Higman I226. 


\section{COMMELINACEAE}

\section{Commelina}

Commelina communis L.

Asiatic Dayflower

(Commelina communis L. var. ludens (Miquel) C. B. Clarke)

(Dayflower)

Java Farm. Infrequent along Old Muddy Creek Road and in ditch at entrance gate. Partly shaded by adjacent canopy of Fagus grandifolia, Quercus alba, and Liriodendron tulipifera. Associate species: Polygonum spp., Oxalis stricta, Galium aparine, Dactylis glomerata, Geranium carolinianum, Sphenopholis nitida, Tovara virginiana, Hieracium scabrum. Higman $35^{2}$.

\section{PONTEDERIACEAE}

\section{Heteranthera}

Heteranthera reniformis Ruiz \& Pav.

(Heteranthera reniformis R. \& P.)

Kidneyleaf Mudplantain

(Mud-plantain)

Ivy Neck. One station: muddy bank of artificial pond at source of Cheston Creek drainage, on Murray farm. Associate species: Gratiola neglecta, Ranunculus sceleratus. Higman 874 .

\section{JUNCACEAE}

I. Leaves and young steins pubescent; capsule I-locular . . . . . . . . Luzula

I. Leaves and young stems glabrous; capsule 3 -locular .......... Juncus

Luzula echinata (Small) F.J. Herm. (Luzula echinata (Small) F. J. Herm.)

\section{Luzula}

Java Farm. Infrequent on moist, south-facing forested slope overlooking upper tidal portion of Muddy Creek, One station adjacent to Typha latifolia salt marsh and one at source of tributary stream.

Canopy of Quercus alba, Q. velutina, Carya tomentosa, Liriodendron tulipifera, etc. Associate species: Carex emmonsii. Higman 7I7, I097.

\section{Juncus}

I. Individual flowers subtended by a pair of bracteoles as well as the bractlet at base of pedicel.

2. Inflorescence terminal, subtended by one or more slender divergent leaves.

3. Leaf sheaths tapering to summit, not auricled; inflorescence at least one quarter height of plant $\ldots \ldots \ldots \ldots \ldots \ldots \ldots \ldots \ldots \ldots \ldots$. bufonius 
3. Leaf sheaths auricled at summit; inflorescence less than one quarter height of plant. (Also, auricles entire; rhizome short, hidden by tussock; tips of sepals not appressed to capsule).

4. Auricles prolonged, scarious, lance-triangular; leaves flat ......J. tenuis 4. Auricles rounded, not lance-triangular; leaves terete or only channeled on upper side ......................... J. dichotomus

2. Inflorescence apparently lateral, the subtending leaf like a continuation of

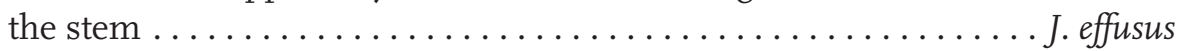

I. Individual flowers subtended only by a bractlet at base of the very short pedicel. 5. Leaves not septate. Expected (J. repens, J. biflorus).

5. Leaves septate. (Also, heads of flowers not spherical).

6 . Heads hemispherical to nearly spherical ............. J. canadensis 6. Heads less than hemispherical ................. J. acuminatus

Juncus bufonius L. Toad Rush (Toad-rush)

Ivy Neck. Principal station in marsh at head of Cheston Creek estuary. Hectare 4753. Co-dominant with Eleocharis palustris. Adjacent to stands of Typha latifolia and Scirpus validus.

Infrequent at forest border of cornfield; there associated with Juncus tenuis. Hig$\operatorname{man} 739,838$.

J. tenuis Willd.

Poverty Rush

(Bog-rush)

Java Farm and Ivy Neck. Moderately abundant on dry, unshaded areas (fields, bluffs overlooking Rhode River near Sand Point, etc.), roadsides, and cracked pavement. Wide variation of associate species. Higman 24, I73, 796, 824, 837, 839 .

\section{J. anthelatus (Wiegand) R.E. Brooks}

(Juncus tenuis, f. anthelatus (Wieg.) F. J. Herm.)

Greater Poverty Rush

Java Farm. One station in broken pavement at Fox Point. Hectare 3557. Canopy of Quercus prinus. Higman 434.

\section{J. dichotomus Elliott}

Forked Rush

(J. dichotomus Ell.)

(Bog-rush)

Java Farm. One station: in Hog Island salt marsh. Hectare 3582. Associate species: Panicum virgatum.

Ivy Neck. One station: marshy north end of abandoned wheat-field. Hectare 4762 . Associate species: Juncus effusus, Carex frankii, Carex scoparia, and Scirpus atrovirens. Higman 304, 749. 
Java Farm. Infrequent at marshy west end of meadow north of main buildings. Hectare 2468. Associate species: Phalaris arundinacea (dominant), Polygonum saggitatum, and Scirpus rubricosus.

Ivy Neck. Infrequent at marshy end of abandoned wheatfield. Hectare 4762. Associate species: Carex scoparis, Eleocharis intermedia. Higman 748, 880.

Java Farm. One station: bank of Muddy Creek above entrance road culvert. Hectare 2467. Open canopy of Platanus occidentalis, Fagus grandifolia, and Liriodendron tulipifera. Understory of Cornus florida and Lindera benzoin. Associate species: Glyceria striata, Panicum clandestinum, and Duchesnea indica. Higman 792.

J. acuminatus Michx.

Tapertip Rush

(Bog-rush)

Ivy Neck. One station: dry bank of artificial pond on upper Cheston Peninsula. Associate species: Echinochloa pungens. Higman 997.

\section{AGAVACEAE, LILIACEAE, SMILACACEAE}

I. Flowers or inflorescence terminal.

2. Flowers solitary. Expected (Lilium, Erythronium).

2. Flowers in clusters.

3. Plant woody, evergreen; leaves rigid, erect, with marginal shreddy fibers. Sandy shore habitat. (Also, flowers white, campanulate, in a panicle).....

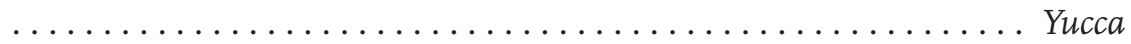

3. Plant herbaceous, not evergreen; leaves not rigid, without marginal shreddy fibers. Inland habitat.

4. Flowers I dm long or more, very showy.

5. Flowering stem arising from fibrous roots; leaves basal ........

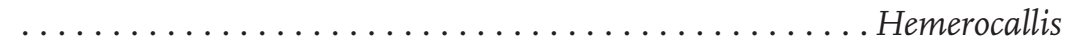

5. Flowering stem arising from a scaly bulb; leaves cauline. Expected (Lilium).

4. Flowers considerably smaller.

6. Flowers in umbels.

7. Flowers white or pink; leaves basal; plant onion-scented ... Allium

7. Flowers greenish yellow; leaves cauline; plant odorless. Expected (Medeola).

6. Flowers in racemes, corymbs, or panicles. 
8. Styles 3. Expected (Amianthium, Stenanthium).

8. Style I, sometimes cleft at tip.

9. Base bulbous; leaves linear, basal; fruit a capsule. (Also, perianth segments white with broad green midribs) ...... Ornithogalum

9. Base not bulbous, arising from long rhizomes; leaves oblong to ovate, cauline; fruit a berry . ............... Smilacina

I. Flowers or inflorescences axillary.

Io. Plant thorny, a woody vine, often with tendrils

Smilax

Io. Plant thornless, herbaceous, without tendrils.

II. Leaves reduced to small scales; plant extremely branched .... Asparagus

II. Leaves normally developed. Expected (Uvularia). See Appendix I.

Yucca

Yucca filamentosa L.

Adam's Needle

Ivy Neck. Infrequent on sandy beach facing Rhode River, well north of Sand Point. Higman I076.

Hemerocallis fulva (L.) L.

Hemerocallis

(Hemerocallis fulva L.)

Orange Daylily

(Day Lily)

Ivy Neck. One station: yard of abandoned house north of entrance road. Hectare 4720. Persistent after cultivation. Higman 961.

$\begin{array}{lr}\text { Allium vineale L. } & \text { Allium } \begin{array}{r}\text { Wild Garlic } \\ \text { (Wild Onion, Field Garlic) }\end{array}\end{array}$

Java Farm and Ivy Neck. Moderately abundant to abundant in old fields and in moist, moderately shaded areas near freshwater marshes and streams. Higman 784 .

Ornithogalum umbellatum L.

Ornithogalum

Java Farm. One station: large field along north border. Hectare 3508. Dense Rhus radicans. Higman II46. 


\section{Maianthemum}

(Smilacina)

Maianthemum racemosum (L.) Link

ssp. racemosum

(Smilacina racemosa (L.) Desf.)

Feathery False Lily of the Valley

(False Solomon's Seal)

Java Farm. Infrequent to moderately abundant in mature forest beside old entrance road. Canopy of Fagus grandifolia, Quercus alba, Liriodendron tulipifera, etc. Associate species: Dentaria laciniata, Dentaria heterophylla, Claytonia virginica. Higman 4I2, 668.

\section{Smilax}

I. Leaves whitened or glaucous beneath; berries blue ............ S. glauca

I. Leaves green beneath; berries blue-black .............. rotundifolia

\section{Smilax glauca Walter}

Cat Greenbrier

(Smilax glauca Walt.)

Java Farm. Infrequent on Fox Point. Canopy of mature Quercus prinus. Associate species: Baptisia tinctoria, Epigaea repens, Gaylussacia baccata. Higman I072.

\section{S. rotundifolia $\mathrm{L}$.}

Roundleaf Greenbrier

(Greenbriar)

Java Farm and Ivy Neck. Abundant on the landward borders of salt marshes, often forming a dense barrier. Apparently delimits the saline and freshwater parts of marshes. Associate species: Rosa palustris, Typha latifolia, Hibiscus palustris. Higman 269.

Asparagus officinalis L.

$$
\text { Asparagus }
$$

\section{Garden Asparagus}

(Asparagus)

Java Farm. One station: lawn at main building area. Hectare 2580. Associate species: Medicago lupulina, Melilotus alba, Allium vineale.

Ivy Neck. Infrequent on sandbars, landward borders of salt marshes, and muddy shores of Cheston Creek and Scaffold Creek estuaries. Higman 740. 


\section{DIOSCOREACEAE}

Dioscorea

Dioscorea villosa L.

Wild Yam

(Yam)

Java Farm. One station: beside old entrance road. Hectare 2474. Mature canopy of Fagus grandifolia, Quercus alba, Liriodendron tulipifera, etc. Associate species: Claytonia virginica, Dentaria heterophilla, Smilacina racemosa, Viola papilionacea. Higman 699.

\section{LILIACEAE \\ (AMARYLLIDACEAE)}

I. Perianth and fruit glabrous; base a truncated bulb. (Also, flowers loosely spreading or nodding; perianth with a slender tube and campanulate or cup-like crown and a spreading $\operatorname{limb}) \ldots \ldots \ldots \ldots \ldots \ldots \ldots \ldots \ldots \ldots \ldots \ldots$ Narcissus

I. Perianth pilose on the outside; base a corm, rhizome, or tuber. (Also, tube of perianth covering ovary and perianth segments converging to form a beak over the fruit; leaves linear, usually pubescent $\ldots \ldots \ldots \ldots \ldots \ldots$ Hypoxis

\section{Narcissus}

I. Flowers yellow; crown about as long as perianth-segments . . . N. pseudonarcissus

I. Flowers white; crown much shorter than perianth-segments . . . . N. poeticus

Narcissus pseudonarcissus L.

Daffodil

Java Farm. One station: steep east-facing slope of ridge near north border, north of new entrance road (yard of old house). Hectare 2562. Canopy of mature Juglans nigra. Persistent after cultivation. Higman $55^{2}$.

N. pseudonarcissus L.

Daffodil

(N. pseudonarcissus L., var.?)

Ivy Neck. One station: yard of old house between entrance road and source of Scaffold Creek drainage. Hectare 4658. Persistent after cultivation. Higman 586.

N. poeticus L.

Poet's narcissus

(Poets' Narcissus)

Java Farm. One station: summit of steep ridge near north border, north of new entrance road (yard of old house). Hectare 2562. Canopy of mature Juglans nigra. Persistent after cultivation. Higman 6oI. 
Java Farm. One station: bank of tributary of Muddy Creek, near old Muddy Creek Road. Hectare 249I. Canopy of mature Fagus grandifolia. Associate species: Claytonia virginica, Smilacina racemosa. Higman 670.

\section{IRIDACEAE}

Sisyrinchium

Sisyrinchium angustifolium Mill.

Narrowleaf Blue-eyed Grass

(Blue-eyed Grass)

Java Farm. One station: mound of charcoal in yard of burned house on road to springhouse. Hectare 34I7. Partial canopy of Rhus typhina. Associate species: Trifolium repens, Lepidium virginicum. Higman 709.

\section{ORCHIDACEAE}

I. Inflorescence a terminal spike; stem with narrow, clasping leaves. (Also, basal leaves fleshy and evergreen, with white veins) ............... Goodyera

I. Inflorescence a raceme or of one terminal flower; flowers borne on a scape.

2. Flowers remotely racemose, exceeded by leafy bracts; lips of corolla pink and

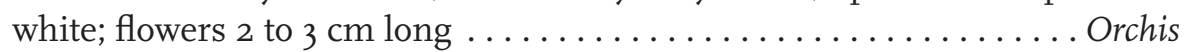

2. Flowers solitary, terminal, without leafy bracts; lip of corolla pink or roseate, inflated, drooping, 3.5 to $7 \mathrm{~cm}$ long ..................... Cypripedium

Goodyera pubescens (Willd.) R. Br.

\section{Goodyeta pubescens (Willd) R. Br.}

\section{Goodyera}

Java Farm. Infrequent in mature forest west of Muddy Creek. Canopy of Fagus grandifolia, Liriodendron tulipifera, Quercus alba. Also one station in mature forest at Fox Point. Canopy of Quercus alba, Quercus prinus. Higman 250.

\section{Galearis spectabilis (L.) Raf.}

\section{Galearis}

(Orchis)

(Orchis spectabilis L.)

Showy Orchid (Showy Orchis)

Java Farm. One station: mature forest west of Muddy Creek, near old entrance road. Canopy of Fagus grandifolia, Quercus alba, and Liriodendron tulipifera. Associate specie: Dentaria heterophilla, Claytonia virginica, Smilacina racemosa. Rare species. Higman 654 . 
Java Farm. Infrequent: two stations under canopy of mature Fagus grandifolia and Quercus alba. One station near apex of triangular area west of old Muddy Creek Road, near freshwater marsh. One on northeast-facing slope between old entrance road and main building area, north of water tank. Rare species. Higman 476, 669.

\section{SAURURACEAE}

\section{Saururus}

Saururus cernuus L.

Lizard's Tail

(Lizard's-tail)

Java Farm. Infrequent, in valleys of tributaries to Muddy Creek. Canopy of Quercus alba, Fagus grandifolia, etc. Higman 397, 985.

\section{SALICACEAE}

I. Leaves linear to lanceolate, acute; aments ascending or divergent . . . . . . Salix

I. Leaves broadly ovate or triangular, acuminate; aments arching or drooping ...

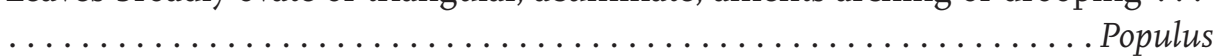

\section{Salix}

The species included in this key are those from the Washington-Baltimore flora (Hermann, I946) which the available literature does not report as restricted to the Piedmont. Of these seven species, three (S. nigra, S. sericea, and S. tristis) are represented from the Maryland Coastal Plain in the U. S. National Herbarium. Only S. nigra has been found at the Chesapeake Bay Center to date. This key should facilitate the search for additional species there.

I. Leaves entire or sparingly undulate-crenate, narrowly oblanceolate to obovate; plant a shrub. (Also, aments preceding the leaves; staminate and pistillate flowers each with one basal gland.) Expected (S. humilis, S. tristis).

I. Leaves closely serrulate, linear-lanceolate to broadly lanceolate; plant a tree (shrubby in S. sericea). (Also, scales of ament pubescent.)

2. Aments sessile, preceding the leaves; scales blackish, persistent; capsules silky-puberulent; staminate flowers each with one basal gland. (Also, leaves acuminate, glaucous and silky beneath; stamens 2.) Expected (S. sericea).

2. Aments on leafy peduncles, accompanying the leaves; scales yellow, deciduous; capsules glabrous; staminate flowers each with two basal glands.

3. Petioles not glandular above at apex; stamens 3 to 8; pistillate flowers whorled. (Also, leaves long-acuminate). 
4. Leaves glaucous beneath. (Also, leaves somewhat pubescent, especially on midribs). Expected (S. caroliniana).

4. Leaves green beneath. ...................... nigra

3. Petioles glandular above at apex; stamens usually 2; pistillate flowers spirally arranged.

5. Leaves glabrous at maturity, with 4 to 6 teeth per $\mathrm{cm}$ of margin; capsules short-pedicelled. Expected (S. fragilis).

5. Leaves silky-pubescent, with 7 to 9 teeth per $\mathrm{cm}$ of margin; capsules sessile. Expected (S. alba).

Salix nigra Marshal

Black Willow

(Salix nigra Marsh.)

Java Farm. Infrequent, in freshwater portion of marsh at mouth of Fox Creek drainage and at the landward margin of small marsh on the north side of Fox Point peninsula. Hectares 3526, 3557. Associate species: Rosa palustris.

Ivy Neck. Infrequent, in moist wooded strip at source of Cheston Creek estuary. Hectare 4733. Canopy of Diospyros virginiana, Robinia pseudoacacia, Platanus occidentalis, etc. Higman 465, 493, 525, 589 .

\section{Populus}

I. Leaves ovate, short-acuminate, wooly when young but glabrate at maturity; teeth

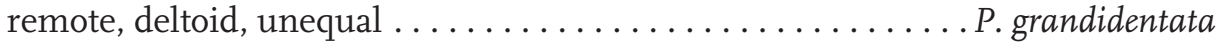

I. Leaves triangular, acuminate, glabrous at all ages; teeth numerous, equal. ....

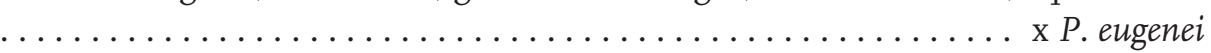

Populus grandidentata Michx.

Bigtooth Aspen

(Big-toothed Aspen)

Java Farm. One station: rubbish pile on north side of headquarters, near big steel shed. Hectare 2489. Associate species: Paulownia tomentosa, Celtis occidentalis. Higman 9I3.

P. x canadensis Moench (pro sp.) [deltoides x nigra] Carolina Poplar (x P. eugenei Simon-Lewis)

(P. deltoides Marsh, x P. nigra L. var. italica Muench.)

(x P. canadensis Moench var. eugenei (Simon-Lewis) Schelle))

Ivy Neck. One station: two large trees in yard of old house near entrance road. Hectare 4658. Higman Io89, II42. 


\section{MYRICACEAE}

\section{Morella}

(Myrica)

\section{Morella cerifera (L.) Small}

(Myrica cerifera L.)

Wax Myrtle

Java Farm and Ivy Neck. Moderately abundant along shores of estuaries, under a variety of hardwood canopies. Infrequent on sand-bars and at margins of salt marshes. Higman 572.

\section{JUGLANDACEAE}

I. Leaves with Io to I7 leaflets; nut with fleshy indehiscent husk and irregularly furrowed shell; pith of branchlets separating into thin plates ........ Juglans

I. Leaves with 5 to 9 leaflets; nut with dry, partly or wholly dehiscent husk and smooth bony shell; pith of branchlets continuous ............ Carya

Juglans nigra L.

$$
\text { Juglans }
$$

Java Farm and Ivy Neck. Infrequent to moderately abundant in mature canopies, usually associated with Liquidambar styraciflua in canopy and Lindera benzoin in understory. Dominant at Java Farm on steep east- and west-facing slopes of ridge near north boundary (hectare 2562); co-dominant with Quercus falcata, Fagus grandifolia, Platanus occidentalis, and Liquidambar styraciflua on south-facing slope above Muddy Creek (hectare 34I5, 34I6). Co-dominant at Ivy Neck with Quercus prinus at two points on shore of Scaffold Peninsula (hectares 5704, 5745). Higman I59, 755.

\section{Carya}

I. Terminal buds yellow, somewhat lanceolate. (Also, leaflets 5 to 9, lanceolate to lance-ovate $\ldots \ldots \ldots \ldots \ldots \ldots \ldots \ldots \ldots \ldots \ldots \ldots \ldots \ldots \ldots \ldots \ldots \ldots$ cordiformis

I. Terminal buds brown, ovoid-acute.

2. Leaflets 5 ; young branchlets glabrous ................ glabra

2. Leaflets 7 to 9; young branchlets tomentose ........... tomentosa

Carya cordiformis (Wangenh.) K. Koch

Bitternut Hickory (Carya cordiformis (Wang.) K. Koch)

Java Farm and Ivy Neck. Infrequent to moderately abundant in understory of mature forest, under canopy of Quercus alba, Fagus grandifolia, Quercus velutina, Liriodendron tulipifera, etc. Most abundant near top of south-facing slope overlooking tidal portion of Muddy Creek. Higman I70, 247, 403. 
Java Farm and Ivy Neck. Infrequent in understory of mature forest, like C. cordiformis above. Also observed under canopy of Quercus prinus. Associate species: Cornus florida, Acer rubrum, Carpinus caroliniana, Carya tomentosa, Liquidambar styraciflua. Higman 521.

C. tomentosa (Lam.) Nutt.

Mockernut Hickory

(C. tomentosa (Poir.) Nutt.)

Java Farm and Ivy Neck. Infrequent to moderately abundant in understory and canopy of mature forest. Associated with C. cordiformis and C. glabra above. Higman 4I8, 4I9, 420 .

\section{BETULACEAE}

I. Bark smooth, with rounded ridges (muscular); fruit a nutlet at the base of a lobed, leafy bract; nutlet wingless. ................. Carpinus

I. Bark smooth only on younger trees, without rounded ridges; fruit a number of small winged nutlets grouped into a cone, without leafy bracts.

2. Pith of branchlets triangular; cones woody, persistent, ovoid . . . . . A Alnus

2. Pith of branchlets circular; cones cylindrical .............. Betula

Carpinus caroliniana Walter

(Carpinus caroliniana Walt.)

\section{Carpinus}

Java Farm. Infrequent to moderately abundant in the understory of mature hardwood forest, under canopy of Fagus grandifolia, Quercus alba, Liriodendron tulipifera, etc. Seems to prefer slopes overlooking streams and freshwater marshes. Higman I87, II05.

\section{Alnus}

Alnus incana (L.) Moench ssp. rugosa (Du Roi) R.T. Clausen (Alnus rugosa (Du Roi) Spreng.) (A. serrulata (Air.) Willd., var. vulgaris)

Java Farm. One station: base of Fox Point peninsula, on north side. Hectare 3557. Canopy of Nyssa sylvatica, Quercus velutina, Quercus alba, Quercus prinus, Pinus virginiana. Associate species: Aralia spinosa, Prunus serotina, Acer rubrum. Higman 650. 
Java Farm and Ivy Neck. Infrequent along banks of estuaries, bottom of Muddy Creek valley and on Fox Point Road. Occasionally found under Quercus prinus canopy. Higman 651, iıo6.

\section{FAGACEAE}

I. Leaves simple, sharply and regularly serrate, acuminate; fruit a nut in a spiny bur. 2. Leaves oblong-ovate; leaf buds linear-lanceoloid and sharply pointed; bark pale gray; large tree . . . . . . . . . . . . . . . Fagus

2. Leaves oblong-lanceolate; leaf buds short and blunt; bark dark brown; stump

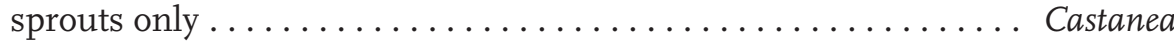

I. Leaves lobed, or if simple, entire or with round-tipped teeth; fruit a smooth

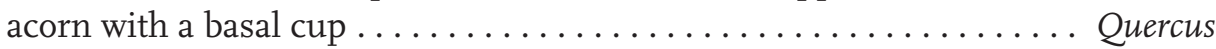

Fagus grandifolia Ehrh.

Fagus

American Beech

(Beech)

Java Farm. Moderately abundant in mature canopy, especially on upland west of Muddy Creek and on north- and west-facing slopes of ridge between old entrance road and main building area. Less frequent on lower south-facing slopes overlooking the tidal portion of Muddy Creek. Associate species: Quercus alba, Quercus velutina, Liriodendron tulipifera, Liquidambar styraciflua.

Ivy Neck. Infrequent in southern part of Quercus alba forest on Scaffold Peninsula. Higman 277, 558.

Castanea dentata (Marshall) Borkh.

Castanea

(Castanea dentata (Marsh.) Borkh.)

American Chestnut

(Chestnut)

Java Farm. Infrequent in mature mixed hardwood forest, occurring only as stump sprouts. Collected under canopy of Fagus grandifolia and its associates, also one station on Hog Island under canopy of Quercus prinus. Higman I07, 402.

\section{Quercus}

I. Lobes or teeth of leaves not bristle-tipped; acorn cup with puberulent scales, rust-puberulent within.

2. Leaves not lobed, or with sinuses less than I/3 of distance to midrib. (Also, leaves puberulent beneath; acorn puberulent above). 
3. Acorns on peduncles $2-7 \mathrm{~cm}$ long, exceeding petioles; leaves much paler and densely stellate-tomentose beneath, often shallowly lobed. Expected (Q. bicolor).

3. Acorns sessile or nearly so; leaves slightly paler and moderately stellatepuberulent beneath, coarsely serrate with sharp or rounded teeth (never lobed).

4. Leaves sharply serrate (tips of teeth round); acorn I.5-2.0 cm long, subglobose ........................... Q. muehlenbergii

4. Leaves dentate with broadly rounded teeth; acorn 2-3 cm long, ovoidellipsoid................................ prinus

2. Leaves lobed with sinuses more than $\mathrm{I} / 3$ of distance to midrib.

5. Mature leaves glabrous, whitened beneath, irregularly lobed (lobes ascending); acorn ovoid-ellipsoid, $2-3 \mathrm{~cm}$ long, glabrous ........... Q. alba

5. Mature leaves densely stellate-pubescent beneath, lobed like a cross; acorn ovoid, I-I. $5 \mathrm{~cm}$ long, puberulent above................. Q. stellata

I. Lobes of leaves (or tip in Q. phellos) bristle-tipped.

6. Leaves unlobed, entire. (Also, leaves glabrous beneath, acorn globose, IO-I5 $\mathrm{mm}$ long; acorn cup very shallow, with appressed scales, nearly sessile) ................................... Q phellos

6 . Leaves lobed, the lobes usually cleft.

7. Leaves broadly obovate, shallowly 3-lobed. (Also, leaves rounded at base, minutely rust-puberulent beneath; acorn cup turbinate, with tomentose scales, covering about $\mathrm{I} / 2$ of nut) $\ldots \ldots \ldots \ldots \ldots \ldots$ Q. marilandica

7. Leaves oblong or elliptic in general outline, moderately to deeply 5- to 7-lobed.

8. Base of leaf blade rounded to petiole, often unevenly; basal lobes broadly divergent, terminal one long and narrow. (Also, acorn cup deeply saucershaped, covering $\mathrm{I} / 3$ of nut, its scales appressed, rounded) .... Q. falcata

8. Base of leaf blade tapering to petiole; lobes all ascending-spreading. (Also, leaves often pubescent only in axils of veins beneath).

9. Acorn cup turbinate, covering more than $\mathrm{I} / 3$ of nut; leaf buds tomentose.

Io. Upper scales of acorn cup loosely imbricated, forming a fringe, pubescent .......................... Q velutina

Io. Upper scales of acorn cup appressed, becoming glabrous. (Also, leaf sinuses often broadly circular; acorns often with pale concentric rings around the tip) $\ldots \ldots \ldots \ldots \ldots \ldots$. coccinea

9. Acorn cup saucer-shaped, covering about I/4 of nut; leaf buds usually glabrous (or tomentose above in Q. rubra).

II. Leaves lobed moderately, bottom of sinus rarely more than $2 / 3$ the distance from lobe-tip to midrib; acorn cup I.5-3 cm wide; nut 2-3 cm long. (Also, bark often with pale gray streaks on upper trunk; forest habitat). Expected (Q. rubra). 

tance from lobe-tip to midrib; acorn cup I-I. $6 \mathrm{~cm}$ wide; nut I-I.3 $\mathrm{cm}$ long. (Also, lower branches often spreading downward; frequently in old field habitat) $\ldots \ldots \ldots \ldots \ldots$. palustris

Quercus muehlenbergii Engelm.

Chinquapin Oak (Quercus muehlenbergii Engelm.?)

(Q. prinoides Willd. var. acuminata (Michx) Gl.)

(Yellow Oak)

Java Farm. Infrequent, large trees overhanging Rhode River (Fox Creek estuary). Adjacent forest canopy of Robinia pseudoacacia and Ulmus americana dominants. Higman 766.

Q. montana Willd.

Chestnut Oak

(Q. prinus L.)

Java Farm. Co-dominant with Quercus alba in canopy at Fox Point and Hog Island. Hectares 3558 and 3592. Associate canopy species include Carya glabra and Carya tomentosa. Understory dominants are Pinus virginiana (at Fox Point under thin canopy) and Kalmia latifolia (at Hog Island). Understory associates include Ilex opaca, Acer rubrum, and Liquidambar styraciflua. Ericaceous shrubs numerous. Also one station in bottom of Muddy Creek valley, with canopy dominant Fagus grandifolia. Hectare 3403 .

Ivy Neck. Dominates most of the shoreline of lower Scaffold Peninsula, usually on banks 6 to Io feet above the estuary. Co-dominants include Quercus alba, Carya tomentosa, Acer rubrum, and locally Fagus grandifolia. On Cheston Peninsula (west shore) Quercus velutina usually co-dominates. Understory resembles that at Java Farm. Higman 26I.

Q. alba L.

White Oak

Java Farm and Ivy Neck. Moderately abundant in most hardwood canopies, on dry to moderately moist sites. Co-dominant on upland with Quercus velutina, Acer rubrum, Liriodendron tulipifera, Carya Tomentosa, Fagus grandifolia, etc. Often codominant with Quercus prinus on estuary shores. Higman 263.

Q. stellata Wangenh.

Post Oak

(Q. stellata Wang.)

Java Farm. Infrequent on banks of tidal portion of Muddy Creek, under canopy of Quercus velutina and Quercus alba. One station on Fox Point, under canopy of Quercus prinus. Prefers the shoreline, overhanging the water. Also adventive in Hog Island salt marsh. Higman 260, 275, 460. 
Java Farm. One station in Hog Island salt marsh. Hectare 3572. Associate species: Pinus virginiana, Juniperus virginiana, Liquidambar styraciflua, Quercus stellata, Quercus palustris, Myrica cerifera. Higman 278, 591.

Q. marilandica Münchh.

Blackjack Oak

(Q. marilandica (L.) Muench.)

(Black-jack Oak)

Java Farm and Ivy Neck. Infrequent, associated with Pinus virginiana. Also at Java Farm: one station west of Old Muddy Creek Road, in canopy of Liriodendron tulipifera and Fagus grandifolia. Higman 407.

Q. falcata Michx. (L)

Southern Red Oak

(Spanish Oak, Red oak)

Java Farm. Moderately abundant in canopy of bottomland forest at northwest corner of Hog Island salt marsh. Hectares 356r, 357I. Adventive into the marsh. Infrequent on south-facing slope along tidal portion of Muddy Creek, with dominants Quercus alba, Fagus grandifolia, and Quercus velutina. Also one station on Fox Point. Hectare 3558. Canopy there of Quercus prinus.

Ivy Neck. One station on east bank of Scaffold Peninsula. Canopy of Quercus alba, Quercus velutina. Higman 9I, 262, 404.

Q. velutina Lam.

Black Oak

Java Farm and Ivy Neck. Moderately abundant in most mature hardwood canopies, especially on upper slopes and moderately dry sites. Also moderately abundant on estuary shore of Cheston Point, where Quercus prinus would be expected. Higman 588.

Q. coccinea Münchh.

Scarlet Oak

(Q. coccinea Muench.)

Java Farm. Infrequent in canopy of mature upland forest west of Muddy Creek; also on ridge west of main building area and on south-facing slope overlooking meadow, north of main buildings. Canopy dominants include Fagus grandifolia, Quercus alba, Liquidambar styraciflua, and Liriodendron tulipifera. Higman 4I4, 557.

Q. palustris Münchh.

Pin Oak

(Q. palustris Muench.)

Java Farm and Ivy Neck. Moderately abundant adventive in abandoned fields; associate species: Acer rubrum, Liquidambar styraciflua, Cornus florida, etc. Also at Java Farm: infrequent in valley of Muddy Creek, under canopy of Platanus occidentalis and Fraxinus americana. Higman 75, I69. 


\section{ULMACEAE}

I. Leaves entire, glabrous, prominently 3-veined at base, very oblique at base, usually with insect galls; buds appressed to stem; fruit a dark green drupe . . . . . . Celtis

I. Leaves serrate or dentate, pubescent or roughened on both surfaces (except U. pumila), moderately to slightly oblique at base, without insect galls; buds somewhat spreading; fruit a samara $\ldots \ldots \ldots \ldots \ldots \ldots$ Ulmus

Celtis occidentalis L.

\section{Celtis}

Common Hackberry

(Hackberry, Sugarberry)

Java Farm. Moderately abundant in old fields and along roadsides, especially those with oyster shell paving. Prefers calcareous soil.

Ivy Neck. One station at old house, between entrance road and source of Scaffold Creek drainage. Hectare 4658. Higman 367, 370, 463, 473, 483, 633, 773.

\section{Ulmus}

I. Leaves smooth, simply serrate, very slightly oblique at base; samaras entirely

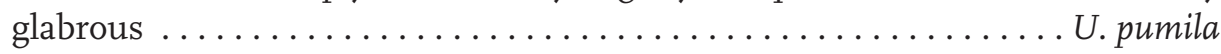

I. Leaves rough or harsh above, doubly serrate, definitely oblique at base; samaras pubescent.

2. Leaves slightly folded at the midrib, very harsh above, ciliate; buds and branchlets downy pubescent; samaras pubescent only in the center ....... U. rubra

2. Leaves flat, moderately scabrous above, glabrous; buds and branchlets glabrous or sparingly pilose; samaras pubescent only on the margins ....U. americana

\section{Ulmus pumila L.}

Siberian Elm

(Ulmus pumila L.)

(Dwarf Elm)

(not in Britton \& Brown)

Ivy Neck. One station: edge of bank above Rhode River, just north of Sand Point. Hectare 4769. Associate species: Maclura pomifera, Liquidambar styraciflua. Higman I074.

U. rubra Muhl.

Slippery Elm

Java Farm. Infrequent near northeast corner and in vicinity of pier. Associate species: Fraxinus tomentosa, Ulmus americana, Celtis occidentalis. Higman 347, 39I, 6i9, 77i. 
Java Farm. Infrequent in old fields north of main building area. Associate species: Celtis occidentalis, Liquidambar styraciflua, Quercus palustris. One station along shore of Muddy Creek estuary east of Hog Island salt marsh. Associate species there: Robinia pseudoacacia, Cornus florida, Liquidambar styraciflua, Prunus avium, Morus rubra.

Ivy Neck. Infrequent in Quercus alba forest of central Scaffold Peninsula. Higman 428 .

\section{MORACEAE}

I. Leaves entire; branches with axillary spines; staminate flowers in rounded racemes; mature syncarp (fleshy aggregate fruit) 7 to $15 \mathrm{~cm}$ in diameter, with a

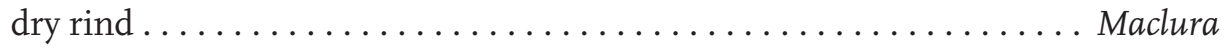

I. Leaves coarsely serrate, often lobed; branches spineless; staminate flowers in long aments; mature syncarp I to $2 \mathrm{~cm}$ thick.

2. Buds with 2 or 3 scales; syncarp globose, with protruding fruits . . . . . . .

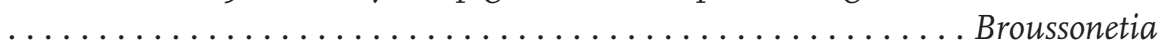

2. Buds with 3 to 6 scales; syncarp cylindric or ellipsoid, juicy, the fruits not

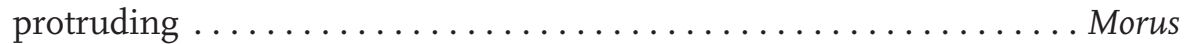

\section{Maclura}

\section{Maclura pomifera (Raf.) C.K. Schneid.}

(Maclura pomifera (Raf.) Schneid.)

Osage Orange

Ivy Neck. Infrequent to moderately abundant along coast of upper Cheston Peninsula, both on the Cheston Creek and Rhode River sides. Also in hedgerows and along drainage of Cheston Creek above the estuary. Higman 869, ıого.

Broussonetia papyrifera (L.) L'Hér. ex Vent.

\section{Broussonetia}

(Broussonetia papyrifera (L.) Vent.)

Java Farm. One station: west fence of field at northeast corner of Farm. Hectare 3600. Associate species: Liquidambar styraciflua, Ulmus Americana, Ulmus rubra. Higman 346, II37.

\section{Morus}

I. Leaves densely pubescent on veins and surface beneath .......... rubra

I. Leaves pubescent only in axils of veins beneath ............... alba 
Java Farm and Ivy Neck. Infrequent in understory of a variety of mature hardwood canopies; seems to prefer low stream banks, poorly drained woods, etc. Higman $425,527,770$.

M. alba L.

White Mulberry

Java Farm and Ivy Neck. Infrequent, same habitat as Morus rubra above. Higman 479 .

\section{URTICACEAE}

Boehmeria cylindrica (L.) Sw.

Smallspike False Nettle

(False Nettle)

Java Farm. Infrequent along old entrance road near culvert of Muddy Creek and along new entrance road near culvert of Fox Creek drainage. Both sites densely shaded by adjacent forest. Associate species: Lycopus virginicus, Cryptotaenia canadensis, Agrimonia microcarpa, Elephantopus carolinianus. Higman 458, 978.

\section{POLYGONACEAE}

I. Outer sepals wide-spreading or reflexed, inner ones (valves) enlarged in fruit ... . . . . . . . . . . . . . . . . . . . . . . . . . . . . . . . . . Rumex

I. All sepals equal, erect or ascending and petaloid.

2. Styles 2, deflexed and hooked, persistent on achene as rigid beaks; flowers remote on terminal raceme, deflexed in fruit . . . . . . . . . . Tovara

2. Styles 2-3, not deflexed or hooked, deciduous; flowers in dense raceme, not

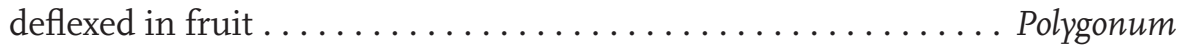

\section{Rumex}

I. Leaves hastate, with 2 spreading basal lobes; racemes red or yellowish; flowers dioecious; plant spreading by slender horizontal rootstocks $\ldots . . R$. acetosella

I. Leaves without basal lobes, entire or with crisped or uneven margins; racemes greenish, brown in fruit; flowers monoecious or perfect; vertical taproot.

2. Margins of leaves entire, not crisped or uneven. (Also, margins of valves entire).

3. Salt marsh or sub-aquatic habitat; leaves narrowly elliptic; fruiting pedicels clavate and reflexed ...................... verticillatus

3. Terrestrial habitat; leaves oblong-lanceolate, with cordate bases in one species; fruiting pedicels slender, not reflexed. Expected (R. altissimus). 
2. Margins of leaves undulate or crisped.

4. Valves entire or obscurely dentate. (Also, valves broadly ovate; leaves with

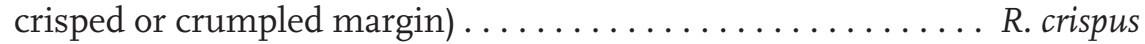

4. Valves with long teeth. (Also, habitat not saline; margins of leaves slightly uneven).

5. Plant $2-7 \mathrm{dm}$ high; length of panicle more than $\mathrm{I} / 2$ height of plant . .

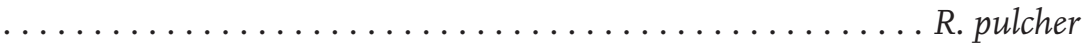

5. Plant 6-I5 dm high; length of panicle less than $\mathrm{I} / 2$ height of plant ... $\ldots \ldots \ldots \ldots \ldots \ldots \ldots \ldots \ldots \ldots \ldots \ldots \ldots \ldots \ldots \ldots \ldots \ldots \ldots$ obtufolius

Rumex acetosella L.

Common Sheep Sorrel

(Red Sorrel)

Java Farm and Ivy Neck. Infrequent to moderately abundant in dry, open soil (abandoned fields, roadsides, etc.) Higman 590, 759, I083.

R. verticillatus L.

Swamp Dock

(Water Dock)

Java Farm. Moderately abundant in salt marshes along Muddy Creek. Associated with Panicum virgatum, Scirpus spp.

Ivy Neck. Moderately abundant in estuarine salt marshes and at coastal overflow ponds on Cheston Peninsula. Higman 289, 945.

R. crispus L.

Curly Dock

(Sour Dock)

Ivy Neck. Infrequent in abandoned wheatfield. Also one station in dry, sandy halophytic marsh on tip of Scaffold Peninsula. Higman 688, 852, 915, 975.

R. pulcher L.

Fiddle Dock

(Dock)

Java Farm. One station: grassy patch at entrance gate. Hectare 2463. Associated with Cyperus strigosus, Perilla frutescens, Trifolium spp. Higman io84.

R. obtusifolius L.

Bitter Dock

Java Farm. One station: on Fox Point Road just before turn eastward to Fox Point; in front of old barn. Hectare 3543.

Ivy Neck. One station: abandoned road through forest on Scaffold Peninsula. Canopy of Liquidambar styraciflua, Robinia pseudoacacia, Nyssa sylvatica, Morus rubra. Higman 523, 976. 


\section{Polygonum}

(Tovara)

Polygonum virginianum L.

Jumpseed

(Tovara virginiana (L.) Adans.)

(Polygonum virginianum L.)

(Virginia Knotweed)

Java Farm. Infrequent; seems to prefer moist, partly shaded sites. At entrance gate; along entrance road under canopy of Quercus alba, Fagus grandifolia, Liquidambar styraciflua, etc.; on moist valley of Muddy Creek under canopy of Fraxinus americana and Platanus occidentalis; and at main buildings. Higman 38, 65, 354, 538.

\section{Polygonum}

I. Plant prostrate-spreading; flowers in axillary fascicles . . . . . . . P. aviculare

I. Plant erect; flowers in spiciform racemes or panicles, or capitate.

2. Stem prickly; leaves sagittate or hastate.

3. Leaves sagittate; inflorescence capitate ........... P. sagittatum

3. Leaves hastate, the basal lobes horizontally spreading; inflorescence a short raceme or panicle .................. arifolium

2. Stem smooth; leaves elliptic.

4. Ocreae (sheathing stipules) without marginal cilia.

5. Leaves strigose; peduncles and axis with stalked glands.......... $\ldots \ldots \ldots \ldots \ldots \ldots \ldots \ldots \ldots \ldots \ldots \ldots \ldots \ldots \ldots \ldots$ p. pensylvanicum

5. Leaves glabrous to scabrous; peduncles and axis glandless. (Also, ocreae short-strigose; base of plant creeping) . . . . . . . P. hydropiperoides

4. Ocreae with marginal cilia (lower ocreae may have top torn off).

6. Plant annual, with fibrous roots.

7. Mature calyx glandular-punctate.

8. Calyx greenish; achene dull. Expected (P. hydropiper).

8. Calyx white; achene lustrous . ............ Punctatum

7. Mature calyx glandless.

9. Leaves ciliate on margins and lower veins; spikes terminal, 3-5 mm thick; cilia of ocreae equal to or longer than the sheath ... P. cespitosum, var. longisetum

9. Leaves glabrous, or sparsely strigose beneath; spikes in panicles, 7-II mm thick; cilia of ocreae shorter than the sheath. (Also, leaves often with purple blotch above but this character not consistent) $\ldots \ldots \ldots \ldots \ldots \ldots \ldots \ldots \ldots \ldots \ldots \ldots \ldots \ldots \ldots \ldots \ldots \ldots \ldots$

6. Plant perennial, with subligneous forking rootstocks.

Io. Mature calyx glandular-punctate ........... punctatum

Io. Mature calyx glandless.

II. Calyx pink; leaves glabrous to scabrous; ocreae short-strigose .P. hydropiperoides 
II. Calyx white; leaves strigose; ocreae strigose-hirsute

$$
\text { P......... }
$$

See also Appendix I, p. 227: P. cristatum.

Polygonum aviculare L.

Prostrate Knotweed

(Smartweed)

Ivy Neck. One station: unpaved road to abandoned wheatfield. Hectare 4740. Associated with Diodia teres, Lespedeza stipulacea, Polygonum pensylvanicum, Cyperus strigosus. Higman I059.

P. sagittatum L.

Arrowleaf Tearthumb

(Smartweed)

Java Farm. Two stations: freshwater part of Fox Creek marsh (associated with Aster puniceus, Lycopus virginicus, Leersia oryzoides, Solidago graminifolia, Impatiens capensis), and in marshy western end of Phalaris arundinacea meadow north of main buildings (associated with Polygonum punctatum, P. pensylvanicum, Scirpus rubricosus). Higman I52.

P. arifolium L.

Halberdleaf Tearthumb

(Smartweed)

Java Farm. One station: low bank of Muddy Creek, adjacent to west end of meadow above. Hectare 2467. Canopy of Platanus occidentalis, Fagus grandifolia, Liriodendron tulipifera. Understory of Lindera benzoin, Cornus florida. Higman I055.

P. pensylvanicum L.

Pennsylvania Smartweed

(Smartweed)

Java Farm. One station: large clump at marshy western end of Phalaris arundinacea meadow north of main buildings. Hectare 2467. Associates: see P. sagittatum above.

Ivy Neck. Infrequent in open fields, unpaved roads. Associated with Strophostyles umbellata, Desmodium perplexum, Lespedeza stipulacea, Oenothera biennis. Higman I5I, IO23, II23, II24.

P. hydropiperoides Michx.

Swamp Smartweed

(P. hydropiperoides Michx., forma leucochranthum A. H. Moore) ～(Smartweed) (form not in Britton and Brown)

Java Farm. One station: border of salt marsh at upstream end of tidal portion of Muddy Creek. Hectare 3403. Associated with Mikania scandens, Pluchea camphorate, Lobelia cardinalis, Hibiscus palustris, Typha latifolia. Higman 80. 
Java Farm. Infrequent in fresh and brackish marshes. Stations in Hog Island salt marsh, muddy shore of tidal portion of Muddy Creek, in Fox Point salt marsh, and at marshy west end of Phalaris arundinacea meadow north of main buildings.

Associates in freshwater marsh: see P. sagittatum above.

Associates in salt marshes: Aster puniceus, Atriplex patula, Scirpus americanus, Distichlis spicata, Kosteletzkya virginica. Higman 58, I53, 449, 990, I245.

$P$. punctatum Elliott var. confertiflorum (Meisn.) Fassett

Dotted Smartweed

(P. punctatum, var. Leptostachyum (Meisn.) Small)

(Smartweed)

(variety not in Britton and Brown)

Java Farm. One station: large abandoned field south of entrance road and east of road to springhouse. Solidago graminifolia, Rubus spp., Rhus radicans, and Lonicera japonica dominant. Higman I32.

P. cespitosum Blume var. longisetum (Bruijn) A.N. Steward Oriental Lady's Thumb ( $P$. cespitosum Blume, var. longisetum (deBruyn) Stewart)

(Smartweed)

Java Farm. Three stations: along entrance road between gate and Muddy Creek (canopy of Quercus alba, Fagus grandifolia, Liquidambar styraciflua, etc.; associate species Desmodium spp., Agrimonia microcarpa, Elephantopus caroliniana, Tovara virginiana); two stations along Fox Creek ditch (canopy of Juglans nigra, Liquidambar styraciflua; associate species Perilla frutescens, Duchesnea indica, Eupatorium serotinum, Geum canadense).

Also observed along stream at northeast border of farm. Higman 35, 223, 3I4, 922.

P. persicaria L.

Spotted Ladysthumb

(Smartweed)

Ivy Neck. One station: near small barn on south bank of artificial pond, Cheston Peninsula. Hectare 4723. Dry, unshaded. Associate species: Potentilla norvegica, Ipomoea hederacea, Abutilon theophrasti, Eleusine indica. Higman Ioo2.

P. setaceum Baldw.

Bog Smartweed

(P. setaceum Baldw. (P. hydropiperoides, var. setaceum (Baldw.) Gl.)) (Smartweed)

Java Farm. One station: north boundary fence, adjacent neighbor's cornfield. Associate species: Ambrosia trifida, Clematis virginiana, Amaranthus hybridus. Higman I243. 


\section{CHENOPODIACEAE}

I. Leaves alternate, or the lower opposite in Atriplex, flat, neither reduced to scales nor spine-tipped. (Also, plants erect; stems and branches green with white longitudinal stripes; flowers in small, spicate clusters).

2. Terrestrial habitat; flowers perfect; calyx 5-parted. (Also, leaves narrowly to broadly lanceolate, entire to dentate) $\ldots \ldots \ldots \ldots \ldots \ldots$ Chenopodium

2. Salt marsh habitat; flowers unisexual, monoecious or dioecious; calyx absent in pistillate flowers, these enclosed by two appressed bracts. (Also, in species collected, leaves hastate; spikes reddish) .................. Atriplex

I. Leaves opposite and reduced to scales, or alternate and spine-tipped. Expected (Salicornia, Salsola).

\section{Chenopodium}

I. Leaves and inflorescence glandular, aromatic. Expected (C. ambrosioides, C. botrys).

I. Leaves and inflorescence not glandular but often farinose (covered with mealy substance in dry specimens).

2. Principal leaves deltoid or rhomic too broadly ovate, their margins serrate or sinuate. (Also, seeds all horizontal if collected in late autumn).

3. Leaves with lustrous upper surface, coarsely serrate margin, and long petiole, usually not farinose; inflorescence short, spreading, branched. Expected (C. murale).

3. Leaves with dull or glaucous upper surface, sinuate-dentate margin, tapering to short petiole, slightly too densely farinose; inflorescence of dense clusters, these forming long, short-branching, continuous or interrupted spikes. (Spikes may be shorter than leaves in C. rubrum).

4. Terrestrial habitat.

5. Leaves glaucous above, densely farinose beneath; young stem greenish; fresh calyx-lobes barely keeled. (Also, leaves and inflorescence often red in late autumn; leaves subtending the individual spikes narrowly linear, unlike the serrate cauline leaves) ........ C. album

5. Leaves dark green or yellowish, slightly farinose beneath; young stem red or purple; fresh calyx-lobes strongly keeled. Expected (C. paganum).

4. Salt marsh habitat. (Also leaves and inflorescence red at maturity. Seeds often vertical) $\ldots \ldots \ldots \ldots \ldots \ldots \ldots \ldots \ldots \ldots$. . rubrum

2. Principal leaves lanceolate, elliptic, narrowly ovate or oblong, the margins usually entire (often sinuate in C. glaucum). Expected (C. glaucum, C. leptophyllum, C. lanceolatum). 
Java Farm. One station: entrance road near road to springhouse. Hectare 3408. Dry, unshaded site.

Ivy Neck. One station: abandoned wheatfield. Hectare 4772. Higman 982, II2I.

C. rubrum L.

Red Goosefoot

(Goosefoot)

Java Farm. Probably in salt marsh near Fox Point Peninsula. (Collected by H. David Hammond).

Atriplex prostrata Bouchér ex DC.

Atriplex

(Atriplex patula L., var. hastata (L.) Gray)
Triangle Orache

(Spearscale)

Java Farm and Ivy Neck. Moderately abundant in salt marshes. Associate species: Polygonum punctatum, Distichlis spicata, Scirpus robustus, Kosteletzkya virginica, Rumex verticillatus, Pluchea camphorata, etc. Higman 60, 276, 282, 450.

\section{AMARANTHACEAE}

I. Leaves alternate, anthers 2-locular. (Also, flowers unisexual; filaments separate).

2. Salt marsh habitat; flowers dioecious; leaves lance-linear; spikes remotely flowered in loose, spreading panicle . . . . . . . . . . . . Acnida

2. Terrestrial habitat; flowers monoecious; leaves lance-ovate; spikes densely flowered in compact, erect panicle.............. Amaranthus

I. Leaves opposite; anthers I-locular. Expected (Iresine).

\section{Amaranthus}

(Acnida)

\section{Amaranthus cannabinus (L.) Sauer}

(Acnida cannabina L.)
Tidalmarsh Amaranth

(Water Hemp)

Java Farm and Ivy Neck. Infrequent in salt marshes, seems to prefer drier parts of marsh, also sandbars or low hummocks. Associate species: Distichlis spicata, Spartina patens, Panicum virgatum, Baccharis halimifolia, Gerardia maritima, Juniperus virginiana. Higman 99, 971.

\section{Amaranthus}

I. Spines absent from axils of leaves; utricle circumscissle, the top falling off as a lid.

2. Plant slender; blades of principal leaves 0.5 to $7 \mathrm{~cm}$ long; flowers in small axillary clusters; stamens 2 or 3 . Expected (A. albus). 
2. Plant stout; blades of principal leaves 5 to $30 \mathrm{~cm}$ long; flowers monoecious in terminal and axillary simple or panicled spikes; stamens 5 .

3. Calyx of pistillate flowers about $3 \mathrm{~mm}$ long, the bracts 2 to 3 times this length. Expected (A. retroflexus).

3. Calyx of pistillate flowers I.5 to $2 \mathrm{~mm}$ long, the bracts slightly longer to twice as long.

4. Lateral spikes of terminal panicle divergent; bracts red or purple; utricle longer than calyx. Expected (A. cruentus).

4. Lateral spikes of terminal panicle ascending to erect; bracts green to red-tinged; utricle shorter than to equaling the calyx ...... A. hybridus

I. Spines paired in axils of leaves; utricle bursting irregularly . . . . . A. spinosus

Amaranthus hybridus L.

Slim Amaranth

(Prince's Feather, Amaranth)

Java Farm. One station: north boundary fence, adjacent to neighbor's cornfield. Associate species: Clematis virginiana, Ambrosia trifida.

Ivy Neck. One station: rubbish pile between old house and tobacco barn, near road. Hectare 4658. Associate species: Juncus tenuis, Barbarea vulgaris, etc. Higman I037, III7.

A. spinosus L.

Spiny amaranth

(Thorny Amaranth)

Java Farm. One station: rubbish pile near old house, at main buildings. Hectare 2580. Associate species: Solanum nigrum, Eleusine indica, Datura stramonium, Juncus tenuis, Melilotus alba. Higman 500.

\section{PHYTOLACCACEAE}

Phytolacca

Phytolacca americana L.

American Pokeweed

(Pokeweed, Pokeberry)

Java Farm. Infrequent around main buildings and in abandoned fields.

Ivy Neck. Apparently an initial dominant in abandoned fields on Scaffold Peninsula. Moderately abundant in stands of young Liquidambar styraciflua and Acer rubrum. Infrequent in hardwood forests of Scaffold and Cheston Peninsulas, under canopy of Liquidambar styraciflua, Juglans nigra, Robinia pseudoacacia, Nyssa sylvatica, etc. Higman 3. 


\section{MOLLUGINACEAE \\ (AIZOACEAE) \\ Mollugo}

Mollugo verticillata L.

Green Carpetweed

(Carpet-weed)

Ivy Neck. Infrequent in cornfields. Associate species: Ipomoea hederacea, Barbarea verna, Draba verna, Lepidium virginicum, etc. Higman 958, ro62.

Claytonia virginica $\mathrm{L}$.

Virginia Springbeauty

(Spring Beauty)

Java Farm. Moderately abundant in mature forest in vicinity of old entrance road and gate. Canopy of Acer rubrum, Quercus alba, Liquidambar styraciflua, etc. Associate species: Sanguinaria canadensis, Dentaria laciniata, Desmodium spp. Higman 562.

\section{CARYOPHYLLACEAE}

I. Sepals distinct; petals, if present, without basal claws.

2. Leaves with scarious stipules. Expected (Spergularia).

2. Leaves without stipules.

3. Petals not apically notched or deeply 2-cleft. (Petals may be absent in Sagina). Expected (Sagina, Arenaria, Holosteum).

3. Petals apically notched or 2-cleft.

4. Styles 3; capsule ovoid or ellipsoid; flowers solitary or in cymes ......

Stellaria

4. Styles 5; capsule cylindric; flowers not in umbels but may be clustered

I. Sepals united into a tube; petals, if present, with basal claws.

5. Petals absent. (Also, calyx a hard cup; flowers green, sessile in upper axils). Expected (Scleranthus).

5. Petals present.

6. Petals red-violet; calyx with scarious or leafy basal bracts....... Dianthus

6. Petals white or pinkish; calyx without basal bracts.

7. Styles 5; capsule opening by 5 or to teeth $\ldots \ldots \ldots \ldots \ldots$. Lychnis

7. Styles 2 or 3; capsule with 3, 4, or 6 valves.

8. Calyx ı-nerved; styles 3; capsule 3- or 6-valved .......... Silene

8. Calyx obscurely nerved and cylindric or 5-nerved and ovoid; styles 2; capsule 4-valved. Expected (Saponaria). 
Java Farm. Infrequent to abundant along roadsides and in sparsely shaded old fields. Principal station near junction of old entrance road and base line. Canopy of Platanus occidentalis and Fraxinus americana. Associate species: Ranunculus sceleratus, Duchesnea indica, Lonicera japonica.

Ivy Neck. Infrequent in cornfields and abandoned grassy field. Higman 550, 753.

\section{Cerastium}

I. Plants perennial, with long, matted, creeping basal branches. (Also, bracts of inflorescence scarious-margined or the lowest ones herbaceous).

2. Basal leafy branches herbaceous, without axillary tufts, their leaves hirsute

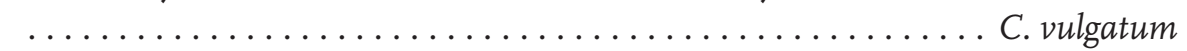

2. Basal leafy branches becoming dry and withering but persistent, with conspicuous axillary tufts, their leaves not hirsute. Expected (C. arvense var. villosum).

I. Plants annual or winter-annual, without creeping basal branches.

3. Leaves narrowly oblong to oblanceolate; capsules 2-3 times as long as sepals; sepals oblong, blunt or acute. Expected (C. nutans).

3. Leaves broadly elliptic to ovate or obovate; capsules I-2 times as long as sepals; sepals lance-attenuate.

4. Bracts of inflorescence scarious-margined. Expected (C. semidecandrum). 4. Bracts of inflorescence herbaceous. (Also, sepals scarious-margined).... C. viscosum

Cerastium fontanum Baumg. ssp. vulgare (Hartm.)

\section{Greuter \& Burdet}

(Cerastium vulgatum L.)

Big Chickweed

(Mouse-ear Chickweed)

Ivy Neck. Abundant in fallow cornfields. Associate species: Cerastium viscosum, Stellaria media, Draba verna, Barbarea vulgaris, etc. Also one station on dike through salt marsh at head of Cheston Creek estuary. Higman 868.

C. glomeratum Thuill.

(C. viscosum L.)

Sticky Chickweed (Mouse-ear Chickweed)

Java Farm. Infrequent in grassy old fields between south side of main building area and old entrance road. Associate species: Poa pratensis, Cirsium vulgare, Asclepias syriaca.

Ivy Neck. Moderately abundant in fallow cornfields. Associated with C. vulgatum above. Higman 636 . 


\section{Dianthus}

Dianthus armeria L.

Deptford Pink

Java Farm. Infrequent along unshaded portions of Fox Point Road and new entrance road, and in old field south of Fox Creek marsh.

Ivy Neck. Infrequent along tractor trails and in abandoned grassy field. Higman 23,722 .

\section{Silene \\ (Lychnis)}

Silene latifolia Poir. ssp. alba (Mill.) Greuter \& Burdet

Bladder Campion

(Lychnis alba Mill.)

(White Campion)

Java Farm. Infrequent to moderately abundant in grassy old fields south of main building area. Associate species: Cerastium viscosum above. Higman 8, 662.

I. Calyx glabrous or virtually so.

Silene

2. Stem not sticky; calyx somewhat inflated, its nerves not prominent.

3. Leaves in whorls of 4 ; petals fringed .............. stellata

3. Leaves opposite; petals 2-lobed. Expected (S. cucubalus, S. nivea).

2. Stem with sticky areas; calyx tight around the capsule, its 5 to Io straight nerves prominent. (Also, flowers in loose panicles, with long pedicels; petals

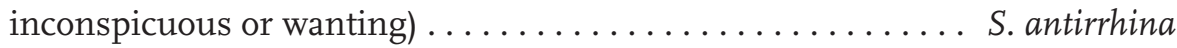

I. Calyx pilose. (Also, plants annual, without persistent bases; calyx prominently 5- to Io-nerved; inflorescence an open cymose panicle) . . . . . . . S. noctoflora

Silene stellata (L.) W.T. Aiton

Widowsfrill

(Silene stellata (L.) Ait.)

(Starry Campion)

Java Farm. One station: large mound on bottom of Muddy Creek valley, between old entrance road and Muddy Creek. Hectare 2497. Canopy of Quercus alba, Quercus velutina, Fagus grandifolia, Carya glabra. Higman io4I.

S. antirrhina L.

Sleepy Silene

(Catchfly)

Ivy Neck. One station: southern part of grassy abandoned field on Scaffold Peninsula. Hectare 4772. Higman 693.

S. noctiflora L.

(S. noctoflora L.)
Nightflowering Silene

(Catchfly, Campion)

Java Farm. One station on Fox Point Road. Higman 803. 


\section{CABOMBACEAE}

(NYMPHACEAE)

Brasenia

Brasenia schreberi J.F. Gmel.

Watershield

(Brasenia schreberi Gmel.)

(Water-shield)

Java Farm. One station on shore of tidal portion of Muddy Creek, near mouth of small tributary below springhouse. Hectare 3437. Growing on soft mud. Associate species: Panicum virgatum, Pluchea Camphorate, Polygonum punctatum. Higman 998.

\section{RANUNCULACEAE}

I. Pistils forming follicles (pods opening down one side) or berries. Expected (Caltha, Coptis, Aquilegia, Delphinium, Aconitum, Cimicifuga, Actaea).

I. Pistils forming achenes or utricles borne in heads or dense spikes.

2. Plant a vine; leaves opposite; sepals 4 , petaloid (petals absent); styles long and

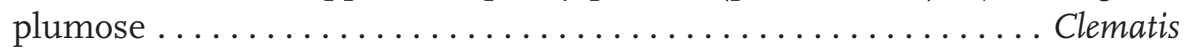

2. Plant erect; leaves alternate or radical, the upper sometimes opposite or whorled; sepals 3 to 20 .

3. Petals 5 , yellow; sepals $5 \ldots \ldots \ldots \ldots \ldots \ldots$ Ranunculus

3. Petals absent or represented by modified stamens; sepals 3 to 20 .

4. Leaves all alternate; flowers in racemes, panicles, or corymbs. (Also, leaves 2-to 3 -temately compound; flowers dioecious) ...... Thalictrum

4. Leaves at summit of stem or peduncle opposite or whorled, forming an involucre; flowers solitary or in umbels.

5. Basal leaf I, involucral leaves compound (3 leaflets); roots tuberous; sepals 5 to Io, petaloid. (Also, sepals white or pink) ..... Anemonella

5. Basal leaves several, basal and involucral leaves simple roots not tuberous; sepals 4 to 6 .

6. Involucral leaves unlobed, near the flower, sepals bluish, white or pink; plant stemless. Expected (Hepatica).

6. Involucral as well as basal leaves with 3 to 5 deeply lobed divisions; involucral leaves remote from the flower; sepals greenish-yellow; plant with stem ........................ Anemone

\section{Clematis}

I. Leaflets toothed or dissected; sepals 6 to I2 $\mathrm{mm}$ long; achenes pilose or villous-

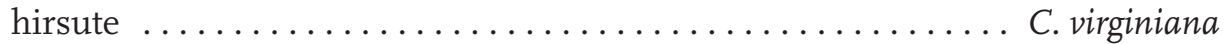

I. Leaflets entire or wavy-margined; sepals Io to I7 $\mathrm{mm}$ long; achenes appressedsilky .................................. dioscoreifolia 
Java Farm. Infrequent in old fields, among Rhus radicans and Lonicera japonica. Higman I30, 710, I038.

C. terniflora DC. var. terniflora
(C. dioscoreifolia Levi \& Vaniot)

\author{
Sweet Autumn Virginsbower
}

(Clematis)

Java Farm. One station: steep southeast-facing slope at shore of Rhode River, slightly below pier. Hectare 36ro. Shaded by Robinia pseudoacacia. Associate species Lonicera japonica. Higman I039.

\title{
Ranunculus
}

I. Basal leaves simple or lobed, cauline ones divided; roots fibrous, without corm.

2. Habitat aquatic or amphibious; plant fleshy; the lower and middle cauline leaves long-petioled ...................... sceleratus

2. Habitat terrestrial; plant not fleshy; cauline leaves short-petioled to sessile. (Also, achene beakless; basal leaves shallowly dentate or crenate) . . . . . . . $\ldots \ldots \ldots \ldots \ldots \ldots \ldots \ldots \ldots \ldots \ldots \ldots \ldots \ldots \ldots \ldots \ldots$ abortivus

I. Basal and cauline leaves compound; roots spreading from sub-globose corm R. bulbosus

Ranunculus sceleratus L.

Cursed Buttercup

(Cursed Crowfoot)

Ivy Neck. Infrequent along Cheston Creek drainage and near artificial pond at source of the drainage. Canopy of Platanus occidentalis, Diospyros virginiana, Liquidambar styraciflua. Higman 877, II57.

R. abortivus L.

Littleleaf Buttercup

(Small-flowered Crowfoot)

Java Farm. Two stations, both shaded and apparently well-drained; at north boundary west of new entrance gate, and along cut bank of old Muddy Creek Road. Higman 580,605 .

R. bulbosus L.

St. Anthony's Turnip

(Common Buttercup)

Java Farm \& Ivy Neck. Moderately abundant along dirt roads, and in old fields not yet swamped by Rhus radicans. Prefers moderately dry, sunny or partly shaded areas. Higman 627, II47, II48. 
Thalictrum dioicum L.

Ivy Neck. One station: steep bank overlooking Rhode River, slightly above Sand Point. Hectare 4749. Higman Ior2.

\section{Thalictrum}

(Anemonella)

\section{Thalictrum thalictroides (L.) Eames \& B. Boivin}

Rue Anemone

(Anemonella thalictroides (L.) Spach.)

Ivy Neck. One station in poorly-drained forest of central Scaffold Peninsula. Canopy of Liquidambar styraciflua, Acer rubrum, Liriodendron tulipifera, Fagus grandifolia, Juglans nigra, Quercus alba, Carya cordiformis, etc. Higman 570, 63I.

\section{Anemone \\ Anemone virginiana L. \\ Tall Thimbleweed \\ (Anemone, Wind-flower)}

Java Farm. Infrequent on summit of ridge, northwest of water tank. Canopy of Pinus taeda, Fagus grandifolia, Quercus alba, Q. velutina, Carya tomentosa, etc. Higman 504.

Podophyllum peltatum L.

May Apple

(Mandrake)

Java Farm. Moderately abundant in bottom of Muddy Creek valley, under canopy of Fraxinus americana and Platanus occidentalis. Less frequent on slopes of valley, under canopy of Fagus grandifolia, Quercus alba, Liriodendron tulipifera, etc. Associate species: Claytonia virginica, Smilacina racemosa, Viola papilionacea.

Ivy Neck. Moderately abundant in valley of Scaffold Creek drainage, and less frequently in central Scaffold Peninsula. Canopy of Liquidambar styraciflua, Acer rubrum, Liriodendron tulipifera, Platanus occidentalis, etc. Higman 628. 


\section{MAGNOLIACEAE}

\section{Liriodendron}

Liriodendron tulipifera L.

Tuliptree

(Tulip-tree, Yellow-poplar)

Java Farm and Ivy Neck. Infrequent to moderately abundant in a variety of mature hardwood canopies. Seems to prefer moderately moist upland, sides of stream valleys, etc. Associate species: Quercus alba, Fagus grandifolia, Acer rubrum, Carya cordiformis, Quercus velutina, Cornus florida. Higman 266, ІІ22.

\section{ANNONACEAE}

Asimina

Asimina triloba (L.) Dunal

Pawpaw

(Asimina triloba (L.) Duval)

(Papaw)

Ivy Neck. Infrequent in mixed hardwood forests of Scaffold and Cheston peninsulas. Small clump between cornfield and Sand Point salt marsh. Higman 573.

\section{LAURACEAE}

I. Leaves usually with I or more lobes; flowers in peduncled corymbose racemes, appearing with the leaves; fruit a blue drupe, its pedicel thickened at the end

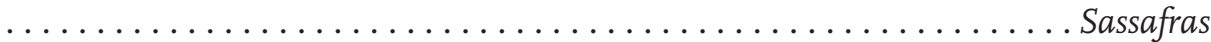

I. Leaves not lobed; flowers in sessile clusters, appearing before the leaves; fruit a red drupe, its pedicel not thickened . . . . . . . . . . . . Lindera

\section{Sassafras}

Sassafras albidum (Nutt.) Nees.

Sassafras

Java Farm. Moderately abundant in old fields and along fences. Associate species: Acer rubrum, Quercus palustris, Liquidambar styraciflua. Infrequent in mature mixed hardwood forest.

Ivy Neck. Infrequent at margins of fields. Higman 382, 584 .

\section{Lindera}

Lindera benzoin (L.) Blume

Northern Spicebush

(Lindera benzoin (L.) Blume, L. benzoin, var. pubescens

(Spice-bush)

(Palmer \& Steyerm.) Rehd.)

Java Farm. Abundant in valley bottom of Muddy Creek. Canopy of Fraxinus americana and Platanus occidentalis. Associate species: Cornus florida, Rubus sp. Infrequent to moderately abundant in forested valleys of tributaries to Muddy Creek. Higman 73, 559, 583, 632. 


\section{PAPAVERACEAE}

\section{Sanguinaria}

Sanguinaria canadensis L.

Bloodroot

Java Farm. One station: along old entrance road under canopy of mature Fagus grandifolia, Quercus alba, Liriodendron tulipifera, etc. Associate species: Dentaria laciniata, Dentaria heterophylla, Claytonia virginica, Viola papilionacea, Desmodium spp., etc. Higman 560.

\section{BRASSICACEAE (CRUCIFERAE)}

I. Petals yellow or orange.

2. Leaves all simple, not deeply divided. Expected (Erysimum, Rorippa, Conringia, Camelina).

2. Leaves, at least the lower, deeply divided, lyrate, or pinnate.

3. Petals I to $2 \mathrm{~cm}$ long. Expected (Raphanus, Brassica).

3. Petals 0.5 to Io $\mathrm{mm}$ long.

4. Cauline leaves clasping the stem by pinnatifid bases ....... Barbarea

4. Cauline leaves not clasping, or if so their bases entire or shallowly dentate.

5. Sepals erect at anthesis; petals 6 to Io $\mathrm{mm}$ long; ovary and fruit beaked. Expected (Brassica).

5. Sepals spreading at anthesis; petals shorter; ovary and fruit beakless. 6. Dry habitat; sepals and ovary linear. (Also, terminal segment of the pinnatifid cauline leaves bluntly to sharply serrate; flowers

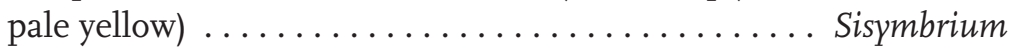

6. Damp to aquatic habitat; sepals and ovary ovate to elliptic. Expected (Rorippa).

I. Petals white, purple, or pink.

7. Petals purple to pink; leaves palmately 3 -divided; plant perennial with fleshy rhizomes; forest habitat . . . . . . . . . . . . . . . Dentaria

7. Petals white; leaves simple or pinnate; plant usually annual or biennial without rhizomes; roadside and field habitat. (Raphanus, above, may have whitish to pale purple petals, lyrate leaves).

8. Leaves simple, not deeply divided.

9. Basal rosettes present. (Lepidium may have a false rosette of cauline leaves).

ı. Cauline leaves absent; flowering stem a scape. (Also, petals cleft to

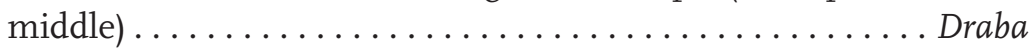

Io. Cauline leaves present.

II. Leaves sagittate at base. Expected (Capsella, Arabis). 
II. Leaves not sagittate at base. (Also, ovary linear; petals spatulate; plant annual with slender taproot) . . . . . . . . Arabidopsis 9. Basal rosettes absent.

I2. Plants halophytic, succulent $\ldots \ldots \ldots \ldots \ldots \ldots$ Cakile

I2. Plants not halophytic or succulent; old-field habitat.

I3. Cauline leaves clasping the stem, sagittate.

I4. Ovary notched at summit; style absent or very short.

I5. Plant pubescent; petals I.5 $\mathrm{mm}$ long ....... Lepidium

I5. Plant glabrous; petals 2-4 mm long. Expected (Thlaspi).

I4. Ovary not notched, but subglobose or cordate; style filiform. Expected (Cardaria).

I3. Cauline leaves not clasping.

I6. Ovary short, lanceolate to ovate; petals I to $2 \mathrm{~mm}$ long or absent ..................... Lepidium

I6. Ovary long, linear or cylindric.

I7. Cauline leaves deltoid-ovate, cordate, long-petioled; plant onion-scented. Expected (Alliaria).

I7. Cauline leaves tapering to base, sessile or nearly so; plant odorless. (Also, blades of petals not raised above sepals; cauline leaves blunt-toothed) ...... Cardamine

8. Leaves deeply pinnatifid, lyrate, or pinnate.

I8. Petals I to $2 \mathrm{~cm}$ long. Expected (Cardamine, Raphanus).

I8. Petals not over $8 \mathrm{~mm}$ long, or absent.

I9. Ovary lance-oblong to round or obcordate. Expected (Teesdalia, Lepidium, Coronopus, Armoracia).

I9. Ovary slenderly linear.

20. Wet or aquatic habitat; petals 4 to $5 \mathrm{~mm}$ long..... Nasturtium

20. Dry habitat; petals shorter ............. Cardamine

Barbarea

I. Uppermost leaves coarsely and irregularly dentate; lyrate lower leaves with I to

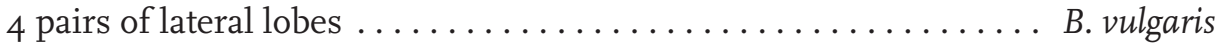

I. Uppermost leaves lyrate-pinnatifid; lyrate lower leaves with io to 20 lateral lobes

B. verna

Barbarea vulgaris W.T. Aiton

(Barbarea vulgaris R. Br.)
Garden Yellowrocket

(Winter Cress)

Java Farm. Moderately abundant in old fields, in areas not yet covered by Rhus radicans and Lonicera japonica. Also along roadsides and around headquarters. Associate species: Draba verna, Stellaria media, Arabidopsis thaliana, Cerastium vulgatum.

Ivy Neck. Moderately abundant in cultivated fields. Same associate species as Java Farm. Higman 596. 
Java Farm and Ivy Neck. Moderately abundant. Associated with B. vulgaris above. Higman 575, 6I7, I082.

Sisymbrium officinale (L.) Scop.

Sisymbrium

(Sisymbrium officinale Scop., var. leiocarpum DC.)
Hedgemustard

(Hedge Mustard)

Ivy Neck. One station: pasture behind mule shed, north of Cheston Creek drainage. Higman 724 .

\section{Cardamine \\ (Dentaria)}

I. Bracts of inflorescence more or less hirsute; flowering stem usually without a

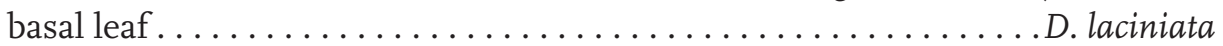

I. Bracts of inflorescence glabrous (occasionally densely hirsute); flowering stem with basal leaf; leaves violet beneath . ............ heterophylla

NOTE: These species are difficult to distinguish, may hybridize.

Cardamine concatenata (Michx.) Sw.

Cutleaf Toothwort

(Dentaria laciniata Muhl.)

(Toothwort)

Java Farm. Infrequent, along old entrance road under mature canopy of Fagus grandifolia, Quercus alba, Liriodendron tulipifera, etc. Associate species: Claytonia virginica, Sanguinaria canadensis, Viola papilionacea, Smilacina racemosa, etc. Higman Io8I.

C. angustata O.E. Schulz

Slender Toothwort

(D. heterophylla Nutt.)

(Toothwort)

Java Farm. Infrequent, associated with D. laciniata. Higman 56I.

\section{Draba}

Draba verna L.

Spring Draba

(Whitlow Grass)

Ivy Neck. Abundant in cultivated fields and along tractor trails, Associate species: Cerastium vulgatum, Barbarea vulgaris, Arabidopsis thaliana, etc.

Java Farm. Infrequent to moderately abundant. Roadsides and open fields. Higman 555 . 
Java Farm and Ivy Neck. Infrequent to moderately abundant. Same habitat as Draba verna above and associated with it. Higman 582, 624.

Cakile edentula (Bigelow) Hook.

\section{Cakile}

(Cakile edentula (Bigel.) Hook.)

American Searocket

(Sea Rocket)

Ivy Neck. Infrequent to moderately abundant on sandy bars and beaches of Cheston Peninsula. Associated with Spartina alterniflora. Higman 860, 936.

\section{Lepidium}

I. Plant densely short-villous; cauline leaves sagittate-clasping at the base, oblanceolate to obovate in shape, entire or shallowly serrate ........ L. campestre

I. Plant glabrous or nearly so; cauline leaves tapering to base, very variable in shape and serration.

2. Petals equaling or longer than the sepals $\ldots \ldots \ldots \ldots \ldots \ldots$. virginicum

2. Petals shorter than the sepals or wanting. Expected (L. densiflorum).

\section{Lepidium campestre (L.) W.T. Aiton}

Field Pepperweed

(Lepidium campestre (L.) R. Br.)

(Field Cress)

Java Farm. Infrequent along new entrance road. Associate species: Dactylis glomerata, Lepidium virginicum, Melilotus alba, Vicia sativum, Trifolium repens. Higman 637.

L. virginicum $\mathrm{L}$.

Virginia Pepperweed

(Pepper Grass)

Java Farm and Ivy Neck. Infrequent along roads and tractor trails. Associate species: Juncus tenuis, Trifolium repens. Higman 68I, 704, 708, 794.

$\begin{array}{lr}\text { Cardamine hirsuta L. } & \text { Hairy Bittercress } \\ \text { (Bitter Cress) }\end{array}$

$\begin{array}{lr}\text { Cardamine } & \text { Hairy Bittercress } \\ \text { (Bitter Cress) }\end{array}$

Ivy Neck. Infrequent to moderately abundant in cultivated fields. Associate species: Draba verna, Cerastium vulgatum, Stellaria media, etc. Higman 554, 634. 
Java Farm. One station: bank of tidal portion of Muddy Creek at mouth of tributary from springhouse. Canopy of Fagus grandifolia and Quercus velutina. Higman 6r6.

\section{CRASSULACEAE}

\section{Sedum}

Sedum sarmentosum Bunge

\section{Stringy Stonecrop}

(Stonecrop)

Ivy Neck. One station: stone flower box in yard of old house (persistent after cultivation). Higman 72I.

\section{CRASSULACEAE}

(SAXIFRAGACEAE)

Penthorum

Penthorum sedoides L.

Ditch Stonecrop

(put in Crassulaceae by Britton and Brown)

Ivy Neck. One station: mud bottom of dry overflow pond on Rhode River shore of Cheston Peninsula. Associate species: Sparganium eurycarpum. Higman IoI5.

\section{HAMAMELIDACEAE}

\section{Liquidambar}

Liquidambar styraciflua L.

Sweetgum

(Sweet Gum)

Java Farm and Ivy Neck. Infrequent to moderately abundant in canopy and understory of all hardwood forest types. Adventive in abandoned fields, often forming very dense stands. Higman $6_{3}$.

Platanus occidentalis L.

\section{PLATANACEAE}

Platanus

American Sycamore

(Sycamore)

Java Farm. Dominates canopy along upper Muddy Creek near culvert of old entrance road and at source of tributary near junction of old entrance road and springhouse road. Hectares 2466, 2476, and 2497. Associate with canopy dominant Fraxinus alba along Muddy Creek valley bottom. Also one station at main building area. 
Ivy Neck. Dominates canopy along drainage of Cheston Creek estuary. Hectares 4722, 4732, 4752. Co-dominants include Diospyros virginiana and Liquidambar styraciflua. Higman 459.

\section{ROSACEAE}

I. Trees or woody shrubs.

2. Ovary inferior; fruit a pome or berry.

3. Plant not spiny; mature carpels papery.

4. Flowers in racemes; pome small, berry-like .......... Amelanchier 4. Flowers in corymbed or umbellate cymes, very showy; pome large and fleshy (a berry in P. melanocarpa) ................. Pyrus

3. Plant with long spines; mature carpels hard, with I to 5 bony nutlets ....

2. Ovary superior; fruit a follicle, capsule, or drupe.

5. Plant a shrub; fruit dehiscent, a follicle or capsule. (Also, flowers white in dense elongate panicles in species collected; cultivated) . . . . . . . Spiraea

5. Plant a tree; fruit indehiscent, a drupe. (Also, flowers in racemes or corymbs

Prunus

I. Herbs or semi-woody shrubs, the latter thorny.

6. Ovaries partly or entirely enclosed by the calyx.

7. Plant herbaceous.

8. Calyx covered with stiff, hooked bristles; petals 5, yellow; leaves pin-

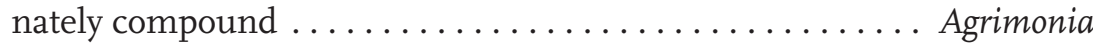

8. Calyx without hooked bristles; petals none, flowers greenish; leaves palmately cleft. Expected (Alchemilla).

7. Plant a thorny shrub. (Also, flowers white and pink, showy; calyx an open cup, ripening as a fleshy lip; leaves pinnately compound). . . . . . . Rosa

6. Ovaries not enclosed by the calyx.

9. Plant a thorny shrub; fruit a cluster of juicy drupelets

Rubus

9. Plant herbaceous; fruit achenes.

Io. Plant prostrate-spreading.

II. Receptacle pulpy, enlarged in fruit (strawberry-like); leaflets 3, digitate.

I2. Petals white; bractlets of calyx similar to the narrow lobes $\ldots \ldots$. Fragaria

I2. Petals yellow; bractlets of calyx much broader than the lobes, 3-toothed. (Also, plant very pilose; stipules 3-divided) ......

........................... Duchesnea

II. Receptacle dry, hairy, not enlarged in fruit; leaflets 5, palmate. (Also, petals yellow) ............... Potentilla ıo. Plant erect. (Also, receptacle hairy). 
I2. Petals yellow; receptacle flatish; leaves oblanceolate to narrowly obovate, coarsely serrate, never lobed ............ Potentilla

I2. Petals white; receptacle conic-cylindric; leaves broadly oblanceolate or rhombic, serrate, the lower often lobed or cleft ....... Geum

\section{Amelanchier}

I. Racemes ascending; sepals divergent in fruit; petals 7-I2 $\mathrm{mm}$ long; leaves blunt or acute at apex, glabrous beneath, round at base ........... A. canadensis

I. Racemes drooping; sepals tightly reflexed in fruit; petals $\mathrm{I}-2 \mathrm{~mm}$ long; leaves acuminate at apex, tomentose beneath, round or cordate at base.... . A. arborea

Amelanchier canadensis (L.) Medik.

(Amelanchier canadensis Medic.)

Canadian Serviceberry (Shad-bush, Service Berry)

Ivy Neck. Two stations: head of Scaffold Creek estuary and east bank of Scaffold Peninsula. Canopy of Quercus alba, Carya tomentosa, Fagus grandifolia, Acer rubrum. Higman 594, 844 .

A. arborea (Michx. f.) Fernald

(A. arborea (Michx. f.) Fern.)
Common Serviceberry (Shad-bush, Service Berry)

Java Farm. One station: Hog Island. Hectare 3592. Canopy of Quercus prinus. Associate species: Rhododendron nudiflora, Nyssa sylvatica, Gaylussacia baccata, Cornus florida. Higman II7.

\section{Photinia, Pyrus, Malus}

I. Leaves glandular along upper midrib; petals less than I $\mathrm{cm}$ long and $7 \mathrm{~mm}$ broad; fruit small and berry-like ................... melanocarpa

I. Leaves not glandular; petals $\mathrm{I}-2 \mathrm{~cm}$ long and $0.8-\mathrm{I} .5 \mathrm{~cm}$ broad; fruit large and fleshy.

2. Leaves and branchlets glabrous; fruit pear-shaped, yellow ...... P. communis

2. Leaves and branchlets white-tomentose; fruit apple-shaped, red .... P. malus

Photinia melanocarpa (Michx.) K.R. Robertson \& Phipps

Black Chokeberry

(Pyrus melanocarpa (Michx.) Willd.)

(Chokeberry)

(Aronia melanocarpa (Michx.) Ell.)

Java Farm. One station on Fox Point, under canopy of Quercus prinus. Also observed on Hog Island. Hectares 3558, 3592. Higman 532.

Pyrus communis L.

Common Pear

(Pear)

Java Farm. Infrequent on steep west-facing slope overlooking meadow, adjacent northern boundary. Persistent after cultivation. Canopy of Juglans nigra. 
Java Farm. Infrequent on south-facing slope overlooking meadow, adjacent northern boundary. Persistent after cultivation. Open canopy also includes Liriodendron tulipifera and Robinia pseudoacacia.

Ivy Neck. Infrequent on borders of cultivated fields. Presumably cultivated. Higman $157,506,578,597,604$.

\section{Crataegus pruinosa (Wendl. f.) K. Koch}

(Crataegus pruinosa (Wendl.) K. Koch)

\section{Crataegus}

Ivy Neck. One station on east shore of Scaffold Creek; canopy of Quercus prinus, Q. velutina, and Carya tomentosa. Another station on east shore of Cheston Creek; small area of Quercus phellos-mixed hardwoods. Higman 656, I073.

\section{Spiraea}

Spiraea prunifolia Siebold \& Zucc. (Spiraea prunifolia Sieb. \& Zucc?)

\author{
Waxyfruit Hawthorn \\ (Hawthorn, Red Haw)
}

Ivy Neck. Two stations near old houses. Hectares 4720, 4658. Persistent after cultivation. Higman 593, II44.

\section{Prunus}

I. Flowers in long racemes $\ldots \ldots \ldots \ldots \ldots \ldots \ldots \ldots \ldots$. serotina

I. Flowers umbellate or corymbed.

2. Ovary and fruit tomentose $\ldots \ldots \ldots \ldots \ldots \ldots \ldots \ldots$. persica

2. Ovary and fruit glabrous, not glaucous ............. P. avium

Prunus serotina Ehrh.

Black Cherry

Java Farm. Infrequent to moderately abundant in old fields and along roadsides. Associate species: Sassafras albidum, Acer rubrum, Quercus palustris, Liquidambar styraciflua, Cornus florida.

Ivy Neck. Infrequent in old fields between east and west forks of Scaffold Creek estuary, in forest at source of Scaffold Creek drainage (east fork), and in forest of central Scaffold Peninsula. Moderately abundant on Cheston Point and in coves behind salt marsh there. Associate species: those at Java Farm plus Robinia pseudoacacia, Rhus typhina, Quercus velutina. Higman I39, 644.

P. persica (L.) Batsch

Peach

Java Farm. One station at main buildings. Hectare 2580. Persistent after cultivation. Higman 598, 867. 
Java Farm. Infrequent along shore of Muddy Creek and Fox Creek estuaries. Associate species not definite, may include Robinia pseudoacacia, Liquidambar styraciflua, Ulmus rubra, Cornus florida.

Ivy Neck. Infrequent along shores of estuaries and at Cheston Point, not associated with Quercus prinus canopy. Also one station (presumably cultivated) beside old house on upper Scaffold Peninsula. Higman 422, 424, 585, 767, II63, II64.

\section{Agrimonia}

I. Larger leaflets of middle and upper leaves lance-acuminate, sharply serrate, villous and copiously white-glandular-dotted beneath. (Also, axis of raceme

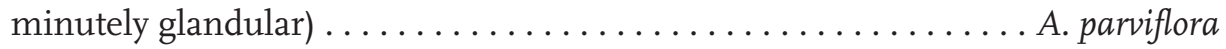

I. Larger leaflets of middle and upper leaves ovate-oblong, coarsely dentate, either villous or glandular-dotted but not both.

2. Axis of raceme minutely glandular, glabrous or nearly so; rachis and lower leaflet surfaces glabrous . . .................. rostellata

2. Axis of raceme glandless, pubescent; rachis and lower leaflet surfaces pubescent.

3. Larger leaflets broadly obtuse at apex; mature hypanthium about $2 \mathrm{~mm}$

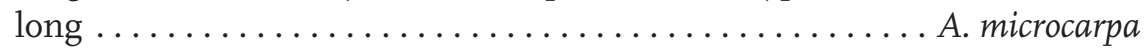

3. Larger leaflets acute to blunt at apex; mature hypanthium about $3 \mathrm{~mm}$ long. Expected (A. pubescens).

\section{Agrimonia parviflora Aiton}

Harvestlice

(Agrimonia parviflora Ait.)

(Agrimony)

Java Farm. Infrequent in grassy fields between south side of main building area and old entrance road. Associate species: Solanum carolinense, Cirsium discolor, Erigeron annuus, Verbascum Thapsus, Asclepias syriaca.

Ivy Neck. One station: drainage into Cheston Creek estuary. Hectare 4743. Canopy of Robinia pseudoacacia, Diospyros virginiana, Platanus occidentalis. Associate species: Elephantopus carolinianus, Cuphea petiolata.

A. rostellata Wallr.

Beaked Agrimony

(Agrimony)

Java Farm. Infrequent along abandoned trail between old entrance gate and Muddy Creek, parallel to north boundary. Canopy of mature Fagus grandifolia, Carya tomentosa, Quercus alba, Liriodendron tulipifera, etc. Higman I079. 
Java Farm. Infrequent along old entrance road between gate and Muddy Creek. Canopy same as for A. rostellata above. Associate species: Lactuca floridana, Eupatorium purpureum, Desmodium paniculatum, Desmodium glutinosum, Elephantopus carolinianus, Polygonum cespitosum var. longisetum. Higman 32.

\section{Rosa}

I. Styles united into a column equaling the stamens.

2. Stipules entire; leaflets 3. Expected (R. setigera).

2. Stipules fringed like a comb; leaflets 7 or 9 R. multiflora

I. Styles free (sometimes partly united) and shorter than the stamens.

3. Sepals persistent in fruit; foliage not glandular-aromatic. Expected ( $R$. cinnamomea).

3. Sepals deciduous in fruit, if persistent, the foliage glandular-aromatic.

4. Mature hips $2 \mathrm{~cm}$ long or more, ellipsoid-ovoid, the achenes often visible through the walls. (Also, leaves glabrous, glandless, not aromatic) ......

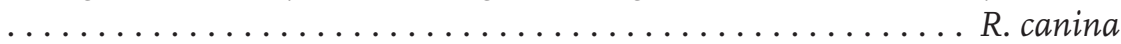

4. Mature hips usually I cm long or shorter (I.5 cm long in R. micrantha), ovoid-subglobose, opaque.

5. Leaves glandular beneath at least, oval-ovate, the teeth glandular or gland-tipped; hips sparsely glandular-hirsute to glabrous; sepals fringed like a comb.

6. Sepals persistent until fruit is entirely ripe (only sure distinction); leaflets strongly glandular above; styles pubescent. Expected (R. eglanteria).

6. Sepals promptly deciduous in fruit; leaflets sparsely glandular above; styles glabrous or nearly so $\ldots \ldots \ldots \ldots \ldots \ldots$. micrantha

5. Leaves glandless, narrowly elliptic-obovate; hips and sepals glandularhirsute; sepals entire or with 2 narrow lateral divisions.

7. Stipules upwardly dilated, the spreading adnate part of the pair 3-IO $\mathrm{mm}$ broad. Expected (R. virginiana).

7. Stipules with parallel sides, the adnate part $0.5^{-2} \mathrm{~mm}$ broad.

8. Sepals entire; leaflets glabrous, or pilose only on veins beneath; cauline thorns stout and recurved ........... palustris

8. Sepals with 2 narrow lateral divisions; leaflets pilose beneath; cauline thorns slender and straight $\ldots \ldots \ldots \ldots \ldots$. Carolina

Rosa multiflora Thunb.

Multiflora Rose

(Rose)

Java Farm and Ivy Neck. Infrequent, usually on banks of streams and in nearby old fields. Associate species: Gratiola virginiana, Trifolium repens, Tripsacum dactyloides, Panicum clandestinum, Viburnum prunifolium, Rhus typhina, Celastrus scandens. Higman 221, 328, 680, 703, 727. 
Ivy Neck. One station: steep bluff above sandy shore of Rhode River on Cheston Peninsula, north of Sand Point. Associate species: Amorpha frutescens, Rhus typhina, Verbascum thapsus, Daucus carota, Atriplex patula, Plantago lanceolata, Juncus tenuis, Solidago sempervirens, Cirsium discolor, Arctium minus. Higman 368, II62.

R. micrantha Borrer ex Sm.

Smallflower Sweetbrier

(R. micrantha Sm.)

(Sweetbrier)

Java Farm. Infrequent in old fields. Associate species: Rosa palustris, Solidago altissima, Rhus radicans. Higman 377, 76r.

R. palustris Marshall

Swamp Rose

(R. palustris Marsh.)

Java Farm. Infrequent along shores of streams and estuaries, landward margin of salt marsh on tidal portion of Muddy Creek, and scattered along Fox Creek drainage and adjacent fields. Associate species: Salix nigra, Rosa micrantha, Myrica cerifera, Viburnum prunifolium. Higman 291, 368, 379, 466, 861.

R. carolina L.

Carolina Rose

(Rose)

Ivy Neck. One station: east shore of Scaffold Peninsula. Canopy of mature Quercus prinus, Quercus alba, Carya tomentosa, Acer rubrum, Fagus grandifolia. Higman 846.

\section{Rubus}

I. Flowers $3-4 \mathrm{~cm}$ broad, with spreading petals; fruit often drying in the receptacle without falling; leaves pale green beneath, pilose on both sides; stems and petioles very bristly $\ldots \ldots \ldots \ldots \ldots \ldots \ldots \ldots \ldots \ldots \ldots \ldots \ldots \ldots$ hispidus

I. Flowers much smaller, with calyx lobes exceeding petals; fruit readily falling from receptacle; leaves densely white-woolly beneath, nearly glabrous above; stems and petioles pubescent or glabrous.

2. Stems, petioles, pedicels, and calyx with dense red glandular hairs; leaflets

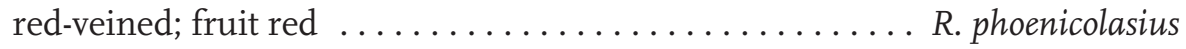

2. Stems glabrous but often very glaucous; petioles and pedicels slightly pilose with non-glandular hairs; calyx tomentose; leaflets green-veined; fruit purple-

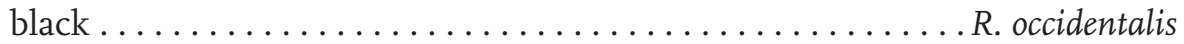

Rubus hispidus L.

Bristly Dewberry

(Dewberry, Bramble)

Java Farm. One station: border of mixed hardwood forest at end of road to springhouse. Hectare 3427. 
Ivy Neck. One station: prostrate in cornfield on Scaffold Peninsula and in adjacent forest.

(May be infrequent to moderately abundant at forest borders.) Higman 658 .

R. phoenicolasius Maxim.

Wine Raspberry

(Wineberry)

Java Farm. One station: near northeast border fence. Hectare 2550. Summit of steep west-facing slope (old orchard); dense canopy of Juglans nigra, dense ground layer of Lonicera japonica. Also in small ravine at base of slope, adjoining meadow. Higman 78I.

R. occidentalis L.

Black Raspberry

Java Farm. Dominant in many old fields, forming dense clumps. Seems to compete well with Campsis radicans and Lonicera japonica. Also scattered in grassy areas. Higman 702, 648.

Fragaria virginiana Duchesne

Fragaria

Virginia Strawberry

(Strawberry)

Java Farm. Three stations: grassy triangle at entrance gate, washed-out culvert on upper Fox Creek, and open glade at end of springhouse road. All sites moderately moist, moderately sunny, and moderately free of Rhus radicans and Lonicera japonica. Associated with Viola spp., Duchesnea indica, assorted grasses. Higman 622.

Duchesnea indica (Andrews) Teschem.

(Duchesnea indica (Andr.) Focke.)

\section{Duchesnea}

Java Farm. Moderately abundant to abundant in same habitat as Fragaria virginiana above. Also found in bottom of Muddy Creek valley, under canopy of Fraxinus americana and Platanus occidentalis; associate species there include Ranunculus sceleratus and Allium vineale.

Ivy Neck. Moderately abundant along Cheston Creek drainage. Canopy of mature Platanus occidentalis, Robinia pseudoacacia. Higman I77, 313, 609, 783.

\section{Potentilla}

I. Stem erect, 2-9 dm high.

2. Leaves digitately 5-to 7-foliate; teeth of larger leaflets narrowly deltoid, ascending toward the apex; petals notched at apex; flowers 15 to $25 \mathrm{~mm}$ broad

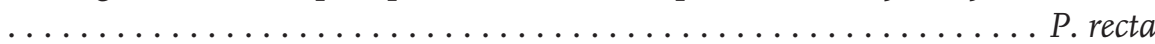

2. Leaves palmately 3 -foliate; teeth of larger leaflets broadly acute, spreading; petals only slightly notched at apex; flowers 3 to $10 \mathrm{~mm}$ broad .... P. norvegica 
I. Stem prostrate-trailing.

3. Rhizome short and blunt, max. $2 \mathrm{~cm}$ long; stipules of basal leaves with oblong-lanceolate flat auricles; leaves small at anthesis ..... P. canadensis

3. Rhizome irregularly enlarged, max. $8 \mathrm{~cm}$ long; stipules of basal leaves with linear-lanceolate inrolling auricles; leaves expanded at anthesis . . . . P. simplex

(NOTE: These last two species look very similar in the field and on herbarium sheets, and distinguishing characters cannot be fully trusted.)

Potentilla recta L.

Sulphur Cinquefoil

(Five-finger)

Java Farm \& Ivy Neck, One station each: both on open, unshaded roadsides. Higman 720.

P. norvegica L.

Norwegian Cinquefoil

(Five-finger)

Java Farm. One station at border of parking lot, main building area. Hectare 2580.

Ivy Neck. One station: near old barn on south side of artificial pond at source of Cheston Creek drainage. Hectare 4723. Associate species: Ipomoea hederacea, Datura stramonium, Cyperus strigosus, Abutilon theophrasti, Eleusine indica, Polygonum persicaria. Higman 932, 998.

P. canadensis L.

Dwarf Cinquefoil

(Five-finger)

Java Farm. One station: near old entrance gate. Hectare 2463. Canopy of mature Fagus grandifolia, Quercus alba, Quercus velutina, Liriodendron tulipifera, Carya tomentosa, etc. Higman 664.

P. simplex Michx.

Common Cinquefoil

(Five-finger)

Java Farm. One station: along old Muddy Creek Road. Same habitat as P. canadensis above. Hectare 2472. Higman 665.

\section{Geum}

Geum canadense Jacq.

White Avens

Java Farm. Moderately abundant along new entrance road, especially near culvert of Fox Creek drainage. Associate species: Panicum clandestinum, Duchesnea indica, Agrimonia microcarpa, Lonicera japonica. Site is partly shaded by Juglans nigra and Liquidambar styraciflua. Less frequent in forest north of Fox Creek marsh and in Muddy Creek valley near old entrance road. Both areas have dense, mature canopy and a dense ground layer of Lonicera japonica. Associate species at both locations: Elephantopus carolinianus. Higman I84, 248, 830. 


\section{FABACEAE \\ (LEGUMINOSAE)}

I. Stamens completely separate, (monadelphous at base in Amorpha).

2. Stamens inserted on the calyx.

3. Herbs; flowers yellow; petioles with I or more glands

Cassia

3. Trees; flowers white or pink; petioles without basal glands.

4. Plants thorny. Expected (Gleditsia).

4. Plants thornless.

5. Leaves bipinnate, with ovate leaflets; flowers white, not papilionaceous; legumes thick and woody, I dm or more in length . . . . . . . .

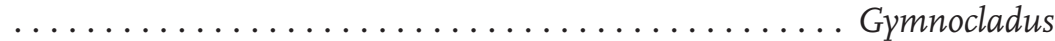

5. Leaves simple, cordate; flowers pink, papilionaceous, appearing before the leaves; legumes not woody, approx. $5-7 \mathrm{~cm}$ long ...... Cercis

2. Stamens inserted on the corolla.

6. Shrubs; sandy shoreline habitat; leaves with 13 to 35 leaflets; corolla of I violet petal; legumes resin-dotted . . . . . . . . . . . Amorpha

6 . Herbs; inland habitat; leaves with 3 leaflets; corolla of 5 yellow petals (papilionaceous); legumes not resinous . . . . . . . . . . . Baptisia

I. Stamens monadelphous or diadelphous. Herbs, except Robinia. (Wisteria and Cytisus omitted).

7. Anthers of 2 forms; legume dehiscent. (Also, stamens monadelphous). Expected (Lupinus).

7. Anthers uniform. (Stylosanthes has 2 forms but legume is indehiscent).

8. Leaflets denticulate or serrulate.

9. Inflorescence a head; petals persistent in fruit ......... Trifolium

9. Inflorescence a raceme or spike; petals deciduous in fruit.

Io. Legume straight; plant about I $m$ tall ............... Melilotus

Io. Legume curved; plant (in species collected) low and spreading ...

8. Leaflets entire.

II. Trees, thorny. (Also, 3 to Io pairs of leaflets) $\ldots \ldots \ldots$. . Robinia

II. Herbs, thornless.

I2. Plant not a vine, without tendrils nor twining. (2 species of Lespedeza are prostrate and spreading).

I3. Leaflets 5 or more, up to approx. 25; legume dehiscent. Expected (Aeschynomene, Tephrosia).

I3. Leaflets 3; legume indehiscent.

I4. Leaflets stipellate (with appendages like stipules); calyx 2-lipped; flowers purple to pink; legume adhesive . . . . . .

Desmodium 

yellow or whitish; legume not adhesive.

I5. Flowers whitish with purple markings; stamens diadelphous; calyx persistent in fruit. (Some species have apetalous flowers also)......... Lespedeza

15. Flowers yellow; stamens monadelphous (2 kinds of anthers); calyx deciduous in fruit ...... Stylosanthes

I2. Plant a vine, trailing or twining, may have tendrils.

I6. Tendrils terminating the pinnate leaves; leaflets so or more

I6. Tendrils absent; leaves odd-pinnate, with 3 to 9 leaflets.

I7. Leaflets usually 5 to 9 , ovate-lanceolate ......... Apios

I7. Leaflets 3.

I8. Corolla yellow. Expected (Rhynchosia).

I8. Corolla blue or purple to white.

I9. Style not bearded.

20. Flowers both petaliferous and apetalous, the latter attached to roots; calyx of petaliferous flowers without small bracts at base of pedicel; calyx teeth subequal ....... Amphicarpa

20. Flowers all petaliferous; calyx with small bracts at base of pedicel; calyx 2-lipped, deeply cleft. Expected (Pueraria).

I9. Style bearded.

2I. Style bearded at summit around stigma. Expected (Centrosema).

2I. Style bearded along upper surface.

22. Flowers numerous in racemes or panicles; keel spirally coiled ...... Phaseolus

22. Flowers I to few in heads or short racemes; keel curved but not spirally coiled. 23. Flowers 4-6 cm long. Expected (Clitoria).

23. Flowers $0.6-2.5 \mathrm{~cm}$ long. (Also, keel long and blackened; flowers creamy with pink tinge; legume linear, flat) .............. Strophostyles 


\section{Chamaecrista}

(Cassia)

Chamaecrista fasciculata (Michx.) Greene var. fasciculata

Partridge Pea

(Cassia fasciculata Michx.)

Java Farm. Infrequent along Fox Point Road. Associate species: Daucus carota, Verbascum thapsus, Plantago major, Prunella vulgaris, Ambrosia artemisiifolia, Erigeron canadensis.

Ivy Neck. Infrequent on tractor trails and field borders. Higman I6.

Gymnocladus dioicus (L.) K. Koch

$$
\text { Gymnocladus }
$$

Kentucky Coffeetree

(Kentucky Coffee-tree)

Ivy Neck. One station: large tree by parking area, north of entrance road. Hectare 4720. Probably cultivated. Higman I047, I053.

\section{Cercis}

Cercis canadensis L.

Eastern Redbud

(Redbud)

Ivy Neck. Infrequent in forest of central Scaffold Peninsula. Canopy of Quercus alba, Quercus velutina, Nyssa sylvatica, Liriodendron tulipifera, Carya tomentosa, etc. Understory includes Rhus typhina. Higman 595.

Amorpha fruticosa L.

$$
\text { Amorpha }
$$

False Indigo Bush

(False Indigo)

Ivy Neck. Infrequent along landward margin of sandy beach, at shore of Rhode River. Associate species: Baccharis halimifolia. Higman Io63.

Baptisia tinctoria (L.) R. Br.

Baptisia
Horseflyweed

(Wild Indigo)

Java Farm. One station: Fox Point, at end of road. Hectare 3558. Canopy of mature Quercus prinus, Quercus alba. Associate species: Liatris graminifolia. Higman 43I.

I. Flowers sessile in head.

\section{Trifolium}

2. Heads cylindric, not subtended by opposite leaves; flowers pale gray; calyx long-villous $\ldots \ldots \ldots \ldots \ldots \ldots \ldots \ldots \ldots \ldots \ldots \ldots \ldots \ldots \ldots \ldots \ldots \ldots \ldots$. arvense 
2. Heads subglobose, subtended by opposite leaves; flowers pink or reddish; calyx densely short-pilose $\ldots \ldots \ldots \ldots \ldots \ldots \ldots \ldots \ldots \ldots$. pratense

I. Flowers pedicellate in head.

3. Flowers yellow, becoming brown with age ........... procumbens

3. Flowers white to reddish-pink.

4. Stems repent or creeping, the peduncles ascending; flowers white .....

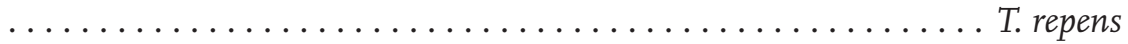

4. Stems erect or ascending; flowers pink or reddish ...... T. hybridum

Trifolium arvense $\mathrm{L}$.

Rabbitfoot Clover

(Rabbit-foot Clover)

Ivy Neck. Infrequent along tractor trail beside grassy abandoned field. Associate species: Hypericum perforatum, Festuca elatior, Trifolium hybridum, Rudbeckia serotina. Higman 8I3.

T. pratense L.

Red Clover

Ivy Neck. Moderately abundant in central part of grassy abandoned field. Associate species: Trifolium hybridum, Verbascum blatteria, Oenothera fruticosa. Higman 545, 695.

T. pratense $\mathrm{L}$.

Red Clover

(T. pratense, var. sativum (Mill.) Schreb.)

Java Farm. Infrequent along new entrance road. Associate species: Vicia sativa, Melilotus alba. Higman 203.

T. campestre Schreb.

Field Clover

(T. procumbens L.)

(Hop Clover)

Java Farm. One station: south-facing slope adjacent north border, overlooking meadow. Hectare 2458. Open canopy of Pyrus malus, Robinia pseudoacacia, Liriodendron tulipifera. Fairly dense Rhus radicans and Lonicera japonica. Higman iıI9.

T. repens L.

White Clover

Java Farm, Infrequent to moderately abundant along old entrance road and road to springhouse. Higman 673, 705, 706.

T. hybridum L.

Alsike Clover

Ivy Neck. Moderately abundant in grassy abandoned field. Associated with T. pratense above. Higman 687, 689, 8I7. 


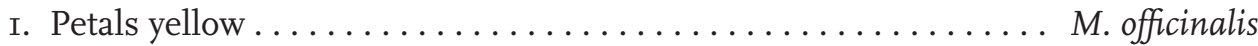

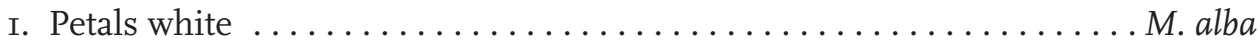

Melilotus officinalis (L.) Lam. Sweetcover

(Yellow Sweet Clover)

Java Farm. Moderately abundant around main buildings and along sunny roadsides.

Ivy Neck. Infrequent, around farm buildings. Higman 5II, 700.

M. officinalis (L.) Lam.

Sweetclover

(M. alba Desr.)

(White Sweet Clover)

Java Farm and Ivy Neck. Same habitat as M. officinalis above; somewhat more abundant. Associate species: Cirsium discolor, Osclepias syriaca, Verbascum thapsus. Higman 36r.

Medicago lupulina L.

\section{Medicago}

Java Farm. One station: weedy lawn beside parking area at main buildings. Hectare 2580. Associate species: Melilotus alba. Higman 70I,

Robinia pseudoacacia L.

\section{Robinia}

Black Locust

(Yellow Locust (L))

Java Farm and Ivy Neck. Infrequent to moderately abundant in old fields and immature forest, often associated with Liquidambar styraciflua. Absent to infrequent in mature hardwood forest. Formerly cultivated at Java Farm, on south- and westfacing slopes overlooking meadow. Higman 264, 696, 1046.

\section{Desmodium}

I. Stamens monadelphous; stipe of loment (legume) equal to or exceeding the pedicel, more than 3 times as long as calyx.

2. Inflorescences axillary and terminal; flowers white. Expected (D. pauciflorum).

2. Inflorescences terminal only; flowers rose to purple. (Also, flowering stem leafless or nearly so).

3. Flowering stem leafless at base; leaves at apex of a sterile stem. Expected (D. nudiflorum).

3. Flowering stem arising from a basal whorl of leaves ....... D. glutinosum

I. Stamens diadelphous (9 and I); stipe of loment shorter than the pedicel, not over twice as long as calyx.

4. Stipules ovate-attenuate, cordate at base, conspicuous. Expected (D. ochroleucum, D. canescens). 
4. Stipules linear to lance-ovate-attenuate, often deciduous.

5. Loments with I to 3 articles. (Also, flowers not longer than $6 \mathrm{~mm}$; bracts not longer than $3 \mathrm{~mm}$ ) Expected (D. rigidum, D. ciliare, D. marilandicum).

5. Loments with 4 or more articles (occasionally only 3 ).

6. Leaflets linear-lanceolate to lance-ovate, not ciliate, nearly glabrous; stem glabrous or nearly so .............. D. paniculatum

6. Leaflets rhombic to ovate, ciliate, pilose on both surfaces; stem puberulent and/or pilose.

7. Venation of leaflets conspicuously reticulate.

8. Flowers 6.5-13.5 $\mathrm{mm}$ long; primary bracts up to I2 $\mathrm{mm}$ long; loments sessile or nearly so . . . . . . . canadense

8. Flowers and bracts smaller; loments on stipes.

9. Leaflets tomentose beneath, velvety to touch, pilose above. Expected (D. viridiflorum).

9. Leaflets appressed-pilose on both surfaces...... D. glabellum

7. Venation of leaflets inconspicuous.

Io. Plant prostrate. (Also, stipules ovate-to lance-attenuate). Expected (D. humifusum).

Io. Plant erect. (Also, stipules linear-to lance-attenuate) . . . . . . . ............................. perplexum

Desmodium glutinosum (Muhl. ex Willd.) Alph. Wood

(Desmodium glutinosum (Muhl.))

Pointedleaf Ticktrefoil

(Wood Tick Trefoil)

Java Farm. Infrequent to moderately abundant along old entrance road. Canopy of mature Fagus grandifolia, Quercus alba, Liriodendron tulipifera, etc. Associate species: Agrimonia microcarpa, Lactuca floridana var. villosa, Eupatorium purpureum, Elephantopus carolinianus, Smilacina racemosa, Tovara virginiana, Dentaria laciniata, etc. Higman 34, 36.

D. paniculatum (L.) DC.

Panicledleaf Ticktrefoil (Tick Trefoil)

Java Farm. Infrequent along old entrance road. Associated with D. glutinosum above. Higman 33 .

D. canadense (L.) DC.

Showy Ticktrefoil

(Tick Trefoil)

Java Farm. Infrequent along old entrance road. Associated with D. glutinosum above. Higman 40. 
D. glabellum (Michx.) DC.

Dillenius' Ticktrefoil

(Tick Trefoil)

Java Farm. Infrequent along old entrance road. Associated with D. glutinosum above. Higman 39.

D. perplexum B.G. Schub.

Perplexed Ticktrefoil

(D. perplexum Schub.)

(Tick Trefoil)

(not in Britton and Brown)

Java Farm. One station in large field south of old entrance road and east of road to springhouse. Hectare 34I9. Rhus radicans and Solidago altissima dominate. Associate species: Lespedeza cuneata, Eupatorium serotinum, Cyperus strigosus, Clematis virginiana, Daucus carota.

Ivy Neck. One station in fallow field between entrance road and Scaffold Creek drainage. Hectare 4668. Associate species: Setaria lutescens, Eupatorium serotinum, Echinochloa pungens, Strophostyles umbellata. Higman II9, 834.

\section{Kummerowia,}

Lespedeza

I. Stipules and bracts broad and scarious; calyx lobes broad; annuals. (Stem prostrate in species collected).

2. Flowers and fruits in tiny axillary clusters. Expected (L. striata).

2. Flowers and fruits in long, bracted spiciform racemes ....... L. stipulacea

I. Stipules and bracts narrow to awl-shaped; calyx lobes narrow; perennials.

3. Petaliferous flowers in racemes or panicles; apetalous ones present; calyx shorter than the legume, flowers violet.

4. Peduncles of petaliferous flowers 2 to 4 times as long as the subtending leaves.

5. Stems erect or ascending. Expected (L. violacea).

5. Stems prostrate, spreading.

6. Stems and leaf lets, densely pubescent ......... L. procumbens

6. Stems and leaflets glabrous or nearly so .......... repens

4. Peduncles of petaliferous flowers equal to or shorter than the subtending leaves. Expected (L. nuttallii, L. virginica, L. intermedia).

3. Petaliferous flowers in heads, spikes, or axillary racemes; apetalous ones absent (except in L. cuneata, in which the calyx equals the legume); flowers white with purple markings.

7. Inflorescences at summit of stems and branches; calyx very pilose. Expected (L. capitata, L. hirta).

7. Inflorescences axillary along erect branches; calyx nearly glabrous . . . . .

L. cuneate 
Ivy Neck. One station: on dirt road leading to abandoned wheat field. Also old house by tobacco barn. Higman Io22. Hectares 4658, 4740.

Lespedeza procumbens Michx.

Trailing Lespedeza

(Bush Clover)

Java Farm. One station: level bank above upper Fox Creek; open area dominated by Rubus spp. and Tripsacum dactyloides. Higman 323. Hectare 2593.

L. repens (L.) W.P.C. Barton

Creeping Lespedeza

(L. repens (L.) Bart.)

(Bush Clover)

Java Farm. One station: south-facing slope adjacent north boundary, overlooking meadow. Open canopy of Pyrus malus, Liriodendron tulipifera, and Robinia pseudoacacia. Dense ground cover of Lonicera japonica and Rhus radicans except in a few spots like this station. Higman i63. Hectare 2459.

L. cuneata (Dum. Cours.) G. Don

Sericea Lespedeza

(L. cuneata (Dumont) G. Don)

(Bush Clover)

Java Farm. Infrequent to moderately abundant in old fields dominated by Rubus spp., Solidago altissima, Campsis radicans, Lonicera japonica, Rhus radicans, etc. Higman I23, 372 .

\section{Stylosanthes}

Stylosanthes biflora (L.) Britton, Sterns \& Poggenb.

Sidebeak Pencilflower

(Stylosanthes biflora (L.) BSP.)

(Pencil-flower)

Java Farm. Collected by H. David Hammond.

Vicia

I. Peduncle very short or wanting. (Also, flowers purple).

2. Flower I.8-3 cm long; mature legumes brown, terete . . . . . . . . V. sativa

2. Flower I-I.8 cm long; mature legumes black, somewhat flattened . . . . . .

I. Peduncle equal to or longer than the leaflets.

3. Flowers i to $8,3-8 \mathrm{~mm}$ long.

4. Legume beakless, not oblique ............... V. tetrasperma

4. Legume beaked, oblique at the tip. Expected ( $V$. hirsuta).

3. Flowers 3 to 40 or more, $0.8-2 \mathrm{~cm}$ long (if less than 8 in number, then I.5-2 cm long). 
5. Calyx teeth deltoid; flowers scattered in racemes. Expected (V. caroliniana).

5. Calyx teeth lance-attenuate to linear; flowers strongly overlapping.

6. Plant glabrous or appressed-pilose. Expected (V. dasycarpa).

6. Plant villous, the lower calyx teeth ciliate .............. V. villosa

Vicia sativa $\mathrm{L}$.

Garden Vetch

(Vetch)

Java Farm. Infrequent along new entrance road. Associate species: Melilotus alba, Trifolium pratense, Lepidium campestre, Ranunculus bulbosus. Higman 635.

V. sativa L. ssp. nigra (L.) Ehrh.

Garden Vetch

(V. angustifolia Reichard)

(Vetch)

Java Farm. Infrequent along old entrance road, near main building area. Lonicera japonica and Rhus radicans dominant. Higman ${ }_{11} 65$.

V. tetrasperma (L.) Schreb.

Lentil Vetch

(V. tetrasperma (L.) Moench.)

(Vetch)

Java Farm. Infrequent along old entrance road, near main building area. Higman II66.

V. villosa Roth subsp. varia (Host) Corb.

Winter Vetch

( $V$. villosa Roth, var. glabrescens)

(Vetch)

(variety not in Britton and Brown)

Ivy Neck. One station in grassy field near parking area and old house. Hectare 4720. Higman 719.

Java Farm. One station beside entrance road. Higman I246.

Apios

Apios americana Medik.

Groundnut

(Apios americana Medic.)

(Wild Bean, Ground-nut)

Java Farm. One station: salt marsh along tidal portion of Muddy Creek, upstream from Hog Island marsh. Hectare 3540. Associate species: Spartina alterniflora, Pluchea camphorata, Kosteletzkya virginica. Higman 283.

\footnotetext{
Amphicarpaea

(Amphicarpa)

Amphicarpaea bracteata (L.) Fernald

American Hogpeanut

(Amphicarpa bracteata (L.) Fern.)

(Hog Peanut)

Java Farm. Infrequent along banks of Muddy Creek (fresh-water). Canopy of Fraxinus americana; understory of Lindera benzoin. Higman 92, I044.
} 
Java Farm. One station: valley of Muddy Creek, upstream from culvert at old entrance road, near Phalaris arundinacea meadow. Hectare 2467. Canopy of Platanus occidentalis, Fagus grandifolia, Quercus alba. Understory of Lindera benzoin. Ground layer of Lonicera japonica. Higman I78.

\section{Strophostyles}

I. Principal leaflets ovate, may be somewhat 3-lobed, acuminate; stem glabrous or

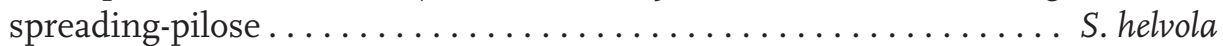

I. Principal leaflets very narrowly ovate, unlobed; stem retrorsely pilose for I-2 $\mathrm{dm}$ back from tip only $\ldots \ldots \ldots \ldots \ldots \ldots \ldots \ldots \ldots \ldots \ldots \ldots$. umbellate

Strophostyles helvola (L.) Elliott

Amberique-bean (Strophostyles helvola (L.) Ell.)

(Wild Bean)

Ivy Neck. Infrequent on sand bars and beaches at the Rhode River shore of Cheston Peninsula. Associate species: Cenchrus tribuloides, Spartina alterniflora. Higman IOI4.

S. umbellata (Muhl. ex Willd.) Britton

Pink Fuzzybean (S. umbellata (Muhl.) Britt.)

(Wild Bean)

Ivy Neck. One station: fallow field between entrance road and forested valley of Scaffold Creek drainage. Hectare 4668. Associate species: Desmodium perplexum, Echinochloa pungens, Eupatorium serotinum, Setaria lutescens. Higman iıi6.

\section{OXALIDACEAE}

Oxalis

I. Plant stemless, the leaves and scapes basal; petals purple or white. Expected (O. violacea).

I. Plant with leafy stem; petals yellow.

2. Petals I2-I8 mm long. Expected (O. grandis).

2. Petals 5-II mm long.

3. Stems prostrate, rooting at the node; stipules broad, brownish. Expected (O. corniculata).

3. Stems ascending, not rooting above the base; stipules narrow or wanting. 4. Fruiting pedicels straight, erect or ascending. Expected (O. europaea).

4. Fruiting pedicels deflexed, the capsules erect.

5. Lower internodes of flowering stems glabrous or strigose; styles I-2 mm long. 
6. Capsule densely puberulent, $15-25 \mathrm{~mm}$ long ......... O. stricta

6. Capsule glabrous or remotely pilose, $8-\mathrm{I} 2 \mathrm{~mm}$ long .... O. filipes

5. Lower internodes spreading pilose; styles $3-4 \mathrm{~mm}$ long .... O. florida

Oxalis stricta L.

Common Yellow Oxalis

(Wood Sorrel, Sheep Sorrel)

Java Farm. Infrequent to moderately abundant in old fields. Higman 760.

O. dillenii Jacq.

(O. filipes (combined with O. stricta

Slender Yellow Woodsorrel

in Britton and Brown))

Java Farm and Ivy Neck. Infrequent to moderately abundant in old fields; infrequent in open forest. Higman I7I, 390, 396, III5.

O. dillenii Jacq.

(O. florida Salisb. (combined with O. stricta

in Britton and Brown))

Slender Yellow Woodsorrel

(Wood Sorrel, Sheep Sorrel)

Java Farm. One station: side of ravine, of tributary to tidal portion of Muddy Creek. Hectare 3437. Canopy of Juglans nigra, Fagus grandifolia, Quercus alba, Quercus velutina. Higman $7 \mathrm{I} 8$.

\section{GERANIACEAE}

\section{Geranium}

I. Leaves $0.5^{-I .5} \mathrm{dm}$ wide, glabrous; rhizome at base of stem; petals much exceeding calyx; beak of mature style-column up to $2-3 \mathrm{~cm}$ long..... G. maculatum

I. Leaves not over $0.5 \mathrm{dm}$ wide, hirsute above, hirsute on nerves below; rhizome absent, taproots present; petals about equaling calyx; beak of mature stylecolumn $\mathrm{I}-2 \mathrm{~mm}$ long $\ldots \ldots \ldots \ldots \ldots \ldots \ldots . \ldots . \ldots . . . \ldots$. carolinianum

Geranium maculatum L.

Spotted Geranium

(Wild Geranium, Crane's-bill)

Java Farm. Infrequent; along old entrance road under mature canopy of Fagus grandifolia, Quercus alba. Higman 625.

G. carolinianum L.

Carolina Geranium

(Wild Geranium, Crane' s-bill)

Java Farm. One station in open, grassy triangle before entrance gate. Hectare 2463 . Associate species: Ranunculus bulbosus, Duchesnea indica, Fragaria virginiana, Cyperus strigosus. Higman 642 . 


\section{SIMAROUBACEAE}

Ailanthus

Ailanthus altissima (Mill.) Swingle

Tree of Heaven

Java Farm. One station in old field, approximate hectare 3508. Rhus radicans abundant. Higman I238.

\section{EUPHORBIACEAE}

I. Flowers with a calyx, not in a calyx-like involucre.

2. Stamens 2 or 3; styles simple. Expected (Phyllanthus).

2. Stamens 5 to many; styles divided.

3. Pubescence of branched or stellate hairs. Expected (Croton, Crotonopsis).

3. Pubescence of simple hairs or absent. (Also, flowers monoecious, the staminate ones very small, in spikes; stamens 8 to I6; pistillate flowers at the base of the spikes; styles 3, the stigmas cut-fringed, red) .... A Acalypha

I. Flowers without a calyx, in a calyx-like involucre. (Also, staminate flowers clustered around a single pistillate one; styles 3, each 2-cleft; outer structure (cyathium) surrounding the involucre and bearing 4 or 5 glands, these often with petaloid appendages) Euphorbia

Acalypha virginica L.

Acalypha

Virginia Threeseed Mercury

(Three-seeded Mercury)

Ivy Neck. Infrequent along trail to artificial pond, above source of Cheston Creek drainage. Associate species: Galinsoga ciliata, Gratiola neglecta, Ranunculus sceleratus, Perilla frutescens. Higman Io65.

\section{Euphorbia, Chamaesyce}

I. Leaves entire, symmetrical; appendages (on glands of cyathium) broadly obovate, petaloid; inflorescence terminal, umbellate . . . . . . . . . E. corollata

I. Leaves serrulate, oblique at base; appendages narrow or rudimentary; inflorescence of axillary clusters.

2. Stem semi-prostrate, villous; appendages white, narrow; ovary and capsule

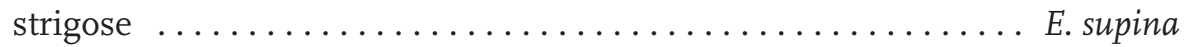

2. Stem erect or ascending, glabrous; appendages pink to maroon, very small; ovary and capsule glabrous (purple spot on leaves is not a consistent character $\ldots \ldots \ldots \ldots \ldots \ldots \ldots \ldots \ldots \ldots \ldots \ldots \ldots \ldots$ maculate

Euphorbia corollata L.

Flowering Spurge

Java Farm. Infrequent in old fields among dense Rubus sp., Rhus radicans, and Lonicera japonica. Higman $\mathrm{I} 37,388$. 
(not in Britton and Brown)

Java Farm. One station on Fox Point Road. Associate species: Digitaria ischaemum, Eragrostis spectabilis, Juncus tenius. Higman 28.

C. maculata (L.) Small

Spotted Sandmat

(E. maculata L.)

(Wortweed, Eyebane)

Java Farm. Infrequent in cracked pavement at main building area and along Fox Point and old entrance roads. Higman I4, I20, 543, 983.

\section{ANACARDIACEAE \\ Toxicodendron, Rhus}

I. Plant a woody vine, or with woody stems ascending I meter or higher; leaflets 3; flowers in axillary panicles; drupes whitish . . . . . . . . . . . .

I. Plant an erect woody shrub; leaflets II or more; flowers in terminal pyramidal panicles; drupes reddish.

2. Rachis of leaves winged; leaflets entire or nearly so . . . . . . . . copallina

2. Rachis of leaves not winged; leaflets coarsely serrate.

3. Branches, petioles, and drupes with long, dense pubescence... R. typhina

3. Branches and petioles glabrous, drupes minutely pubescent.... R. glabra

Toxicodendron radicans (L.) Kuntze ssp. radicans

Eastern Poison Ivy

(Rhus radicans L.)

(Poison Ivy)

Java Farm and Ivy Neck. Ubiquitous, but conspicuously infrequent under Quercus prinus canopy along estuary shores. Absent from salt marshes, but invading sparsely wooded sandbar at Sand Point, Cheston Peninsula.

T. radicans (L.) Kuntze ssp. radicans

Eastern Poison Ivy

( $R$. radicans, forma malanotrichocarpa (A. H. Moore) Fern.)

Java Farm. Old field near Fox Creek drainage. Dense, ascending woody stems 3 to 5 feet tall. Near southern limit of known range. Higman 375.

T. radicans (L.) Kuntze ssp. radicans

Eastern Poison Ivy (R. radicans, var. vulgaris (Michx.) DC., forma negundo (Greene) Fern.)

Java Farm. Twining on tree north of Fox Creek marsh. Near northern limit of known range. Higman 768 . 
Java Farm. One station in valley of Muddy Creek, upstream from culvert of old entrance road. Canopy of Fagus grandifolia, Quercus alba, Liriodendron tulipifera, etc. Hectare 2457.

Ivy Neck. Infrequent, in ditch through cornfields on Scaffold Peninsula, also on west shore of Scaffold Peninsula under canopy of Liquidambar styraciflua, Acer rubrum, etc. Higman I79, 85 I.

R. typhina L.

Staghorn Sumac

Java Farm. Infrequent, usually in scattered clumps; observed around main buildings, around burned house on road to springhouse (charcoal-covered soil), and along Rhode River shore near pier.

Ivy Neck. One clump near Cheston Point, and scattered nearby. Associate species: Prunus serotina, Robinia pseudoacacia. Infrequent in forest of central Scaffold Peninsula. Associate species there: Quercus alba, Liquidambar styraciflua, etc. Higman 222, 360 .

R. glabra L.

Smooth Sumac

Ivy Neck. Infrequent along top of bluff overlooking Rhode River, on Cheston Peninsula. Hectare 4768. Associate species: Maclura pomifera, Liquidambar styraciflua. Higman ıоII.

\section{AQUIFOLIACEAE}

Ilex

Ilex opaca Aiton

American Holly

(Ilex opaca Ait.)

Java Farm and Ivy Neck. Infrequent to moderately abundant along estuary shores, under canopy of Quercus prinus and Quercus alba. Occasional in mature upland forest or even in old fields. Higman 288, 522.

\section{CELASTRACEAE}

I. Plant a shrub, I-I.5 meters high; leaves opposite; capsule 5-valved, reddish-pink;

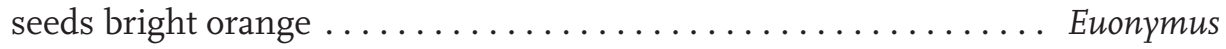

I. Plant a woody vine; leaves alternate; capsule 3-valved, yellow-orange; seeds red 
Java Farm. Infrequent, triangular area west of old Muddy Creek Road. Hectare 2472. Canopy of Fagus grandifolia and Liriodendron tulipifera. Higman 409.

Celastrus scandens L.

\section{Celastrus}

\section{American Bittersweet}

(Bittersweet)

Java Farm and Ivy Neck. One station each, both at top of bank overlooking Rhode River. Also found on an old field fence at Java Farm. Hectares 3407, 3600, 4890. Associate species at all sites: dense Rhus radicans. Higman 219, 496.

\section{ACERACEAE}

Acer

I. Leaves pinnately compound; young branches bright green; samaras forming a sharp, inverted $\mathrm{V} \ldots \ldots \ldots \ldots \ldots \ldots \ldots \ldots \ldots \ldots \ldots \ldots \ldots \ldots \ldots \ldots \ldots \ldots \ldots$ negundo

I. Leaves simple, palmately lobed; young branches not green; samaras forming a broad, inverted $\mathrm{U}$ or divergent.

2. Leaves 7-lobed, broader than long; sap milky; samaras widely divergent; leaves green beneath $\ldots \ldots \ldots \ldots \ldots \ldots \ldots \ldots \ldots \ldots \ldots \ldots \ldots$. platanoides

2. Leaves (3-) 5-lobed, usually longer than broad; sap clear; samaras forming an inverted $\mathrm{U}$ or moderately divergent; leaves white beneath.

3. Lobes extending more than halfway to midrib, sides of terminal and sometimes lateral lobes divergent; buds, flowers, petioles, young branches, and samaras yellowish-green ................... saccharinum

3. Lobes usually extending less than halfway to midrib, sides of all lobes converging; buds, flowers, petioles, young branches, and samaras (especially young) reddish to bright red ................ A. rubrum

Acer negundo L.

Boxelder

Java Farm and Ivy Neck. Infrequent in old fields and at margins of forest. Higman $427,577,620$.

A. platanoides L.

Norway Maple

Java Farm. One station: single seedling on trail along north boundary from old entrance gate to Muddy Creek. Hectare 247I. Canopy of Quercus alba, Liriodendron tulipifera, Acer rubrum, Fagus grandifolia, Carya tomentosa, etc. Higman Io78. 
Ivy Neck. One station: several large trees along marshy border of pasture (source of Cheston Creek). Hectare 4733. Grazing cows have prevented understory development and have deposited considerable manure. Canopy of Robinia pseudoacacia, Diospyros virginiana. Higman Io7I.

A. rubrum L.

Red Maple

Java Farm and Ivy Neck. Aggressive invader of abandoned fields, often co-dominant with Liquidambar styraciflua. Infrequent to moderately abundant in almost all forested areas, especially moister ones. Higman 4I7, 556.

\section{BALSAMINACEAE}

\section{Impatiens}

Impatiens capensis Meerb.

Jewelweed

(Impatiens capensis Meerb, f. immaculata (Weath.)

Fern. and Schub.)

(Touch-me-not)

Java Farm and Ivy Neck. Abundant in very moist, forested areas, especially the upper Muddy and Scaffold Creek valleys. Higman 76.

Impatiens capensis Meerb.

Jewelweed

(Impatiens capensis Meerb, f. albiflora (Rand and Redf.)

Fern. and Schub. (I. biflora Willd.)

(Touch-me-not)

Java Farm. One station: freshwater part of Fox Creek marsh. Hectare 3516. Higman 242.

\section{VITACEAE}

I Leaves simple, may be lobed; bark loosening in ropy shreds, without lenticels

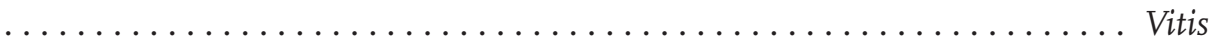

I. Leaves with 5 palmate leaflets; bark tight, with many lenticels . . . Parthenocissus

\section{Vitis}

I. Leaves ovate-squarish, with dense rusty pubescence beneath; grapes $\mathrm{I} .5-2.5 \mathrm{~cm}$ diameter ............................. labrusca

I. Leaves cordate at base, acuminate at tip, glabrous except for conspicuous tufts in axils of veins, beneath; grapes $3-9 \mathrm{~mm}$ diameter . . . . . . . . V vulpine

Vitis labrusca L.

Fox Grape

Java Farm. Infrequent in bottom of Muddy Creek valley. Canopy of Fraxinus americana. Associate species: Rubus sp., Lonicera japonica. Higman 67. 
Java Farm. Moderately abundant in old fields. Associate species: Rhus radicans, Campsis radicans, Lonicera japonica. Higman I66, 374.

\section{Parthenocissus}

Parthenocissus quinquefolia (L.) Planch.

(Parthenocissus quinquefolia L.)

Virginia Creeper

Java Farm. Infrequent in old fields and at borders of freshwater marshes. Associated with Rhus radicans, Lonicera japonica, etc. Higman 86, 224.

\section{MALVACEAE}

I. Salt marsh habitat; flowers white or pink-violet; carpels 5 .

2. Flowers 8-12 cm broad; petals white with red bases; leaves broadly ovate, 3-lobed, felt-pubescent . ..................... Hibiscus

2. Flowers 6-8 cm broad; petals roseate; leaves cordate-ovate; stellate-

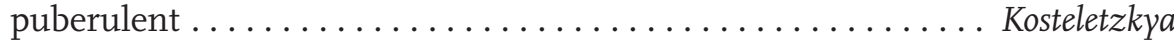

I. Roadside habitat; flowers yellow-orange; carpels $\mathrm{I} 2-\mathrm{I} 5 \ldots \ldots \ldots$. . . Abutilon

\section{Hibiscus}

Hibiscus moscheutos L.

(Hibiscus palustris L., forma peckii House)
Crimsoneyed Rosemallow

(Rose Mallow)

Java Farm and Ivy Neck. Moderately abundant at shoreward margins of wet salt marshes and marshy estuaries. Associate species: Typha latifolia, T. angustifolia, Kosteletzkya virginica, Iva frutescens, Baccharis halimifolia, Panicum virgatum, etc. Grows just seaward of the Smilax rotundifolia zone. Higman 78.

\section{Kosteletzkya}

Kosteletzkya virginica (L.) C. Presl ex A. Gray (Kosteletzkya virginica (L.) Presl.)

Virginia Saltmarsh Mallow (Seashore Mallow)

Java Farm and Ivy Neck. Moderately abundant in same habitat as Hibiscus palustris above. Higman 57, 28I.

Abutilon

\section{Abutilon theophrasti Medik.}

Velvetleaf

(Abutilon theophrasti Medic.)

(Velvet-leaf)

Ivy Neck. One station: border of cornfield beside old barn south of artificial pond, near source of Cheston Creek. Hectare 4723. Associate species: Datura stramonium, Plantago major, Eleusine indica, and Polygonum persicaria. Higman Iooo. 


\section{CLUSIACEAE \\ (GUTTIFERAE)}

I. Sepals 4 , in 2 unequal pairs; petals $4 \ldots \ldots \ldots \ldots \ldots \ldots$. . . . . . . . . . . . .

I. Sepals 5 ; petals $5 \ldots \ldots \ldots \ldots \ldots \ldots \ldots \ldots \ldots \ldots \ldots \ldots \ldots$ Hypericum

\section{Hypericum}

(Ascyrum)

Hypericum hypericoides (L.) Crantz ssp. hypericoides (Ascyrum hypericoides L.)

Ivy Neck. One station: abandoned road near southern border of central mixed hardwood forest, Scaffold Peninsula. Canopy of Quercus alba, Quercus velutina, Fagus grandifolia, etc. Higman 968.

\section{Hypericum}

I. Flowers flesh-color or mauve, clustered in axils and top of stem. Expected (H. virginicum).

I. Flowers yellow, in terminal cymes.

2. Stamens 20-100 or more.

3. Stamens in 3 to 5 clusters; styles 3; capsules, petals, sepals, and leaves with black dots or lines (oil vesicles), leaf dots may also be translucent.

4. Plant very branched; leaves very numerous, elliptic-linear, usually with axillary fascicles on whole stem and branches; petals with black marginal dots; sepals $5-6 \mathrm{~mm}$ long, acuminate........... H. perforatum

4. Plant branched only at summit for cyme; leaves remote, elliptic-ovate, seldom with axillary fascicles except on upper stem near cyme; petals with black lines; sepals $2-3 \mathrm{~mm}$ long, acute or blunt .... H. punctatum

3. Stamens not in clusters; styles united into a sharp beak; capsules, petals, sepals, and leaves without oil vesicles or black markings. Expected (H. densiflorum).

2. Stamens 5 to I2, if up to 20 the flowers are very small.

5. Leaves spreading, not linear-subulate.

6. Leaves ovate-deltoid or round-oblong, the upper cordate or clasping at base ............................... Hutilum

6. Leaves lanceolate or linear to narrowly obovate, tapering to sessile or short-petioled base. Expected (H. canadense).

5. Leaves appressed or strongly ascending, linear-subulate. Expected (H. gentianoides). 
Java Farm. One station: western part of main building area. Hectare 2499. Associate species: Erigeron canadensis, Achillea millefolium, Setaria faberii, Plantago lanceolata.

Ivy Neck. Moderately abundant in abandoned wheatfield, especially near road. Higman 7, 8I2, 955, 956.

H. punctatum Lam.

Spotted St. Johnswort

(St. John's-wort)

Ivy Neck. Infrequent along abandoned road through forest of Scaffold Peninsula. Canopy of Quercus alba, Liriodendron tulipifera, Liquidambar styraciflua, Carya tomentosa, Nyssa sylvatica. Higman 928, 929.

H. mutilum L.

Dwarf St. Johnswort

(St. John's-wort)

Java Farm. One station: border of Phalaris arundinacea meadow, north of main building area. Hectare 2560. Higman ${ }_{5} 6$.

\section{CISTACEAE}

Lechea

Lechea racemulosa Michx.

Illinois pinweed

(Pinweed)

Ivy Neck. One station: south shore of Scaffold Peninsula. Hectare 5763. Canopy of Quercus prinus. Higman I049.

\section{VIOLACEAE}

Viola

I. Stemless; petioles and peduncles rising from thick rhizome; flowers blue to violet.

2. Sepals ciliate.

3. Petioles and both or only the lower leaf surfaces villous .......V. sororia

3. Petioles glabrous; only upper leaf surface villous or strigose ......... $\ldots \ldots \ldots \ldots \ldots \ldots \ldots \ldots \ldots \ldots \ldots \ldots \ldots \ldots \ldots \ldots \ldots \ldots$ septentrionalis

2. Sepals glabrous (rest of plant same except bearded lateral petals) . . . . . .

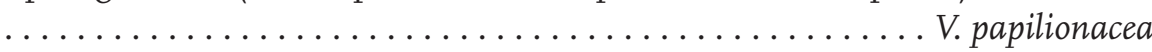

I. Stem leafy, rising from taproot; flowers white to bluish $\ldots \ldots \ldots \ldots \ldots \ldots$ V. kitaibeliana, var. rafinesquii 
Ivy Neck. Abandoned road through central Scaffold Peninsula. Canopy of Quercus alba, Quercus velutina, Nyssa sylvatica, Liriodendron tulipifera, Carya tomentosa. Higman 599.

V. septentrionalis Greene

Northern Woodland Violet

(V. septentrionalis Green.)

(Violet)

Java Farm. Infrequent along old Muddy Creek Road. Canopy of Fagus grandifolia, Quercus alba. Higman 548.

V. sororia Willd.

Common Blue Violet

(V. papilionacea Pursh.)

(Meadow Violet)

Java Farm. Moderately abundant on steep east-facing slope adjacent north border. Hectare 2573. Canopy of Juglans nigra. Higman 58I, 602, 629, 630.

\section{V. bicolor Pursh}

Field Pansy

(V. kitaibeliana R. and S., var. rafinesquii Fern.)

(V. rafinesquii Greene))

(Wild Pansy)

Java Farm. Infrequent on south-facing slope adjacent north border, overlooking meadow. Hectare 2550. Open canopy of Liriodendron tulipifera, Pyrus malus, Robinia pseudoacacia. Found in scattered grassy areas. Higman II5O.

(NOTE: All violet species except the last appear to be moderately abundant throughout the more moist forested areas, especially on Java Farm. No clear pattern of distribution has yet been determined.)

\section{PASSIFLORACEAE}

Passiflora

Passiflora lutea L.

Yellow Passionflower

(Passion-flower)

Ivy Neck. One station: west side of southern Cheston Peninsula. Low bluff overlooking big salt marsh. Hectare 5749. Canopy of Quercus velutina and Carya tomentosa. Higman I075. 


\section{LYTHRACEAE}

I. Flowers regular or nearly so. 2. Calyx short, campanulate to globular. Expected (Rotala, Ammannia).

2. Calyx tubular. (Also, petals white; leaves linear) .......... Lythrum

I. Flowers irregular, the tubular calyx spurred at base on the upper side. (Also,

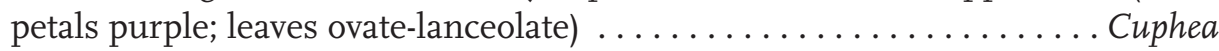

$\begin{array}{rr}\text { Lythrum lineare L. } & \text { Whthrum } \\ \text { (Loosestrife) }\end{array}$

$$
\text { Lythrum }
$$

Java Farm. One station: Fox Creek salt marsh. Hectare 3527. Iva frutescens and Distichlis spicata dominant. Higman 6r.

Cuphea viscosissima Jacq.

\section{Cuphea}

(Cuphea petiolata (L.) Koehne)

Blue Waxweed

Ivy Neck. One station: moist woods along Cheston Creek drainage. Hectare 4752. Canopy of Platanus occidentalis, Diospyros virginiana, and Liquidambar styraciflua. Associate species: Duchesnea indica, Eupatorium coelestinum, Ranunculus sceleratus, Viola sp. Higman Ioo5.

\section{CORNACEAE}

(NYSSACEAE)

$$
\text { Nyssa }
$$

Nyssa sylvatica Marshall

(Nyssa sylvatica Marsh.)

Blackgum

(Black Gum, Sour Gum)

Java Farm and Ivy Neck. Infrequent to moderately abundant in a variety of mixed hardwood canopies, especially oaks. Higman I09, 42I, 520.

\section{ONAGRACEAE}

I. Flowers 2-merous; fruit indehiscent, bristly $\ldots \ldots \ldots \ldots \ldots \ldots$ Circaea

I. Flowers 4-merous (5-or 6-merous in Jussiaea); fruit dehiscent.

2. Fruit indehiscent, with I to 4 seeds. Expected (Gaura).

2. Fruit a dehiscent capsule (loculicidal), many-seeded.

3. Leaves alternate; petals showy; capsule stoutly cylindric ....... Oenothera

3. Leaves opposite; petals inconspicuous or absent; capsule linear-cylindric or short and angled to terete. 
4. Stamens 4; capsule short; seeds glabrous........... Ludwigia

4. Stamens 8; capsule linear-cylindric, its valves widely splitting; seeds with apical tufts of hair, like the bristle pappus on achenes of Compositae ...................................... Epilobium

\author{
Circaea \\ Circaea lutetiana L. ssp. canadensis (L.) \\ Asch. \& Magnus \\ Broadleaf Enchanter's Nightshade \\ (Enchanter's Nightshade) \\ (Circaea quadrisulcata (Maxim.) Franch. and Sav., \\ var. canadensis (L.) Hara)
}

Java Farm. One station: along old entrance road between inter-sections with Fox Point road and springhouse road. Hectare 3409.

Ivy Neck. One station: moist woods at Cheston Creek drainage. Hectare 4752. Canopy of Diospyros virginiana, Platanus occidentalis, Liquidambar styraciflua. Associate species: Eupatorium coelestinum, Duchesnea indica, Cuphea petiolata, Ranunculus sceleratus. Higman 821, 855 .

\title{
Oenothera
}

I. Leaves lyrate-pinnatifid; stem leafy to base, often branching at base. (Also, cap-

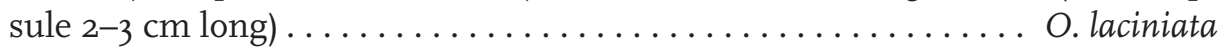

I. Leaves entire or shallowly serrate; stem often nearly leafless below the middle, branching above the middle.

2. Calyx tube $\mathrm{I} .8-4.4 \mathrm{~cm}$ long; capsules $\mathrm{I}-3.5 \mathrm{~cm}$ long, lance-cylindric, nearly sessile, appressed ascending ...................... biennis

2. Calyx tube .5-I.5 $\mathrm{cm}$ long; capsules 6-II mm long, short-ellipsoid, tapering to slender stipes $5-15 \mathrm{~mm}$ long, ascending-spreading $\ldots \ldots \ldots \ldots$. fruticose

Oenothera laciniata Hill

Cutleaf Evening Primrose (Evening Primrose)

Ivy Neck. One station in abandoned field on Scaffold Peninsula. Hectare 476I. Associate species: Hypericum perforatum, Trifolium pratense, Trifolium hybridum, Eupatorium serotinum, Plantago virginica. Higman 690.

O. biennis L.

Common Evening Primrose (Evening Primrose)

Java Farm. One station on Fox Point Road.

Ivy Neck. Infrequent in grassy abandoned field on Scaffold Peninsula. Associated with O. laciniata above. Higman I45, 954, IOI8. 
Ivy Neck. Moderately abundant in abandoned field on Scaffold Peninsula. Associated with $O$. laciniata and O. biennis and their associates above. Higman III4.

\section{Ludwigia}

I. Leaves alternate; stems erect; flowers pedicelled; petals yellow, conspicuous $\ldots \ldots \ldots \ldots \ldots \ldots \ldots \ldots \ldots \ldots \ldots \ldots \ldots \ldots \ldots \ldots \ldots \ldots \ldots \ldots \ldots \ldots \ldots$ alternifolia

I. Leaves opposite; stems ascending to depressed; flowers sessile; petals purple to

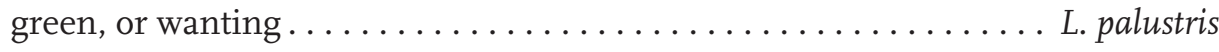

Ludwigia alternifolia L.

Seedbox

Ivy Neck. One station: marsh at head of Cheston Creek estuary. Hectare 4753. Associate species: Typha latifolia, Scirpus americana, Panicum clandestinum, Eleocharis intermedia. Higman ııо.

L. palustris (L.) Elliott

Marsh Seedbox

(L. palustris (L.) Ell., var. americana (DC.) Fern. and Grisc.)

Ivy Neck. Infrequent on muddy shore of artificial pond near source of Cheston Creek drainage. Hectare 4723. Associate species: Eleocharis sp., Echinochloa pungens, Limnobium spongia, Gratiola neglecta, Juncus acuminatus, Ranunculus sceleratus. Higman 995.

Epilobium coloratum Biehler

\section{Epilobium}

Purpleleaf Willowherb

(Willow-herb)

Java Farm. Two stations: old field on north side of main buildings, dominated by woody vines. Hectare 2580. Also in deep ditch of Fox Creek drainage. Hectare 2583. May be infrequent elsewhere. Higman I95, 327.

Myriophyllum spicatum L.

HALORAGACEAE

(HALORAGIDACEAE)

Myriophyllum

Eurasian Watermilfoil

(Eurasian Water Milfoil)

Java Farm and Ivy Neck. Abundant in estuaries, especially Muddy Creek estuary. Found along Muddy Creek to upstream limit of salt marsh vegetation. (Virtually disappeared in 1967$)$. 


\section{ARALIACEAE}

I. Leaves 2-or 3-times compound; plant a thorny shrub (in the species collected) ......................................... Aralia

I. Leaves simple, palmately lobed; plant a thornless woody vine........ Hedera

$\begin{array}{rr}\text { Aralia spinosa L. } & \text { Aralia } \\ & \text { Devil's Walkingstick } \\ \text { (Hercules' Club) }\end{array}$

Java Farm. Principal station on north side of Fox Point Road, near base of peninsula and head of Fox Creek estuary. Hectare 3556. Abundant in understory. Canopy of Quercus velutina, Quercus alba, Nyssa sylvatica, and Pinus virginiana. Also observed in Hectares 3415, 3530, and 3554. Scattered infrequently under a variety of hardwood canopies.

Ivy Neck. Infrequent in forest on lower Scaffold Peninsula. Canopy of mixed hardwoods. Higman 395.

Hedera helix L.

\section{Hedera}

Java Farm. Principal station north of Smithsonian property, on north-facing slope above stream, near road to pier. Hectare 2599. Canopy of mixed hardwoods; understory of Ilex opaca. Also on north bank of Fox Point. Hectare 3558. Canopy of Quercus prinus. Higman I239.

\section{APIACEAE \\ (UMBELLIFERAE)}

(From Dayton, 1942)

I. Leaves without blades, reduced to hollow, linear, cylindrical, septate petioles. Expected (Lilaeopsis).

I. Leaves with blades well-developed.

2. Leaves simple (only the basal ones in Zizia).

3. Stems creeping, rooting at the nodes ................ Hydrocotyle

3. Stems erect. Expected (Eryngium, Zizia).

2. Leaves compound.

4. Leaves, or most of them, palmately compound.

5. Umbellets globose; fruit burlike, with hooked bristles ....... Sanicula

5. Umbellets not globose; fruit not burlike nor bristly.

6. Umbels conspicuously irregular; flowers white; calyx teeth and involucels absent; fruit narrowly oblong, bony, often curved ......... 
6. Umbels regular; flowers yellow; calyx teeth and involucels prominent; fruit oblong or oval. Expected (Zizia).

4. Leaves not palmately compound.

7. Leaves ternately compound or decompound. Expected (Zizia, Chaerophyllum, Osmorhiza).

7. Leaves pinnate or pinnately decompound.

8. Plants annual.

9. Plants glabrous; leaf segments filiform; fruits glabrous; involucral bracts pinnately parted .................. Ptilimnium

9. Plants rough-pubescent; leaf segments lanceolate; fruits with hooked bristles; involucral bracts small, lanceolate, sometimes absent. Expected (Torilis).

8. Plants biennial or perennial.

Io. Plant pubescent.

II. Plant bristly-hispid; involucre conspicuous, of leafy, pinnately parted bracts; fruit oblong, barbed-bristly on the winged ribs ....................... Daucus

II. Plant closely pilose above; involucre absent; fruit oval or ovate, pubescent (not barbed), winged laterally, flattened dorsally. Expected (Angelica).

Io. Plant glabrous.

I2. Flowers yellow; plants mesophytic, in fields, etc. Expected (Pastinaca).

I2. Flowers white; plants hydrophytic, poisonous.

I3. Fruit wavy-ribbed; involucre and involucels of ovateacuminate bracts; calyx teeth absent. Expected (Conium).

I3. Fruit with straight ribs or winged; involucre and involucels of narrow bracts or the former absent; calyx teeth present (may be minute).

I4. Fruit winged laterally, flattened dorsally. (Also, roots tuberous; leaves simply pinnate; leaflets entire or remotely toothed; calyx teeth evident). Expected (Oxppolis).

I4. Fruit not winged laterally. (Also, umbels compound; fruit ovate to oblong, or orbicular in Cicuta).

I5. Leaves simply pinnate; stems corrugated; involucre present; fruit with prominent corky ribs and broad red-brown furrows; calyx teeth minute $. . . \ldots \ldots \ldots \ldots . . \ldots$. Sium 
Hydrocotyle verticillata Thunb.

I5. Leaves twice or thrice pinnate; stems terete; involucre usually absent; fruit with rounded corky ribs and dark furrows; calyx teeth prominent. (Also, leaf veins tend in direction of notches between teeth). Expected (Cicuta).

\section{Hydrocotyle}

(Hydrocotyle verticillata Thunb.)

Whorled Marshpennywort

(Collected by H. David Hammond)

(Water Pennywort)

Java Farm. Probably along Fox Point Road.

Sanicula

Sanicula canadensis L.

Canadian Blacksnakeroot

(Black Snakeroot)

Java Farm. One station: new entrance road, near culvert of Fox Creek drainage. Hectare 2573. Canopy of Juglans nigra and Liquidambar styraciflua. Higman 69, 3I0.

Cryptotaenia canadensis (L.) DC. $\quad \begin{array}{r}\text { Canadian Honewort } \\ \text { (Honewort, Wild Chervil) }\end{array}$

Cryptotaenia

(Honewort, Wild Chervil)

Java Farm. Moderately abundant along old entrance road, from entrance gate nearly to main building area. Canopy of Quercus alba, Fagus grandifolia, etc. over most stations. Higman 793, 820, 933, 980.

Ptilimnium

Ptilimnium capillaceum (Michx.) Raf.

Herbwilliam

(Mock Bishop-weed)

Java Farm. One station: shore of Muddy Creek near upstream limit of salt marsh. Hectare 3403. Associate species: Panicum virgatum, Baccharis halimifolia, Aster subulatus, Cyperus strigosus. Higman 254.

\section{Daucus}

Daucus carota L.

Queen Anne's Lace (Wild Carrot)

Java Farm and Ivy Neck. Infrequent; in grassy old fields, along roadsides, etc. Higman 138, 199, 953 . 
Ivy Neck. One station: margin of cut-off pond on Rhode River shore of Cheston Peninsula. Hectare 5300. Associate species: Sambucus canadensis, Rumex verticillatus, Sparganium eurycarpum. Higman 948.

\section{CORNACEAE}

I. Flowers green or purple, in a close cyme or head, with a large 4-bracted white or pink involucre; fruit red $\ldots \ldots \ldots \ldots \ldots \ldots \ldots \ldots \ldots \ldots \ldots \ldots \ldots \ldots$ florida

I. Flowers white or creamy, in open cymes, without involucre; fruit blue or white.

2. Leaves alternate, but appearing whorled by crowding at tips of branches ...

2. Leaves opposite, not crowded at tips of branches.

3. Cymes long, loosely paniculate; leaves I to $4 \mathrm{~cm}$ broad. (Also, pedicels bright red). Expected (C. racemosa).

3. Cymes compact, broad and flat-topped; leaves I. 5 to I3 $\mathrm{cm}$ broad.

4. Pith of branches I to 2 years old white. Expected (3 species).

4. Pith of branches I to 2 years old brown. (Also, leaves smooth above, appressed puberulent beneath; fruit blue) ............ . amomum

\section{Cornus}

Cornus florida L.

Flowering Dogwood

Java Farm and Ivy Neck. Infrequent to moderately abundant in abandoned fields; associate species: Liquidambar styraciflua, Acer rubrum, Quercus palustris. Also infrequent to moderately abundant in mature upland forest of old entrance road, under canopy of Quercus alba, Fagus grandifolia, Liriodendron tulipifera, etc. Higman 33, 603.

C. alternifolia L. f.

Alternateleaf Dogwood

(Green Osier, Pagoda-dogwood)

Java Farm. One station: west slope of Hog Island. Canopy of Quercus alba and Quercus prinus. Associate species: Kalmia latifolia, Viburnum acerifolium. Hectare 3592. Higman I03.

C. amomum Mill.

Silky Dogwood

(Red Willow)

Java Farm. Infrequent in valley of Muddy Creek under canopy of Fraxinus americana, and in freshwater marsh at western boundary, west of old Muddy Creek Road. 
Ivy Neck. One station near source of a tributary to the east fork of Scaffold Creek estuary. Hectare 4677. Associate species: Acer rubrum, Liquidambar styraciflua. Higman 7I, I86, 4I3.

\section{PYROLACEAE, MONOTROPACEAE}

I. Leaves green, well-developed; plant self-supporting.

2. Stem leafy; flowers in a corymb. (Also, in species collected, leaves thick and shiny, with white veins) $\ldots \ldots \ldots \ldots \ldots \ldots$ Chimaphila

2. Stem scapose, leafy only near base; flowers in a raceme. Expected (Pyrola).

I. Leaves white, scaly; plant saprophytic or root-parasitic.

3. Plant white, fleshy, odorless; flowers I or few, nodding; petals 4 to 6 , separate; calyx of 2 to 5 bracts; deciduous forest habitat ................

3. Plant purple-brown, somewhat fleshy, sweet-scented when fresh; flowers several in a raceme, nodding at first; petals united; calyx of 5 regular sepals; pine forest habitat. Expected (Monotropsis).

\section{Chimaphila maculata (L.) Pursh}

\section{Chimaphila}

(Chimaphila maculata (L.) Pursh.)

Striped Prince's Pine

(Wintergreen, Waxflower)

Java Farm. Infrequent to moderately abundant on forested slopes overlooking Muddy Creek valley and Fox Creek estuary. Canopy usually of Quercus alba, Acer rubrum, Liquidambar styraciflua, and a few Pinus virginiana. Higman 94, 884.

Monotropa uniflora L.

Monotropa

Indianpipe
(Indian-pipe, Corpse-plant)

Java Farm. One station: among leaf litter at base of dead Quercus alba, along north boundary between old entrance gate and Muddy Creek. Higman iı82.

\section{ERICACEAE}

I. Ovary inferior; fruit a dark blue to black berry. (Also, corolla 5-cleft; stamens Io). 2. Leaves with many amber glandular dots; ovary ıo-locular; anthers not awned

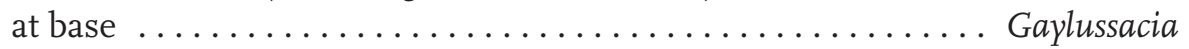

2. Leaves not glandular-dotted; ovary either 5 locular and the anthers awnless, or ovary Io-locular and anthers awned at base .......... Vaccinium

I. Ovary superior; fruit a capsule, berry-like in Gaultheria.

3. Plant prostrate and trailing, sub-herbaceous.

4. Leaves acute at base, glabrous or sparsely bristly beneath; fruit berry-like, red. Expected (Gaultheria). 
4. Leaves round to cordate at base, red-hirsute beneath; fruit a capsule. (Also, stems, petioles, and capsules hirsute) $\ldots \ldots \ldots \ldots \ldots \ldots$ Epigaea

3. Plant an erect shrub.

5. Corolla globose, ovoid, or tubular; capsule loculicidal. (Also, anthers open at apex; flowers white, in lateral racemes or umbelliform clusters). Expected (Lyonia, Leucothoe).

5. Corolla funnelform, campanulate, or saucer-shaped; capsule septicidal.

6. Corolla funnelform, irregularly lobed; capsule cylindric, IO-25 $\mathrm{mm}$ long. (Also, flowers pink, leaves deciduous in species collected)... Rhododendron 6. Corolla saucer-shaped, regular; capsule depressed-globose, less than

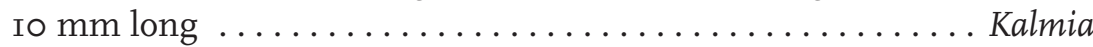

\section{Gaylussacia}

I. Leaves copiously glandular-dotted on both sides; fruit black, not glaucous .... ............................................ baccata

I. Leaves moderately glandular-dotted, on underside only; fruit dark blue, glaucous ................................... G. frondosa

Gaylussacia baccata (Wangenh.) K. Koch

Black Huckleberry (Gaylussacia baccata (Wang.) K. Koch)

Java Farm and Ivy Neck. Moderately abundant under canopy of Quercus prinus, along banks of estuaries and on Hog Island. Also at Java Farm, infrequent on slopes overlooking Muddy Creek valley (hectares 2467 and 34I5) under canopy of Fagus grandifolia and Quercus alba. Associate species: Kalmia latifolia, Rhododendron nudiflorum, Vaccinium staminium. Higman Io6, IIо, 475, 675.

G. frondosa (L.) Torr. \& A. Gray ex Torr.

(G. frondosa (L.) T. and B.)

Blue Huckleberry

(Dangleberry)

Java Farm. Infrequent on Hog Island (hectare 3592) under Quercus prinus canopy. Associated with G. baccata and its associate species, above. Higman III.

\section{Vaccinium}

I. Leaves not more than I cm long, rounded at both ends; stem trailing; corolla cleft to below the middle, its lobes recurved. Expected (V. macrocarpon).

I. Leaves 2-7 cm long, acute at both ends (or some apically rounded in V. vacillans); stem erect; corolla shallowly cleft.

2. Flowers subtended by leafy bracts much smaller than the foliage leaves, in panicles or racemes; anthers 2-awned on the back. (Also, leaf veins thinly puberulent on both sides; corolla white .............. V. stamineum

2. Flowers not subtended by bracts, in small racemes or corymbs; anthers awnless.

3. Leaves finely and sharply serrulate (also entire in V. corymbosum). 
4. Leaves narrowly elliptic, 2-3 cm long, not over I $\mathrm{cm}$ wide. Expected (V. angustifolium).

4. Leaves broadly elliptic or ovate, $4-6 \mathrm{~cm}$ long, $2 \mathrm{~cm}$ wide or more. (Also, corollas pink; berries blue to black, glaucous) . . . . . . V. corymbosum

3. Leaves entire, occasionally slightly serrulate near the apex. (Also, corolla usually pink to reddish, cylindric to urceolate; berry blue to black, 5-8 mm dia.).

5. Leaves elliptic to oval, the smaller lateral ones more circular; twigs often bright green. (Also, berry dark blue, faintly glaucous) ..... V. vacillans

5. Leaves all elliptic or ovate, acute; twigs dull green or brownish.

6 . Leaves very tomentulose beneath, especially on the veins; berries shiny black, not glaucous.

6. Leaves sparsely puberulent or glabrous beneath; berries dark blue to blue-black, glaucous.

7. Leaves 3-5 cm long; corolla 5-6 $\mathrm{mm}$ long, dull white; berry 5-8 $\mathrm{mm}$ dia. Expected (V. caesariense)

7. Leaves 5-8 mm long; corolla 7-I2 $\mathrm{mm}$ long, bright white; berry 6-I2 mm dia. ....................... corymbosum

Vaccinium stamineum L.

Deerberry

Java Farm. Infrequent along old Muddy Creek Road and on upland slopes of Muddy Creek valley. Canopy of Fagus grandifolia, Quercus alba, Quercus prinus. Associate species: Gaylussacia baccata. Higman 647, 67I.

\section{V. pallidum Aiton}

Blue Ridge Blueberry

(V. vacillans Torr.)

(Low Bilberry)

Java Farm. Moderately abundant on Fox Point peninsula. Hectare 3559. Canopy of Quercus prinus. Associate species: Rhododendron nudiflorum, Vaccinium stamineum, Gaylussacia baccata, Kalmia latifolia. Higman I240.

V. corymbosum L.

Highbush Blueberry (High-bush Bilberry)

Ivy Neck. One station: southern shore of Scaffold Peninsula. Hectare 5763. Canopy of Quercus prinus, Quercus alba, Carya tomentosa, and Fagus grandifolia. Higman 845.

Epigaea repens L.

$$
\text { Epigaea }
$$

Trailing Arbutus

(Mayflower)

Java Farm. Infrequent at Fox Point and Hog Island. Hectares 3559 and 3592. Canopy of Quercus prinus. Associate species: Gaylussacia baccata, Kalmia latifolia, Vaccinium staminium. Higman 105, 567. 


\section{Rhododendron}

I. Flowers preceding the leaves or accompanying the young ones.

2. Plants colonial, with subterranean stolons; corolla buds with rows of stalked glands; corolla glabrous within. Expected ( $R$. atlanticum).

2. Plants not strikingly colonial; corolla buds glandless; corolla tube pubescent within.

3. Leaves pubescent beneath; flowers fragrant. Expected (R. maximum, R. atlanticum).

3. Leaves glabrous beneath or with strigose midrib; flowers not fragrant. (Also, pedicels and calyx not glandular; corolla tube pilose without) . . . . . $\ldots \ldots \ldots \ldots \ldots \ldots \ldots \ldots \ldots \ldots \ldots \ldots \ldots \ldots \ldots \ldots \ldots \ldots$ nudiflorum

I. Flowers expanding after the leaves.

4. Young branchlets and leaves glabrous; calyx lobes not glandular; ovary densely glandular-villous. Expected (R. arborescens).

4. Young branchlets bristly or strigose; leaves bristly on the midrib beneath; pedicels and calyx lobes glandular-hirsute; ovary strigose, not glandular . . . . .................................... viscosum

Rhododendron periclymenoides (Michx.) Shinners

Pink Azalea (Rhododendron nudiflorum (L.) Torr.)

(Election-pink, Pinxter-flower)

Java Farm. Infrequent on Fox Point (hectare 3559; canopy of Quercus prinus) and on west-facing slope overlooking Muddy Creek (hectare 34I5; canopy of Fagus grandifolia and associates). Canopy at both sites includes Quercus alba, Q. velutina, and Carya tomentosa. Associate species at both stations: Gaylussacia baccata, Kalmia latifolia, Vaccinium spp. Higman IO2, 4 OI.

R. viscosum (L.) Torr.

Swamp Azalea

(Swamp Honeysuckle)

Java Farm. Infrequent at Fox Point. Hectare 3559. Canopy and associates as for R. nudiflorum, above. Higman 646 .

Kalmia latifolia L.

Kalmia

Java Farm. Infrequent to moderately abundant on moderately steep, usually westfacing slopes overlooking Muddy Creek and its tributaries. Canopy of Fagus grandifolia, Quercus alba, Liriodendron tulipifera.

Dominates understory on steep west-facing slope of Hog Island, under canopy of Quercus alba and Quercus prinus. Less frequent under the same canopy at Fox Point and along estuary shoreline at Ivy Neck. Associate species: Gaylussacia baccata. Higman 8 oI. 


\section{PRIMULACEAE}

Anagallis

Anagallis arvensis L.

Scarlet Pimpernel

Ivy Neck. One station: beside tractor trail to abandoned field on Scaffold Peninsula. Hectare 4740. Associate species: Galinsoga ciliate, Mollugo verticillata, Diodia teres, Polygonum aviculare. Higman 835.

\section{PLUMBAGINACEAE}

Limonium

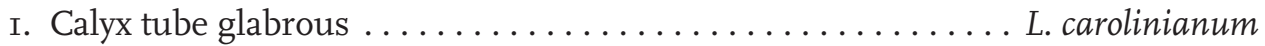

I. Calyx tube pubescent at base and part way up the angled sides ....... L. nashi

Limonium carolinianum (Walter) Britton

Lavender Thrift

(Limonium carolinianum (Walt.) Britt.)

(Sea Lavender)

Java Farm and Ivy Neck. Infrequent in flooded parts of salt marshes. Associate species: Spartina alterniflora, Spartina cynosuroides, Baccharis halimifolia. Higman 993.

L. carolinianum (Walter) Britton

(L. nashii Small)

Lavender Thrift

(Small Sea Lavender)

Java Farm and Ivy Neck. Infrequent in flooded parts of salt marshes. Associated with L. carolinianum above. Higman 2I2.

\section{EBENACEAE}

Diospyros virginiana L.

Diospyros

Common Persimmon

(Persimmon)

Java Farm. Infrequent in old fields. Associate species: Quercus palustris, Acer rubrum, Liquidambar styraciflua.

Ivy Neck. Infrequent in old fields as above. Also moderately abundant in canopy along drainage of Cheston Creek. Associate species there: Robinia pseudoacacia, Platanus occidentalis, Liquidambar styraciflua.

Java Farm and Ivy Neck. Infrequent in salt marshes, apparently the less saline parts. Associate species: Panicum virgatum, Typha latifolia. Also on sandbars. Higman II6, I58, 233, 293, 383, 512, 746. 


\section{OLEACEAE}

I. Plant a large tree; leaves pinnate; fruit a samara $\ldots \ldots \ldots \ldots \ldots$. . . . Fraxinus

I. Plant a woody shrub or small tree; leaves simple; fruit a wingless capsule, drupe or berry.

2. Fruit a loculicidal capsule. (Also, plant cultivated or escaped; flowers lilacpurple; leaves ovate, acuminate, entire, petioled, truncate or subcordate at

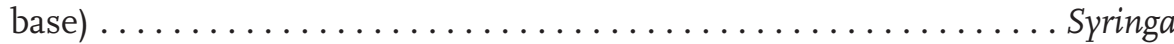

2. Fruit a drupe or berry.

3. Petals linear, nearly separate. Expected (Chionanthus).

3. Petals united, funnelform corolla with broad lobes. (Also, panicles terminal, erect; stigma 2-lobed; berry 2-locular) . . . . . . . . Ligustrum

\section{Fraxinus}

I. Leaflets definitely on wingless petiolules. (Also, leaflets acuminate, with round or broadly acute base; samaras with a distinct flat wing and terete body).

2. Leaflets abruptly acuminate, equilateral at base, oblong to ovate or obovate; samaras $3-5 \mathrm{~cm}$ long, the wing $4-7 \mathrm{~mm}$ broad, the body $0.7-\mathrm{I} .6 \mathrm{~cm}$ long. (Also, leaflets may be serrulate near summit)........... F. americana

(Typical species has glabrous branchlets, petioles, and lower surfaces of leaflets; var. biltmoreana has these pubescent and the leaflets white beneath).

2. Leaflets long-acuminate, inequilateral at base, lanceolate to oblong or elliptic; samaras (4) $5.5(7.5) \mathrm{cm}$ long, the wing 6-I2 $\mathrm{mm}$ broad, the body I-3 $\mathrm{cm}$ long. (Also, branchlets, petioles, and leaf rachiese velvet-tomentose) . . . F. tomentosa

I. Leaflets sessile, or subsessile on winged petiolules. (Also, leaflets lanceolate to oblong, with broadly acute base).

3. Leaflets entire or undulate, rarely serrulate, often inequilateral at base, on winged petiolules; samaras $4-7.5 \mathrm{~cm}$ long, wing extending to middle of body. (Also, leaflets usually 7).

(Typical species has pubescent branchlets, petioles, panicles, and lower surfaces of leaflets; var. subintegerrima has these glabrous) $\ldots \ldots \ldots \ldots$. . . . . . . . . . . . . . . . . . . . . . . .

$\ldots \ldots \ldots \ldots \ldots \ldots \ldots \ldots \ldots \ldots \ldots \ldots \ldots \ldots \ldots \ldots \ldots \ldots \ldots \ldots \ldots$. pennslvanica

4. Leaflets conspicuously serrate, equilateral at base, sessile; samaras 2.5-4 $\mathrm{cm}$ long, 6-Io $\mathrm{mm}$ broad, the body poorly defined, wing extending to base. Expected (F. nigra).

Fraxinus americana $\mathrm{L}$.

White Ash

(Fraxinus americana L., var. biltmoreana (Beadle) J. Wright)

Java Farm. Canopy dominant along bottom of Muddy Creek valley above tidal portion. Associate species: Platanus occidentalis, Liquidambar styraciflua, Ulmus Americana, Acer rubrum. Understory chiefly Lindera benzoin. Higman I043. 
Java Farm. One station in old field near junction of old entrance road and road to springhouse. Hectare 3407. Associate species: Liquidambar styraciflua. Higman 389.

Syringa vulgaris L.

$$
\text { Syringa }
$$

Common Lilac

(Lilac)

Ivy Neck. One station: yard of reconditioned house near parking area. Hectare 4720. Cultivated. Higman II45.

Ligustrum vulgare L.

\section{Ligustrum}

\section{European Privet}

(Privet)

Java Farm. One station: main building area. Hectare 2580. Persistent after cultivation. Higman 828 .

\section{GENTIANACEAE}

I. Leaves alternate, mostly petioled, compound or crenate. Expected (Menyanthes, Nymphoides).

I. Leaves opposite, sessile, simple, entire.

2. Style filiform; corolla rotate. (Also, corolla pink, 5-parted; leaves thin, cordateovate, clasping) .....................................

2. Style short to absent; corolla campanulate.

3. Calyx lobes 2, resembling leaves. (Also, corolla white, 4-lobed; leaves thick, cuneate-obovate) ......................... Obolaria

3. Calyx 4- or 5-parted, not resembling leaves. Expected (Gentiana, Bartonia).

\section{Sabatia angularis (L.) Pursh}

\section{Sabatia}

(Sabatia angularis (L.) Pers.)

Rosepink

(Rose-pink, Bitter-bloom)

Ivy Neck. One station: along abandoned road through central Scaffold Peninsula. Hectare 5722. Canopy of Quercus alba, Quercus velutina, Liriodendron tulipifera. Associate species: Aureolaria virginica, Vernonia glauca, Amphicarpa bracteata. Higman 964 . 


\section{Obolaria}

Obolaria virginica L.

(Pennywort)

Java Farm. One station: mature forest west of Muddy Creek and south of old entrance road. Canopy of Fagus grandifolia, Quercus alba, Carya tomentosa, Quercus velutina, Liriodendron tulipifera. Higman 653.

\section{APOCYNACEAE}

Apocynum

Apocynum cannabinum L.

Indianhemp

(Dogbane, Indian Hemp)

Java Farm. Infrequent in old fields. Associate species: Solidago altissima, Rubus sp., Lonicera japonica, Rhus radicans. Higman 232.

\section{ASCLEPIADACEAE}

Asclepias

I. Corolla red to purple; hoods ( 5 in a crown on tube of stamens) orange or purple.

2. Tidal stream habitat; umbels several, forming a corymb. (Also, leaves tapering to tip; stems and lower leaf surfaces glabrous or nearly so) ...... A. incarnata

2. Moist to dry forest habitat; umbels I or few, scattered. Expected (A. tuberosa, A. rubra).

I. Corolla and hoods greenish, yellowish, white, or tinged purple.

3. Leaves narrowly linear, with revolute margins. Expected (A. verticillata).

3. Leaves lanceolate to oblong or obovate.

4. Leaves membranaceous, long-attenuate. Expected (A. quadrifolia).

4. Leaves firm, thicker, blunt-tipped.

5. Leaves sessile and clasping at base. Expected (A. amplexicaulis).

5. Leaves short-petioled, not clasping.

6. Leaves glabrous, tapering to petioles; flowers 8-I2 $\mathrm{mm}$ long, white with purple center. Expected (A. variegata).

6. Leaves minutely tomentose below, rounded at base; flowers $12-25 \mathrm{~mm}$ long, hoods greenish to whitish or purplish ............ A. syriaca

Asclepias incarnata L. ssp. pulchra (Ehrh. ex Willd.) Woodson Swamp Milkweed (Asclepias incarnata L., var. pulchra (Ehrh.) Pers.)

Java Farm. Infrequent along tidal portion of Muddy Creek. Associate species: Typha latifolia, Smilax rotundifolia, Aster subulatus, Scirpus validus. Higman 268. 
Java Farm. Moderately abundant along old entrance road near main building area and in grassy fields south of main buildings. Associate species: Cirsium discolor, Rubus sp., Erigeron canadensis, Verbascum blattaria. Higman 359, 825.

\section{CUSCUTACEAE, CONVOLVULACEAE}

I. Parasitic; stem yellowish or reddish; leaves reduced to scales; flowers tiny,

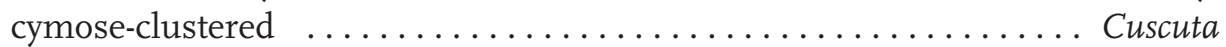

I. Not parasitic; stem green; leaves normal; flowers much larger, few-clustered to solitary.

2. Stigmas 2, linear-filiform to subulate or slenderly ovoid ...... Convolvulus

2. Stigma I, capitate or with $2-3$ globular lobes $\ldots \ldots \ldots \ldots \ldots$ Ipomoea

\section{Cuscuta}

Cuscuta gronovii Willd. ex Schult.

(Cuscuta gronovii Willd.?)

Scaldweed

(Dodder, Strangleweed)

Java Farm and Ivy Neck. Infrequent to moderately abundant in salt marshes. Parasitic on Iva frutescens and Baccharis halimifolia. Higman 62.

Calystegia sepium (L.) R. Br. ssp. sepium

Calystegia

(Convolvulus)

(Convolvulus sepium L.)

Hedge False Bindweed

(Hedge Bindweed, Wild Morning-glory)

Ivy Neck. One station: salt marsh at southwest end of Scaffold Peninsula, on mud flat adjacent water. Hectare 576I. Associate species: Panicum virgatum, Acnida cannabina, Gerardia maritima. Higman 8I4, 969.

\section{Ipomoea}

I. Stem and sepals glabrous, tips of sepals obtuse or blunt; stigma lobes or 2; locules of capsule 2. (Also: corolla white with purple in tube, 4.5 to $8 \mathrm{~cm}$ long; leaves unlobed or with 2 short, rounded basal lobes) ........... I. pandurata

I. Stem and sepals pubescent, tips of sepals acute to linear or bristle-tipped; lobes of stigma and locules of capsule 3 ( 2 in I. lacunosa).

2. Sepals densely yellow-villous near base, short-hirsute above, tapering to long linear tips; leaves densely hirsute on both sides, the lobes, if present, with narrow U-shaped sinuses . . . . . . . . . . . . . . . hederacea

2. Sepals moderately to sparsely pilose, with broadly acute or bristle tips; leaves strigose or sparsely pilose, the lobes, if present, with broadly obtuse sinuses. 
3. Sepals moderately hirsute at base, sparsely so above, with broadly acute tips; peduncles long; leaves never lobed, strigose on both sides......... .................................... . purpurea

3. Sepals sparsely pilose, with bristle tips; peduncles short; leaves sparsely pilose, the lobes, if present, with broadly obtuse sinuses. Expected (I. lacunosa).

Ipomoea pandurata (L.) G. Mey.

(Ipomoea pandurata (L.) G. F. W. Mey.)
Man of the Earth

(Wild Potato-vine)

Ivy Neck. One station: plowed, fallow cornfield on Cheston Peninsula. Higman IO28.

\section{I. hederacea Jacq.}

(I. hederacea (L.) Jacq.)

Ivyleaf Morning-glory

(Blue Morning-glory)

Ivy Neck. Moderately abundant in cornfields. Associate species: Barbarea vulgaris, B. verna, Cerastium vulgatum, etc. Higman 999, I007, I0I6.

I. purpurea (L.) Roth

(I. purpurea (L.) Roth.)
Tall Morning-glory

(Common Morning-glory)

Java Farm and Ivy Neck. Infrequent along unshaded roadsides, and in cornfields at Ivy Neck. Associated with I. hederacea above. Higman I02I, Io64.

\section{BORAGINACEAE}

I. Ovary unlobed, surmounted by the style. (Also, leaves oval, $2-5 \mathrm{~cm}$ long, petioled; spikes bractless, 5-12 cm long; sepals 2-3 mm long). Expected (Heliotropium).

I. Ovary deeply lobed, the I-locular divisions forming nutlets around the base of the style.

2. Attachment of nutlet strongly plug-like, leaving a pit on the gynobase (enlarged receptacle). (Also, corolla campanulate or rotate, blue, or yellowish in Symphytum). Expected (Lycopsis, Borago, Symphytum).

2. Attachment of nutlet flat or concave, leaving no pit.

3. Stigmas 2 or style bifid. (Also, leaves linear-elliptic to narrowly oblanceolate). Expected (Echium, Onosmodium, Lithospermum).

3. Stigma I, simple.

4. Nutlets attached near the apex, strongly divergent. (Also, leaves lanceolate to broadly ovate, IO-I5 $\mathrm{cm}$ long, very bristly, the cauline sessile (clasping in species collected); racemes paniculate; carpels very hirsute; corolla white in species collected ............... Cynoglossum 4. Nutlets attached near the middle or base, erect and parallel. 
5. Flowers of the raceme not subtended by leafy bracts; nutlets smooth. (Also, plant not over $5 \mathrm{dm}$ high; cauline leaves sessile, narrowly oblanceolate-spatulate, very pilose; calyx somewhat irregular; corolla blue or white, $\mathrm{I}-3 \mathrm{~mm}$ broad) $\ldots \ldots \ldots \ldots \ldots \ldots$. . . . . . . .

5. Flowers, at least the lower, with leafy bracts; nutlets prickly, rough, or wrinkled. (Also, cauline leaves elliptic to ovate, up to $2 \mathrm{dm}$ long, smoothish, petioled).

6. Nutlets covered with hooked prickles. (Also, racemes about I dm long, with small bracts; nutlets globose, $3 \mathrm{~mm}$ long ..... Hackelia

6. Nutlets wrinkled, not prickly. (Also, flowers in scorpioid cymes; corolla blue, I-2 cm long). Expected (Mertensia).

Cynoglossum virginianum L.

\section{Cynoglossum}

Wild Comfrey

(Beggar's-lice)

Java Farm. Infrequent to moderately abundant in mature forest south of old entrance road and west of Muddy Creek. Canopy of Fagus grandifolia, Quercus spp., Liriodendron tulipifera, Carya tomentosa, etc. Associate species: Podophyllum peltatum, Claytonia virginica, Galium aparine, Orchis spectabilis, Viola papilionacea, etc. Higman 666.

Myosotis

Myosotis verna Nutt.

Spring Forget-me-not

(species not in Britton and Brown)

(Scorpion-grass Forget-me-not)

Ivy Neck. One station: abandoned grassy field on Scaffold Peninsula. Associate species: Trifolium hybridum, Trifolium pratense, Oenothera fruticosa, Hypericum perforatum, Eupatorium serotinum, etc. Higman 1085.

\section{Hackelia}

Hackelia virginiana (L.) I.M. Johnst.

(Hackelia virginiana (L.) Johnst.)

Beggarslice

(Stickseed Beggar' s-lice)

Java Farm. Infrequent; principal station in small stream valley on Rhode River shore, slightly below pier. Hectare 3519. Canopy of Robinia pseudoacacia, Liquidambar styraciflua, Ulmus americana. Associate species: Lonicera japonica (dominant species of ground layer), Verbesina occidentalis. Rich collecting area. Higman 674 .

\section{VERBENACEAE}

Verbena

I. Spikes panicles at apices of stems and branches; corolla limb 2 to $4.5 \mathrm{~mm}$ broad.

2. Spikes very slender (also interrupted in all species collected); fruiting calyces remote. 
3. Leaves obtuse to subacute, pinnatifid or deeply incised; calyx glandular. Expected (V. officinalis).

3. Leaves acuminate, regularly serrate; calyx not glandular. (Also, corolla white to bluish, with limb $2 \mathrm{~mm}$ broad).

4. Leaves narrowly ovate, 3 to $13 \mathrm{~cm}$ long, serrate-dentate, harshly scabrous above; fruiting calyx 2.5 to $3 \mathrm{~mm}$ long, its lanceolate lobes converging into an acute beak above the schizocarp (pericarp which splits into nutlets); nutlets I to I.3 $\mathrm{mm}$ long, reticulate above ........... V. scabra

4. Leaves oblong-ovate to lanceolate, 8 to $20 \mathrm{~cm}$ long, doubly crenateserrate, strigose on veins beneath; fruiting calyx 2 to $2.3 \mathrm{~mm}$ long, its deltoid subulate-tipped lobes equal to or shorter than the schizocarp; nutlets I.5 to $2 \mathrm{~mm}$ long, not reticulate ............ V. urticifolia

2. Spikes thicker, pencil-like, continuous; fruiting calyces imbricated. (Also, corollas violet-blue; mature calyx overtopping the schizocarp, with incurved

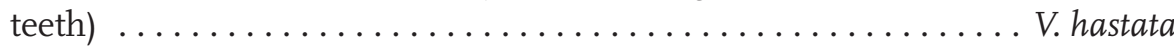

I. Spikes single or in 3's at tips of stems or branches; corolla limb 5 to $6 \mathrm{~mm}$ broad. (Also, in species collected, leaves narrowly lanceolate to oblanceolate, 3 to Io $\mathrm{cm}$ long, serrate; fruiting calyx 4 to $5 \mathrm{~mm}$ long, with acuminate lobes; corolla purple) .................................. V. simplex

Verbena scabra Vahl

Sandpaper Vervain

(Harsh Vervain)

Java Farm. One station: along old entrance road. Canopy of Quercus alba, Fagus grandifolia, and Liriodendron tulipifera. Higman 897.

V. urticifolia L.

White Vervain

Java Farm. One station: western end of main building area. Hectare 2489. Near wooden shed among dense Rhus radicans and Campsis radicans. Higman 4.

V. hastata L.

Swamp Verbena

(Blue Vervain, Simpler's Joy)

Java Farm. One station: clump of trees in Hog Island salt marsh. Hectare 3582. Canopy of Quercus falcata, Quercus stellata, Prunus serotina, Liquidambar styraciflua. Associate species: Panicum virgatum, Osmunda cinnamomea, Juncus dichotomus. Higman 305.

V. simplex Lehm.

(V. simplex Lehm. (V. angustifolia Michx.))

Narrowleaf Vervain

(Vervain)

Ivy Neck. One station: southern part of abandoned grassy field. Hectare 4772. Associate species: Rudbeckia serotina, Trifolium hybridum, Ambrosia artemisiifolia, Oenothera fruticosa. Higman 8I5. 


\section{LAMIACEAE \\ (LABIATAE)}

I. Ovary 4-lobed, not deeply divided.

2. Corolla regular, with 5 subequal lobes. (Also, flowers solitary at ends of terminal pedicels; stamens long-exerted; stem glandular-pubescent) ... Trichostema

2. Corolla irregular, the upper lip apparently wanting . . . . . . . Teucrium

I. Ovary deeply 4-divided. (Also, corolla irregular, with upper lip present or, if regular, 4-lobed).

3. Stamens included in the corolla tube, not directly visible. Expected (Marrubium).

3. Stamens exerted from the corolla tube, directly visible.

4. Upper lip of corolla helmet-shaped or concave. (Also, stamens always 4, ascending under the corolla lip, exceeding it only in Agastache).

5. Calyx with a helmet-like projection on the upper side, closed in fruit. (Also, flowers blue to purple, usually in leafy-bracted racemes or panicles but axillary in one species) $\ldots \ldots \ldots \ldots \ldots$. . . . . . . .

5. Calyx without a helmet-like projection on the upper side, open in fruit except in Prunella.

6. Stem prostrate to ascending; leaves cordate to reniform at base. (Also, flowers lilac to blue-violet).

7. Flowers distinctly pediceled, usually 3 per axil; upper stamens longer than the lower; corollas blue-violet ......... Glechoma

7. Flowers sessile, in dense terminal and axillary verticils; upper stamens shorter than the lower; corollas pink-or red-violet Lamium

6. Stem erect; leaves acute, rounded, or shallowly cordate at base.

8. Flowers axillary. Expected (Leonurus, Ballota).

8. Flowers in terminal spikes, clusters, or heads; if axillary, the subtending leaves reduced and clustered into a terminal spike.

9. Spike very dense (may be somewhat interrupted in Nepeta).

Io. Calyx distinctly 2-lipped, closed in fruit. (Also, flowers blueviolet, in clusters of 3 subtended by round bracts) Prunella

Io. Calyx nearly regular, open in fruit. Expected (Nepeta, Agastache). See Appendix I.

9. Spike loose or interrupted. (Also, calyx regular; flowers pale purple or pink).

II. Plant glabrous; flowers single in axils of small bracts. Expected (Physostegia).

II. Plant pubescent; flowers in small, dense cymes subtended

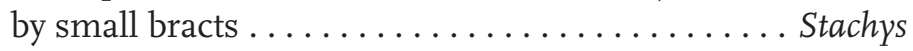

4. Upper lip of corolla not helmet-shaped or concave.

I2. Flowers in dense cymes or heads. (Also, corolla purple to white) ....

Pycanthemum 
I2. Flowers in panicles, racemes, spikes, or clusters, inflorescence terminal or axillary.

I3. Flowers in loose terminal racemes or panicles. (Also, leaves ovate or ovate-oblong, serrate, acuminate, with long petioles).

I4. Plant annual, without a rootstock; calyx villous; corolla purple (at Chesapeake Bay Center) or white; stamens 4. (Also, leaves purple beneath) .................. Perilla

I4. Plant perennial, with large woody rootstock; calyx nearly glabrous, minutely glandular; corolla pale yellow; stamens 2 . (Also, one of the 5 corolla lobes much enlarged and deeply fringed; stamens and style long-exserted) ...... Collinsonia

I3. Flowers in spikes or clusters, terminal or axillary.

I5. Stamens 2.

I6. Flowers axillary, in dense clusters.

I7. Corolla regular, 4-lobed. (Also, leaves coarsely dentate, acuminate at both ends; strong mint odor) ... . Lycopus

I7. Corolla irregular, 2-lipped. Expected (Hedeoma, Monarda, and Blephilia).

I6. Flowers in an interrupted spike. (Also, in species collected, leaves basal, lyrate-pinnatifid) ......... Salvia

I5. Stamens 4 .

I8. Corolla regular, 4-lobed. (Also, in species collected, flowers in an interrupted spike; strong mint odor) ...... Mentha

I8. Corolla irregular, 2-lipped. (Also, flowers axillary). Expected (Melissa, Satureja)

Trichostema

Tricbostema dichotomum L.

Forked Bluecurls

(Bluecurls, Bastard Pennyroyal)

Java Farm. Infrequent in old fields, more abundant near young Pinus virginiana stands invading these fields. Hectares 3513, 3535. Associate species: Solidago juncea, Solidago nemoralis. Higman 378, 462 .

\section{Teucrium}

Teucrium canadense L.

Canada Germander

(Germander, Wood-sage)

Java Farm. One station: new entrance road. Canopy of mature Quercus alba, Fagus grandifolia, Liriodendron tulipifera. Higman 9i8. 


\section{Scutellaria}

I. Racemes not I-sided; base of stem without stolons.

2. Cauline leaves round-to oblong-ovate or the lower deltoid, all dentate or serrate. 3. Principal leaves cordate at base, villous. Expected S. ovata).

3. Principal leaves not cordate, if shallowly so, not villous.

4. Principal leaves rhombic-ovate, 3 to $6 \mathrm{~cm}$ long. (Also, lower leaves slender-petioled, median and upper ones cuneate to short-winged petioles; 2 to 5 pairs of leaves below the single or panicled terminal racemes; corolla $\mathrm{I} .4$ to $2 \mathrm{~cm}$ long) .............. S. elliptica

4. Principal leaves ovate or ovate-oblong, 4 to $\mathrm{I} 4 \mathrm{~cm}$ long. Expected (S. serrata, S. incana).

2. Cauline leaves ovate and crenate below, oblong to linear-lanceolate and entire above, successively narrower to summit of stem. (Also, petioles of upper leaves short, not winged, 3 to 8 pairs of leaves below the terminal raceme or leafy panicle of them) .............. integrifolia

I. Racemes I-sided; base of stem with creeping rhizomes developed from stolons. (Also, in species collected, racemes in upper axils as well as terminal, their bracts not minute).

5. Stolons not forming tubers; lower leaves regularly serrate, with slender petioles; stem with incurved-ascending pubescence at least on the angles; leaves round to subcordate to base, acuminate to tip ........ S. lateriflora

5. Stolons, leaves, or stem pubescence otherwise. Expected (S. parvula, S. nervosa, S. saxatilis).

Scutellaria elliptica Muhl. ex Spreng. (Scutellaria elliptica Muhl.)

Hairy Skullcap

Ivy Neck. Infrequent in west-central Scaffold Peninsula. Canopy of Quercus alba, Quercus velutina, Liriodendron tulipifera, Liquidambar styraciflua, Carya tomentosa. Higman I093.

S. integrifolia L.

Helmet Flower

(Skullcap)

Ivy Neck. Infrequent in west-central Scaffold Peninsula. Associated with S. elliptica above.

Java Farm. One station: south-facing slope east of Muddy Creek, adjacent north boundary of Center. Hectares 2458, 2459. Partial canopy of Liquidambar styraciflua, Pyrus malus, and Liriodendron tulipifera. Dense Rhus radicans on ground, with few grassy patches. Higman 545, 849 . 
Java Farm. Infrequent in freshwater marsh at western apex of triangular area west of old Muddy Creek Road. Hectare 2470. Higman 405.

\section{Glechoma}

Glechoma hederacea L.

Ground Ivy

(Glechoma hederacea L. var. micrantha Moricand) (Ground-ivy, Gill-over-the-ground)

Java Farm. One station: gravel fill of old Muddy Creek Road, beside stream culvert south of entrance gate. Hectare 249I. Shaded by adjacent canopy of Fagus grandifolia; growing among dense Lonicera japonica. Higman 566.

\section{Lamium}

I. Upper or bracteal leaves sessile, reniform; flowers 6-Io per whorl; calyx teeth

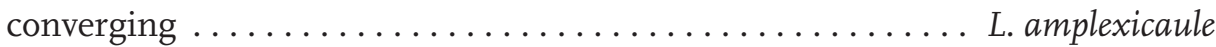

I. Upper or bracteal leaves petioled, ovate-subrhombic; flowers 3-7 per whorl; calyx teeth divergent $\ldots \ldots \ldots \ldots \ldots \ldots \ldots \ldots \ldots \ldots \ldots \ldots \ldots \ldots \ldots \ldots$. purpureum

Lamium amplexicaule L.

Henbit Deadnettle

(Henbit, Dead-nettle)

Java Farm. Infrequent around parking apron, main building area. Hectare 2580. Associate species: Juncus tenuis.

Ivy Neck. Infrequent to moderately abundant in cornfields and around old tobacco barn near entrance road. Hectare 4658. Associate species: Draba verna, Stellaria media, Cerastium vulgatum. Higman 553.

L. purpureum L.

Purple Deadnettle

(Purple Dead-nettle)

Java Farm. One station: edge of old Muddy Creek Road, on top of stream culvert south of entrance gate. Hectare 249I. Near station for Glechoma hederacea, but drier. Higman 565 .

Prunella

Prunella vulgaris L.

Common Selfheal

(Selfheal, Carpenter-weed)

Java Farm. Infrequently along Fox Point and old entrance roads. Partial shading on springhouse road by Celtis occidentalis, Liquidambar styraciflua, and Prunus serotina. Associate species: Melilotus alba. Higman 20, II8. 
Java Farm. One station: salt marsh at northeast corner of Farm, above pier. Hectare 3620. Flooded at high tide. Associate species: Spartina cynosuroides, Baccharis halimifolia, Limonium carolinianum, Aster subulatus. Higman 2I6.

\section{Pycnanthemum}

(Pycanthemum)

Pycnanthemum flexuosum (Walter) Britton,

Sterns \& Poggenb.

(Pycanthemum flexuosum (Walt.) BSP.)

Appalachian Mountainmint

(Mountain-mint, Basil)

Java Farm. One station: old field dominated by Aster spp., Rubus spp., Solidago altissima, Rhus radicans, Lonicera japonica, etc. Hectare 350I. Higman 364.

\section{Perilla}

Perilla frutescens (L.) Britton

Beefsteakplant

(Perilla frutescens (L.) Britt.)

(Beefsteak-plant)

Java Farm. One station: grassy triangle at entrance gate to old Muddy Creek Road. Hectare 2463. Associate species: Cyperus strigosus, Duchesnea indica, Fragaria virginiana, Ranunculus bulbosus, Daucus carota.

Ivy Neck. One station: tractor trail to artificial pond, above source of Cheston Creek Drainage. Hectare 472I. Associate species: Mentha spicata, Ambrosia trifida, Galinsoga ciliata. Higman I03I, IO6I.

Collinsonia canadensis L.

\section{Collinsonia}

Richweed

(Horse-balm, Stoneroot)

Java Farm. One station: mature forest south of old entrance road. Hectare 2484 . Canopy of Quercus alba, Fagus grandifolia, Liriodendron tulipifera, etc. Higman I206.

Lycopus virginicus L.

$$
\text { Lycopus }
$$

Virginia Water Horehound (Water-horehound, Bugleweed)

Java Farm. Infrequent along bank of Muddy Creek, upstream from old entrance road. Hectare 2467. Canopy of Fagus grandifolia, Platanus occidentalis, Quercus alba. Understory of Lindera benzoin.

Also infrequent in freshwater part of Fox Creek marsh. Hectare 3516. Higman 977, I209. 
Salvia

Salvia lyrata L.

Lyreleaf Sage

(Cancerweed, Lyre-leaved Sage)

Java Farm. One station: mature forest along old entrance road, near wellhouse. Hectare 2487. Canopy of Fagus grandifolia, Quercus alba, Liriodendron tulipifera, etc. Higman 8I9.

Mentha spicata L.

Mentha

Ivy Neck. One station: partly shaded border of tractor trail to artificial pond north of Cheston Peninsula, near source of Cheston Creek drainage. Hectare 47I2. Associate species: Ambrosia trifida, Chondrilla juncea, Solanum carolinense, Cichorum intybus. Higman I026.

\section{SOLANACEAE}

I. Plant a shrub. Expected (Lycium).

I. Plant a forb or vine.

2. Corolla funnelform, 7 to Io $\mathrm{cm}$ long, white with purple tinge; fruit a large,

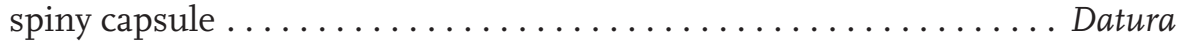

2. Corolla rotate to campanulate, much shorter, white or yellow (sometimes purple, but not in species collected); fruit a berry.

3. Anthers forming a tube around the style; calyx deeply 5-parted, not inflated over berry in fruit . . . . . . . . . . . . . . . . Solanum

3. Anthers separate; calyx 5-lobed only at the summit, inflated and loosely covering the berry in fruit.

4. Sepals united, calyx with round or cordate base in fruit; corolla yellow or yellowish-green, with dark center; ovary 2-locular; berry juicy ... . Physalis

4. Sepals free, sagittate-based; corolla pale blue; ovary 3-to 5-locular; berry dry. Expected (Nicandra).

\section{Datura}

\section{Datura stramonium L.}

Jimsonweed

Java Farm. One station: trash pile near ruined house, main building area. Hectare 2581. Associate species: Melilotus alba, Aster spp., Eleusine indica, Solanum nigrum, and Amaranthus spinosus.

Ivy Neck. Infrequent: edge of cornfield near old barn south of artificial pond, north of Cheston Peninsula. Hectare 4733. Associate species: Eleusine indica, Abutilon theophrasti, Potentilla norvegica, Ipomoea spp. Higman 50I, I008. 


\section{Solanum}

I. Calyx lobes very spiny, covering the berry. Expected (S. rostratum, S. sisymbriifolium).

I. Calyx not spiny, not covering the berry.

2. Plant a woody vine or climbing shrub; berries red. (Also, corolla violet; leaves often basally lobed). Expected (S. dulcamara).

2. Plant herbaceous, not a vine; berries yellow, green, or black.

3. Stem and leaf veins with yellow spines; berry orange-yellow. (Also, corolla violet or white; stein hirsute) . . . . . . . . . . . carolinense

3. Stem and leaf veins spineless; berries black or green. (Also, corolla white). 4. Stem glabrous; berries black.

5. Leaves thin, translucent; berries shiny. Expected (S. americanum). Appendix I.

5. Leaves rather thick, opaque; berries dull ............. S. nigrum 4. Stem very spreading-hirsute; berries green ........ S. sarachoides

Solanum carolinense L.

Carolina Horsenettle

(Horse-nettle)

Java Farm. Infrequent in old fields, especially in the vicinity of main buildings. Associate species: Poa pratensis, Agrimonia parviflora, Verbascum blattaria, Cirsium discolor, Asclepias syriaca, Gnaphalium obtusifolium. Higman 45, I33.

S. nigrum L.

Black Nightshade

Java Farm. One station: rubbish pile near ruined house, main building area. Hectare 2580. Associate species: Datura stramoneum, Amaranthus spinosus, Eleusine indica. Higman 498.

\section{S. physalifolium Rusby}

Hoe Nightshade

(S. sarachoides Sendtner)

Java Farm. Infrequent on recently bulldozed area at main buildings. Hectare 2580. Higman I2I6.

I. Plants annual, without rhizomes.

\section{Physalis}

2. Plant glabrous, glabrate, or puberulent. Expected ( $P$. ixocarpa).

2. Plant villous. (Also, leaves ovate, obliquely rounded or cordate at base; berry

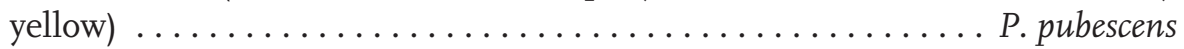

I. Plants perennial, with deep-seated rhizomes. (Also, corolla yellow, often with dark center; berry red, yellow, or green).

3. Leaves, petioles, and pedicels puberulent; calyx glabrous when mature .... .P. subglabrata 
3. Leaves, petioles, pedicels, and calyx villous or hirsute.

4. Leaves cordate or broadly rounded at base; pubescence very dense, often glandular. Expected (P. heterophylla).

4. Leaves cuneate or gradually tapering to base; pubescence not very dense, not glandular.

5. Leaves oblanceolate, oblong, or subspatulate; fruiting calyx not depressed at base; berry yellow or green. Expected ( $P$. lanceolata).

5. Leaves ovate or ovate-lanceolate; fruiting calyx deeply depressed at base;

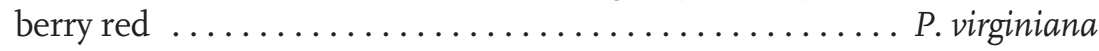

Physalis pubescens L.

Husk Tomato

(Ground-cherry)

Java Farm. Collected by David Hammond.

\section{P. longifolia Nutt. var. subglabrata (Mack. \& Bush) Cronquist Longleaf Groundcherry (Physalis subglabrata Mackenz. and Bush) (P. longifolia Nutt.) \\ (Ground-cherry)}

Java Farm. One station: new entrance road, near culvert of Fox Creek drainage. Hectare 2573. Partial canopy of Juglans nigra and Liquidambar styraciflua. Associate species: Geum canadense, Duchesnea indica, Agrimonia microcarpa, Panicum clandestinum. Higman I034, II8I.

P. virginiana Mill.

Virginia Groundcherry

(Ground-cherry)

Ivy Neck. One station: abandoned grassy field on Scaffold Peninsula. Hectare 4772. Associate species: Campsis radicans, Oenothera fruticosa. Higman 1088.

\section{SCROPHULARIACEAE}

I. Plant a tree; leaves I.5 to $4 \mathrm{dm}$ wide; cordate. (Also, leaves opposite, tomentose; petioles and young stem hollow except at the nodes) .......... Paulownia

I. Plant herbaceous or only slightly woody; leaves much narrower, not cordate.

2. Corolla nearly regular.

3. Leaves opposite (whorled in Veronicastrum), the upper sometimes alternate; stamens 2 or 4 , the filaments not bearded.

4. Corolla yellow or roseate to red-purple, campanulate to funnelform.

(Also, plant dries black) ....................... Gerardia

4. Corolla bluish to dark blue-purple, rotate or salverform.

5. Corolla rotate, almost tubeless; capsule obcordate or notched at apex; flowers axillary or in terminal racemes .............. Veronica

5. Corolla salverform; capsule blunt or acute at apex; flowers in spikes. Expected (Buchnera, Veronicastrum). 
3. Leaves all alternate; stamens 5 , with 3 or more filaments bearded. (Also, flowers in spikes or racemes, yellow or white) ........... Verbascum 2. Corolla 2-lipped.

6 . Leaves, or at least the upper ones, alternate.

7. Leaves pinnately cleft or dissected. Expected (Castilleja, Pedicularis).

7. Leaves entire or palmately lobed.

8. Flowers in racemes or spikes; leaves linear to lanceolate, entire .... Linaria

8. Flowers axillary; leaves broadly ovate, undulate or lobed. Expected (Kickxia, Cymbalaria).

6. Leaves all opposite.

9. Flowers in racemes, spikes, panicles, or cymes. Expected (Chelone, Penstemon).

9. Flowers solitary in axils of leaves or bracts.

Io. Pedicels with 2 bracts just beneath the calyx. (Also, stamens 2; flowers small, yellow or white) $\ldots \ldots \ldots \ldots \ldots$. Gratiola

Io. Pedicels naked at summit. Expected (Mimulus, Micranthemum, Lindernia, Bacopa).

\section{Paulownia}

Paulownia tomentosa (Thunb.) Siebold \& Zucc. ex Steud.

Princesstree

(Paulownia tomentosa (Thunb.) Steud.)

(Princess-tree)

Java Farm. One station: young tree on rubbish pile near northwest corner of main building area. Hectare 2489. Associate species: Populus grandidentata.

Ivy Neck. One station: young tree on west shore of large cut-off pond on Cheston Peninsula. Hectare 5800. Associate species: Phytolacca americana. Higman I054.

\section{Agalinis, Aureolaria}

(Gerardia)

I. Corolla pink or purple; capsules rounded at summit, often mucronate.

2. Terrestrial habitat. Expected (G. purpurea, G. obtusifolia).

2. Salt marsh habitat. (Also, stem 0.5 to $3 \mathrm{dm}$ high, glabrous; leaves linear) ... .................................... maritima

I. Corolla yellow, may have reddish tinge, dries black; capsules acute.... G. virginica

Agalinis maritima (Raf.) Raf. var. maritima

Saltmarsh False Foxglove (Gerardia maritima Raf.)

(Gerardia)

Ivy Neck. Infrequent in sandy, halophytic area at Sand Point on Cheston Peninsula (hectare 486I) and in salt marsh on southwest shore of Scaffold Peninsula (hectare 576I). Associate species: Acnida cannabina, Solidago sempervirens, Panicum virgatum. Higman 972. 
Aureolaria virginica (L.) Pennell

(G. virginica (L.) BSP.)

(Aureolaria virginica (L.) Pennel)

(Aureolaria flava (L.) Farw.)
Downy Yellow False Foxglove

(Downy False Foxglove)

Java Farm. One station: ruined house at Fox Point. Hectare 3558. Canopy of Quercus prinus and Quercus alba. Associate species: Gaylussacia baccata, Vaccinium vacillan.

Ivy Neck. Infrequent along southern shore of Scaffold Peninsula. Hectare 5762 to 5764. Canopy and associate species as at Java Farm. Also one station along abandoned road through central forest on Scaffold Peninsula. Hectare 5732. Canopy of Quercus alba, Fagus grandifolia, Liriodendron tulipifera, Carya tomentosa, etc. Higman 350.

\section{Veronica}

I. Flowers sessile; sepals lanceolate, short-pilose, differing in length by about I mm; corolla blue-violet; lower leaves round or oval, dentate, the upper ones lanceolate to linear $\ldots \ldots \ldots \ldots \ldots \ldots \ldots \ldots \ldots \ldots \ldots \ldots \ldots$ arvensis

I. Flowers pediceled; sepals cordate-ovate, ciliate, equal; corolla lilac to blue; all leaves reniform to suborbicular, with 2 or 4 big lateral teeth ...... V. hederaefolia

Veronica arvensis L.

Corn Speedwell

Java Farm. Infrequent, along road to springhouse. Hectare 34I7. Associate species: Valerianella olitoria, Duchesnea indica, Viola spp., Melilotus alba. Higman 608.

V. hederifolia L.

(V. hederaefolia L.)
Ivyleaf Speedwell

(Ivy-leaved Speedwell)

Java Farm. Infrequent along road to springhouse. Very calcareous soil. Hectare 34I7. Associate species: Valerianella olitoria, Veronica arvense, Duchesnea indica. Higman 564, 629.

Also along bank of Muddy Creek, upstream from culvert of old entrance road. Hectare 2467. Aspect dominant species of ground layer in early spring. Canopy of Fagus grandifolia, Platanus occidentalis, Quercus alba. Understory of Cornus florida, Lindera benzoin.

\section{Verbascum}

I. Stems and leaves tomentose; leaves entire, decurrent down the stem; flowers in a dense, cylindrical spike $\ldots \ldots \ldots \ldots \ldots \ldots \ldots \ldots \ldots \ldots \ldots$. thapsus

I. Stems and leaves glabrous, or the upper stem glandular-pubescent; leaves doubly serrate, the lower petioled, the upper partly clasping; flowers in a loose

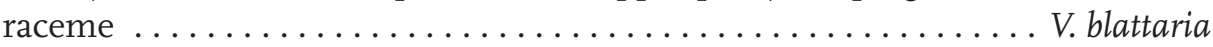


Java Farm. Infrequent in a variety of old field and roadside habitats, especially near main building area. Principal hectares 3409, 3500, 2590. Associate species: Asclepias syriaca, Cirsium discolor, Solanum carolinense, Poa pratense (dense turf), Verbascum blattaria, Melilotus alba, Daucus carota, Gnaphalium obtusifolium.

Ivy Neck. Infrequent in side of steep bank along shore of Rhode River above Sand Point. Associate species: most of above plus Juncus tenuis, Atriplex patula, Plantago lanceolata, Arctium minus, Amorpha fruticosa, Rhus typhina, Solidago sempervirens. Higman 43.

\section{V. blattaria L.}

(V. blattaria L., V. blattaria forma albiflora (Don) House)

Moth Mullein

(Moth-Mullein)

Java Farm. Infrequent in old fields south of main building area. Associated with $V$. thapsus above.

Ivy Neck. Infrequent in grassy abandoned field on Scaffold Peninsula. Hectare 4762. Associate species: Trifolium pratense, Trifolium hybridum, Oenothera fruticosa.

Only the white-flowered form occurs at Java Farm; both white- and yellow-flowered forms occur at Ivy Neck, sometimes on the same plant. Higman 47, 789, 8II.

\section{Nuttallanthus \\ (Linaria)}

Nuttallanthus canadensis (L.) D.A. Sutton

Linaria canadensis (L.) Dumont

Canada Toadflax

(Old-field Toadflax)

Ivy Neck. Moderately abundant in cultivated fields and along tractor trails. Associate species: Draba verna, Arabidopsis thaliana, Cerastium vulgatum, Stellaria media, etc. Higman 657.

Gratiola neglecta Torr.

\section{Gratiola}

Clammy Hedgehyssop

(Hedge Hyssop)

Infrequent along both forks of drainage to Cheston Creek estuary, and beside artificial pond. Hectares 4752 and 47I2 respectively. Canopy of Liquidambar styraciflua, Platanus occidentalis, and Diospyros virginiana at the first station; second station partly shaded by nearby canopy. Associate species: Ranunculus sceleratus, Limnobium spongia, Galinsoga ciliata, Glyceria striata. Higman 725, 873. 


\section{BIGNONIACEAE}

\section{Campsis}

Campsis radicans (L.) Seem. ex Bureau

Trumpet Creeper

(Campsis radicans (L.) Seem.)

(Trumpet-vine)

Java Farm. Aggressive invader of old fields; seems to thrive where Rhus radicans and Lonicera japonica are not too abundant. Much less frequent than Rhus radicans in forest.

Ivy Neck. Abundant to moderately abundant in old fields on small peninsula between forks of Scaffold Creek estuary. Abundant in ground layer of forest at source of Scaffold Creek drainage (east fork). Invading abandoned grassy field on Scaffold Peninsula. Higman I.

\section{OROBANCHACEAE}

Epifagus virginiana (L.) W.P.C. Barton

\section{Epifagus}

(Epifagus virginiana (L.) Bart.)

Beechdrops

(Beech-drops)

Java Farm. Moderately abundant under mature Fagus grandifolia canopy, especially west of old Muddy Creek Road. Hectare 2480. Higman 249.

\section{VERBENACEAE}

(PHRYMACEAE)

Phryma

Phryma leptostachya L.

American Lopseed

(Lopseed)

Java Farm. Infrequent along old entrance road near gate to old Muddy Creek Road, hectare 2463. Canopy of Fagus grandifolia, Quercus alba, Liriodendron tulipifera, Quercus coccinea, Liquidambar styraciflua, Carya tomentosa, etc. Associate species: Desmodium paniculatum, Desmodium glutinosum, Elephantopus carolinianus, Agrimonia microcarpa, Viola papilionacea, Smilacina racemosa, etc. Higman 93I.

\section{PLANTAGINACEAE}

I. Spike cylindric.

\section{Plantago}

2. Leaves obovate or spatulate.

3. Leaves scabrous; scape glabrous. (Also, bracts of spike shorter than to equaling calyx) $\ldots \ldots \ldots \ldots \ldots \ldots \ldots \ldots \ldots \ldots \ldots \ldots \ldots \ldots \ldots \ldots \ldots \ldots$ major

3. Leaves villous; scape villous $\ldots \ldots \ldots \ldots \ldots \ldots \ldots \ldots$. virginica 
2. Leaves linear, with margined semiclasping petioles. (Also, bracts of spike linear, much longer than the flowers; bracts, sepals, and scape villous) ....

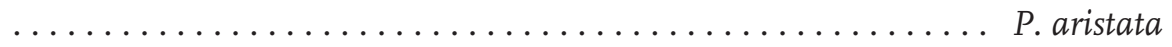

I. Spike ovoid-conic, tapering to apex. (Also, leaves lanceolate to lance-oblong; bracts of spike broadly ovate, scarious, with villous green midribs) ...... P. lanceolate

Plantago major L.

Common Plantain

Java Farm. Infrequent around main buildings and along Fox Point Road. Higman I9.

P. virginica L.

Virginia plantain

(Hoary Plantain)

Ivy Neck. Infrequent in abandoned field on Scaffold Peninsula, and along adjacent tractor trails. Associate species: Hypericum perforatum, Trifolium arvense, Hordeum pusillum, Rumex acetosella, Juncus tenuis, Lepidium virginicum, Trifolium pratense. Higman 692.

P. aristata Michx.

Largebracted Plantain

(Bracted Plantain)

Java Farm. One station: broken pavement along Fox Point Road near sharp left turn onto peninsula. Hectare 3543. Associate species: Eragrostis spectabilis, Festuca myuros.

Ivy Neck. Infrequent along tractor trail beside abandoned field on Scaffold Peninsula. Associated with Plantago virginica above. Higman 806.

P. lanceolata L.

Narrowleaf Plantain

(Ribgrass, English Plantain)

Java Farm. Infrequent around main building area. Hectare 2590. Associate species: Juncus tenuis, Cyperus strigosus, Achillea millefolium, Erigeron canadensis, Triodia flava, Euphorbia maculata. Higman I2, ıо92.

\section{RUBIACEAE}

I. Plant a woody shrub; flowers in large spherical heads ........ Cephalanthus

I. Plant herbaceous; flowers not in heads.

2. Leaves whorled .............................

2. Leaves opposite.

3. Stem prostrate-trailing; leaves evergreen; flowers paired, their ovaries united; fruit a berry. (Also, flowers white; leaves round-ovate; berries red) Mitchella 
3. Stem erect (somewhat procumbent and spreading in Diodia); leaves deciduous; flowers cymose, solitary or clustered, if paired, the ovaries not united; fruit a capsule.

4. Corolla rotate. (Also, flowers sessile in axillary clusters). Expected (Hedyotis). 4. Corolla funnelform or salverform.

5. Flowers axillary, I to 3 per axil, sessile; leaves without stipules ..... ..................................... Diodia

5. Flowers in terminal cymes, or (not in species collected) solitary and peduncled; leaves connected by rounded stipules ........ Houstonia

Cephalanthus occidentalis L.

\section{Cephalanthus}

Common Buttonbush

(Buttonbush)

Java Farm. One station at west end of Phalaris arundinacea meadow, north of main building area. Hectare 2468. Associate species: Juncus effusus, Erechtites hieracifolia, Polygonum sagittatum, Polygonum pensylvanicum.

Ivy Neck. One station on sand bar of cut-off pond on Rhode River shore of Cheston Peninsula. Hectare 4880. Associate species: Sium suave, Rumex verticillatus, Asparagus officinalis, Sambucus canadensis. Higman I75, 947.

\section{Galium}

I. Ovary and fruit smooth, muricate, or granular. Expected (G. verum, G. mollugo, G. obtusum, G. concinnum).

I. Ovary and fruit bristly.

2. Leaves 6 to 8 per whorl; stems weak, reclining.

3. Stem harsh on the angles; leaves 8 per whorl, linear to narrowly oblanceolate ............................. G. aparine

3. Stem smooth; leaves 6 per whorl, narrowly elliptic. Expected (G. triflorum).

2. Leaves 4 per whorl; stems ascending, not reclining.

4. Flowers on distinct pedicels terminating the branches of the panicle. Expected (G. pilosum).

4. Flowers sessile along the loosely divergent branches of the inflorescence. (Also, leaves oval or elliptic) .................... G. circaezans

Galium aparine L.

Stickywilly

(Cleavers, Bedstraw)

Java Farm. Infrequent in bottom of Muddy Creek valley, above tidal portion. Canopy primarily Fraxinus americana. Also one station at shoulder of old Muddy Creek Road, near entrance gate. Associate species: Duchesnea indica, Poa sylvestris, Geranium carolinianum. Higman 95, 639 . 
Java Farm. Infrequent along old entrance road, west of Muddy Creek. Canopy of Fagus grandifolia, Carya tomentosa, Quercus alba, Liriodendron tulipifera, etc. Associate species: Houstonia purpurea, Arrhenatherum elatius. Higman 777.

\section{Mitchella}

Mitchella repens L.

Partridgeberry

(Partridge-berry)

Java Farm. Infrequent to moderately abundant beneath canopy of mature Fagus grandifolia, especially along old entrance road near wellhouse. Hectares 2477, 2478 , and 2487.

Ivy Neck. One station: west shore of Cheston Creek estuary. Hectare 5704. Canopy of mature Quercus prinus. Higman 93, 774.

\section{Diodia}

\section{Diodia teres Walter}

Ivy Neck. One station: tractor trail to grassy abandoned field on Scaffold Peninsula. Hectare 4740. Higman IoI9.

Houstonia purpurea L.

\section{Houstonia}

Java Farm and Ivy Neck. Infrequent in mature hardwood forest, either under canopy of Fagus grandifolia, Quercus alba, Liriodendron tulipifera, etc. or along estuary shorelines under Quercus alba and Quercus prinus. Found along north boundary of Java Farm west of Muddy Creek, on north shore of Fox Point peninsula, along old entrance road west of Muddy Creek, and along west shore of Cheston Creek estuary. Higman 776, 840, 887, 974.

\section{CAPRIFOLIACEAE}

I. Corolla funnelform to campanulate, irregular; style long; stigma capitate.

2. Plant a shrub or woody vine.

3. Corolla funnelform to tubular; ovary 2- or 3-locular; fruit a berry. (Also, plant a woody vine in species collected; flowers fragrant and showy) ....

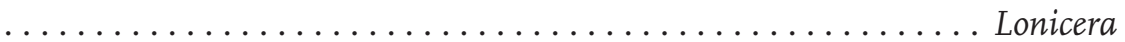

3. Corolla short-campanulate to salverform; ovary 4-locular; fruit a berrylike drupe, with only 2 nutlets maturing .......... Symphoricarpos 
2. Plant herbaceous. (Also, leaves over I dm long; flowers axillary, sessile; corolla tubular, yellowish to dull red; fruit a dry orange-yellow berry) ..... Triosteum

I. Corolla rotate to open-campanulate, regular, deeply 5-lobed; style absent; stigmas I to 5 .

4. Plant a woody shrub or small tree; leaves simple; fruit a drupe with I stone

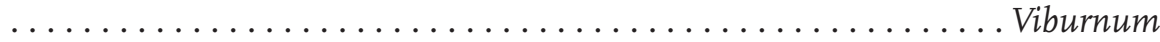

4. Plant a semi-herbaceous shrub, the stems with large white pith; leaves pinnately compound; fruit a berry, with 3 or more seed-like nutlets . . . . Sambucus

Lonicera japonica Thunb.

Lonicera

Java Farm and Ivy Neck. Usually abundant throughout old fields, along roadsides, and as a dense ground cover in both pine and hardwood forest. Associate species: Rhus radicans, Campsis radicans, Vitis vulpina. Conspicuously infrequent (as are its associates) along estuary shores under canopy of Quercus prinus. Higman I2I.

L. japonica Thunb.

(L. japonica, var. chinensis (P. W. Wats) Baker)

Japanese Honeysuckle

Java Farm. Infrequent, in old fields near Fox Point Road and new entrance road. Associated with typical form above. Higman 202,

Symphoricarpos occidentalis Hook.

Symphoricarpos

Western Snowberry

(Snowberry, Wolf berry)

Ivy Neck. Two stations: east and west coasts of Cheston Point forest. Canopy of Pinus taeda, Robinia pseudoacacia, Liriodendron tulipifera. Higman ıogo.

\section{Triosteum}

I. Leaves perfoliate below, the upper tapering to base, glabrous or puberulent above; flowers 3 or 4 per axil ................. perfoliatum

I. Leaves all tapering to base, strigose above; flowers I per axil . . . T. angustifolium

Triosteum perfoliatum L.

Feverwort

(Tinker's-weed, Horse-gentian)

Java Farm. Infrequent to moderately abundant on east- to south-facing slopes overlooking Rhode River, north of Fox Creek drainage. Hectares 35I7, 3518. Canopy of Liquidambar styraciflua, and usually Robinia pseudoacacia and Ulmus americana. Associate species: Verbesina occidentalis.

Also, infrequent in old fields near the above area. Hectares 3507, 3508, 3509. Dominant species: Rhus radicans, Lonicera japonica, Solidago altissima, Rubus spp. Scattered Ulmus americana, Ulmus rubra. Higman 230, 762. 
Ivy Neck. One station: beside abandoned road down Cheston Peninsula. Hectare 5719. Very thin canopy of mature Pinus taeda, moderately dense understory of Liquidambar styraciflua and other hardwoods, very dense ground cover of Rhus radicans and Lonicera japonica. Higman I077.

\section{Viburnum}

I. Leaves palmately 3-lobed, much dotted beneath; petioles stipulate at the base

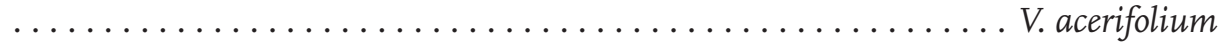

I. Leaves unlobed, orbicular to ovate, not dotted beneath; petioles not stipulate.

2. Leaves coarsely dentate, orbicular to oblong-ovate, usually pubescent . . . . . $\ldots \ldots \ldots \ldots \ldots \ldots \ldots \ldots \ldots \ldots \ldots \ldots$. dentatum

2. Leaves serrulate, ovate, glabrous . V. prunifolium

Viburnum acerifolium L.

Mapleleaf Viburnum (Maple-leaved Viburnum, Arrow-wood)

Java Farm. Infrequent; one station on south-facing slope overlooking tidal portion of Muddy Creek, under canopy of Quercus alba, Quercus velutina, Fagus grandifolia, Liriodendron tulipifera, and Carya tomentosa. Also one station on Hog Island, under canopy of Quercus alba and Quercus prinus. Hectares 3429 and 4502. Higman I00, 457.

V. dentatum L.

Southern Arrowwood

(Southern Arrow-wood)

Java Farm. Locally abundant, in broad valley tributary to tidal portion of Muddy Creek. Hectare 3530. Canopy of Quercus alba, Fagus grandifolia, and Liriodendron tulipifera. Associate species: Viburnum prunifolium, Lindera benzoin.

Also, infrequent in old fields near Fox Point Road. Hectare 3523. Dominant species: Rubus spp., Lonicera japonica, Rhus radicans. Associate species: Diospyros virginiana, Liquidambar styraciflua, Celtis occidentalis. Higman 37I, 455, 5I4.

V. prunifolium L.

Blackhaw

(Black-haw (L), Sweet-haw)

Java Farm and Ivy Neck. Infrequent to moderately abundant along roadsides, in old fields, and under a variety of hardwood canopies, including Juglans nigra and Quercus prinus. More frequent and widespread than either V. acerifolium or V. dentatum. Higman 220, 429, 464, 507, 638. 
Java Farm. Infrequent; stations at main building area, west end of marsh meadow north of main buildings, and in freshwater marsh of Fox Creek drainage. Hectares 2580, 2468, and 3516, respectively. Station at main buildings may be persistent from cultivation. Associate species at both other stations: Juncus effuses, Polygonum pensylvanicum. Associate only at marsh meadow: Phalaris arundinacea (dominant). Associates only at Fox Creek: Salix nigra, Aster praealtus, Rosa palustris.

\section{VALERIANACEAE}

I. Leaves pinnate or deeply parted; plants perennial. Expected (Valeriana).

I. Leaves entire or dentate near the base; plants annual ........ Valerianella

\section{Valerianella}

I. Bracts (subtending the cymes) ciliate, or at least the outer ones.

2. Petals blue; fruit glabrous, its fertile locule turgid and ridged on the back ....

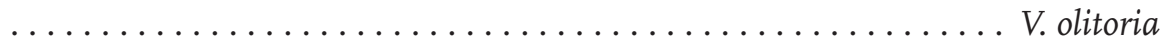

2. Petals white; fruit minutely hirsute, its fertile locule flat on the back .....

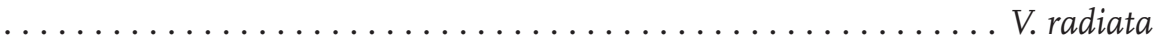

I. Bracts not ciliate. Expected (V. patellaria).

Valerianella locusta (L.) Lat.

(Valerianella olitoria (L.) Poll.)
Lewiston Cornsalad

(Corn-salad, Lamb's-lettuce)

Java Farm. Infrequent to moderately abundant along road to springhouse and in yard of old house there. Hectare 34I7. Associate species: Veronica arvensis, Duchesnea indica, Trifolium repens. Higman 607.

V. radiata (L.) Dufr.

Beaked Cornsalad

(Corn-salad Lamb's-lettuce)

Java Farm. Same location as V. olitoria above; same associate species. Higman iı26.

\section{CAMPANULACEAE}

I. Corolla regular; anthers not united into a tube; stigmas 2 or more. (Also, capsule opening by pores on the sides).

2. Corolla rotate; capsule slender-cylindric . . . . . . . . . . Specularia

2. Corolla campanulate; capsule obconic or turbinate to subglobose. Expected (Campanula).

I. Corolla irregular; anthers united into a tube; stigma I, often fringed . . . . Lobelia 


\section{Triodanis}

(Specularia)

Triodanis perfoliata (L.) Nieuwl.

(Specularia perfoliata (L.) A. DC.)

Clasping Venus' Looking-glass

(Venus' Looking-glass)

Java Farm. Infrequent along right-of-way to new entrance gate, around main buildings, and at east (drier) margin of Phalaris arundinacea meadow. Variety of associate species. Higman 723,782 .

\section{Lobelia}

I. Flowers I8-45 mm long, including the hypanthium; corolla tube with longitudinal openings near the base.

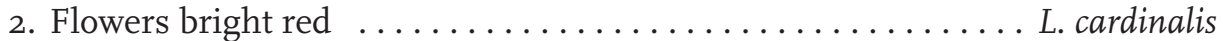

2. Flowers blue or purple. Expected (L. puberula).

I. Flowers 7-I8 mm long, including the hypanthium; corolla tube without openings.

3. Leaves linear to narrowly lanceolate. Expected (L. nuttalli).

3. Leaves oblong-lanceolate or ovate.

4. Stem long-hirsute; bracts of raceme leafy below, gradually reduced upward. (Also, flowers bluish-white) . . . . . . . . . . . . . . L. inflata

4. Stem densely short-pubescent near base; bracts of raceme all reduced from cauline leaves. Expected (L. spicata).

Lobelia cardinalis L.

Cardinalflower

(Cardinal-flower)

Java Farm. One station: On Muddy Creek at transition between forested stream valley and upstream limit of salt marsh. Hectare 3413. Associate species: Quercus falcata, Panicum virgatum, Typha latifolia, Rosa palustris, Mikania scandens, Pluchea camphorata. Higman 83.

L. inflata L.

Indian-tobacco

Ivy Neck. One station: along abandoned road through central Scaffold Peninsula. Approx. hectare 5722. Canopy of Quercus alba, Quercus velutina, Liriodendron tulipifera, Carya tomentosa. Associate species: Vernonia glauca, Amphicarpa bracteata. Higman 966.

\section{ASTERACEAE (COMPOSITAE)}

Keys to Tribes

I. Disc-flowers absent (immature ray-flowers at center of head may appear discoid); sap milky . . . . . . . . . . . . . . . . Cichorieae (p. I99)

I. Disc-flowers present, ray-flowers present or absent; sap clear. 
2. Disc-corollas deeply cleft, resembling rays (true ray-flowers absent). (Also, anthers caudate at base; plants often prickly) ........ Cynareae (p. 202)

2. Disc-corollas with small terminal lobes or truncate.

3. Receptacle with chaffy bracts. (Also, pappus of awns or chaff, or absent).

4. Outer and sometimes inner phyllaries herbaceous; style-branches usually with pointed, hirsute appendages; leaves usually opposite . . . . . . .............................. Heliantheae (p. 203)

4. All phyllaries scarious-margined; style-branches truncate, pubescent only at the tip, not appendaged; leaves alternate .... . . Anthemideae (p. 207)

3. Receptacle naked (occasionally with slender fimbrillae in Astereae (Solidago) and in Helenieae). (Also, pappus usually of capillary bristles).

5. Style-branches long and appendaged.

6. Pappus of awns, scales, or absent. (Also, style-branches usually with pointed, hirsute appendages) ........ Helenieae (p. 208, Expected)

6. Pappus of capillary bristles.

7. Ray-flowers absent; disc-flowers white or lavender; style-branches cylindrical or clavate, glabrous or nearly so.

8. Style-branches hispidulous, with pointed tips; anthers sagittate; heads in cymes or glomerules; leaves without resin dots, alternate .................... Vernonieae (p. 208)

8. Style-branches glabrous, with round or obtuse tips; anthers round-based; heads in corymbs (racemes in Liatris); leaves with resin dots, opposite or whorled (alternate in Liatris) . . . . . . .

........................ Eupatorieae (p. 209)

7. Ray-flowers present; disc flowers yellow; style-branches narrowly lanceolate, hirsute on the outside .......... Astereae (p. 2I2)

5. Style-branches short, not appendaged.

9. Phyllaries entirely herbaceous. (Also, phyllaries in only one series; style-branches truncate, pubescent only at tip; pappus bristles capillary, very soft, copious; leaves alternate (except in Arnica) . . . . . . . $\ldots \ldots \ldots \ldots \ldots \ldots \ldots \ldots$ Senecioneae (p. 22I)

9. Phyllaries partly or entirely scarious.

Io. Anthers sagittate, appendaged at base; pappus of capillary bristles; ray flowers absent in genera collected. (Also, plant usually wooly, except in Pluchea) . . . . . . . . . . . . Inuleae (p. 222)

Io. Anthers not sagittate, unappendaged; pappus a low crown or absent; ray flowers present in genera collected $\ldots \ldots \ldots \ldots \ldots$

Anthemideae (p. 207) 


\section{Cichorieae}

I. Pappus chaffy or of both chaff and bristles.

2. Flowers yellow; pappus of both chaff and bristles. Expected (Krigia).

2. Flowers blue or white; pappus of small chaffy scales ........ Cichorium

I. Pappus of many bristles.

3. Pappus plumose. Expected (Hypochoeris).

3. Pappus capillary or scabrous.

4. Achenes with short, hard points above.

5. Stems leafy; heads small, few-flowered ............ Chondrilla

5. Stems scapose; heads broad, many-flowered ........... Taraxacum

4. Achenes usually smooth (fluted and somewhat rough all over in Pyrrhopappus).

6. Achenes flat or flattish; leaves, or most of them, sharply cleft to pinnatifid. 7. Achenes tapering to acute or blunt summit, beakless; flowers 80 or more per head; leaves spiny ............... Sonchus

7. Achenes tapering to a stout, broadened summit or to a filiform beak; flowers 6 to 30 per head; leaves not spiny except in one expected

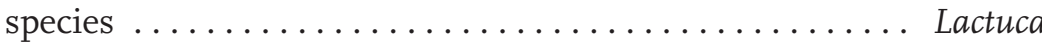

6. Achenes columnar or nearly so; leaves entire to remotely dentate or serrate, not pinnatifid.

8. Achenes with long, slender beak. (Also, involucre double, the outer phyllaries short; pappus often rust-colored but ivory-white in specimens collected) ................... Pyrrhopappus

8. Achenes beakless (but may taper to apex).

9. Phyllaries in a single series. Expected (Crepis).

9. Phyllaries in 2 or more series.

Io. Flowers white or pink; inflorescence a branching raceme or panicle of drooping heads; involucre cylindric. Expected (Prenanthes).

ıo. Flowers yellow; inflorescence a corymb or panicle of erect or ascending heads; involucre campanulate or broader . . . . . .

Hieracium

Cichorium intybus L.

\section{Cichorium}

(Cichorium intybus L., including forma alba Neum.)

Chicory

Java Farm. One station at junction of old entrance road and road to springhouse. Hectare 3408.

Ivy Neck. Infrequent at borders of cornfields on Cheston Peninsula. Also along tractor trail to artificial pond. Hectare 472I. Higman 952. 
Ivy Neck. Two stations: along trail from parking area and trail to artificial pond, above drainage to Cheston Creek. Hectare 472I. Associate species: Cichorium intybus, Mentha spicata, Ambrosia trifida, Agrostis alba. Higman I024, I218.

\section{Taraxacum}

I. Mature achenes tuberculate only above the middle.

2. Leaves dissected to midrib; achenes reddish. Expected (T. erythrospermum).

2. Leaves not dissected to midrib; achenes brownish .......... officinale

I. Mature achenes tuberculate to base ................ Tatilobum

Taraxacum officinale F.H. Wigg.

(Taraxacum officinale Weber)
Common Dandelion

(Dandelion)

Java Farm and Ivy Neck. Infrequent along roadsides, usually in unshaded locations. Higman 487, 579.

T. officinale F.H. Wigg. ssp. officinale

(T. latilobum DC. (combined with T. officinale in Britton and Brown))
Common Dandelion

(Dandelion)

Java Farm. One station: entrance gate on old Muddy Creek Road. Hectare 2463. Associate species: Taraxacum officinale, Galium aparine, Trifolium pretense, Geranium carolinianum, Commelina communis. Higman Iıзі.

\section{Sonchus}

Sonchus asper (L.) Hill

Spiny Sowthistle

(Sow-thistle)

Java Farm. One station: base of tile silo at main building area. Hectare 2590. Higman 827 .

\section{Lactuca}

I. Achenes tapering to a stout, broadened summit; flowers blue.

2. Pappus tawny or olive. Expected (L. biennis).

2. Pappus white ....................... floridana

I. Achenes tapering to a filiform beak; flowers yellow.

3. Heads 5- to 8-flowered; achenes 5- to 7-ribbed on each face, oblanceolate to narrowly obovate. Expected (L. scariola, L. saligna).

3. Heads I2- to 20-flowered; achenes I- to 3-ribbed on each face, oblong, elliptic, or oval. (Also, beak jointed) $\ldots \ldots \ldots \ldots \ldots \ldots \ldots \ldots$. canadensis 
Java Farm. One station: along old entrance road between gate at old Muddy Creek Road and culvert of Muddy Creek. Canopy of Fagus grandifolia, Quercus alba, Liriodendron tulipifera, Carya tomentosa, etc. Associate species: Eupatorium purpureum, Agrimonia microcarpa, Desmodium paniculatum, D. glutinosum, D. glabellum, Elephantopus carolinianus. Higman 30.

L. canadensis L.

Canada Lettuce

(Lettuce)

Java Farm. One station in old field. Hectare 3504. Associate species: Solidago rugosa, S. altissima, Aster lateriflorus. Higman 33.

Pyrrhopappus carolinianus (Walter) DC.

Pyrrhopappus

(Pyrrhopappus carolinianus (Walt.) DC.)

Carolina Desert-chicory

(False Dandelion)

Ivy Neck. One station: tractor trail to abandoned field on Scaffold Peninsula. Hectare 4740. Associate species: Anagallis arvensis, Galinsoga ciliate, Rumex crispus. Higman 957.

\section{Hieracium}

I. Involucre barely Imbricated; phyllaries equal. (Also, stem scapose or cauline leaves few; rhizome present). Expected (H. pratense, $H$. florentinum).

I. Involucre clearly imbricated; phyllaries unequal.

2. Branches of inflorescence with basal bracts; inflorescence a panicle or corymb.

3. Leaves chiefly basal at anthesis, broader than the scattered or absent cauline ones; bracts minute. (Also, tips of rays fringe-cut) . . . . H. venosum

3. Leaves extending regularly up the stem; bracts leafy.

4. Stem glabrous except at villous base; branches of inflorescence glabrous. Expected (H. paniculatum).

4. Stem and branches of inflorescence densely pubescent. (Also, panicle may be short-cylindric; peduncles densely glandular) ...... H. scabrum

2. Branches of inflorescence without bracts; inflores- a cylindric panicle. Expected (H. gronovii). 
Java Farm and Ivy Neck. Infrequent, chiefly along estuary shores under canopy of Quercus prinus, Quercus alba, Carya tomentosa, etc. Associate species: Gerardia virginica, Gaylussacia baccata, Vaccinium vacillans, Epigaea repens. Also found along bank of tidal portion of Muddy Creek, under canopy of Fagus grandifolia and Quercus spp. Hectare 355I. Shrub layer at this point resembles that under Quercus prinus. Higman 655,842 .

H. scabrum Michx.

Rough Hawkweed

(Hawkweed)

Java Farm. Two stations: one on south-facing slope over abandoned pasture. Hectare 2458. Scattered Liquidambar styraciflua, Liriodendron tulipifera, and Pyrus malus. Spreading ground cover of Lonicera japonica, also Rubus spp. and Rhus radicans. Second station at old entrance gate. Hectare 2463. Edge of canopy of Fagus grandifolia, Quercus spp., Carya tomentosa, Liriodendron tulipifera, etc. Associate species there: Geranium carolinianum, Galium aparine, Commelina communis. Higman I9I, 353 .

\section{Cynareae}

I. Involucre not spiny; flowers blue, the marginal ones resembling rays . . . . .

I. Involucre spiny; flowers pink or red-violet, all alike.

2. Leaves not spiny, the lower ones cordate; phyllaries spiny, with hooked tips

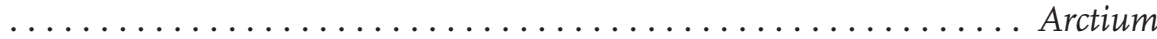

2. Leaves spiny, narrow at base; phyllaries spiny, with straight tips .... Cirsium

\section{Centaurea}

Centaurea cyanus L.

Garden Cornflower

(Cornflower, Bachelor's Button)

Ivy Neck. One station: tractor trail to abandoned field on Scaffold Peninsula. Hectare 4740. Associate species: Anagallis arvensis, Galinsoga ciliata, Pyrrhopappus carolinianus, Taraxacum officinale, Rumex crispus. Higman 808.

\section{Arctium}

Arctium minus Bernh.

Lesser Burdock

(Arctium minus (Hill.) Bernh.)

(Common Burdock)

(A. minus Schk.)

Java Farm. One station: rubbish pile at main building area. Hectare 2580. Associate species: Eleusine indica, Datura stramonium, Solanum nigrum, Amaranthus spinosus, Phytolacca americana. Higman 6. 
I. Flowers perfect.

\section{Cirsium}

2. Upper half of stem and branches with long, prickly wings decurrent from the leaves .............................. vulgare

2. Upper half of stem and branches not long-winged.

3. Leaves green beneath. Expected (C. muticum).

3. Leaves with dense white felt beneath.

4. Heads subtended by spiny leafy bracts; peduncles short, leafy.

5. Principal cauline leaves deeply pinnatifid into linear-lanceolate lobes

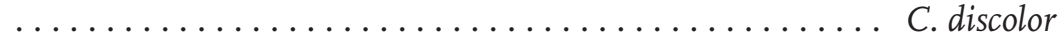

5. Principal cauline leaves entire or shallowly lobed. Expected (C. altissimum).

4. Heads not subtended by leafy bracts; peduncles long, with scattered and reduced bracts. Expected (C. virginianum).

I. Flowers dioecious. (Also, leaves green both sides)

C. arvense

Cirsium vulgare (Savi) Ten.

Bull Thistle

(Cirsium vulgare (Savi) Tenore)

Ivy Neck. One station: edge of cornfield on Cheston Peninsula, near head of Cheston Creek estuary. Hectare 4755. Associate species: Datura stramonium, Abutilon theophrasti. Higman 1220.

C. discolor (Muhl. ex Willd.) Spreng.

Field Thistle

(C. discolor (Muhl.) Spreng.)

(Thistle)

Java Farm. Moderately abundant along old entrance road south of main building area, and in grassy parts of old fields. Hectares 2590, 3500, 3504. Associate species: Poa pratensis (dense turf), Asclepias syriaca, Apocynum cannabinum, Erigeron canadensis, Aster dumosus. Higman 44, 337.

C. arvense (L.) Scop.

Canada thistle

(Canadian Thistle)

Java Farm. One station: beside old entrance road just south of main building area. Hectare 3500. Associate species: Cirsium discolor, Asclepias syriaca, Verbascum thapsus, V. blattaris. Higman 888.

\section{Heliantheae}

I. Ray flowers absent.

2. Shrub; salt marsh habitat; heads monoecious, the pistillate flowers I to 5, marginal, without corolla; the staminate flowers numerous, central, with

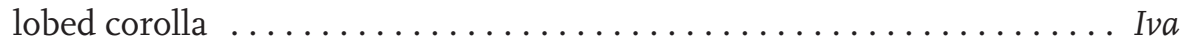


2. Herbs; terrestrial habitat; heads dioecious, the pistillate ones clustered below spikes of staminate ones; pistillate heads I- or 2-flowered; staminate heads 5- to 20 -flowered.

3. Sandy beach habitat; phyllaries of staminate involucre not united; fruiting head a very prickly bur, 2 -seeded; leaves simple . . . . . . . Xanthium

3. Roadside and field habitat; phyllaries of staminate involucre united; fruiting head not burlike, I-seeded; leaves lobed or dissected. (Also, staminate heads inverted) $\ldots \ldots \ldots \ldots \ldots \ldots \ldots \ldots \ldots \ldots \ldots \ldots \ldots$. . . . . . . .

I Ray flowers present.

4. Disc flowers sterile; ray flowers fertile. Expected (Polymnia, Silphium, Parthenium).

4. Disc flowers fertile; ray flowers fertile or sterile.

5. Leaves decurrent down the stem.

6. Ray flowers sterile; phyllaries bent downward, few; leaves pinnately veined; flowers pale yellow ................... Actinomeris

6. Ray flowers pistillate; phyllaries imbricated, many; leaves 3-nerved; flowers golden-yellow ................. Verbesina

5. Leaves not decurrent.

7. Achenes turbinate, 5-angled. (Also, leaves opposite; rays white; disc

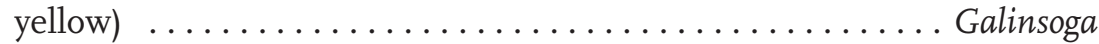

7. Achenes flat, 4-angled or, if 5-angled, subterete and slender.

8. Receptacle conical to columnar; leaves alternate. (Also, rays orange;

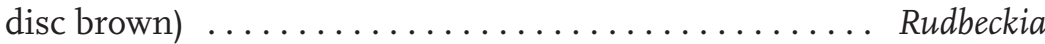

8. Receptacle flat or slightly convex; leaves opposite in all genera collected or expected.

9. Flowers white; chaff of receptacle bristly; pappus a minute bristly

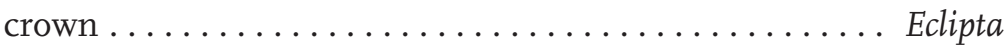

9. Flowers yellow; chaff of receptacle broader, flattish; pappus of teeth, deciduous scales, or wanting.

Io. Pappus of retrorsely or antrorsely barbed awns or teeth (barbs not always apparent on immature flowers). (Also, achenes ciliate along the margins) $\ldots \ldots \ldots \ldots \ldots \ldots$ Bidens

Io. Pappus of unbarbed teeth, of scales, or wanting. Expected (Heliopsis, Coreopsis).

Iva

Iva frutescens $\mathrm{L}$.

Jesuit's bark

(Marsh-elder, Highwater-shrub)

Java Farm and Ivy Neck. Moderately abundant in salt marshes, especially the more inland parts. Associate species: Distichlis spicata, Solidago sempervirens, Aster subulatus, Spartina patens, Hibiscus palustris. Higman 53, II4. 
Ivy Neck. One station: sandbar of cut-off pond on Rhode River shore of Cheston Peninsula. Hectare 5800. Associate species: Spartina alterniflora, Asparagus officinalis, Strophostyles helvola. Higman rozo.

\section{Ambrosia}

I. Leaves entire or palmately cleft, opposite; staminate involucres unilaterally 3-ribbed; fruits 3 to $\mathrm{I} 2 \mathrm{~mm}$ long $\ldots \ldots \ldots \ldots \ldots \ldots \ldots \ldots \ldots \ldots \ldots \ldots \ldots$ trifida

I. Leaves pinnately lobed to bi- or tri-pinnatifid, both opposite and alternate; staminate involucres obscurely radiate-veined; fruit smaller than above . . . . . . . . .

$$
\text { A. artemisifolia }
$$

Ambrosia trifida L.

Great Ragweed

(Giant Ragweed Buffalo Weed)

Java Farm and Ivy Neck. Infrequent to moderately abundant along unshaded or partly shaded roads, tractor trails, and field margins. Wide variety of associate species. Higman 252, 3II, IO25.

A. artemisiifolia L.

Annual Ragweed

(Common Ragweed, Hog Weed)

Java Farm and Ivy Neck. Infrequent to moderately abundant along unshaded or partly shaded roads and field margins. Wide variety of associate species. Higman iı67.

\section{Verbesina}

(Actinomeris)

\section{Verbesina alternifolia (L.) Britton ex Kearney}

Wingstem

(Actinomeris alternifolia (L.) DC.)

(Verbesina alternifolia (L.) Britt.)

(Wing-stem)

Java Farm. One station: source of stream which runs under new entrance road at junction with road to pier. Hectare 2599. Canopy of Quercus velutina and Juglans nigra. Associate species: Impatiens capensis. Higman 228.

Verbesina occidentalis (L.) Walter

Verbesina

(Verbesina occidentalis (L.) Walt.)

Yellow Crownbeard

(Crown-beard)

Java Farm. Moderately abundant in moist, partly shaded areas, especially forest glades and forest margins of fields. Most abundant in hectares 2479, 2561, 3429, and 3508. Often in virtually pure stands. Higman I47. 
Ivy Neck. Infrequent along tractor trails and roadsides. Hectare 4740. Also along trail to artificial pond on Murray farm. Associate species: Perilla frutescens, Taraxacum officinale, Daucus carota. See also Appendix II. Higman 836, Io6o.

\title{
Rudbeckia hirta L. var. pulcherrima Farw.
}

\section{Rudbeckia}

(Rudbeckia serotina Nutt.)

\author{
Blackeyed Susan \\ (Black-eyed Susan)
}

Ivy Neck. Infrequent to moderately abundant in abandoned field on Scaffold Peninsula. Hectares 4762, 4772. Wide variety of associate species. Also one station beside cultivated field on Cheston Peninsula. Higman 8I6, i242.

\section{Eclipta prostrata (L.) L.}

\section{Eclipta}

(Eclipta alba (L.) Hassk.)

False Daisy

Java Farm. One station: growing in rotted stump beside tidal portion of Muddy Creek, near upstream limit of salt marsh vegetation. Hectare 34I3. Accessible only by boat at high tide. Associate species: Pluchea camphorata, Scirpus validus, Smilax rotundifolia, Typha latifolia. Higman 258.

\section{Bidens}

I. Primary leaves bi- or tri-pinnate; achenes linear, very unequal, the inner 2 to 3 times as long as the outer and inner involucre ............. B. bipinnata

I. Primary leaves once-pinnate or simple; achenes broadened from base to sum$\mathrm{mit}$, the inner not over $\mathrm{I} / 3$ longer than the inner involucre.

2. Primary leaves simple or with 3 to 5 coarse lobes, without distinct leaflets.

3. Achenes with a convex cartilaginous summit. Expected (B. laevis, B. cernua).

3. Achenes truncate or concave at summit, not cartilaginous.

4. Mature central achenes strongly 4-angled. (Also, leaves undivided or with divergent basal lobes; phyllaries ascending or loosely spreading)

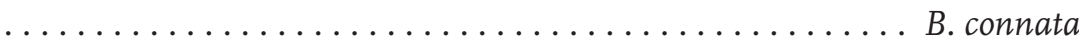

4. Mature central achenes flattish, with slender or obscure midribs. Expected (B. comosa).

2. Primary leaves deeply pinnate-divided or with distinct leaflets.

5. Rays wanting or shorter than outer involucre; leaflets uncleft. Expected (B. frondosa).

5. Rays showy, conspicuously longer than outer involucre; leaflets pinnately incised or coarsely serrate. 
6. Outer involucre of 8 to I2 smooth or finely ciliate spreading, acute or blunt phyllaries 4 to $7 \mathrm{~mm}$ long. Expected (B. aristosa).

6 . Outer involucre of $\mathrm{I} 2$ to 25 puckered or coarsely serrate ciliate spreading or reflexed-curling acuminate phyllaries 7 to $20 \mathrm{~mm}$ long.... B. polylepis

Bidens bipinnata L.

Spanish Needles

Java Farm. Infrequent to moderately abundant along roadsides and in cracked pavement at main building area. Associate species: Juncus tenuis, Aster dumosus, Perilla frutescens, Lamium amplexicaule, Melilotus alba. Higman 77, 357.

B. connata Muhl. ex Willd.

(B. connata Muhl. var. petiolata (Nutt.) Farw.)

(B. tripartita L.)

Purplestem Beggarticks

(Beggar-ticks, Stick-tight)

Java Farm. One station: sand bar in bed of Muddy Creek, upstream from culvert of old entrance road. Hectare 2467. Canopy of Fagus grandifolia, Platanus occidentalis, Quercus alba; understory of Lindera benzoin. Associate species: Impatiens capensis. Higman 168.

\section{B. aristosa (Michx.) Britton \\ (B. polylepis Blake)}

Bearded Beggarticks

(Beggar-ticks, Stick-tight)

Java Farm. One station: forest margin of old field east of road to springhouse. Hectare 3428. Adjacent canopy of Fagus grandifolia and Quercus velutina. Associate species: Verbesina occidentalis. Higman I48.

\section{Anthemideae}

I. Receptacle chaffy, at least toward summit or center.

2. Heads numerous in close corymbs; achenes oblong, flattened; receptacle flattish (prolonged in fruit) $\ldots \ldots \ldots \ldots \ldots \ldots \ldots \ldots \ldots \ldots$ Achillea

2. Heads solitary at tips of branches and branchlets; achenes terete, angled, or ribbed; receptacle conical ....................... Anthemis

I. Receptacle naked. (Also, disc flowers yellow).

3. Heads in spikes, racemes, or panicles. (Also, rays and pappus absent). Expected (Artemisia).

3. Heads solitary or in corymbs.

4. Receptacle conical, at least in fruit. (Also, rays and pappus absent). Expected (Matricaria).

4. Receptacle flattish or low-convex.

5. Ray flowers present; pappus none; achenes 5- to Io-nerved. (Also, rays white (in species collected) or yellow)............ Chrysanthemum

5. Ray flowers absent; pappus a low crown; achenes 3- to 5-angled. Expected (Tanacetum). 
Java Farm. Infrequent to moderately abundant at main building area, in grassy fields south of main buildings (hectares 2499, 3409), and in triangular area at entrance gate on old Muddy Creek Road (hectare 2463). Associate species: Poa pratensis (turf), Erigeron canadensis, Plantago lanceolata, Duchesnea indica, Cyperus strigosus. Higman IO, $5 \mathrm{I} 7$.

\section{Anthemis}

\section{Anthemis arvensis L.}

Corn Chamomile

(Anthemis arvensis L., var. agrestis (Wallr.) DC.)

(variety not in Britton and Brown)

(Corn-chamomile)

Ivy Neck. One station: beside tractor trail on Scaffold Peninsula, between cornfield and grassy abandoned field. Hectare 477I. Associate species: Hordeum pusillum, Plantago virginica, Linaria canadensis, Specularia perfoliata. Higman 694.

Leucanthemum vulgare Lam.
(Chrysanthemum leucanthemum L.)

\section{Leucanthemum}

(Chrysanthemum)

Java Farm. Infrequent in old fields. Dominant species: Rhus radicans, Rubus spp., Solidago spp., Lonicera japonica. Associate species: Oxalis stricta, Rumex acetosella, Lespedeza cuneata, Barbarea vulgaris. Higman 652, 758.

\section{Helenieae}

No species collected from this tribe; Helenium spp. Expected.

\section{Vernonieae}

I. Heads not in glomerules, several-flowered; phyllaries uniform, imbricated; corolla regularly 5-cleft; pappus double; leaves not rosulate . . . . . . . Vernonia

I. Heads in glomerules, 2-5 flowered; phyllaries alternately flat and folded; corolla slightly irregular; pappus single; basal leaves rosulate ........ Elephantopus

\section{Vernonia}

I. Phyllaries long-acuminate or with a filiform appendage.

2. Pappus purplish; phyllaries ovate below appendage, with broad scarious

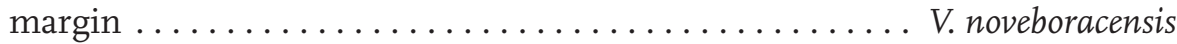

2. Pappus creamy; phyllaries lanceolate below appendage, with slightly scarious

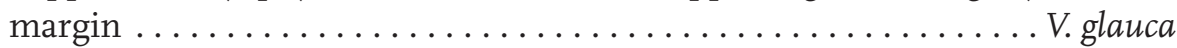

I. Phyllaries obtuse or acute. Expected (V. altissima). 
Java Farm. Infrequent: one station at north margin of freshwater part of Fox Creek marsh (hectare 3515); one station in old field south of old entrance road and east of road to springhouse (hectare 34I8); one station near Muddy Creek, at upstream limit of salt marsh vegetation (hectare 3413). Higman 84, I29, 484.

V. glauca (L.) Willd.

Broadleaf Ironweed

(Ironweed)

Ivy Neck. Infrequent: along abandoned road through central Scaffold Peninsula. Hectare 5732. Canopy of Quercus alba, Liriodendron tulipifera, Quercus velutina, Carya tomentosa. Associate species: Amphicarpa bracteata, Lobelia inflate, Gerardia virginica. Higman 965.

Elephantopus carolinianus Raeusch.

\section{Elephantopus}

(Elephantopus carolinianus Willd.)

Carolina Elephantsfoot

(Elephant's-foot)

Java Farm. Infrequent to moderately abundant in moist, shaded areas, especially near streams; grows under a variety of dense, mixed hardwood canopies. Associate species vary, include: Panicum Clandestinum, Aster vimineus, Perilla frutescens, Muhlenbergia schreberi, Asplenium platyneuron, Polystichum acrostichoides, Aster acuminatus, Glyceria striata, Geum canadense, Onoclea sensibilis. Higman 37, I83, 236, 306, 47I, 1003.

\section{Eupatorieae}

I. Leaves alternate; heads in racemes or spikes (Also, flowers rose-purple) ... Liatris

I. Leaves opposite; heads in corymbs.

2. Twining vine; phyllaries 4 ; flowers $4 \ldots \ldots \ldots \ldots$ Mikania

2. Erect, not twining; phyllaries more than 4 ; flowers $3-40 \ldots \ldots$ Eupatorium

\section{Liatris}

Liatris pilosa (Aiton) Willd.

(Liatris graminifolia (Walt.) Willd.)
Shaggy Blazing Star

(Button-snakeroot, Blazing-star)

Java Farm. One station: Fox Point. Hectare 3558. Canopy of Quercus prinus, Quercus alba. Associate species: Gerardia virginica, Epigaea repens, Gaylussacia baccata. Higman 430. 
Java Farm. Infrequent to moderately abundant in moist habitats, fresh or slightly saline. Moderately abundant near landward borders of salt marshes; associate species there: Iva frutescens, Pluchea camphorata, Polygonum hydropiperoides, Panicum clandestinum, Hibiscus palustris, Kosteletzkya virginica.

Also moderately abundant in marsh at west corner of Farm, hectare 2470. Associate species there: Scutellaris lateriflora. Infrequent in old fields, hectares 2580, 34I8. Associate species there: Eupatorium serotinum, Solidago altissima, Aster novibelgii. Higman 81, I46, I96, 408.

\section{Conoclinium, Eutrochium, Eupatorium}

I. Receptacle conical; base of stem with prostrate superficial stolons; flowers blueviolet $\ldots \ldots \ldots \ldots \ldots \ldots \ldots \ldots \ldots \ldots \ldots \ldots \ldots \ldots \ldots \ldots \ldots \ldots \ldots$ coelestinum

I. Receptacle flat; base of stem without superficial stolons; flowers white to purple. 2. Involucre cylindric, the outer phyllaries closely imbricated, purple to creamy white. (Also, leaves usually whorled in 3's to 5's, lanceolate to ovate).

3. Inflorescence round-topped; lower leaf surfaces and upper stem with glutinous dots (sometimes absent in E. purpureum).

4. Leaves palmately 3-veined; involucre and flowers purple. Expected (E. dubium).

4. Leaves pinnately veined; involucre and flowers white to pale lavender. (Also, stem with dark purple nodes; fresh plant strongly vanillascented $\ldots \ldots \ldots \ldots \ldots \ldots \ldots \ldots \ldots \ldots \ldots \ldots \ldots \ldots \ldots$. purpureum

3. Inflorescence flat-topped; lower leaf surfaces and upper stem not glutinousdotted. (Also, stem deep purple or purple-spotted; florets 8-20). Expected (E. maculatum).

2. Involucre lax, the outer phyllaries not closely imbricated, green. (Also, leaves alternate or opposite, whorled only in E. hyssopifolium, and then linear to narrowly lanceolate).

5. Leaves sessile or nearly so.

6. Heads with io to 40 flowers.

7. Leaves perfoliate, lanceolate, acuminate ........ E. perfoliatum

7. Leaves with truncate or round bases, ovate, acute to blunt. Expected (E. aromaticum).

6. Heads with 5 to 7 flowers.

8. Leaves broadly subtruncate, round or cordate at base. Expected (E. album).

8. Leaves tapering to base.

9. Phyllaries with conspicuously scarious tips, the inner ones equaling the flowers. Expected (E. album). 
9. Phyllaries without conspicuously scarious tips, shorter than the flowers. (Also, leaves linear and entire to lanceolate and serrate, all but the lowest with dense axillary fascicles, leaves often in 3's) $\ldots \ldots \ldots \ldots \ldots \ldots \ldots \ldots \ldots \ldots \ldots$. hyssopifolium

5. Leaves definitely petioled. (Also, heads with 8 to 40 flowers).

Io. Outer phyllaries oblong, densely pubescent, not mucronate-tipped; phyllaries in 2 or 3 lengths . ............... serotinum

Io. Outer phyllaries acute or blunt, glabrous, mucronate-tipped; phyllaries equal in length $\ldots \ldots \ldots \ldots \ldots \ldots \ldots \ldots \ldots \ldots \ldots$. rugosum

\section{Conoclinium}

Conoclinium coelestinum (L.) DC.

Blue Mistflower

(Eupatorium coelestinum L.)

(Mistflower, Blue Boneset)

Java Farm. One station: abandoned road along north border between Muddy Creek and entrance gate at Muddy Creek Road. Hectare 2465. Canopy of Fagus grandifolia, Quercus alba, C. velutina, Liriodendron tulipifera. Associate species: Agrimonia rostellata, Houstonia purpurea.

Ivy Neck. One station: west fork of Cheston Creek drainage, adjacent abandoned field on Scaffold Peninsula. Hectare 4752. Canopy of Platanus occidentalis, Diospyros virginiana, Liquidambar styraciflua. Associate species: Duchesnea indica. Higman I057, 1086.

\section{Eutrochium}

Eutrochium purpureum (L.) E.E. Lamont

(Eupatorium purpureum L.)
Sweetscented Joe Pye Weed

(Sweet Joe Pye-weed)

Java Farm. One station: along old entrance road between Muddy Creek and gate at Muddy Creek Road. Canopy of mature Fagus grandifolia, Quercus alba, Liriodendron tulipifera, etc. Associate species: Lactuca floridana, Agrimonia microcarpa, Desmodium glutinosum, Elephantopus carolinianus. Higman 3I.

\section{Eupatorium, Ageratina}

Eupatorium perfoliatum L.

Common Boneset

(Thoroughwort, Boneset)

Java Farm. One station: bottom of Muddy Creek valley, approx. hectare 2486. Canopy of Fraxinus americana, Platanus occidentalis; understory of Lindera benzoin.

Ivy Neck. One station: moist area along abandoned road on Cheston Peninsula. Approx. hectare 4799. Canopy of Juglans nigra, Pinus taeda, P. virginiana, Liquidambar styraciflua. Higman 68. 
Java Farm. One station in old field north of Fox Point Road; hectare 350I. Also, one station at south margin of old field between Fox Point Road and Hog Island salt marsh; hectare 3585. Dominant species at both stations: Rhus radicans, Lonicera japonica, Solidago altissima. Rubus spp. Higman I36, 423.

\section{E. serotinum Michx.}

\section{Lateflowering Thoroughwort}

(Thoroughwort)

Java Farm. Infrequent to moderately abundant in old fields; dominant species: Rhus radicans, Lonicera japonica, Solidago altissima, Rubus spp. Also one station on Muddy Creek at upstream limit of salt marsh vegetation, and one beside new entrance road at the culvert of Fox Creek drainage. Higman 79, I27, 315, 338, I0I7, 1067.

\section{Ageratina altissima (L.) R.M. King \& H. Rob. var. altissima}

White Snakeroot (E. rugosum Houtt., forma villicaule Fern.)

Java Farm. One station: old fields south of main buildings, near old entrance road. Hectares 3408, 3409. Poa pratensis turf being invaded by dense Rhus radicans, Lonicera japonica, and Campsis radicans. Higman 488.

\section{Astereae}

I. Shrubs; ray flowers absent; disc flowers dioecious . . . . . . . . . . Baccharis

I. Herbs; ray flowers present (very small in a few species); disc flowers perfect.

2. Ray flowers yellow (white in Solidago bicolor, the rays then small, ascending; heads small, in a dense panicle).

3. Pappus of disc flowers either of chaffy awns or of double bristles (long inner bristles, short outer ones). Expected (Chrysopsis, Grindelia).

3. Pappus of disc flowers a single series of slender bristles ...... Solidago

2. Ray flowers white, pink, purple, or blue.

4. Pappus none or very short. (Also, rays pistillate, lilac; aspect resembling Aster). Expected (Boltonia).

4. Pappus of many long slender bristles.

5. Involucre slenderly cylindric or ovoid; disc flowers pale yellow to white; achenes very silky. (Also, phyllaries appressed, cartilaginous, whitish, with short, spreading green tips; leaves ciliate) ......... Sericocarpus

5. Involucre saucer-shaped or hemispherical to campanulate; disc flowers yellow or sometimes purplish; achenes glabrous or pubescent, not silky. 6. Phyllaries definitely imbricated, the outer series as long as the inner Aster

6. Phyllaries not imbricated or very slightly so, in one series or with a very short outer one $\ldots \ldots \ldots \ldots \ldots \ldots \ldots \ldots$ Erigeron 


\section{Baccharis}

Baccharis halimifolia L.

Eastern Baccharis

(Sea-myrtle)

Java Farm and Ivy Neck. Abundant to moderately abundant in salt marshes, especially the wetter areas near tidal channels. Associate species: Spartina alterniflora, S. cynosuroides. Often parasitized by Cuscuta gronovii.

Other habitats: Java Farm. One station in old field, near tributary of Fox Creek drainage. Hectare 3503. Dense Rubus spp., Lonicera japonica, and Rhus radicans.

Ivy Neck. One station on sandy beach of Rhode River, on Murray property. Hectare 4704. Associate species: Amorpha fruticosa. Higman 215, 253, 362.

\section{Euthamia, Solidago}

I. Inflorescence a flat-topped corymb, the heads not racemosely arranged on the branches.

2. Leaves not glandular-punctate, the basal petioled and usually much larger than the upper; heads pedicelled; ray-flowers 6 to Io, fewer than the discflowers; receptacle naked. Expected (S. rigida).

2. Leaves glandular-punctate, all sessile and similar in size; heads sessile in small glomerules; ray-flowers 6 to 20, more than the disc-flowers; receptacle

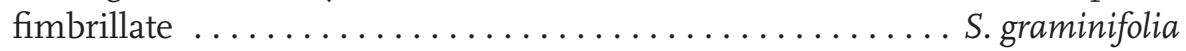

I. Inflorescence of axillary clusters or a terminal panicle.

3. Inflorescence of axillary clusters. (Also, basal leaves small and soon deciduous, not forming rosettes; achenes hairy).

4. Stem terete, glaucous when fresh; primary leaves lanceolate to narrowly

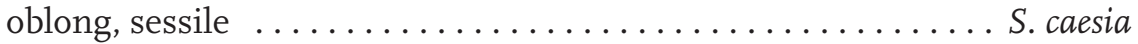

4. Stem angled, not glaucous; leaves ovate, contracted to a winged petiole. Expected ( $S$. flexicaulis).

3. Inflorescence a terminal panicle.

5. Branches of panicle not recurved, panicle not nodding at summit; heads spirally arranged on the branches, not strongly I-sided.

6. Cauline leaves gradually decreasing in size upward; basal rosettes wanting. Expected (S. puberula, S. speciosa).

6. Cauline leaves sharply decreasing in size upward; basal rosettes present, of large leaves.

7. Tips of phyllaries strongly recurving. Expected (S. squarrosa).

7. Tips of phyllaries appressed-ascending or slightly spreading.

8. Leaves pilose or bristly on one or both surfaces.

9. Rays silvery white; outer phyllaries with green tips and contrasting whitish bases and margins ......... S. bicolor

9. Rays yellow; outer phyllaries with less contrast ..... S. hispida

8. Leaves glabrous or minutely puberulent. Expected (S. puberula, S. erecta). 
5. Branches of panicle recurved, panicle nodding at summit; heads borne on the upper side of the branches.

Io. Salt marsh habitat $\ldots \ldots \ldots \ldots \ldots \ldots \ldots \ldots$. S. sempervirens

Io. Terrestrial habitat.

II. Cauline leaves rapidly decreasing in size upward; basal rosettes usually present; cauline leaves 5 to 40 .

I2. Leaves sharply serrate; petioles winged, ciliate; plant otherwise glabrous or scabrous ............... S. juncea

I2. Leaves crenate to subentire; petioles wingless; stems and leaves with dense grayish puberulence $\ldots . \ldots$. S. nemoralis

II. Cauline leaves very gradually decreasing in size upward; basal rosettes absent; cauline leaves 20 to over Io०.

I3. Leaves pinnately nerved, without 2 conspicuously prolonged lateral nerves.

I4. Stem glabrous or nearly so, or puberulent only in lines. Expected (S. odora, S. elliottii).

I4. Stem villous or scabrous-pubescent throughout. (Also, conspicuous leafy bracts present on branches of panicle) ............................. S. rugosa

I3. Leaves with 2 conspicuously prolonged lateral nerves, plus the midrib.

I5. Summit of stem below panicle glabrous or sparsely pilose. Expected (S. gigantea).

I5. Summit of stem below panicle densely pilose, most of stem closely puberulent.

I6. Leaves sharply serrate; involucres 2 to $2.8 \mathrm{~mm}$ high; phyllaries slenderly tapering. Expected (S. canadensis).

I6. Leaves remotely serrate or subentire; involucres 3 to $5 \mathrm{~mm}$ high; phyllaries linear, blunt-tipped ....... ........................ S. altissima

Euthamia graminifolia (L.) Nutt. var. graminifolia

(Solidago graminifolia (L.) Salisb.)

Flat-top goldentop

(Flat-topped Goldenrod)

Java Farm. Moderately abundant in old fields. Dominant species: Rhus radicans, Campsis radicans, Lonicera japonica, Solidago altissima, Rubus spp. Scattered young mixed hardwoods.

Also, one station in freshwater part of Fox Creek marsh. Hectare 3516. Associate species: Aster puniceus, Leersia oryzoides. Higman I25, 245, 384. 
Java Farm. Infrequent along old Muddy Creek Road and around entrance gate there. Hectare 2463. Canopy of mature Fagus grandifolia, Liriodendron tulipifera. Higman $385,406$.

S. bicolor L.

White Goldenrod

Ivy Neck. One station: west shore of Cheston Creek estuary. Hectare 5725. Canopy of Quercus prinus, Q. alba, Carya tomentosa, Acer rubrum, Fagus grandifolia. Higman Io68.

S. hispida Muhl. ex Willd.

Hairy Goldenrod

(S. hispida Muhl.)

(Goldenrod)

Java Farm. One station: Fox Point. Hectare 3558. Canopy of Quercus prinus, Q. alba. Associate species: Liatris graminifolia, Epigaea repens, Gaylussacia baccata. Higman II33.

S. sempervirens L.

Seaside Goldenrod

Java Farm. Infrequent in salt marshes. Associate species: Spartina patens, Distichlis spicata, Baccharis halimifolia. Higman 213.

S. juncea Aiton

Early Goldenrod

(S. juncea Ait.)

Java Farm. One station: stand of young Pinus virginiana in old field. Hectare 3523. Associate species: Trichostema setaceum. Higman 38I.

S. nemoralis Aiton

Gray Goldenrod

(S. nemoralis Ait.)

(Goldenrod)

Java Farm. One station: old field north of Fox Point Road. Hectare 3523. Partial canopy of Pinus virginiana, Liquidambar styraciflua. Dense Lonicera japonica and Rhus radicans. Associate species: Solidago juncea, Lespedeza cuneata, Rosa palustris, R. micrantha. Higman 373.

S. rugosa Mill.

Wrinkleleaf Goldenrod

(S. rugosa Ait.)

(Goldenrod)

Java Farm. Infrequent to moderately abundant in old fields. Dominant species: Rhus radicans, Lonicera japonica, Rubus spp., Solidago altissima. Associate species: Lespedeza cuneata, Aster dumosus, A. vimineus, Eupatorium serotinum. Scattered trees: Liquidambar styraciflua, Cornus florida, Sassafras albidum. Higman 330, 349, 437. 
Java Farm. Abundant to moderately abundant in old fields. Often co-dominant with Rhus radicans, Lonicera japonica, and Rubus spp. Associate species: same as for Solidago rugosa and S. graminifolia above. Higman I42, 200, 350.

\section{Sericocarpus}

Sericocarpus asteroides (L.) Britton, Sterns \& Poggenb. Toothed Whitetop Aster (Sericocarpus asteroides (L.) B. S. P.)

(White-topped Aster)

Java Farm. One station: Fox Point. Hectare 3558. Canopy of Quercus alba, Quercus prinus. Associate species: Baptisia tinctoria, Gaylussacia baccata, Vaccinium vacillans, Kalmia latifolia, Hieracium venosum, Deschampsia flexuosa, Liatris graminifolia. Higman 436, II78.

\section{Symphyotrichum, Oclemena}

(Aster)

I. Salt marsh habitat.

2. Phyllaries ovate-acuminate, with broadly scarious margins; rays conspicuous, pale purple; stolons present ................ tenuifolius

2. Phyllaries linear, with very narrowly scarious margins; rays inconspicuous, purple; stolons absent $\ldots \ldots \ldots \ldots \ldots \ldots \ldots \ldots \ldots \ldots$. subulatus

I. Terrestrial or freshwater marsh habitat.

3. Phyllaries without herbaceous tips, strongly scarious. (Also, leaves oblonglanceolate to oblong-oblanceolate, serrate above the middle, long-acuminate)

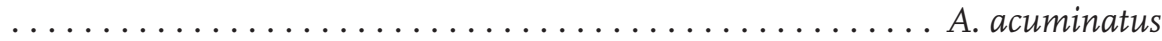

3. Phyllaries with herbaceous tips, their margins always partly herbaceous.

4. At least the basal leaves cordate and petioled. Expected (A. cordifolius, A. sagittifolius).

4. None of the leaves both cordate and petioled.

5. Bases of cauline leaves auriculate-clasping.

6. Involucre glandular-hairy. Expected (A. patens, A. novae-angliae).

6. Involucre not glandular.

7. Leaves either tapering to an abruptly widened base or glaucous and leathery. Expected (A. prenanthoides, A. laevis).

7. Leaves tapering to a narrowly auriculate base, neither glaucous nor leathery. (Also, plant up to $2.5 \mathrm{~m}$ tall; stem and branches of inflorescence hirsute or villous; phyllaries linear-attenuate, with scarious margins) ................... Auniceus

5. Bases of cauline leaves attenuate or rounded, not auriculate-clasping.

8. Leaves whitened or silvery-silky on both sides. Expected (A. concolor).

8. Leaves green. 
9. Phyllaries very hard and stiff except at their herbaceous tips. (Also, phyllaries and upper leaves subulate-tipped; upper leaves with axillary fascicles; stem pilose) .............. A. pilosus

9. Phyllaries pliable throughout.

Io. Phyllaries narrowly herbaceous, with strongly recurving tips; rays blue-violet ................. novi-belgii

Io. Phyllaries scarious-margined, the midribs herbaceous; rays white (except in A. praealtus).

II. Branches of stem widely spreading (arched-ascending in A. lateriflorus); phyllaries appressed-ascending, with herbaceous tips conspicuously broader than midribs. (Also, leaves glabrous, minutely strigose above and/or pubescent on lower midrib).

I2. Lobes of disc-corollas about $0.5 \mathrm{~mm}$ long, about I/3 total length of throat; ligules io to 30 .

I3. Pedicels very leafy, 3 to 5 times as long as heads; whole plant extremely leafy; heads scattered on branches, not in I-sided racemes; seems to prefer open field habitat ............ A. dumosus

I3. Pedicels nearly leafless, I/2 to 2 times as long as heads; plant moderately leafy; heads numerous, definitely in I-sided racemes; seems to prefer moist, partly shaded habitat ....... A. vimineus

I2. Lobes of disc-corollas I to I. $5 \mathrm{~mm}$ long, about $2 / 3$ total length of throat; ligules 7 to I4 ...... A. lateriflorus

II. Branches of stem more ascending than spreading; phyllaries loosely ascending, with herbaceous tips only slightly broader than midribs. (Also, leaves with sterile axillary fascicles).

I4. Ligules blue-violet; network of leaf veins distinct, with isodiametric areolae. (Also, leaves always entire) ........................ praealtus

I4. Ligules white; network of leaf veins obscure, with long areolae. (Also, leaves either entire or serrate) .....

A. simplex

Symphyotrichum tenuifolium (L.) G.L. Nesom

Perennial Saltmarsh Aster (Aster tenuifolius L.)

(Aster)

Java Farm. One station: Fox Creek salt marsh. Hectare 3527. Associate species: Baccharis halimifolia, Spartina cynosuroides. Higman 96. 
Java Farm. Infrequent in salt marshes and along tidal part of Muddy Creek. Associate species: Solidago sempervirens, Distichlis spicata, Cyperus strigosus, Spartina patens. Higman 210, $267,447$.

Oclemena acuminata (Michx.) Greene

Whorled Wood Aster

(A. acuminatus Michx.?)

(Aster)

Java Farm. One station: south margin of old field west of road to springhouse. Hectare 34I6. Dense Lonicera japonica, Rhus radicans, and young Liquidambar styraciflua. Also in rubbish on roof of barn. Higman 393, 356.

S. puniceum (L.) Á. Löve \& D. Löve var. puniceum

Purplestem Aster

(A. puniceus L.)

(Aster)

Java Farm. One station: side of steep bank of Fox Creek drainage. Approx. hectare 2593. Moist, shaded site. Associate species: Panicum clandestinum, Onoclea sensibilis, Leersia virginica. Higman 32I.

S. pilosum (Willd.) G.L. Nesom var. pilosum

Hairy White Oldfield Aster

(A. pilosus Willd.)

(Aster)

Java Farm. Infrequent in old fields; collected in hectares 3523 and 3585. Dense Rhus radicans, Lonicera japonica, and Rubus spp. Partial canopy of Diospyros virginiana, Liquidambar styraciflua, Quercus palustris, Prunus avium, and Viburnum prunifolium.

Also one station in rubbish on roof of barn, main building area. Accessible from tile silo. Hectare 2590. Higman 355, 426, 513.

S. novi-belgii (L.) G.L. Nesom var. novi-belgii

New York Aster

(A. novi-belgii L.)

(Aster)

Java Farm. One station: main building area. Hectare 2580. Associate species: Daucus carota, Achillea millefolium, Epilobium coloratura. Higman I97.

S. dumosum (L.) G.L. Nesom var. dumosum

Rice Button Aster (A. dumosus L.)

(Aster)

Infrequent to moderately abundant in old fields. Collected in hectare 3506, near south border of large field mostly covered by Rhus radicans. Associate species: Cirsium discolor, Eupatorium serotinum, Aster vinimeus, Poa pratensis. Higman 339. 
Java Farm. One station: roadside at main building area. Hectare 2580. Associate species: Aster novi-belgii, Achillea millefolium, Daucus carota. Higman I98.

\section{S. lateriflorum (L.) Á. Löve \& D. Löve var. lateriflorum}

Calico Aster

(A. vimineus Lam.)

(Aster)

Java Farm. Infrequent to moderately abundant along bottom of Muddy Creek valley, upstream from tidal portion: also in marshy west end of Phalaris arundinacea meadow north of main buildings (hectare 2468) and along Fox Creek drainage. All stations moist and shaded. Canopy and associate species vary. Also one station in old field, approx. hectare 2572 . This station dry, unshaded. Rhus radicans, Lonicera japonica, and Solidago altissima dense. Higman I94, 208, 329, 1042.

S. racemosum (Elliott) G.L. Nesom

(A. vimineus Lam., var. subdumosus Wieg.)
Smooth White Oldfield Aster

(Aster)

Java Farm. One station: along Fox Creek drainage. Hectare 2583, near new entrance road. Canopy of young Juglans nigra, Liquidambar styraciflua. Associate species: Elephantopus carolinianus, Panicum clandestinum, Muhlenbergia schreberi, Ambrosia trifida, Perilla frutescens, Sanicula canadensis. Higman 307.

S. lateriflorum (L.) Á. Löve \& D. Löve var. lateriflorum

Calico Aster (A. lateriflorus (L.) Britt.)

(Aster)

Java Farm. One station: along Fox Creek drainage, upstream from culvert, near north boundary of Farm. Hectare 2473. Canopy of Ulmus americana, Juglans nigra, Liquidambar styraciflua. Associate species: Dryopteris noveboracensis, Muhlenbergia schreberi. Higman 3i6.

S. praealtum (Poir.) G.L. Nesom var. praealtum

Willowleaf Aster (A. praealtus Poir.)

Java Farm. One station: freshwater part of Fox Creek drainage marsh. Hectare 3526. Associate species: Impatiens capensis, Sambucus canadensis, Salix nigra, Scirpus rubricosus. Higman 492. 
Java Farm. One station: south-facing slope above tidal portion of Muddy Creek. Source of small tributary; hectare 3426. Canopy of Liquidambar styraciflua, Fagus grandifolia, Liriodendron tulipifera, Juglans nigra. Associate species: Carex lurida, Saururus cernuus. Higman 398.

\section{Conyza, Erigeron}

I. Involucre campanulate; rays minute and in several rows, or wanting.

2. Stem glabrous except sometimes at base; leaves bristly-ciliate only at base; phyllaries with a dark purple apical spot. Expected (E. pusillus).

2. Stem spreading-hirsute throughout; leaves bristly-ciliate to tip, hispid; phyllaries without dark apical spot . . . . . . . . . . . . canadensis

I. Involucre saucer-shaped to hemispheric; rays spreading, conspicuous.

3. Cauline leaves sessile, with rounded or sub-clasping bases; disc-corollas 2.5 to $4.5 \mathrm{~mm}$ long; pappus simple.

4. Long, superficial stolons present; heads i to 7; rays about 50; disc corollas 3.5 to $4.5 \mathrm{~mm}$ long. Expected (E. pulchellus).

4. Long, superficial stolons absent; heads more numerous; rays too or more; disc-corollas 2.5 to $3.5 \mathrm{~mm}$ long. (Also, stem long-pilose below) ....... $\ldots \ldots \ldots \ldots \ldots \ldots \ldots \ldots \ldots \ldots \ldots \ldots \ldots \ldots \ldots \ldots \ldots \ldots \ldots$. philadelphicus

3. Cauline leaves tapering to base; disc corollas $\mathrm{I} .5$ to $2.5 \mathrm{~mm}$ long; pappus of disc-flowers double, with a minute outer series.

5. Leaves coarsely cleft or serrate, ovate to lanceolate, bristly-ciliate; rays longer than width of $\operatorname{disc} \ldots \ldots \ldots \ldots \ldots \ldots \ldots \ldots \ldots$. annuus

5. Leaves entire, linear-lanceolate to broadly oblanceolate, minutely strigose to glabrous; rays equal to or shorter than width of disc ..... E. strigosus

Conyza canadensis (L.) Cronquist var. canadensis

Canadian Horseweed (Erigeron canadensis L.)

(Horseweed, Fleabane, Hogweed, Butterweed)

Java Farm. Infrequent in old fields south of main building area. Hectares 2499, 3409. Associate species: Poa pratensis (dense turf), Achillea millefolium, Plantago lanceolata, Cirsium discolor, Hypericum perforatum, Asclepias syriaca, Verbascum thapsus. Higman II.

Erigeron philadelphicus L.

Philadelphia Fleabane

(Fleabane)

Ivy Neck. One station: abandoned grassy field on Scaffold Peninsula. Hectares 476I, 4772. Associate species: Trifolium pratense, T. hybricum, Oenothera fruticosa. Higman 726. 
Java Farm and Ivy Neck. Infrequent to moderately abundant in old fields. Also scattered along old entrance road between Muddy Creek culvert and main building area. Variety of associate species, including: Bromus japonicus, Agrimonia parviflora, Solidago altissima, Solanum carolinense. Often among dense Rhus radicans and Lonicera japonica. Higman 48, 750, 889, 892.

E. strigosus Muhl. ex Willd.

(E. strigosus Muhl.)

Prairie Fleabane

(White-top, Daisy Fleabane)

Java Farm. Infrequent in old fields south of main building area and south of Fox Point Road. Associate species vary, include: Solidago graminifolia, Cyperus strigosus, Lespedeza cuneata, Verbascum thapsus. Higman 49, I22.

\section{Senecioneae}

I. Heads monoecious or subdioecious, the hermaphrodite (bisexual) flowers sterile, the pistillate ones fruiting. Expected (Tussilago).

I. Heads with all or nearly all flowers fertile.

2. Cauline leaves opposite; pappus finely barbed. (Also, flowers yellow). Expected (Arnica).

2. Cauline leaves alternate; pappus capillary, soft.

3. Ray-flowers absent; corollas white.

4. Outer and inner flowers hermaphrodite; corollas 5-cleft. Expected (Cacalia).

4. Outer flowers pistillate only; corollas with 2-to 4 -toothed limb . . . . . $\ldots \ldots \ldots \ldots \ldots \ldots \ldots \ldots \ldots \ldots \ldots$ Erechtites

3. Ray-flowers usually present (as in species collected); corollas yellow or yellow with red tips $\ldots \ldots \ldots \ldots \ldots \ldots \ldots \ldots \ldots \ldots \ldots \ldots \ldots \ldots \ldots$

\section{Erechtites}

Erechtites hieraciifolius (L.) Raf. ex DC.

American Burnweed

(Erechtites hieracifolia (L.) Raf.)

(Fireweed)

Java Farm. Infrequent: two stations in old fields south of main buildings (hectare 2499) and on north side of Fox Creek drainage (hectare 3505). Also observed in Phalaris arundinacea meadow, north of main buildings (hectare 2468). Associate species vary with site. Higman 50, 537. 


\section{Packera}

(Senecio)

Packera obovata (Muhl. ex Willd.) W.A. Weber \& Á. Löve Roundleaf Ragwort (Senecio obovatus Muhl.)

(Groundsel, Squaw-weed)

Java Farm. One station: source of small tributary valley of Rhode River, on Fox Creek estuary south of pier. Hectare 3508. Canopy of Robinia pseudoacacia, Liquidambar styraciflua, and Ulmus americana. Dense ground layer of Lonicera japonica. Associate species: Verbesina occidentalis. Higman II5I.

\section{Inuleae}

I. Salt marsh to freshwater aquatic habitat; flowers pink to purple; phyllaries slightly scarious. (Also, phyllaries ciliate; plant camphor-scented) . . . Pluchea

I. Terrestrial habitat; flowers white or yellowish; phyllaries moderately to very scarious.

2. Plants dioecious, with all heads either pistillate or staminate; basal rosettes present, the cauline leaves much smaller; leaves and stem densely white-

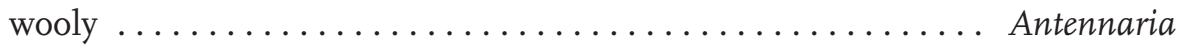

2. Plants not dioecious, the heads with outer pistillate very slender flowers and inner perfect flowers; basal rosettes absent; leaves and stem moderately

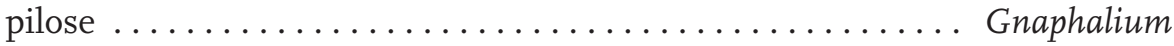

Pluchea camphorata (L.) DC.

Pluchea

Camphor Pluchea

(Stinkweed)

Java Farm and Ivy Neck. Infrequent in salt marshes near shoreward margin, and along upstream shores of estuaries. Associate species: Typha latifolia, T. angustifolia, Hibiscus palustris, Mikania scandens, Panicum clandestinum. Higman 82, II3, 2I7.

\section{Antennaria}

Antennaria plantaginifolia (L.) Richardson

(Antennaria plantaginifolia (L.) Hook.)
Woman's Tobacco

(Everlasting, Ladies'-tobacco)

Java Farm. Infrequent on eroded banks at Fox Point (hectare 3558) and along old Muddy Creek Road near old entrance gate (hectares 2472, 2482). Canopy of Quercus prinus and Q. alba at Fox Point; associate species there: Epigaea repens. Canopy at Muddy Creek Road of Fagus grandifolia, Liriodendron tulipifera, Quercus alba. Higman 568.

\section{Pseudognaphalium, Gamochaeta}

(Gnaphalium)

I. Inflorescence a corymb; phyllaries very white; bristles of pappus free to base $\ldots \ldots \ldots \ldots \ldots \ldots \ldots \ldots \ldots \ldots \ldots \ldots \ldots \ldots \ldots \ldots \ldots \ldots \ldots \ldots$ obtusifolium

I. Inflorescence a spike; phyllaries pale brown; bristles of pappus united at base G. purpureum 
Java Farm. Infrequent in old fields. Principal station in grassy area south of main building area. Hectares 2499, 3409. Associate species there: Verbascum thapsus, Solanum carolinense, Agrimonia parviflora, Cirsium discolor, Asclepias syriaca, Erigeron annuus, Erigeron strigosus, Verbascum blattaria, Apocynum cannabinum, Erechtites hieracifolia. Higman 5I, 229.

\section{Gamochaeta purpurea (L.) Cabrera}

(G. purpureum L.)

\section{Spoonleaf Purple Everlasting}

(Purple Cudweed)

Java Farm. One station: south-facing slope between grassy meadow and north boundary of Farm. Hectares 2458, 2459. Partial canopy of Pyrus malus, Liriodendron tulipifera, and Liquidambar styraciflua. Collected in small grassy area, one of several among dense Rubus spp., Lonicera japonica, and Rhus radicans. Associate species: Trifolium procumbens. Higman ıı20. 



\title{
Appendix I
}

\section{Additional Species Collected at the Chesapeake Bay Center for Field Biology during the Preparation of This Checklist}

\author{
THELYPTERIDACEAE \\ (POLYPODIACEAE)
}

Phegopteris

(Dryopteris)

Phegopteris hexagonoptera (Michx.) Fée

Broad Beechfern

(Dryopteris hexagonoptera (Michx.) Christens.)

(Thelypteris hexagonoptera (Michx.) Weatherby)

(Broad Beech-fern)

Java Farm. One station: mature forest south of old entrance road, on western side of north fork of Muddy Creek, near a small tributary. Hectare 2475. Canopy of Fagus grandifolia, Quercus spp., Liriodendron tulipifera, Carya tomentosa, etc. Higman I208.

\section{POACEAE}

(GRAMINEAE)

Eragrostis

Eragrostis pectinacea (Michx.) Nees ex Steud.

Tufted Lovegrass

(Eragrostis pectinacea (Michx.) Nees)

Java Farm. One station: parking area at headquarters, in cracked pavement. Higman 9II. 


\section{Dichanthelium}

(Panicum)

Dichanthelium villosissimum (Nash) Freckmann

Whitehair Rosette Grass

var. villosissimum

(Panicum villosissimum Nash)

Ivy Neck. One station: border of cornfield on Cheston Peninsula, adjacent young stand of mixed hardwoods. Higman 925.

\section{LILIACEAE}

Uvularia

Uvularia perfoliata L.

Perfoliate Bellwort

(Merry-bells)

Java Farm. One station: mature forest south of old entrance road, on western side of north fork of Muddy Creek. Hectare 2493, about $50 \mathrm{ft}$. southeast of grid marker. Canopy of Fagus grandifolia, Quercus spp., Liriodendron tulipifera, Carya tomentosa, etc. Higman I247.

\section{DIOSCOREACEAE}

Dioscorea

Dioscorea quaternata J.F. Gmel.

Fourleaf Yam

(Dioscorea quaternata (Walt.) J. F. Gmel.)

(Yam)

Java Farm. Infrequent in mature forest south of old entrance road, on western side of north fork of Muddy Creek. Canopy of Fagus grandifolia, Quercus spp., Liriodendron tulipifera, Carya tomentosa, etc. Higman izıо.

\section{ORCHIDACEAE}

Tipularia

Tipularia discolor (Pursh) Nutt.

Crippled Cranefly

(Cranefly Orchid)

Ivy Neck. One station in mixed hardwood forest on central Scaffold Peninsula. Canopy of Liquidambar styraciflua, Quercus spp., Liriodendron tulipifera, Carya tomentosa, etc. Higman I253. 


\section{ULMACEAE}

Ulmus

Ulmus procera Salisb.

English Elm

Java Farm. One station: abandoned field on shore of Fox Creek estuary, near pier. Approx. hectare 36ro. Associate species: scattered Ulmus rubra, Ulmus americana, Acer negundo, Liquidambar styraciflua. Dense ground cover of Rhus radicans and Lonicera japonica.

\section{POLYGONACEAE}

Polygonum

Polygonum scandens L. var. cristatum

Climbing False Buckwheat

(Engelm. \& A. Gray) Gleason

(Polygonum cristatum Engelm. \& Gray)

(Cornbind)

Java Farm. One station: beside old entrance road at culvert of small stream, on east side of north fork of Muddy Creek. Hectare 2497. Canopy of Platanus occidentalis. Associate species: Galium aparine, Commelina communis, Duchesnea indica. Higman 1207.

\section{BERBERIDACEAE}

Berberis

Berberis thunbergii DC.

Japanese Barberry

Java Farm. One station: bank of north fork of Muddy Creek, north of old entrance road. Canopy of Platanus occidentalis. Higman I250.

\section{BRASSICACEAE \\ (CRUCIFERAE) \\ Capsella}

Capsella bursa-pastoris (L.) Medik.

Shepherd's Purse (Capsella bursa-pastoris (L.) Medic.)

Java Farm. One station: recently cleared area at headquarters, near concrete silo. Higman 1256. 


\section{LAMIACEAE}

(LABIATAE)

Agastache

Agastache nepetoides (L.) Kuntze

(Giant Hyssop)

Yellow Giant Hyssop

Java Farm. One station: north-facing slope overlooking small stream which drains into Fox Creek estuary. Hectare 3518. Canopy of Robinia pseudoacacia, Liquidambar styraciflua, and Ulmus americana. Higman Io40.

Lycopus

Lycopus europaeus L.

Gypsywort

(Bugleweed)

Ivy Neck. One station: margin of small cut-off pond on Cheston Peninsula, below Sand Point. Hectare 4870. Associate species: Rumex verticillatus, Echinochloa walteri. Higman 1232 .

\section{SOLANACEAE}

Solanum

Solanum americanum Mill.

American Black Nightshade

(not in Britton \& Brown)

(Nightshade)

Ivy Neck. One station: crest of sandbar of small cut-off pond on Cheston Peninsula, below Sand Point. Hectare 4870. Associate species: Spartina alterniflora, Tripsacum dactyloides. Higman 1222. 


\section{Appendix II}

\section{Additional Annotations for Species in This Checklist}

Osmunda regalis $\mathrm{L}$.

Royal Fern

Java Farm. One station in Muddy Creek valley near upstream limit of salt marsh. Hectare 2493. Canopy of Fagus grandifolia.

\section{PINACEAE}

Pinus virginiana Mill.

Pinus

Java Farm. Infrequent at northwest border of Hog Island salt marsh. Hectare 357I. Associate species: Quercus falcata, Q. phellos.

\section{POACEAE \\ (GRAMINEAE) \\ Echinochloa}

Echinochloa walteri (Pursh) A. Heller

Coast Cockspur Grass

(Echinochloa walteri (Pursh) Nash)

Ivy Neck. Moderately abundant in marshy inland part of Sand Point, and on inland shores of cut-off ponds on Cheston Peninsula below Sand Point. Hectares 4860 , 4870, 5800. Associate species: Panicum virgatum, Scirpus spp. 


\title{
COMMELINACEAE
}

Commelina

Commelina communis L.

Asiatic Dayflower

Java Farm. One station along old entrance road, near culvert of small tributary to Muddy Creek. Hectare 2497. Canopy of Platanus occidentalis.

\section{DIOSCOREACEAE}

Dioscorea

Dioscorea villosa L.

Java Farm. Infrequent in mature forest south of old entrance road. Approx. hectare 2485. Canopy of Fagus grandifolia, Liriodendron tulipifera, Quercus spp., Carya tomentosa.

\section{CARYOPHYLLACEAE}

Silene

Silene antirrhina L.

Sleepy Silene

Java Farm. Principal station near southern border of main building area, near brick wall (now mostly destroyed). Very infrequent in old fields.

\author{
ROSACEAE \\ Amelanchier \\ Amelanchier arborea (Michx. f.) Fernald \\ Common Serviceberry \\ (Amelanchier arborea (Michx. f.) Fern.) \\ Java Farm. Infrequent on Fox Point. Hectare 3558. Canopy of Quercus prinus.
}

ONAGRACEAE

Ludwigia

Ludwigia palustris (L.) Elliott

Marsh Seedbox

(Ludwigia palustris (L.) Ell.)

Ivy Neck. Muddy shore of small cut-off pond on Cheston Peninsula south of Sand Point. Hectare 4870. Associate species: Galinsoga ciliata, Rumex verticillatus. 


\section{RUBIACEAE}

Mitchella repens L.

Mitchella

Ivy Neck. Infrequent in forest of central Scaffold Peninsula. Canopy of Quercus alba, Liriodendron tulipifera, etc.

Triodanis perfoliata (L.) Nieuwl.

CAMPANULACEAE

Triodanis

(Specularia)

(Specularia perfoliata (L.) A. DC.)

Ivy Neck. Infrequent at borders of cultivated fields.

Clasping Venus' Looking-glass

ASTERACEAE

(COMPOSITAE)

Pyrrhopappus

Pyrrhopappus carolinianus (Walter) DC.

Carolina Desert-chicory

(Pyrrhopappus carolinianus (Walt.) DC.)

Ivy Neck. Infrequent in pasture between forks of Cheston Creek drainage.

Galinsoga quadriradiata Cav.

Galinsoga

(Galinsoga ciliata (Raf.) Blake)

Shaggy Soldier

Ivy Neck. Infrequent on margin of marshy area in interior of Sand Point, and at borders of cut-off ponds on Cheston Peninsula below Sand Point. Hectares 4860 , 4870,5800 .

Eupatorium perfoliatum L.

Eupatorium

Ivy Neck. One station along Cheston Creek drainage. Hectare 4743. Canopy of Diospyros virginiana, Robinia pseudoacacia.

Verbesina

Verbesina occidentalis L.

Yellow Crownbeard

Ivy Neck. Infrequent on inland shore of small cut-off pond on Cheston Peninsula below Sand Point. Hectare 4870. 



\section{Appendix III}

\section{Common Names of Plants at the Chesapeake Bay Center, Local to That Vicinity}

(Additional local names are being sought.)

Abutilon theophrasti Medik. . Velvetleaf

(Abutilon theophrasti) (Hemp)

Cyperus strigosus $\mathbf{L}$. Strawcolored Flatsedge

(Cyperus strigosus) (Nut Grass)

Solanum carolinense $\mathrm{L}$ Carolina horsenettle (Solanum carolinense) (Poor Man's Potato)

Robinia pseudoacacia L. Black Locust (Robinia pseudoacacia) (Yellow Locust, Shipmast Locust)

Celtis occidentalis $\mathrm{L}$. Common Hackberry (Celtis occidentalis) (Sugarberry)

Quercus falcata Michx. Southern Red Oak (Quercus falcate) (Red Oak) 



\section{Appendix IV}

\section{New Plant Species Found after Original \\ Publication of Higman's Checklist}

\begin{tabular}{|c|c|c|}
\hline$\underline{\text { DIVISION AND FAMILY }}$ & SCIENTIFIC NAME & COMMON NAME \\
\hline \multicolumn{3}{|l|}{ Lycopodiophyta } \\
\hline Lycopodiaceae & Huperzia lucidula (Michx.) Trevis. & Shining Clubmoss \\
\hline \multicolumn{3}{|l|}{ Pteridophytes } \\
\hline Dennstaedtiaceae & Dennstaedtia punctilobula (Michx.) T. Moore & $\begin{array}{l}\text { Eastern Hayscented } \\
\text { Fern }\end{array}$ \\
\hline Blechnaceae & Woodwardia areolata (L.) T. Moore & Netted Chainfern \\
\hline \multicolumn{3}{|l|}{ Monocots } \\
\hline Potamogetonaceae & Stuckenia pectinata (L.) Börner & Sago Pondweed \\
\hline Ruppiaceae & Ruppia maritima L. & Widgeongrass \\
\hline \multirow[t]{8}{*}{ Poaceae } & $\begin{array}{l}\text { Dichanthelium boscii (Poir.) Gould \& } \\
\text { C.A. Clark }\end{array}$ & Bosc's Panicgrass \\
\hline & Dichanthelium laxiflorum (Lam.) Gould & $\begin{array}{l}\text { Openflower Rosette } \\
\text { Grass }\end{array}$ \\
\hline & Dichanthelium scoparium (Lam.) Gould & Velvet Panicum \\
\hline & Digitaria sanguinalis (L.) Scop. & Hairy Crabgrass \\
\hline & Festuca subverticillata (Pers.) Alexeev & Nodding Fescue \\
\hline & Setaria italica (L.) P. Beauv. & Foxtail Millet \\
\hline & Setaria parviflora (Poir.) Kerguélen & Marsh Bristlegrass \\
\hline & Sphenopholis obtusata (Michx.) Scribn. & Prairie Wedgescale \\
\hline \multirow[t]{3}{*}{ Cyperaceae } & Carex gracillima Schwein. & Graceful Sedge \\
\hline & Carex grisea Wahlenb. & $\begin{array}{l}\text { Inflated Narrow-Leaf } \\
\text { Sedge }\end{array}$ \\
\hline & Carex hystericina Muhl. ex Willd. & $\begin{array}{l}\text { Bottlebrush Sedge } \\
\text { (continued) }\end{array}$ \\
\hline
\end{tabular}




\begin{tabular}{|c|c|c|}
\hline DIVISION AND FAMILY & SCIENTIFIC NAME & COMMON NAME \\
\hline \multicolumn{3}{|c|}{ Cyperaceae (continued) } \\
\hline & Carex laevivaginata (Kük.) Mack. & $\begin{array}{l}\text { Smoothsheath } \\
\text { Sedge }\end{array}$ \\
\hline & Carex lupulina Muhl. ex Willd. & Hop Sedge \\
\hline & Carex striata Michx. & Walter'S Sedge \\
\hline \multirow[t]{2}{*}{ Araceae } & Orontium aquaticum $\mathrm{L}$. & Goldenclub \\
\hline & Peltandra virginica (L.) Schott & Green Arrow Arum \\
\hline Juncaceae & Juncus roemerianus Scheele & Needlegrass Rush \\
\hline \multirow[t]{4}{*}{ Liliaceae } & Erythronium americanum Ker Gawl. & Dogtooth Violet \\
\hline & Medeola virginiana L. & Indian Cucumber \\
\hline & Polygonatum biflorum (Walter) Elliott & $\begin{array}{l}\text { Smooth Solomon's } \\
\text { Seal }\end{array}$ \\
\hline & Uvularia sessilifolia L. & Sessileleaf Bellwort \\
\hline Smilacaceae & Smilax tamnoides L. & Bristly Greenbrier \\
\hline Iridaceae & Iris germanica L. & German Iris \\
\hline \multirow[t]{4}{*}{ Orchidaceae } & Corallorhiza odontorhiza (Willd.) Poir. & Autumn Coralroot \\
\hline & Aplectrum hyemale (Muhl. ex Willd.) Torr. & Adam And Eve \\
\hline & Liparis liliifolia (L.) Rich. ex Ker Gawl. & $\begin{array}{l}\text { Brown Widelip } \\
\text { Orchid }\end{array}$ \\
\hline & Platanthera lacera (Michx.) G. Don & $\begin{array}{l}\text { Green Fringed } \\
\text { Orchid }\end{array}$ \\
\hline \multicolumn{3}{|l|}{ Dicots } \\
\hline Polygonaceae & Polygonum perfoliatum L. & Asiatic Tearthumb \\
\hline Juglandaceae & Carya ovalis (Wangenh.) Sarg. & Red Hickory \\
\hline \multirow[t]{4}{*}{ Fagaceae } & Castanea mollissima Blume & Chinese Chestnut \\
\hline & Quercus ilicifolia Wangenh. & Bear Oak \\
\hline & Quercus michauxii Nutt. & $\begin{array}{l}\text { Swamp Chestnut } \\
\text { Oak }\end{array}$ \\
\hline & Quercus rubra L. & Northern Red Oak \\
\hline Urticaceae & Pilea pumila (L.) A. Gray & Canadian Clearweed \\
\hline Portulacaceae & Portulaca oleracea L. & Little Hogweed \\
\hline \multirow[t]{4}{*}{ Caryophyllaceae } & Cerastium arvense $\mathrm{L}$. & Field Chickweed \\
\hline & Saponaria officinalis L. & Bouncingbet \\
\hline & Scleranthus annuus L. & German Knotgrass \\
\hline & Stellaria graminea L. & Grass-Like Starwort \\
\hline
\end{tabular}


DIVISION AND FAMILY

Dicots (continued)

Ranunculaceae

Brassicaceae

Capparaceae

Myricaceae

Hydrangaceae

Elaeagnaceae

Rosaceae

Fabaceae

Oxalidaceae

Euphorbiaceae

Aquifoliaceae

Celastraceae

Malvaceae
Actaea racemosa $\mathrm{L}$.

Aquilegia canadensis L.

Ranunculus acris L.

Ranunculus ficaria L.

Ranunculus recurvatus Poir.

Ranunculus sardous Crantz

Alliaria petiolata

Brassica rapa L.

Cleome hassleriana Chod.

Morella pensylvanica (Mirb.) Kartesz

Hydrangea arborescens L.

Elaeagnus umbellata Thunb.

Agrimonia pubescens Wallr.

Geum vernum (Raf.) Torr. \& A. Gray

Photinia pyrifolia (Lam.) K.R. Robertson

\& Phipps

Prunus americana Marshall

Rubus allegheniensis Porter

Albizia julibrissin Durazz.

Cytisus scoparius (L.) Link

Desmodium nudiflorum (L.) DC.

Gleditsia triacanthos L.

Kummerowia striata (Thunb.) Schindl.

Lespedeza capitata Michx.

Vicia grandiflora Scop.

Oxalis violacea $\mathrm{L}$.

Chamaesyce polygonifolia (L.) Small

Euphorbia cyparissias L.

llex verticillata (L.) A. Gray

Celastrus orbiculatus Thunb.

Malva neglecta Wallr.
Black Baneberry

Red Columbine

Tall Buttercup

Fig Buttercup

Blisterwort

Hairy Buttercup

Garlic Mustard

Field Mustard

Pink Queen

Northern Bayberry

Wild Hydrangea

Autumn Olive

Soft Agrimony

Spring Avens

Red Chokeberry

American Plum

Allegheny Blackberry

Silktree

Scotch Broom

Nakedflower

Ticktrefoil

Honeylocust

Japanese Clover

Roundhead

Lespedeza

Large Yellow Vetch

Violet Woodsorrel

Seaside Sandmat

Cypress Spurge

Common

Winterberry

Oriental Bittersweet

Common Mallow

(continued) 


\begin{tabular}{|c|c|c|}
\hline DIVISION AND FAMILY & SCIENTIFIC NAME & COMMON NAME \\
\hline \multicolumn{3}{|l|}{ Dicots (continued) } \\
\hline \multirow[t]{4}{*}{ Violaceae } & Viola blanda Willd. & Sweet White Violet \\
\hline & Viola cucullata Aiton & Marsh Blue Violet \\
\hline & Viola sagittata Aiton & Arrowleaf Violet \\
\hline & Viola triloba Schwein. & Three-Lobe Violet \\
\hline Lythraceae & Lythrum salicaria L. & Purple Loosestrife \\
\hline \multirow[t]{4}{*}{ Apiaceae } & Cicuta maculata L. & $\begin{array}{l}\text { Spotted Water } \\
\text { Hemlock }\end{array}$ \\
\hline & Osmorhiza claytonii (Michx.) C.B. Clarke & Clayton's Sweetroot \\
\hline & Osmorhiza longistylis (Torr.) DC. & Longstyle Sweetroot \\
\hline & Zizia aptera (A. Gray) Fernald & Meadow Zizia \\
\hline \multirow[t]{2}{*}{ Ericaceae } & Rhododendron atlanticum (Ashe) Rehder & Dwarf Azalea \\
\hline & Vaccinium fuscatum Aiton & $\begin{array}{l}\text { Black Highbush } \\
\text { Blueberry }\end{array}$ \\
\hline Primulaceae & Lysimachia quadrifolia L. & $\begin{array}{l}\text { Whorled Yellow } \\
\text { Loosestrife }\end{array}$ \\
\hline Viscaceae & $\begin{array}{l}\text { Phoradendron leucarpum (Raf.) Reveal } \\
\text { \& M.C. Johnst. }\end{array}$ & Oak Mistletoe \\
\hline \multirow[t]{2}{*}{ Oleaceae } & Forsythia suspensa (Thunb.) Vahl & Weeping Forsythia \\
\hline & Fraxinus pennsylvanica Marshall & Green Ash \\
\hline Apocynaceae & Vinca minor $\mathrm{L}$. & Common Periwinkle \\
\hline \multirow[t]{3}{*}{ Asclepiadaceae } & Asclepias amplexicaulis Sm. & Clasping Milkweed \\
\hline & Asclepias tuberosa L. & Butterfly Milkweed \\
\hline & Matelea carolinensis (Jacq.) Woodson & $\begin{array}{l}\text { Maroon Carolina } \\
\text { Milkvine }\end{array}$ \\
\hline Convolvulaceae & Ipomoea coccinea L. & Redstar \\
\hline Boraginaceae & Myosotis macrosperma Engelm. & $\begin{array}{l}\text { Largeseed } \\
\quad \text { Forget-Me-Not }\end{array}$ \\
\hline \multirow[t]{4}{*}{ Lamiaceae } & Leonurus cardiaca L. & $\begin{array}{l}\text { Common } \\
\text { Motherwort }\end{array}$ \\
\hline & $\begin{array}{l}\text { Lycopus americanus Muhl. ex } \\
\text { W.P.C. Barton }\end{array}$ & $\begin{array}{c}\text { American Water } \\
\text { Horehound }\end{array}$ \\
\hline & Monarda punctata L. & Spotted Beebalm \\
\hline & Salvia urticifolia L. & Nettleleaf Sage \\
\hline Solanaceae & Physalis heterophylla Nees & $\begin{array}{l}\text { Clammy } \\
\text { Groundcherry }\end{array}$ \\
\hline
\end{tabular}




\begin{tabular}{|c|c|c|}
\hline DIVISION AND FAMILY & SCIENTIFIC NAME & COMMON NAME \\
\hline \multicolumn{3}{|l|}{ Dicots (continued) } \\
\hline \multirow[t]{6}{*}{ Scrophulariaceae } & Agalinis purpurea (L.) Pennell & $\begin{array}{c}\text { Purple False } \\
\text { Foxglove }\end{array}$ \\
\hline & Chelone glabra L. & White Turtlehead \\
\hline & Mimulus alatus Aiton & $\begin{array}{l}\text { Sharpwing } \\
\text { Monkeyflower }\end{array}$ \\
\hline & Penstemon digitalis Nutt. ex Sims & $\begin{array}{l}\text { Foxglove } \\
\text { Beardtongue }\end{array}$ \\
\hline & Veronica officinalis $\mathrm{L}$. & $\begin{array}{l}\text { Common } \\
\text { Gypsyweed }\end{array}$ \\
\hline & Veronica serpyllifolia L. & $\begin{array}{l}\text { Thymeleaf } \\
\text { Speedwell }\end{array}$ \\
\hline Caprifoliaceae & Lonicera maackii (Rupr.) Herder & Amur Honeysuckle \\
\hline Callitrichaceae & Callitriche heterophylla Pursh & $\begin{array}{l}\text { Twoheaded } \\
\text { Water-Starwort }\end{array}$ \\
\hline \multirow[t]{16}{*}{ Asteraceae } & Carduus nutans L. & $\begin{array}{l}\text { Nodding Plumeless } \\
\text { Thistle }\end{array}$ \\
\hline & Centaurea nigrescens Willd. & Tyrol Knapweed \\
\hline & Centaurea stoebe L. & Spotted Knapweed \\
\hline & Chrysopsis mariana (L.) Elliott & $\begin{array}{l}\text { Maryland } \\
\text { Goldenaster }\end{array}$ \\
\hline & Cosmos bipinnatus Cav. & Garden Cosmos \\
\hline & Eurybia divaricata (L.) G.L. Nesom & White Wood Aster \\
\hline & Eutrochium maculatum (L.) E.E. Lamont & $\begin{array}{l}\text { Spotted Joe Pye } \\
\text { Weed }\end{array}$ \\
\hline & Helianthus tuberosus L. & $\begin{array}{l}\text { Jerusalem } \\
\text { Artichoke }\end{array}$ \\
\hline & Hieracium caespitosum Dumort. & Meadow Hawkweed \\
\hline & Krigia virginica (L.) Willd. & $\begin{array}{l}\text { Virginia } \\
\text { Dwarfdandelion }\end{array}$ \\
\hline & Oligoneuron rigidum (L.) Small & Stiff Goldenrod \\
\hline & Pluchea odorata (L.) Cass. & Sweetscent \\
\hline & Senecio vulgaris $\mathrm{L}$. & $\begin{array}{l}\text { Old-Man-In-The- } \\
\text { Spring }\end{array}$ \\
\hline & Solidago canadensis L. & Canada Goldenrod \\
\hline & Tussilago farfara L. & Coltsfoot \\
\hline & Youngia japonica (L.) DC. & $\begin{array}{l}\text { Oriental False } \\
\text { Hawksbeard }\end{array}$ \\
\hline
\end{tabular}


In May 1968, botanist Daniel Higman published An Ecologically Annotated Checklist of the Vascular Flora at the Chesapeake Bay Center for Field Biology, with Keys through the Smithsonian Institution's Office of Ecology. Henceforth referred to as The Flora, it was based on Higman's exploration of plant species found on three tracts of Smithsonian-owned land near the Chesapeake Bay in Maryland known as Java Farm, Corn Island, and Ivy Neck, which together represent a broad spectrum of ecological systems.

As part of the fiftieth anniversary celebration of the Smithsonian Environmental Research Center (SERC), this first-known SERC publication has been updated by editors Dennis Whigham, Geoffrey Parker, and Olav Oftedal and is reprinted with support from the Smithsonian's Atherton Seidell Grant Program for the Dissemination of Previously Published Scientific Research.

\section{Smithsonian Environmental Research Center}

www.serc.si.edu

\section{Smithsonian Institution} Scholarly Press

www.scholarlypress.si.edu

ISBN (online): 978-I-935623-99-I ISBN (print): 978-I-935623-98-4 